\title{
Proton-Sensitive Free Radical Dimer Evolution Is a Critical Control Point for the Synthesis of $\Delta^{2,2^{\prime}}$-Bibenzothiazines.
}

Luca Valgimigli, ${ }^{\dagger *}$ Maria Laura Alfieri, ${ }^{\ddagger}$ Riccardo Amorati, ${ }^{\dagger}$ Andrea Baschieri, ${ }^{\dagger}$ Orlando Crescenzi, ${ }^{\star}$ Alessandra Napolitano, ${ }^{*}$ and Marco d'Ischia

†Department of Chemistry “Giacomo Ciamician”, University of Bologna, I-40126 Bologna, Italy

${ }^{\ddagger}$ Department of Chemical Sciences, University of Naples Federico II, I-80126, Naples, Italy

\section{Supporting Information}

\begin{tabular}{|c|c|}
\hline Content & Page \\
\hline Figure S1. UV-vis analysis of the oxidation of dimer $\mathbf{2}$ with peroxides and hydroperoxides. & S2 \\
\hline Figure S2. Development of the chromophore of dimers $\mathbf{3}$ at $598 \mathrm{~nm}$ from $\mathbf{2}$. & S3 \\
\hline Figure S3. EPR spectrum of a solution of 1 in acidic $\mathrm{MeOH}$ and simulated spectra. & S4 \\
\hline Figure S4. Control experiment of oxygen evolution from $\mathrm{H}_{2} \mathrm{O}_{2}$ in $\mathrm{MeOH} 3 \mathrm{M} \mathrm{H}_{2} \mathrm{SO}_{4}$ at $303 \mathrm{~K}$. & S5 \\
\hline Figure S5. Oxygen uptake measured by incubating $6.5 \mu$ moles of monomer $1 \mathrm{in} 4 \mathrm{~mL} \mathrm{MeOH} 3 \mathrm{M} \mathrm{H}_{2} \mathrm{SO}_{4}$. & S6 \\
\hline Figure S6. UV-vis absorption spectra of substituted anilines in 3:1 methanol/36\% $\mathrm{HCl}$. & S7 \\
\hline $\begin{array}{l}\text { Figure S7. Computed free energy diagram for reagents, products and putative intermediates in the reaction pathway leading from } \\
\mathbf{2} \text { to } \mathbf{3} \text { (reaction path a: see text), under different } \mathrm{pH} \text { conditions. }\end{array}$ & S9 \\
\hline $\begin{array}{l}\text { Figure S8. Computed free energy diagram for reagents, products and putative intermediates in the reaction pathway leading from } \\
\mathbf{2} \text { to } \mathbf{3} \text { (reaction path b: see text) under different } \mathrm{pH} \text { conditions. }\end{array}$ & S10 \\
\hline Table S1. EPR parameters computed for the predominant forms of $\mathrm{N}$-monoprotonated $\mathrm{C}$-radicals from single-bond dimers in $\mathrm{MeOH}$. & S11 \\
\hline Table S2. EPR parameters computed for the predominant forms of $N, N^{\prime}$-diprotonated $C$-radicals from single-bond dimers in $M e O H$. & S15 \\
\hline Table S3. Calculations on neutral forms in vacuo. & S19 \\
\hline Table S4. Calculations on neutral forms in methanol. & S57 \\
\hline Table S5. Calculations on monoprotonated forms in methanol. & S95 \\
\hline Table S6. Calculations on diprotonated forms in methanol. & S157 \\
\hline Table S7. $\mathrm{p} K_{\mathrm{a}}$ values computed in methanol at different theory levels for all bibenzothiazine derivatives examined. & S191 \\
\hline $\begin{array}{l}\text { Table S8. } p K_{\mathrm{a}} \text { values computed in methanol at different theory levels for some reference nitrogen bases and corresponding } \\
\text { experimental values. }\end{array}$ & S192 \\
\hline
\end{tabular}




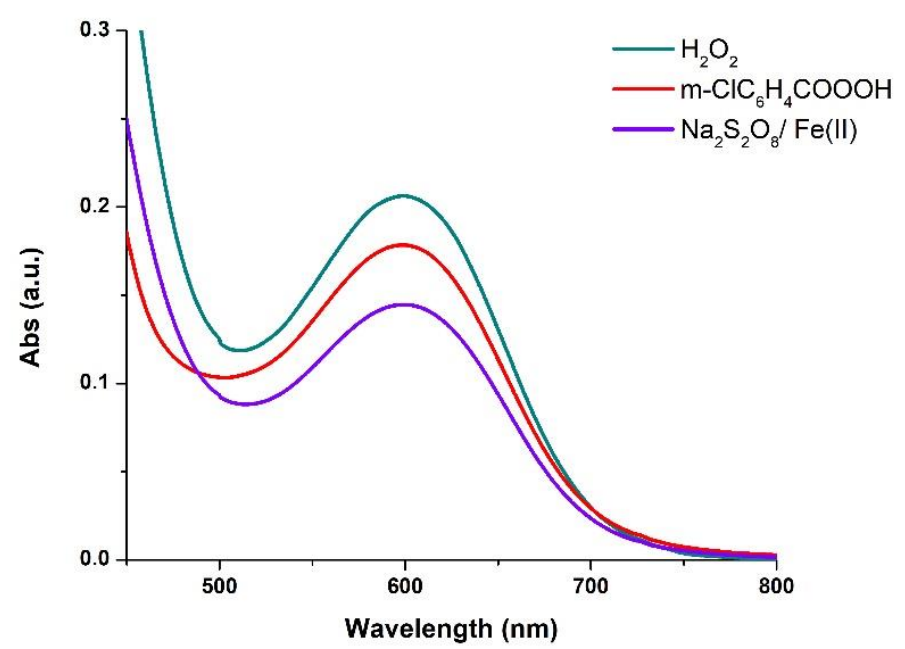

Figure S1. UV-vis analysis of the oxidation of dimer 2 at $50 \mu \mathrm{M}$ in $\mathrm{MeOH}: 36 \% \mathrm{HCl}$ 3:1 with hydrogen peroxide (0.4 equiv.), m-chloroperbenzoic acid ( $\mathrm{m}-\mathrm{ClC}_{6} \mathrm{H}_{4} \mathrm{COOOH}$ ) (0.8 equiv.) and sodium persulfate/Fe(II) (1.5 equiv) at 5 min reaction time. 


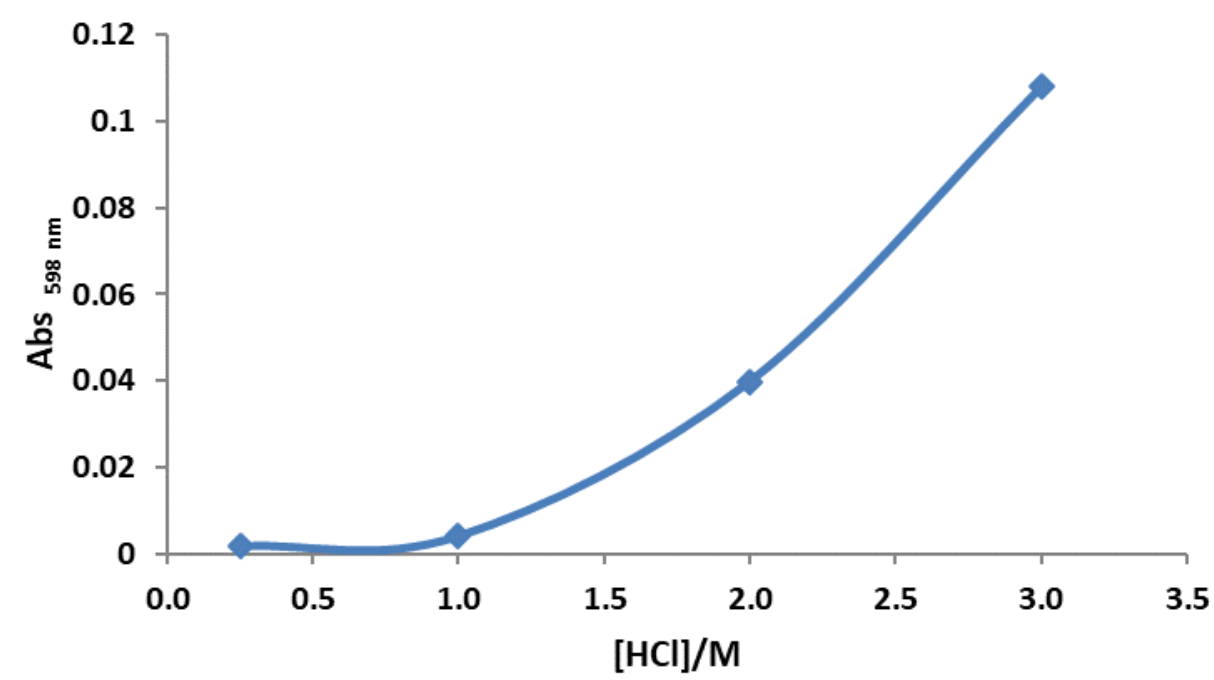

Figure S2. Development of the chromophore of dimers 3 at $598 \mathrm{~nm}$ from $2(50 \mu \mathrm{M})$ in methanol / $\mathrm{HCl}$ 3:1 at different final $\mathrm{HCl}$ concentrations in the presence of $\mathrm{H}_{2} \mathrm{O}_{2}(500 \mu \mathrm{M})$ at $30 \mathrm{~min}$. 


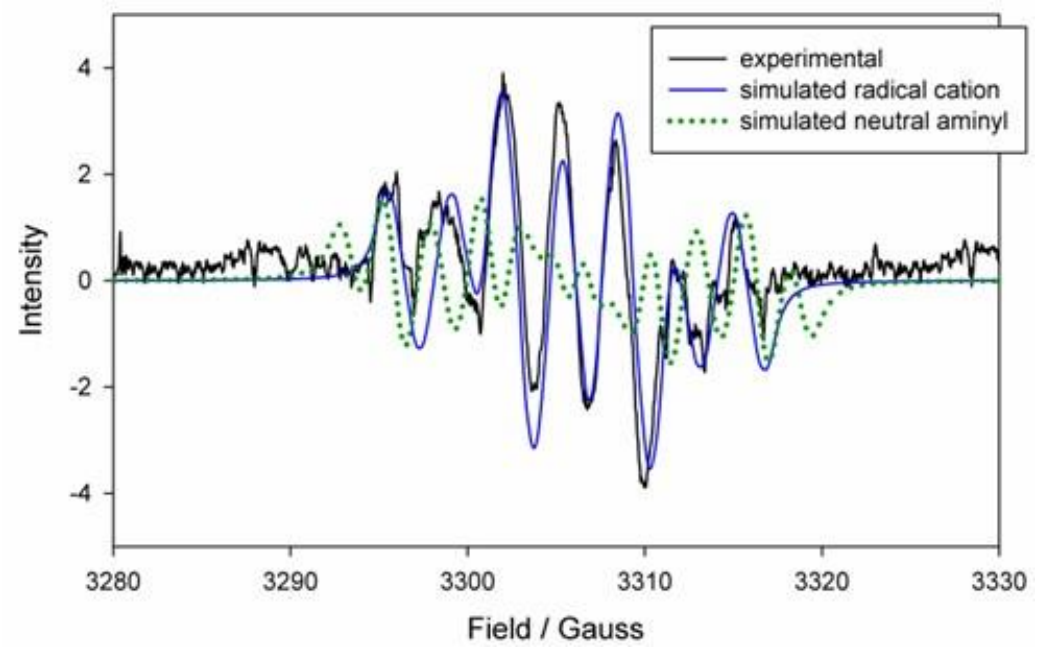

Figure S3. EPR spectrum of a solution of 1 in acidic $\mathrm{MeOH}$ and simulated spectra of the neutral and protonated free radical $\mathbf{1}$. 


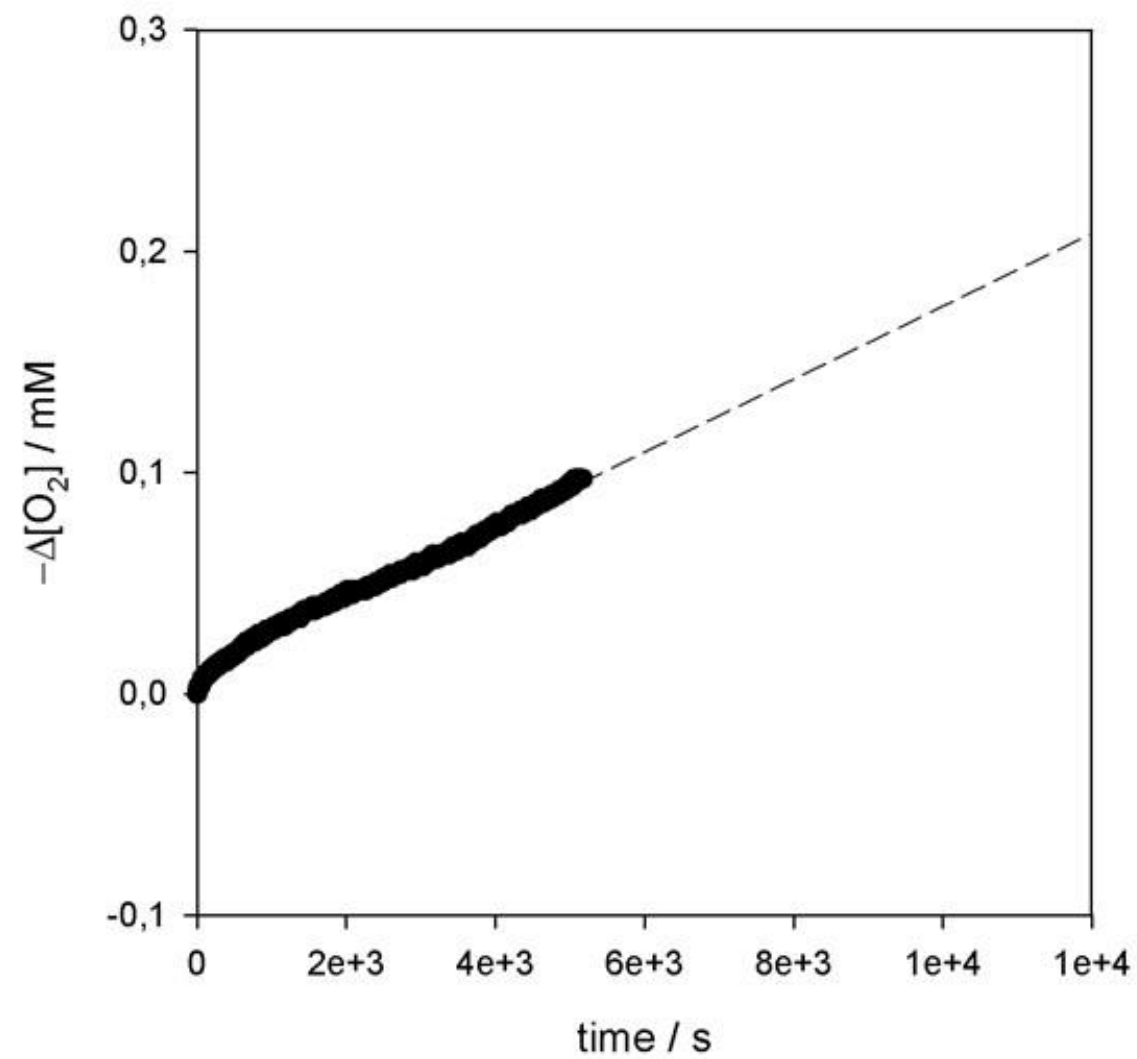

Figure S4. Oxygen evolution measured by adding $10 \mu$ moles of $\mathrm{H}_{2} \mathrm{O}_{2}$ in $4 \mathrm{~mL} \mathrm{MeOH} 3 \mathrm{M} \mathrm{H}_{2} \mathrm{SO}_{4}$ at $303 \mathrm{~K}$ in the absence of monomer 1 or dimer 2. 


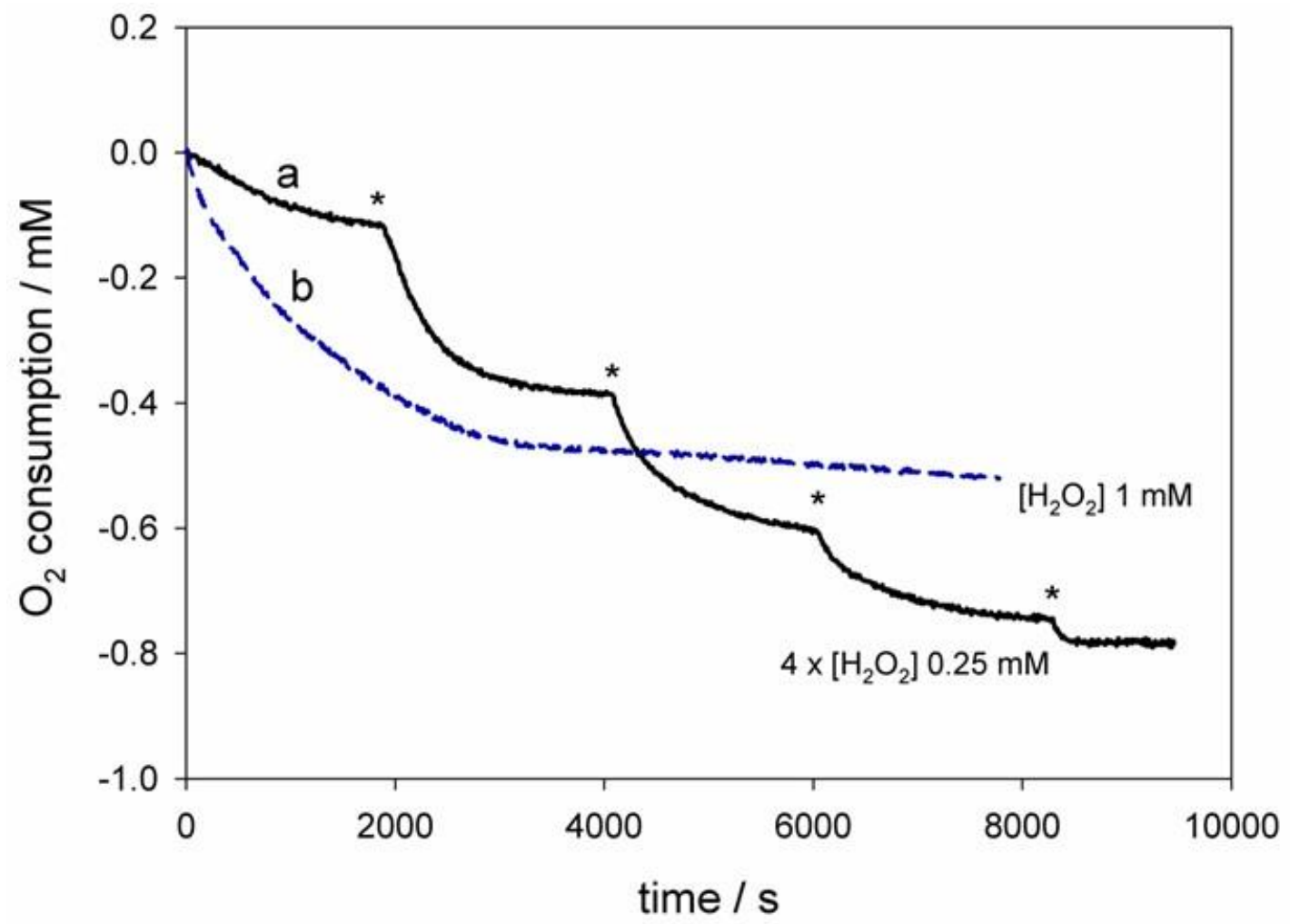

Figure 55. Oxygen uptake measured by incubating 6.5 moles of monomer 1 in $4 \mathrm{~mL} \mathrm{MeOH} 3 \mathrm{M} \mathrm{H}_{2} \mathrm{SO}_{4}$ (final conc. $1.6 \mathrm{mM}$ ) at $303 \mathrm{~K}$ with (a) addition of aliquots of $1 \mu$ mole $\mathrm{H}_{2} \mathrm{O}_{2}$ to the system at time points indicated by an asterisk, or (b) by single addition of 4 molar equivalents of $\mathrm{H}_{2} \mathrm{O}_{2}(1 \mathrm{mM})$. 


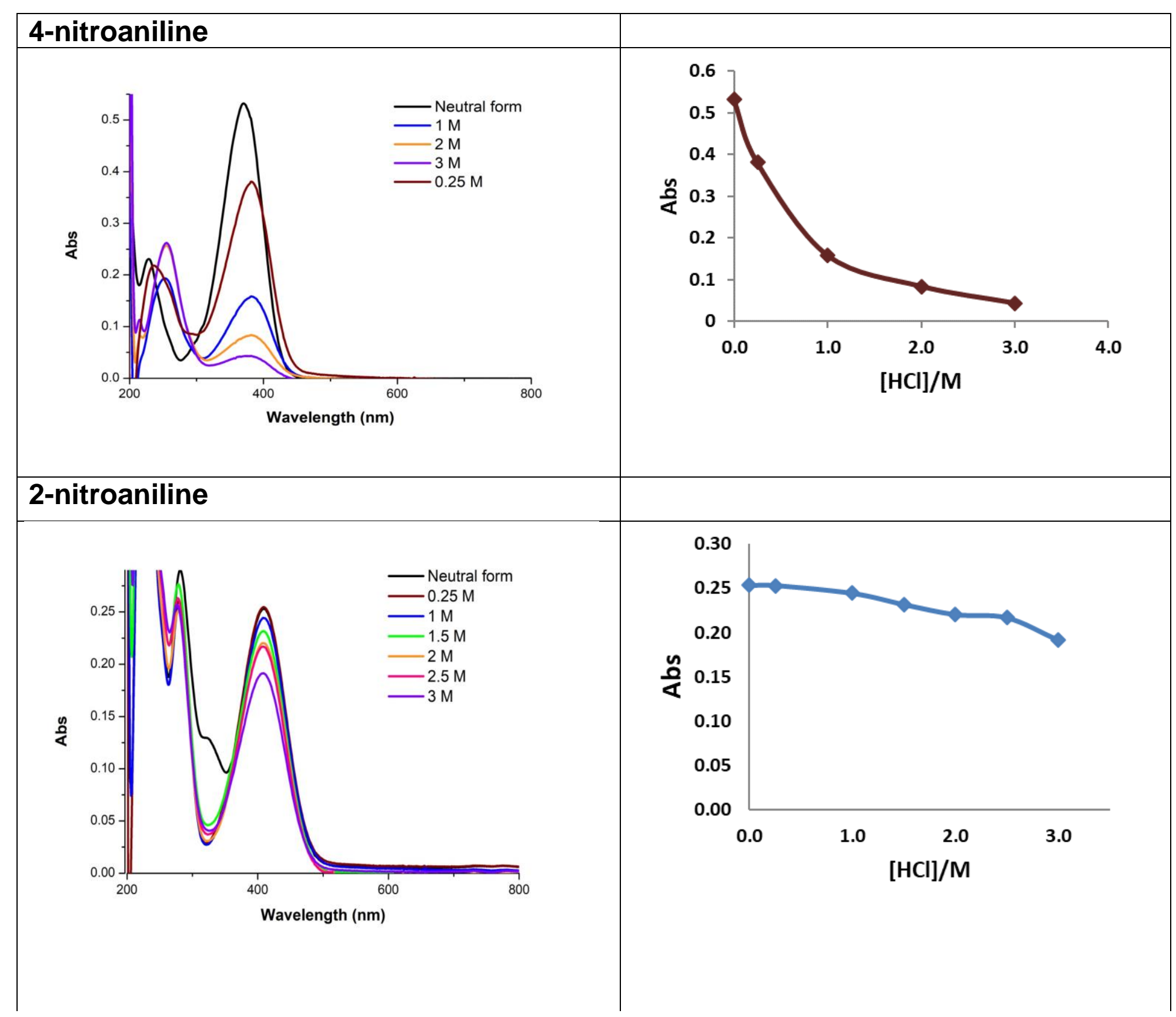




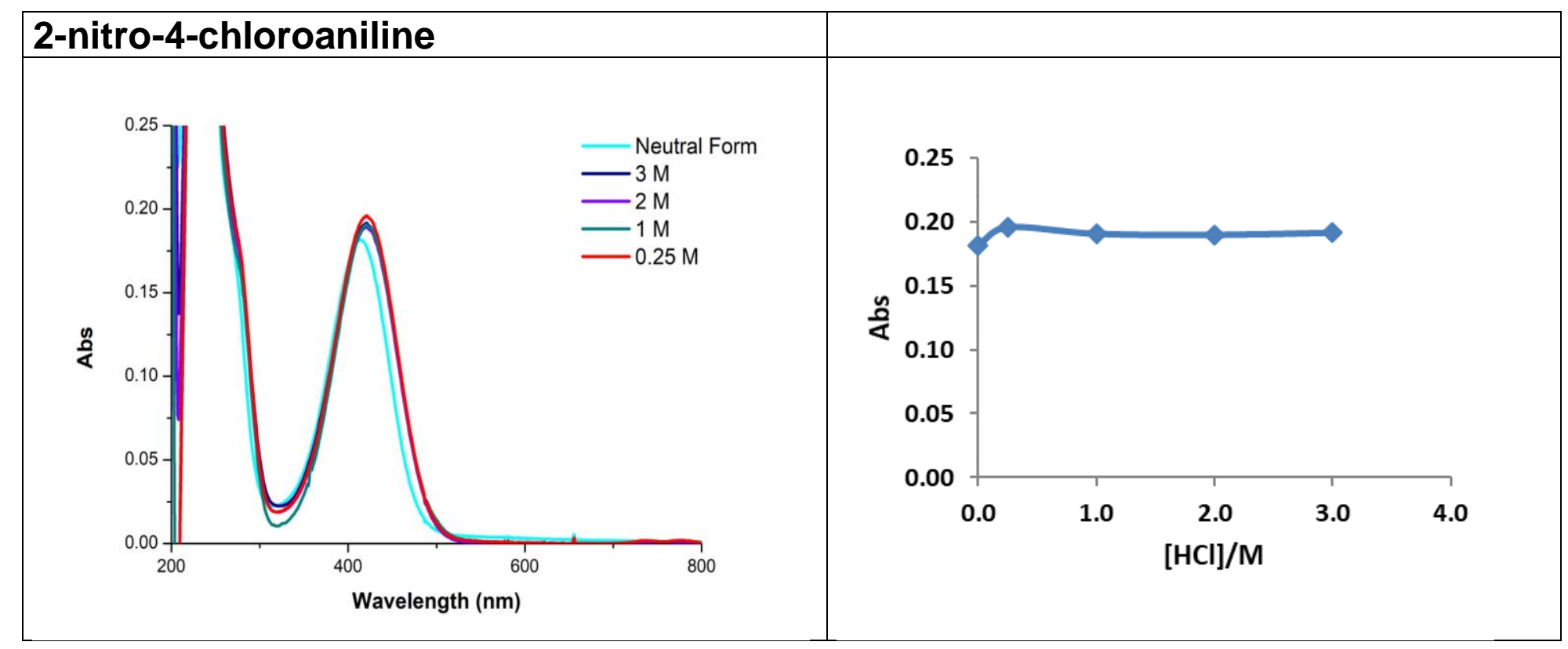

Figure S6. Left: Absorption spectra of substituted anilines in 3:1 methanol/HCl at the concentrations shown. Right: Absorbance at maximum vs $\mathrm{HCl}$ concentration. Choice of anilines was based on their $\mathrm{p} K_{\mathrm{a}} \mathrm{s}$ in methanol as reported by Rived, F.; Roses, M.; Bosch, E. Anal. Chim. Acta 1998, 374, 309324. 


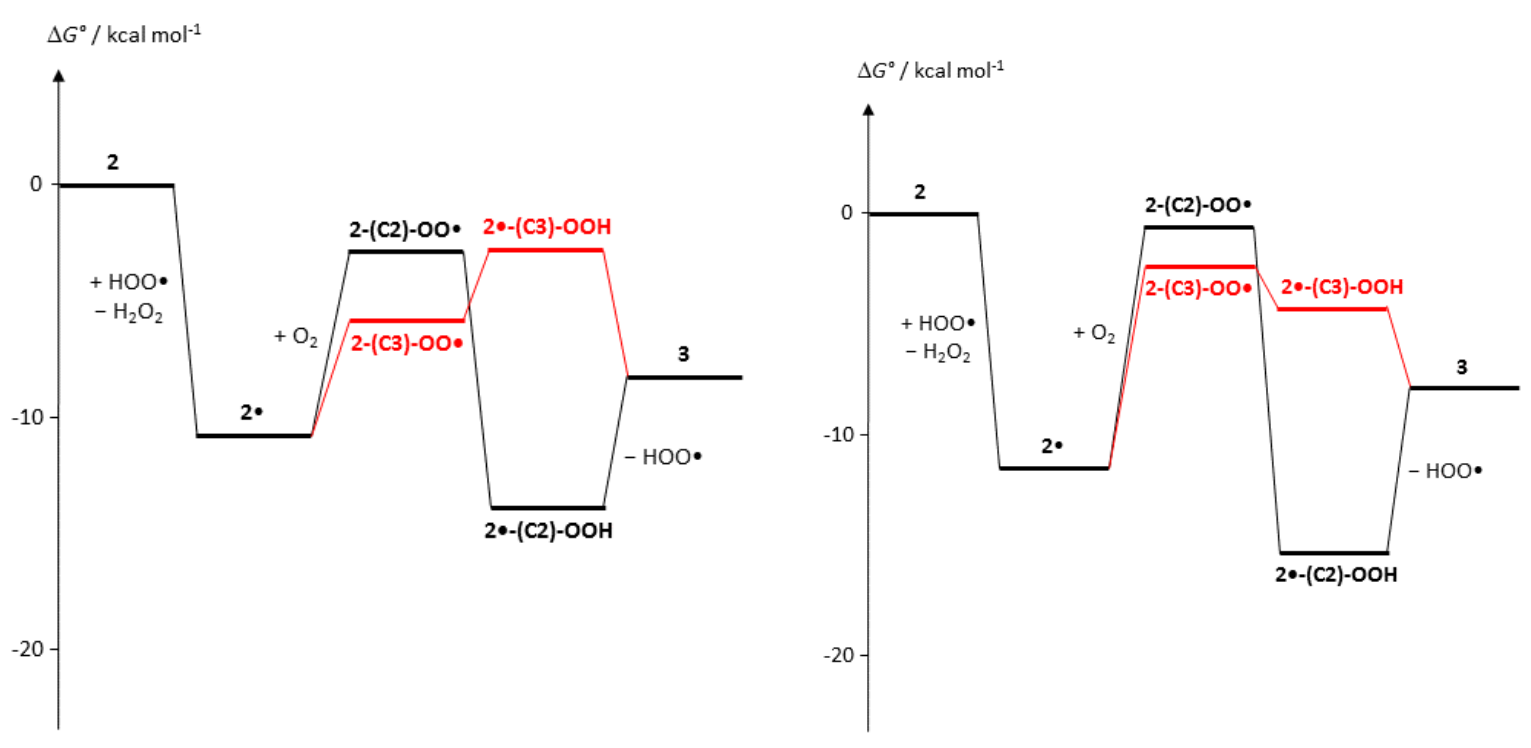

Figure S7. Computed free energy diagram for reagents, products and putative intermediates in the reaction pathway leading from $\mathbf{2}$ to $\mathbf{3}$ (reaction path a: see text), under different $\mathrm{pH}$ conditions. Left panel, $\mathrm{pH} 6.0$; right panel, $\mathrm{pH} 0.0$. The graphs at $\mathrm{pH} 3.0$ and -1.5 are shown in the main text. 

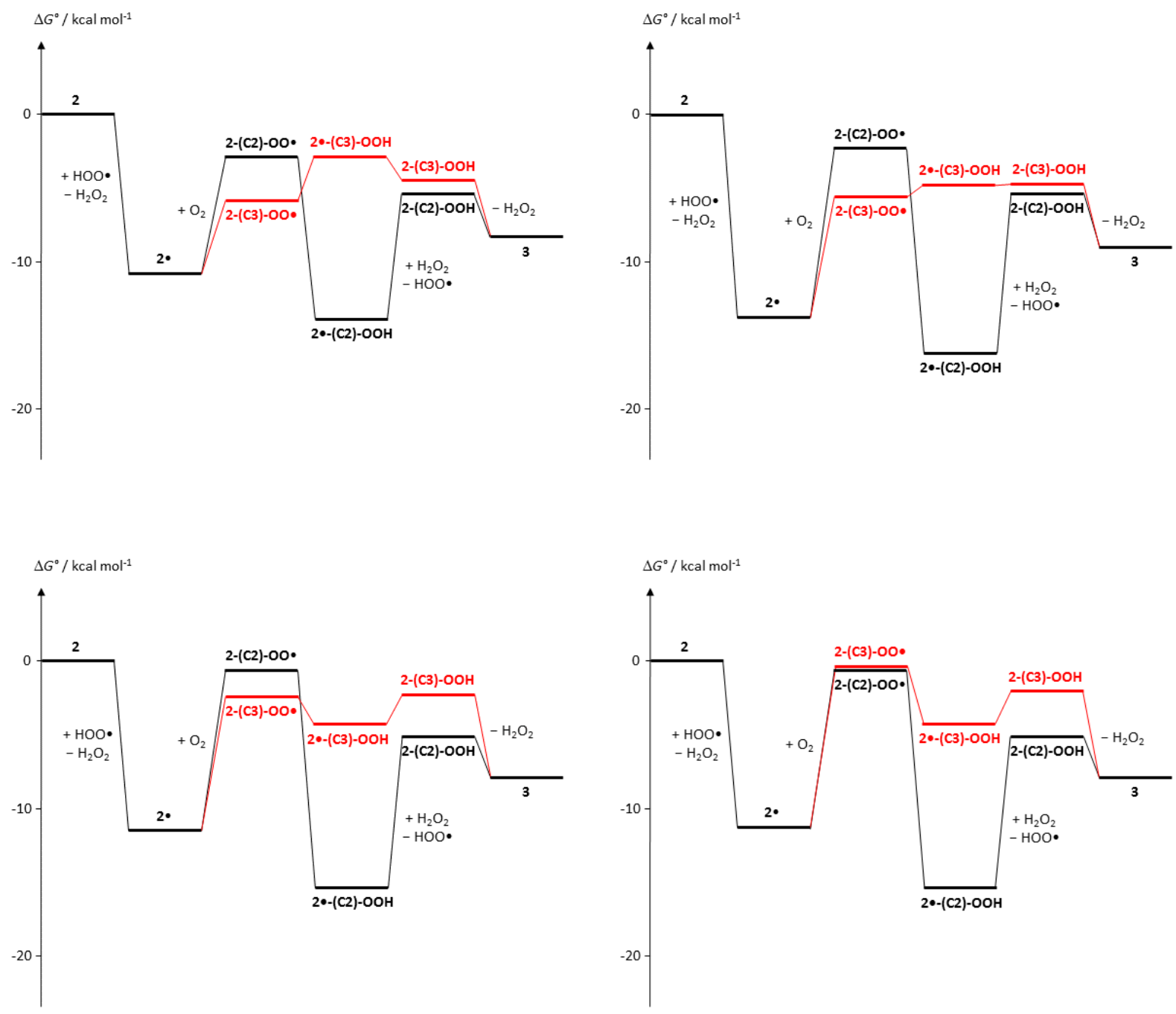

Figure 58. Computed free energy diagram for reagents, products and putative intermediates in the reaction pathway leading from $\mathbf{2}$ to $\mathbf{3}$ (reaction path b: see text) under different pH conditions. Top left panel, pH 6.0; top right panel, pH 3.0; bottom left panel, pH 0.0; bottom right panel, pH -1.5. 
Table S1. EPR parameters computed for the predominant forms of $\mathrm{N}$-monoprotonated $\mathrm{C}$-radicals from single-bond dimers in $\mathrm{MeOH}$.

\begin{tabular}{|c|c|c|c|c|}
\hline SOMOa $^{a}$ & Spin density ${ }^{b}$ & Statistical weight & $\begin{array}{l}\text { hcc / Gauss' } \\
\text { B3LYP/EPR-II }\end{array}$ & $\begin{array}{l}\text { hcc / Gauss }{ }^{2} \\
\text { B3LYP/EPR-III }\end{array}$ \\
\hline & & 0.36 & 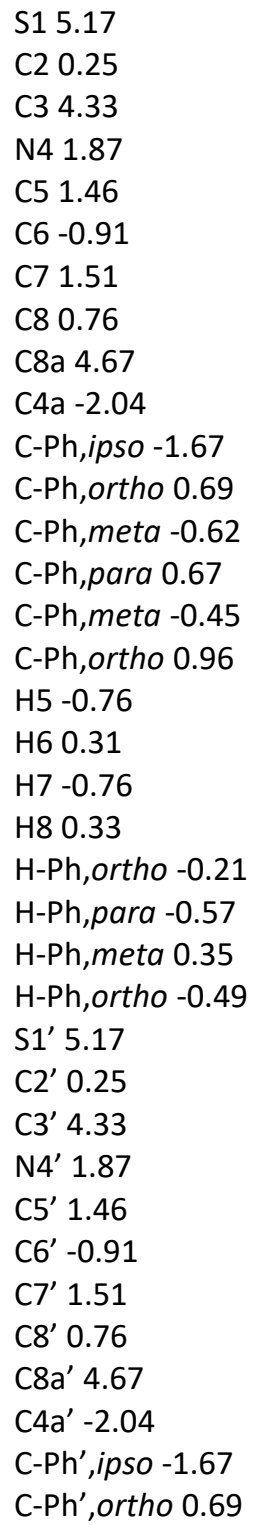 & 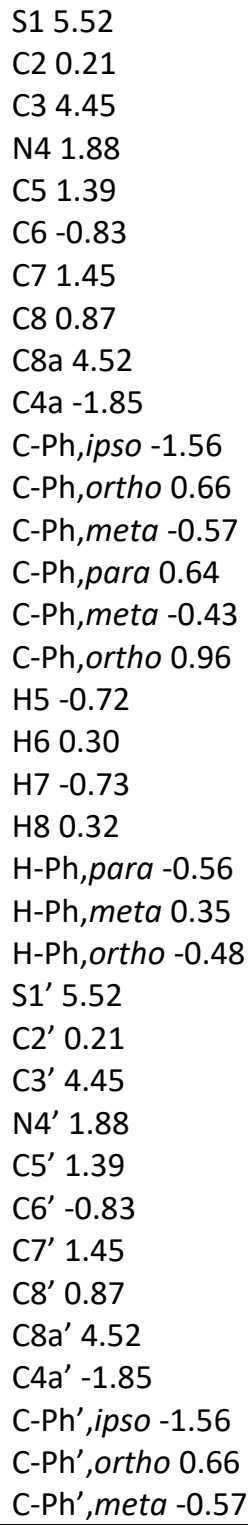 \\
\hline
\end{tabular}




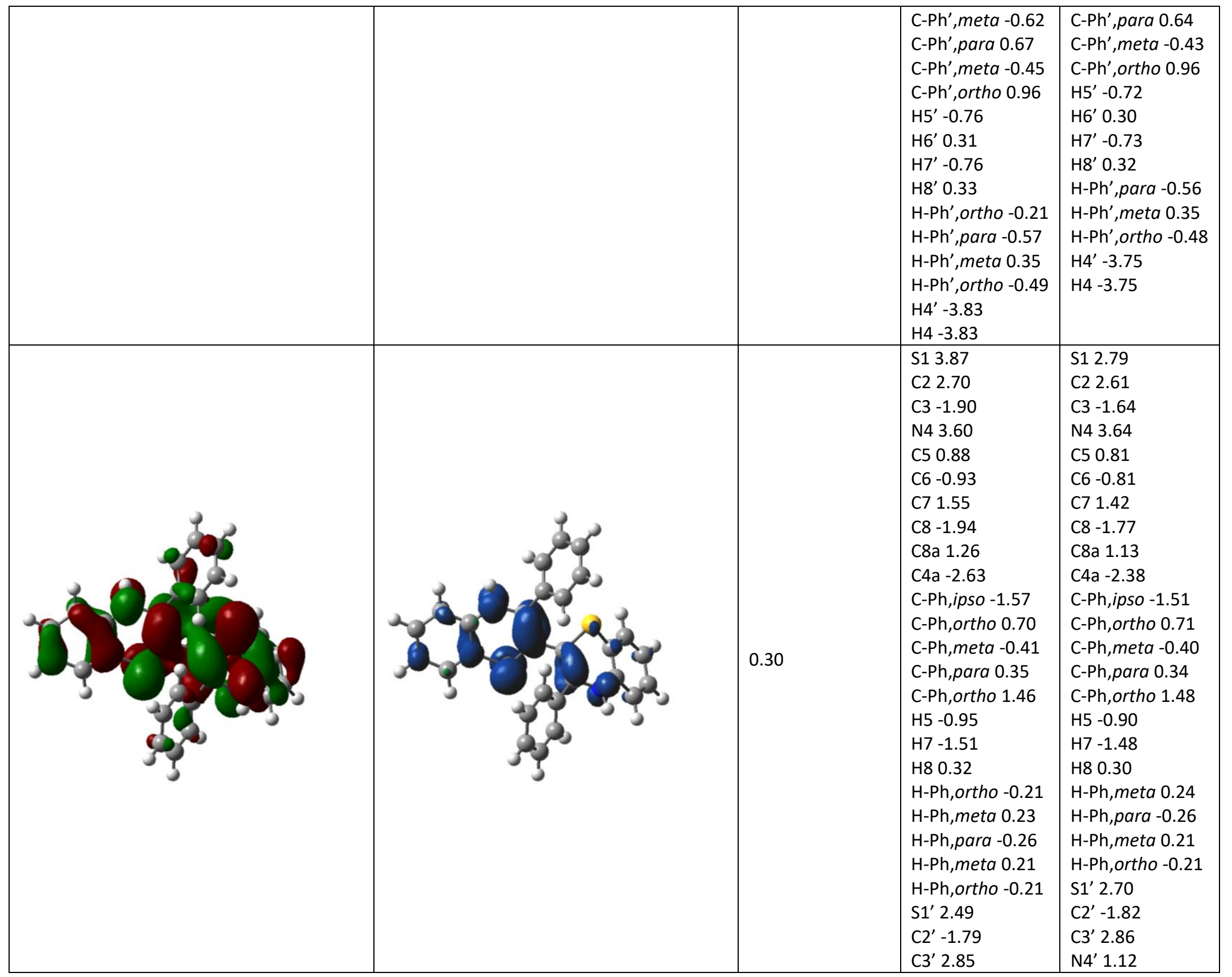




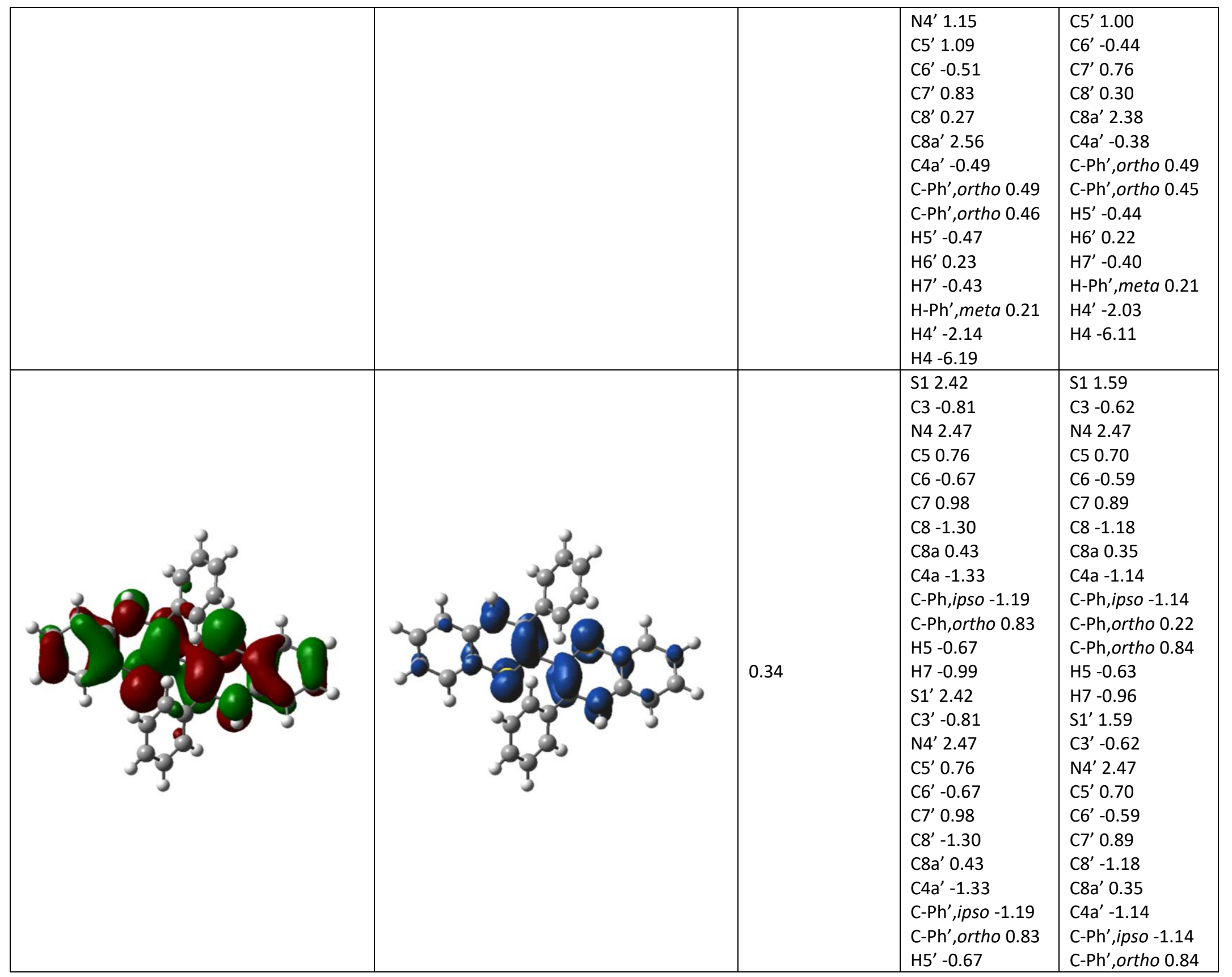




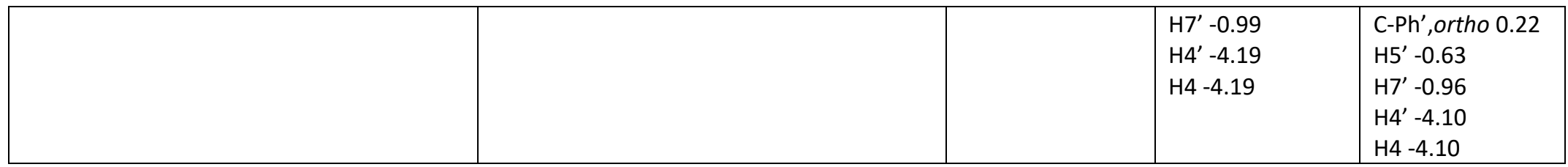

[a] Isovalue 0.02 a.u.

[b] Isodensity 0.002 a.u.

[c] Isotopes: ${ }^{1} \mathrm{H},{ }^{13} \mathrm{C},{ }^{14} \mathrm{~N},{ }^{33} \mathrm{~S}$. Only couplings whose absolute value exceeds 0.2 Gauss are listed. The numbering system is shown in the following scheme:

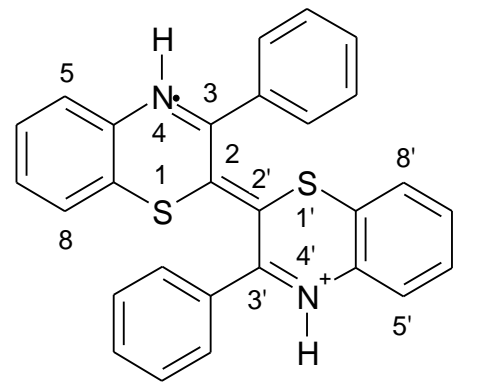


Table S2. EPR parameters computed for the predominant forms of $N, N^{\prime}$-diprotonated $C$-radicals from single-bond dimers in $M e O H$.

\begin{tabular}{|c|c|c|c|c|}
\hline SOMO $^{\mathrm{a}}$ & Spin density & Statistical weight & \begin{tabular}{|l|} 
hcc / Gauss \\
B3LYP/EPR-II
\end{tabular} & $\begin{array}{l}\text { hcc / Gauss }{ }^{c} \\
\text { B3LYP/EPR-III }\end{array}$ \\
\hline & & 0.54 & $\begin{array}{l}\text { S1 } 6.41 \\
\text { C2 } 7.78 \\
\text { C3 }-5.54 \\
\text { N4 4.11 } \\
\text { C5 }-0.41 \\
\text { C7 } 1.46 \\
\text { C8 }-2.13 \\
\text { C8a } 1.32 \\
\text { C4a }-2.73 \\
\text { C-Ph ortho } 0.34 \\
\text { C-Ph ortho }-0.29 \\
\text { H4 -6.99 } \\
\text { H5 -0.65 } \\
\text { H6 -0.81 } \\
\text { H7 -1.74 } \\
\text { S1' } 1.36 \\
\text { C2' -4.17 } \\
\text { C3' } 2.41 \\
\text { C5' }-0.26 \\
\text { C6' } 0.30 \\
\text { C7' }-0.30 \\
\text { C8' } 0.46 \\
\text { C8a' }-0.29 \\
\text { C4a } 0.32 \\
\text { C-Ph', ipso }-0.29 \\
\text { C-Ph',ortho } 0.22 \\
\text { H2' } 0.57 \\
\text { H4' } 0.51 \\
\text { H6' }-0.22 \\
\text { H8' }-0.27 \\
\end{array}$ & 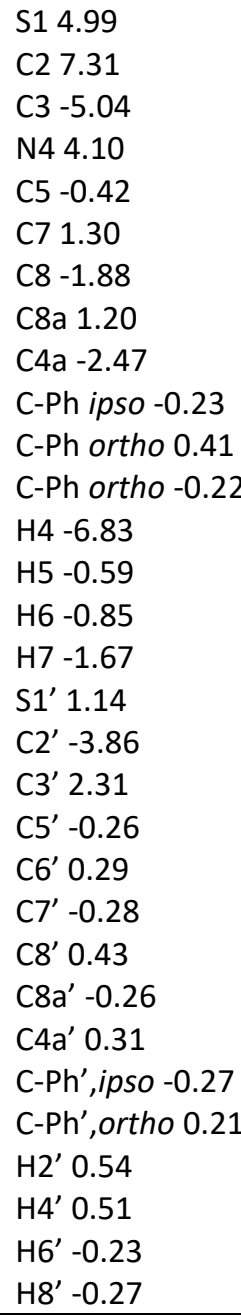 \\
\hline
\end{tabular}




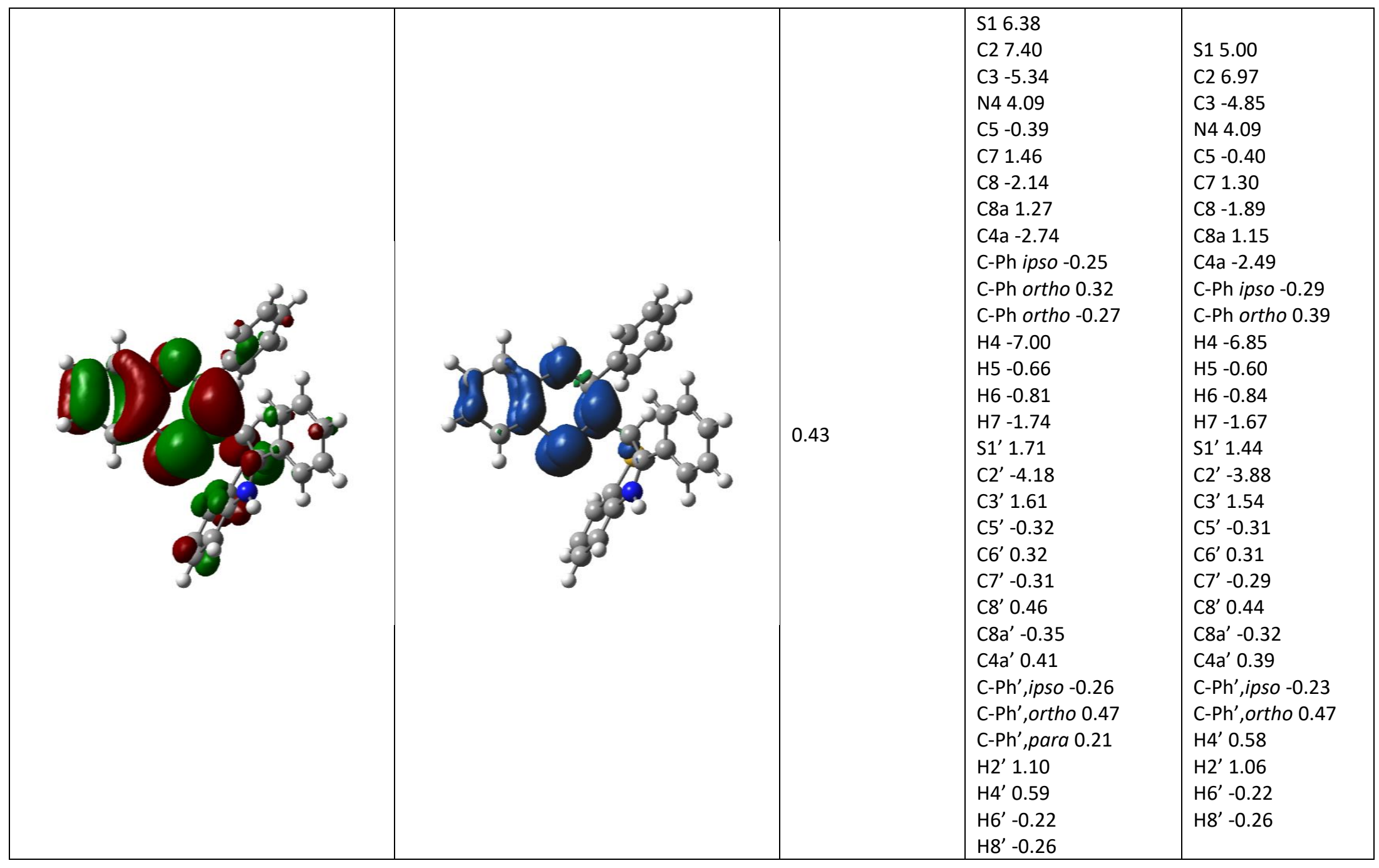




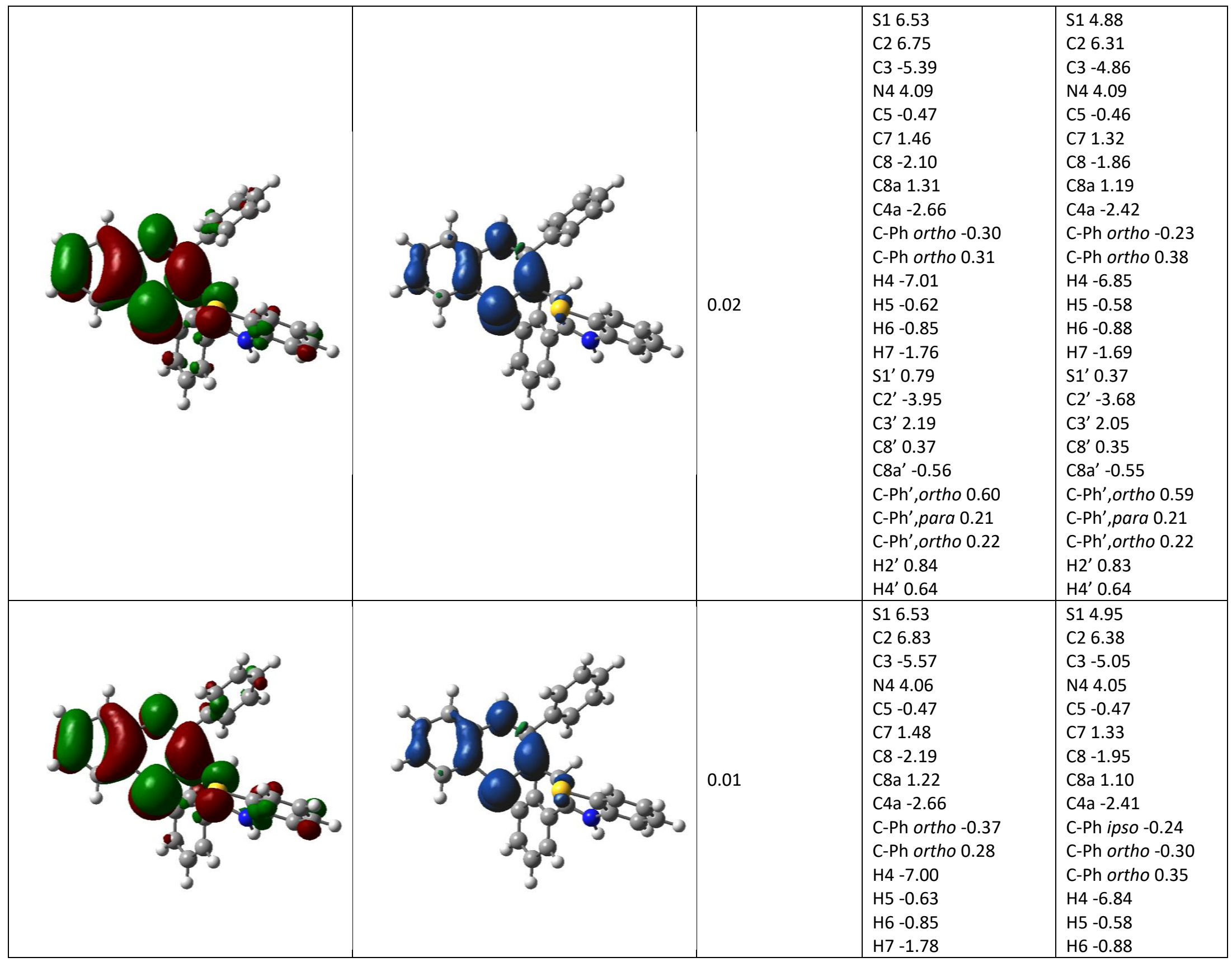




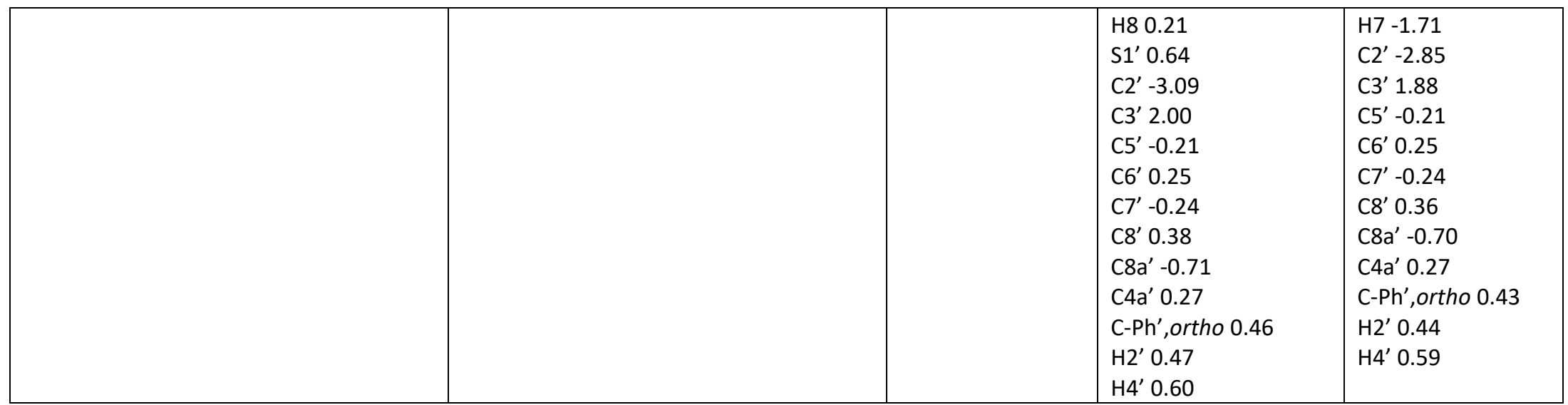

[a] Isovalue 0.02 a.u.

[b] Isodensity 0.002 a.u.

[c] Isotopes: ${ }^{1} \mathrm{H},{ }^{13} \mathrm{C},{ }^{14} \mathrm{~N},{ }^{33} \mathrm{~S}$. Only couplings whose absolute value exceeds 0.2 Gauss are listed. The numbering system is shown in the following scheme:

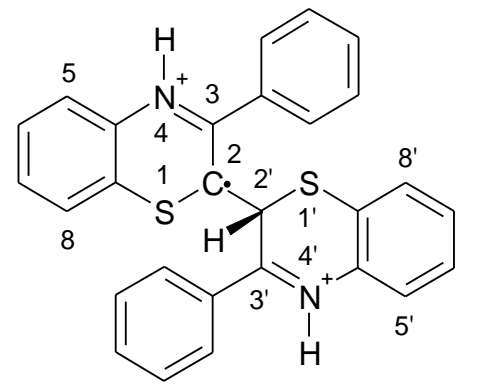


Table S3. Neutral forms in vacuo. For each chemical species, values are reported for the most stable conformer / geometric isomer identified. $G_{R R H O}$,alc $[\mathrm{M062X} / 6-311++\mathrm{G}(2 \mathrm{~d}, 2 \mathrm{p})]=E_{\mathrm{el}}[\mathrm{M062X} / 6-311++\mathrm{G}(2 \mathrm{~d}, 2 \mathrm{p}) / / \mathrm{PBE} 0 / 6-31+\mathrm{G}(\mathrm{d}, \mathrm{p})]-E_{\text {el }}[\mathrm{PBE0} / 6-31+\mathrm{G}(\mathrm{d}, \mathrm{p})]+G_{\mathrm{RRHO}}[\mathrm{PBE} 0 / 6-31+\mathrm{G}(\mathrm{d}, \mathrm{p})] ;$ and likewise for $G_{\text {RRHO, calc }}[M 062 X / 6-31+G(d, p)]$. In this and in the following tables, $G$ values are referred to a 1 atm standard state; however, they were converted to a $1 \mathrm{M}$ standard state in order to compute the reaction free energies discussed in the paper (see e.g. Cramer, C. J. Essentials of Computational Chemistry: Theories and Models, 2nd edition, John Wiley \& Sons, Chichester, 2004, pp 378-379).

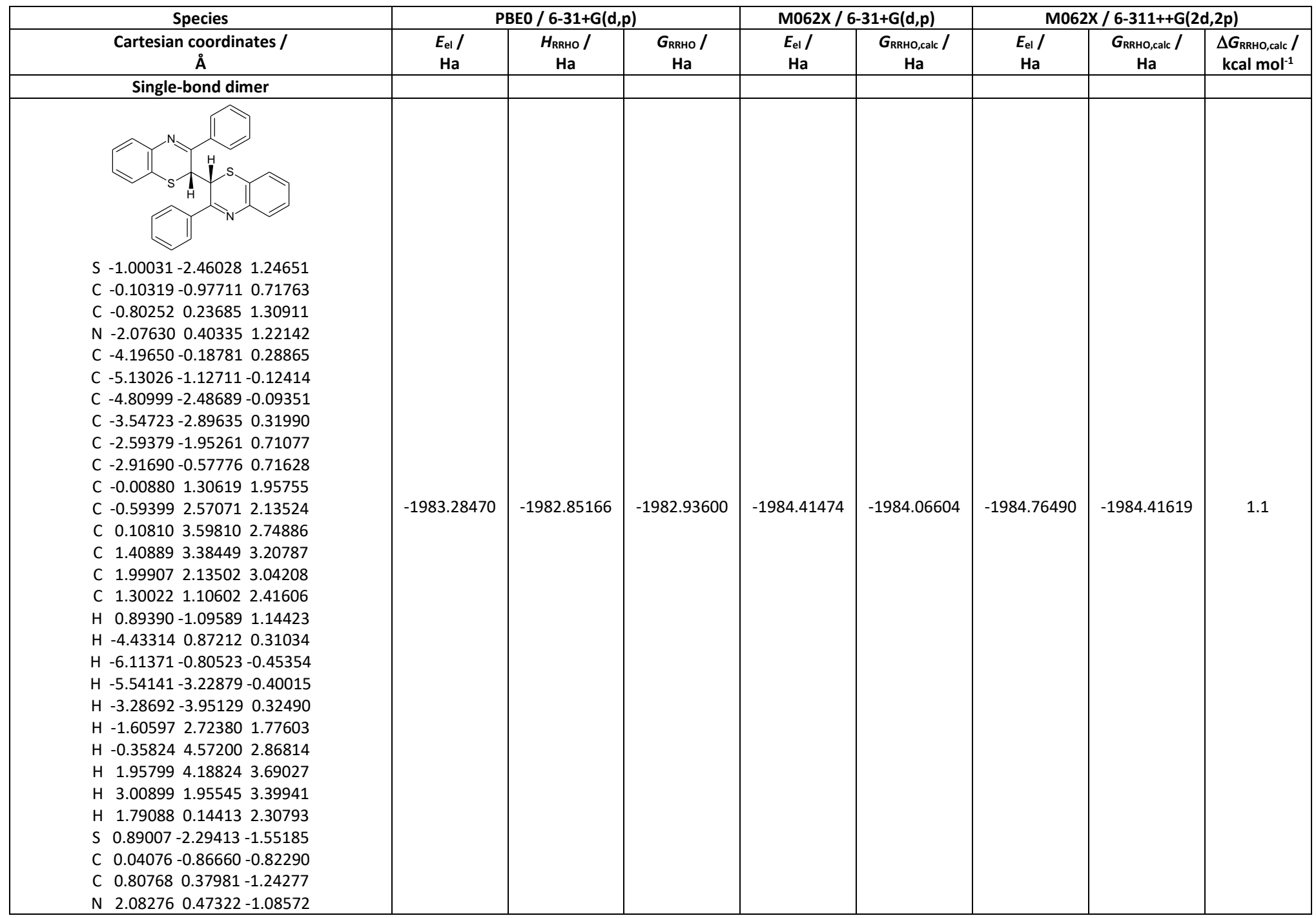




\begin{tabular}{|c|c|c|c|c|c|c|c|c|}
\hline $\begin{array}{lllll}\text { C } & 4.12949 & -0.33778 & -0.15019 \\
\text { C } & 5.00671 & -1.36905 & 0.15161 \\
\text { C } & 4.63928 & -2.69373 & -0.10147 \\
\text { C } & 3.38228 & -2.98089 & -0.62153 \\
\text { C } & 2.48309 & -1.94775 & -0.89902 \\
\text { C } & 2.85885 & -0.60279 & -0.68618 \\
\text { C } & 0.09495 & 1.53755 & -1.83178 \\
\text { C } & 0.84521 & 2.63946 & -2.27646 \\
\text { C } & 0.22178 & 3.74425 & -2.83744 \\
\text { C } & -1.16788 & 3.77610 & -2.97020 \\
\text { C } & -1.92320 & 2.69229 & -2.53340 \\
\text { C } & -1.29899 & 1.58212 & -1.96931 \\
\text { H } & -0.95166 & -0.88687 & -1.27626 \\
\text { H } & 4.40384 & 0.70176 & 0.00369 \\
\text { H } & 5.98462 & -1.14434 & 0.56707 \\
\text { H } & 5.32824 & -3.50445 & 0.11649 \\
\text { H } & 3.08291 & -4.01068 & -0.79661 \\
\text { H } & 1.92392 & 2.60199 & -2.16888 \\
\text { H } & 0.82025 & 4.58535 & -3.17609 \\
\text { H } & -1.65605 & 4.64015 & -3.41224 \\
\text { H } & -3.00514 & 2.70555 & -2.62905 \\
\text { H } & -1.92341 & 0.76338 & -1.62805 \\
\end{array}$ & & & & & & & & \\
\hline 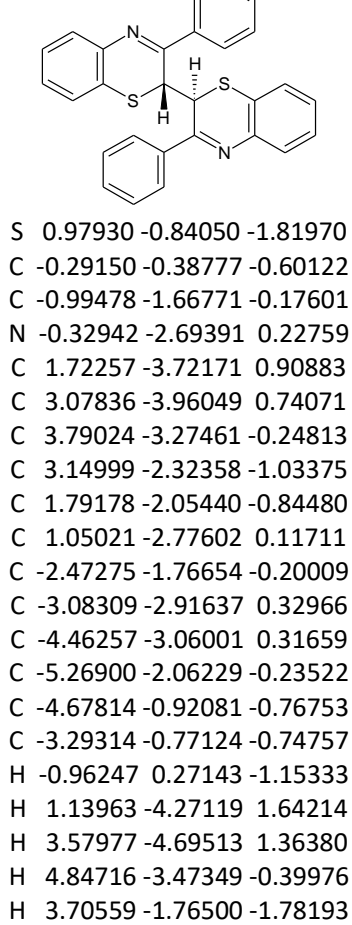 & -1983.28475 & -1982.85171 & -1982.93580 & -1984.41618 & -1984.06723 & -1984.76686 & -1984.41791 & 0.0 \\
\hline
\end{tabular}




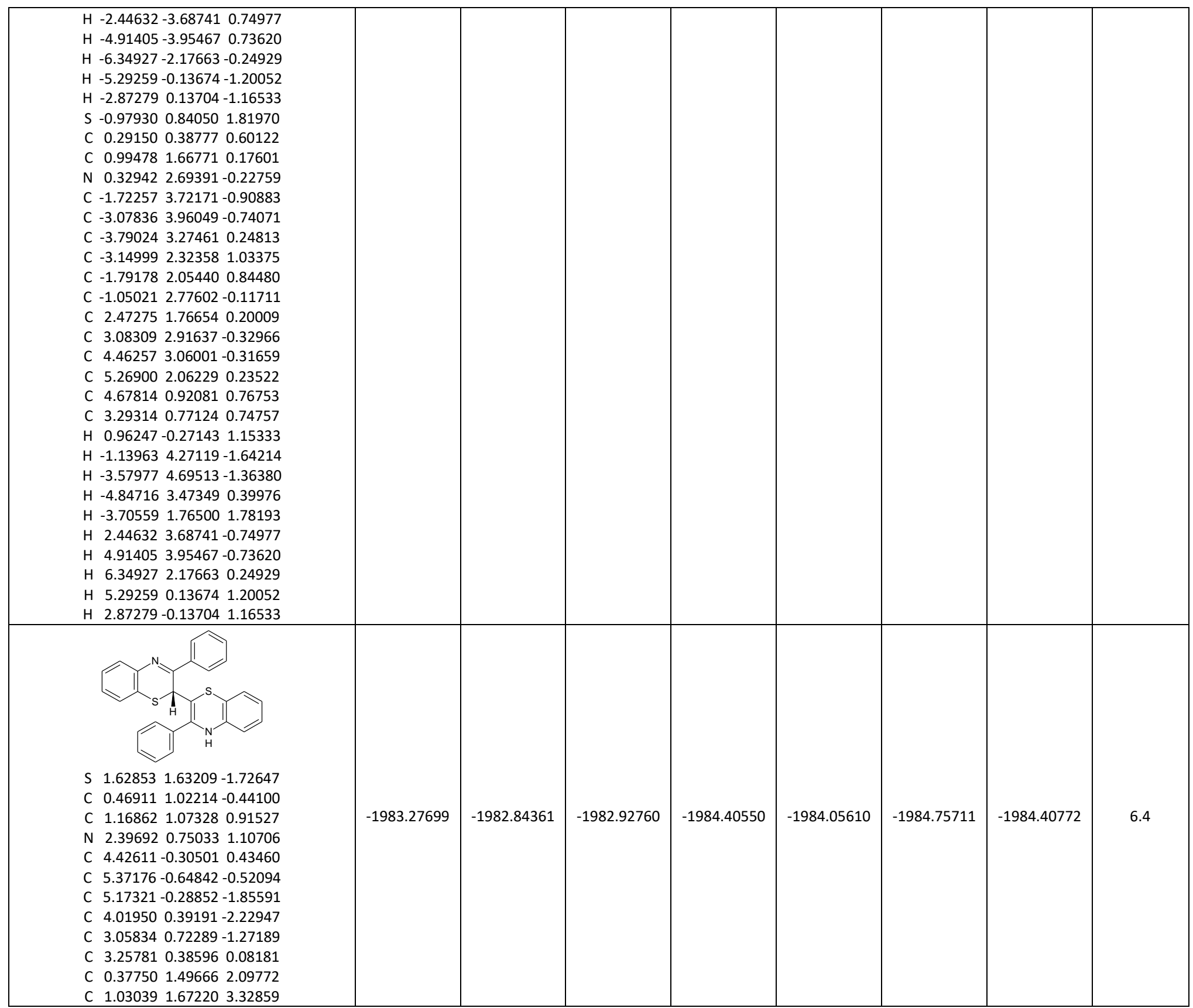




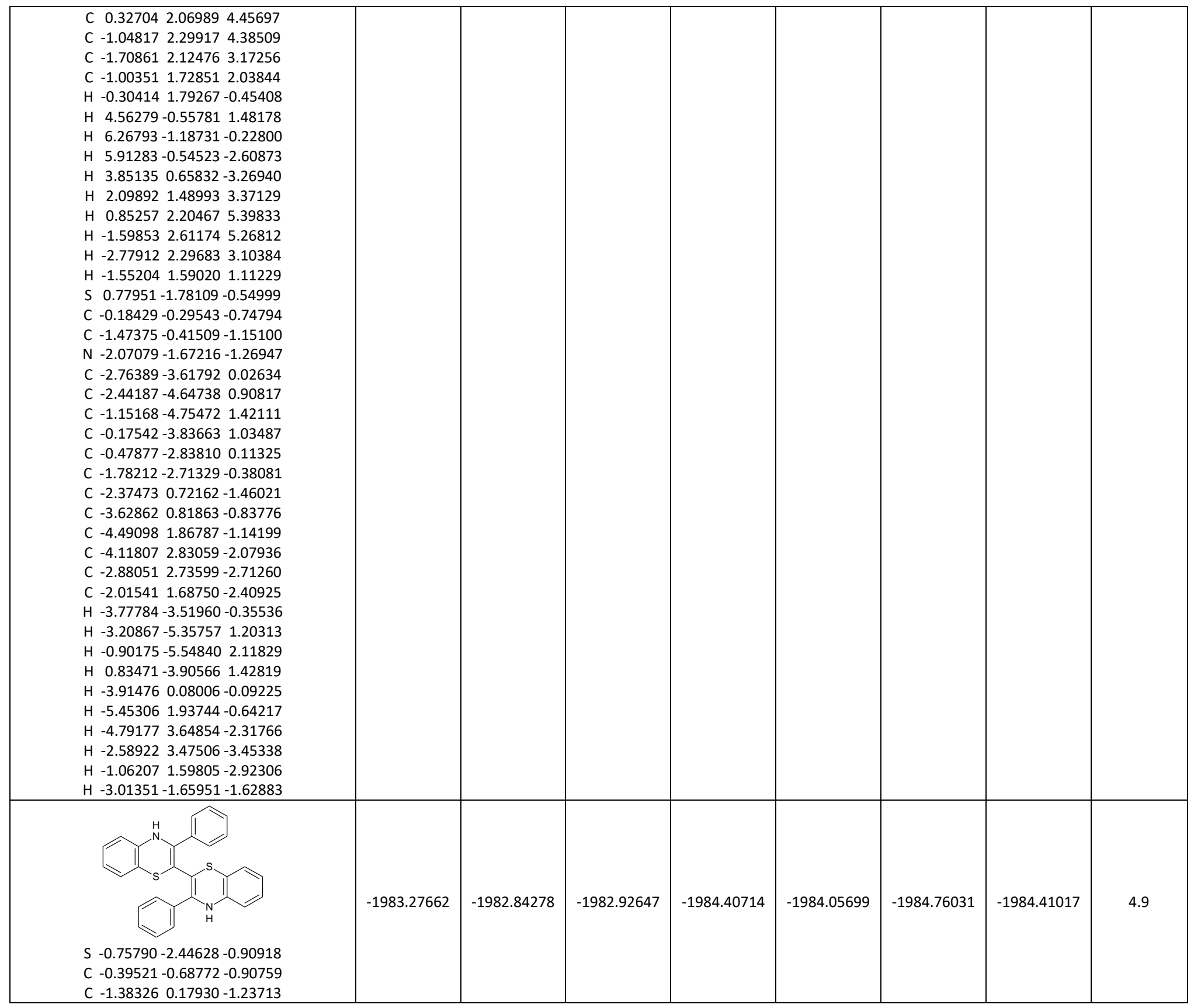




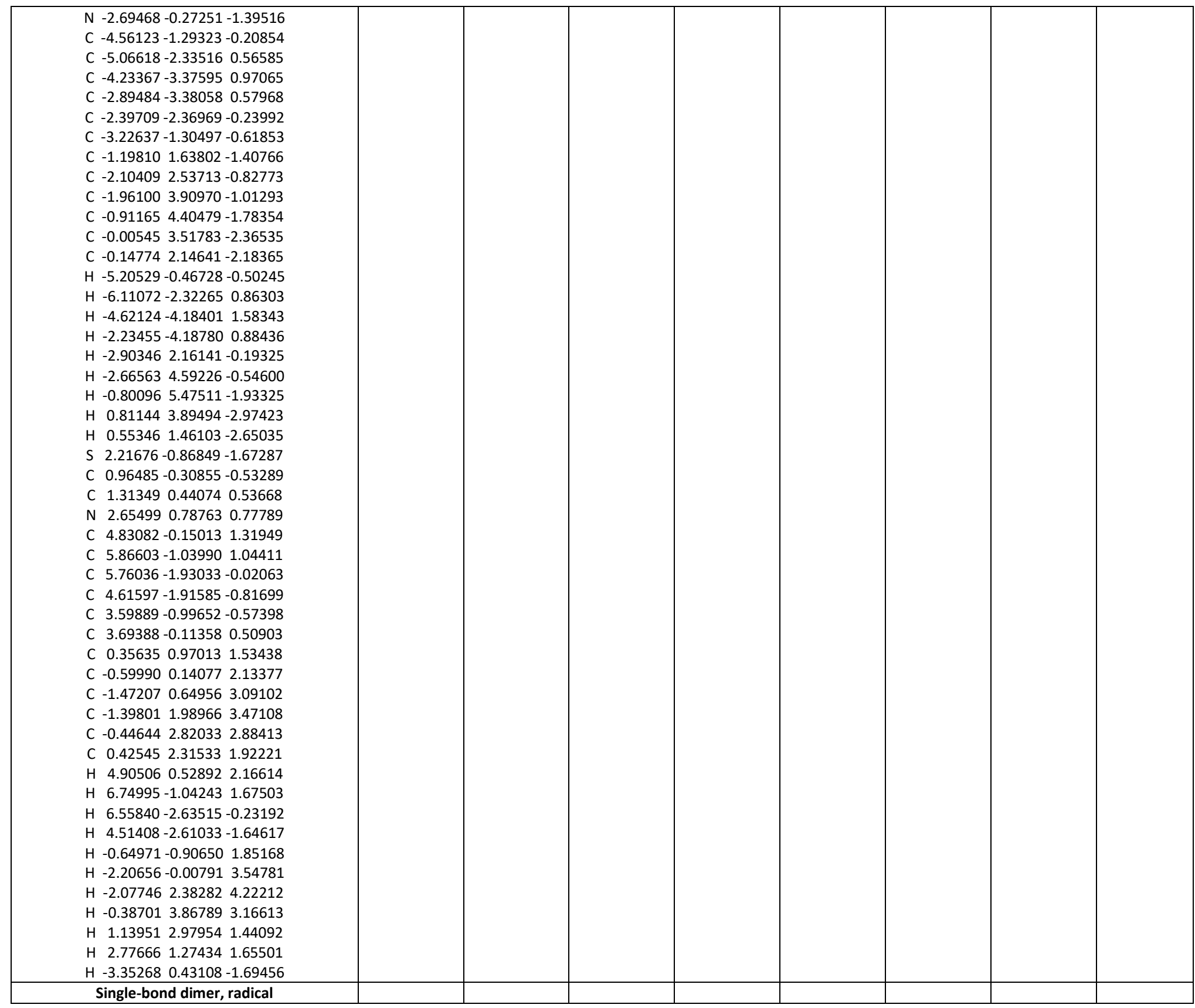




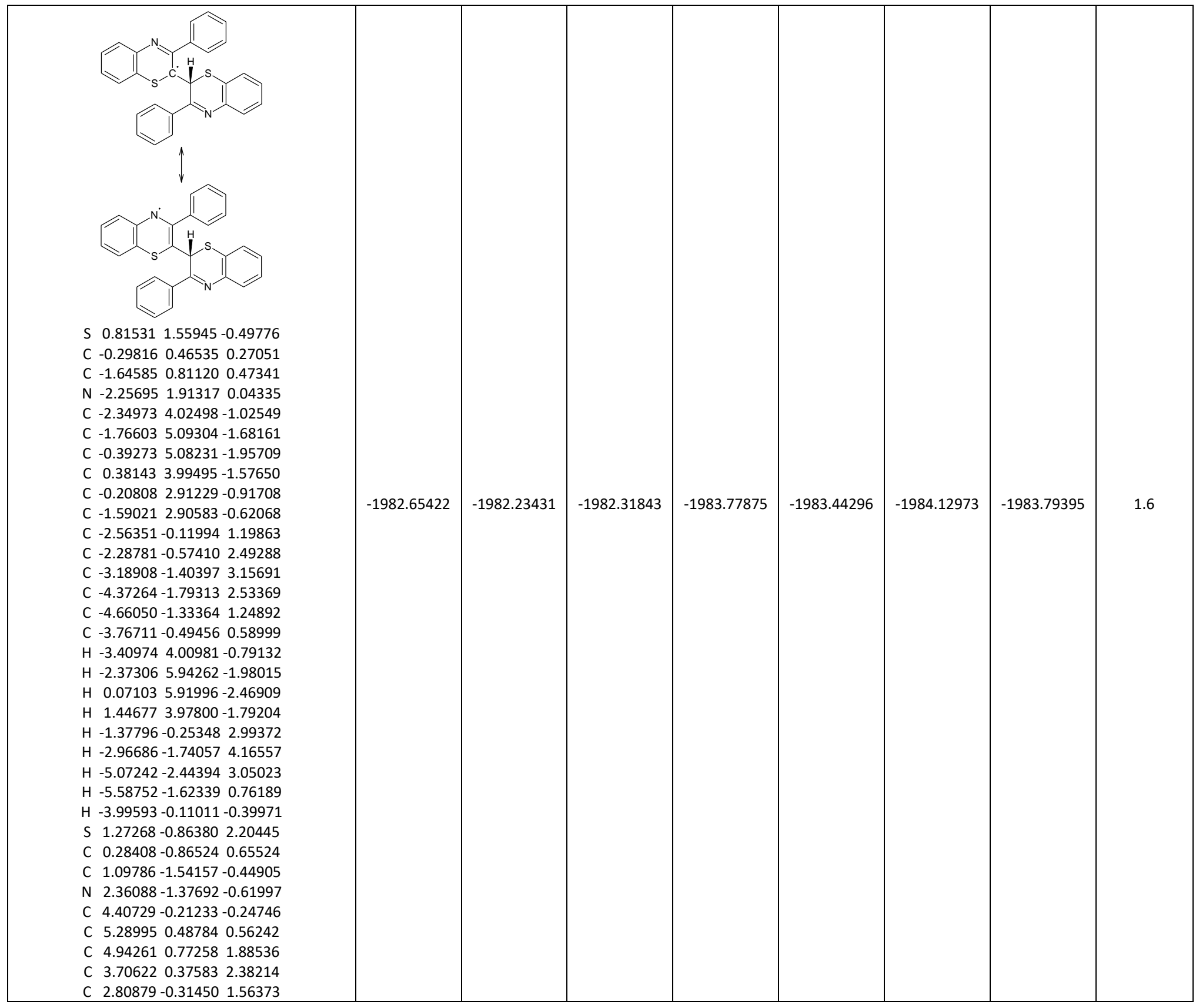




\begin{tabular}{|c|c|c|c|c|c|c|c|c|}
\hline 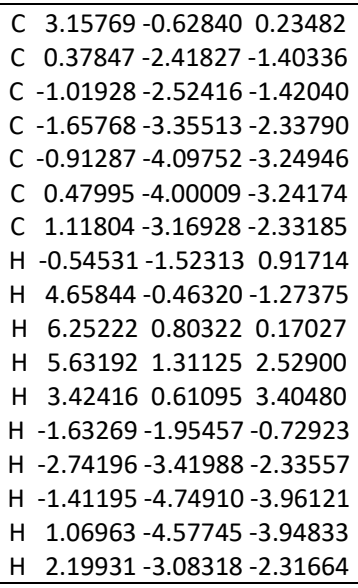 & 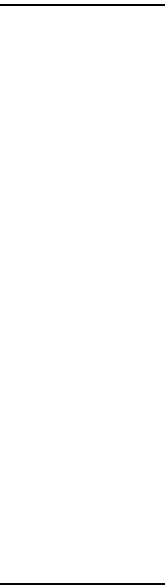 & 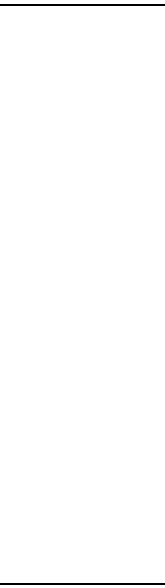 & & & & 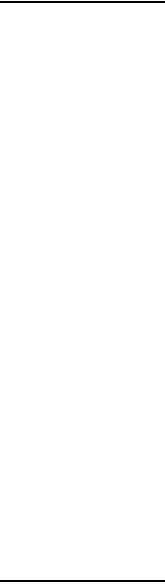 & 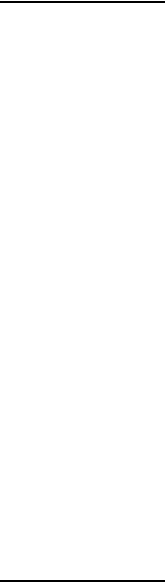 & \\
\hline 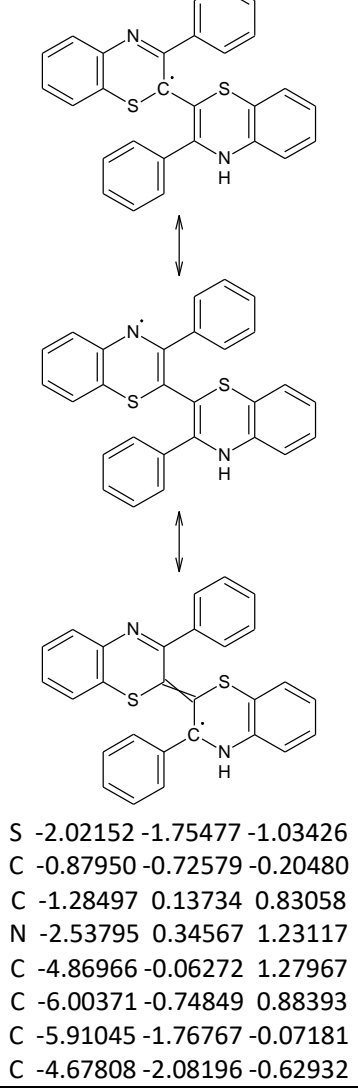 & -1982.65389 & -1982.23380 & -1982.31803 & -1983.78060 & -1983.44474 & -1984.13235 & -1983.79650 & 0.0 \\
\hline
\end{tabular}




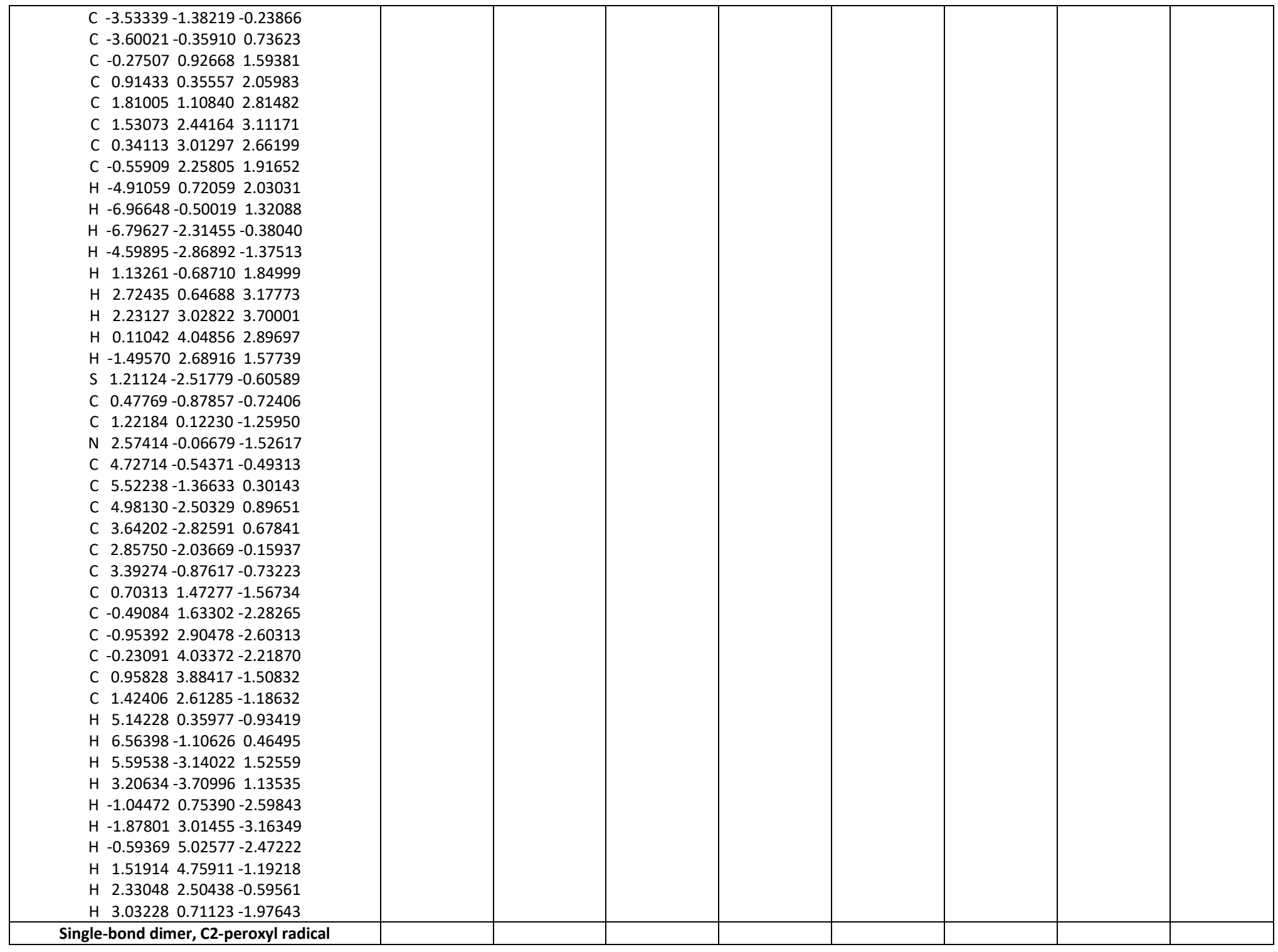




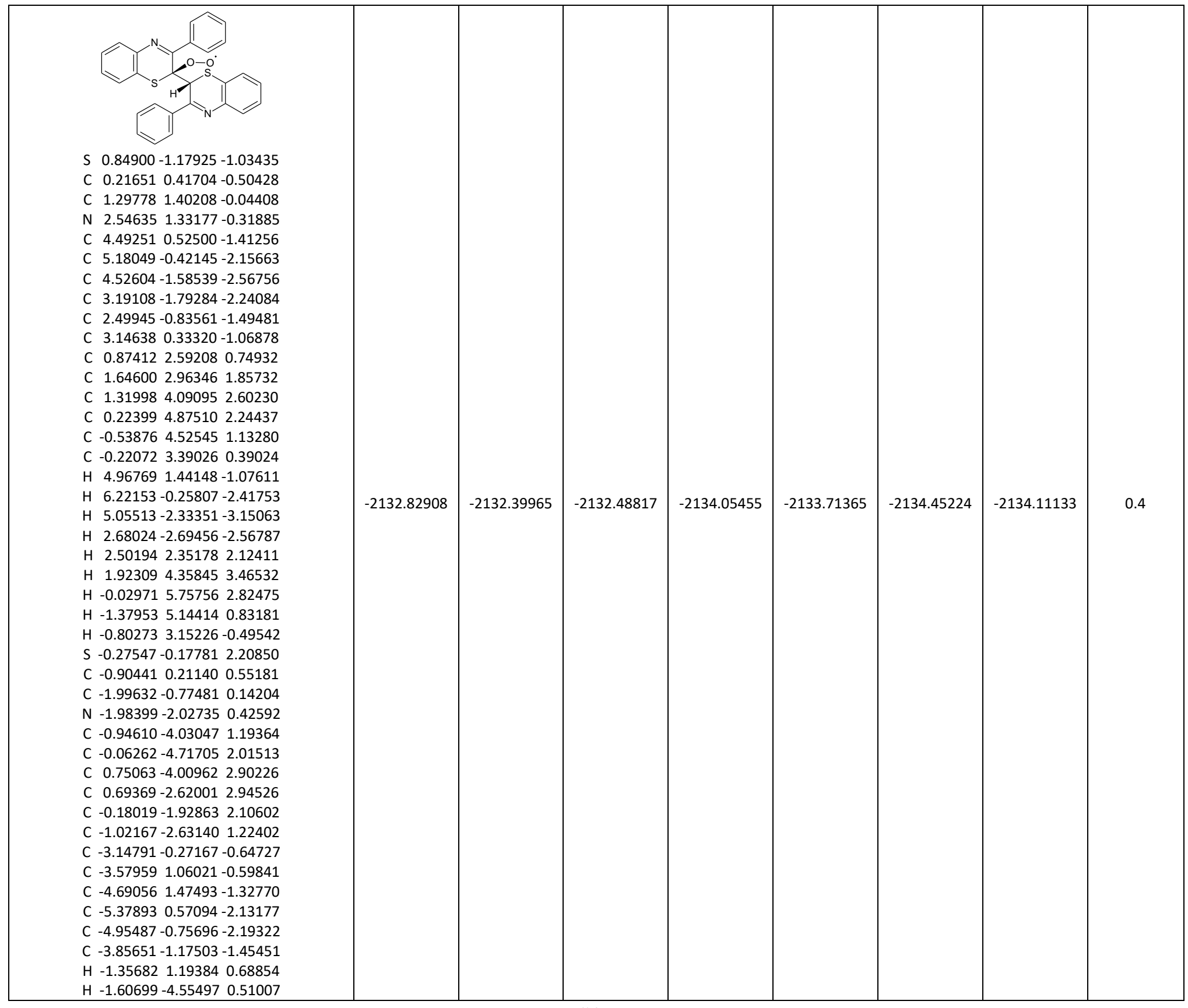




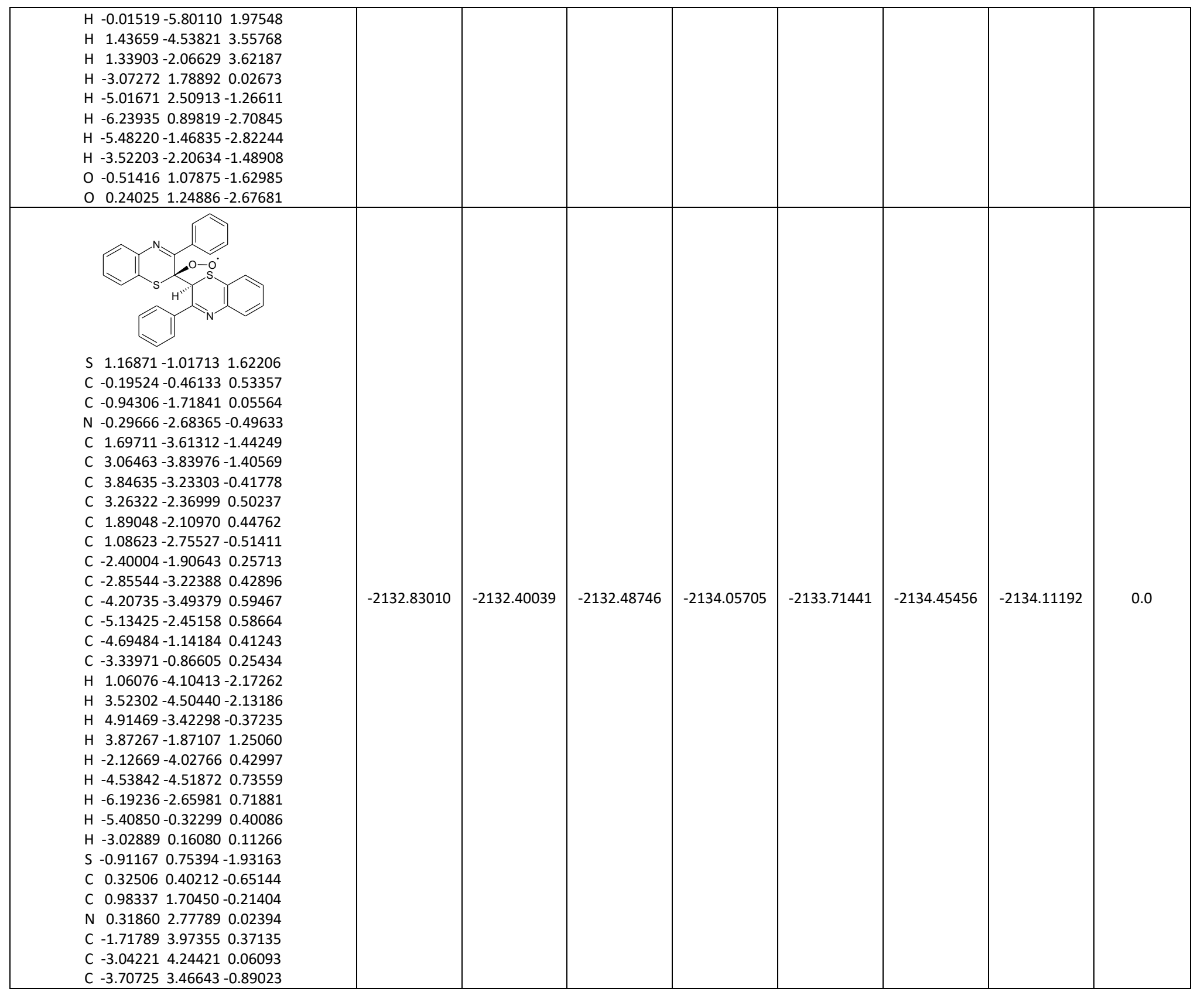




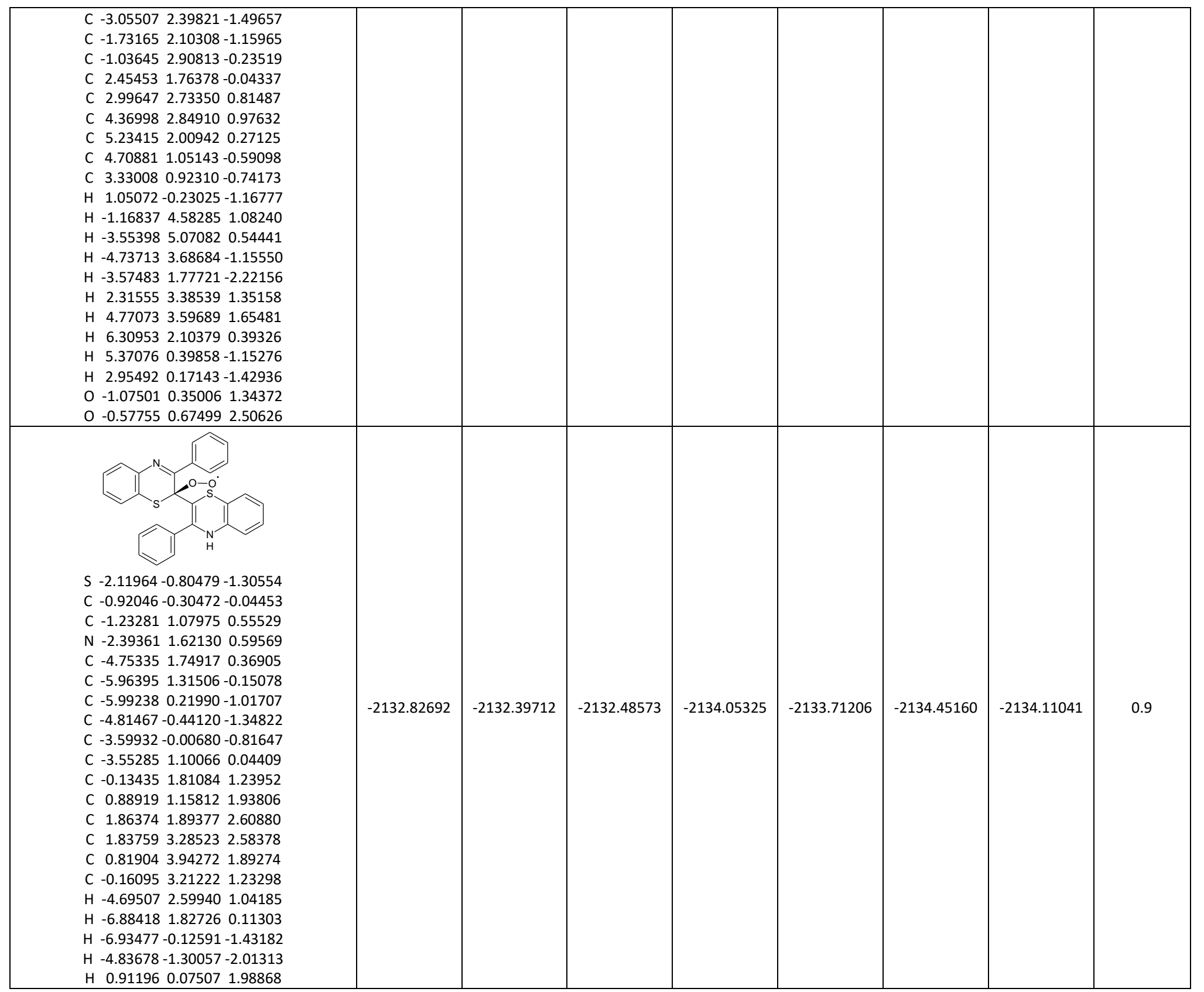




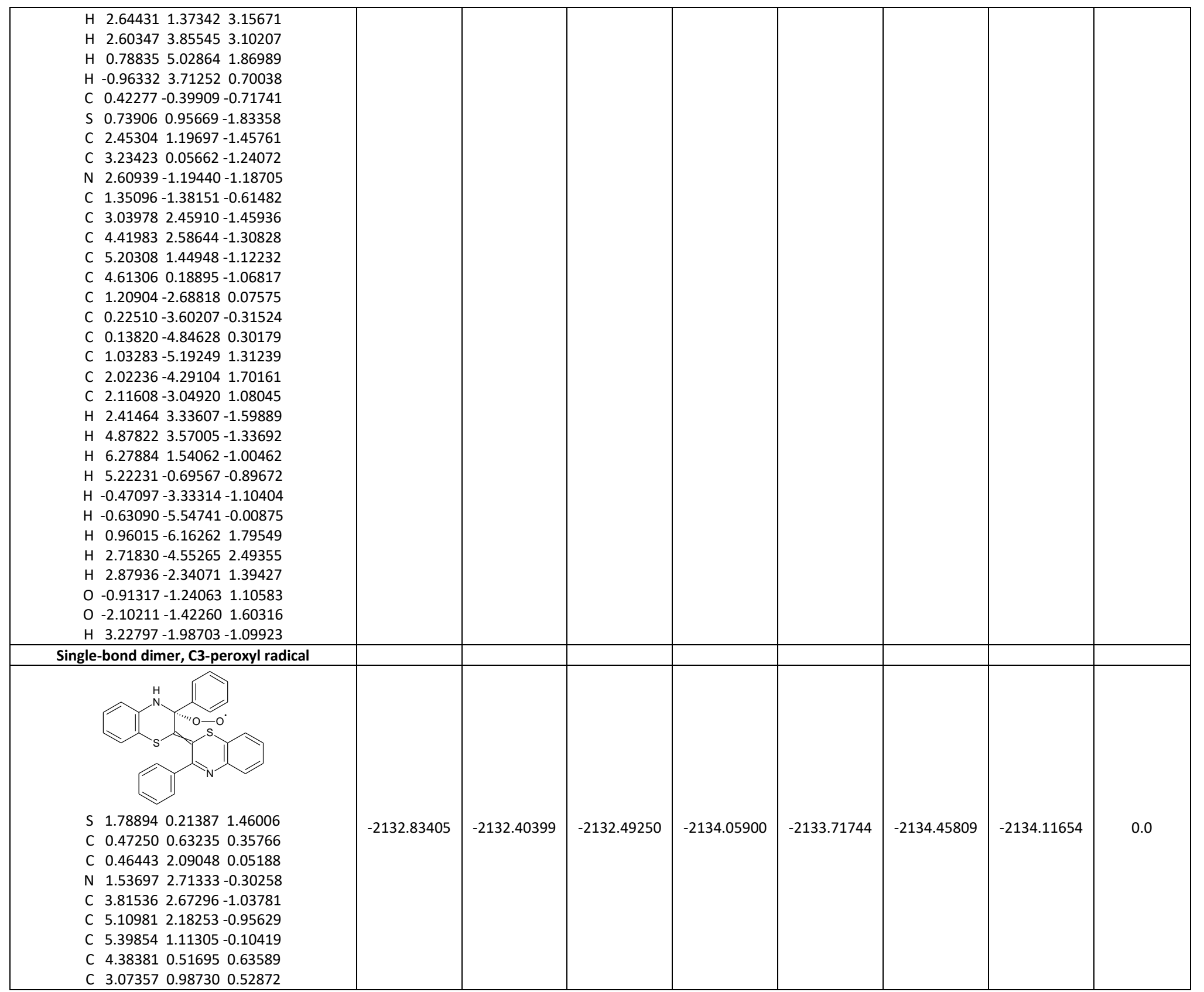




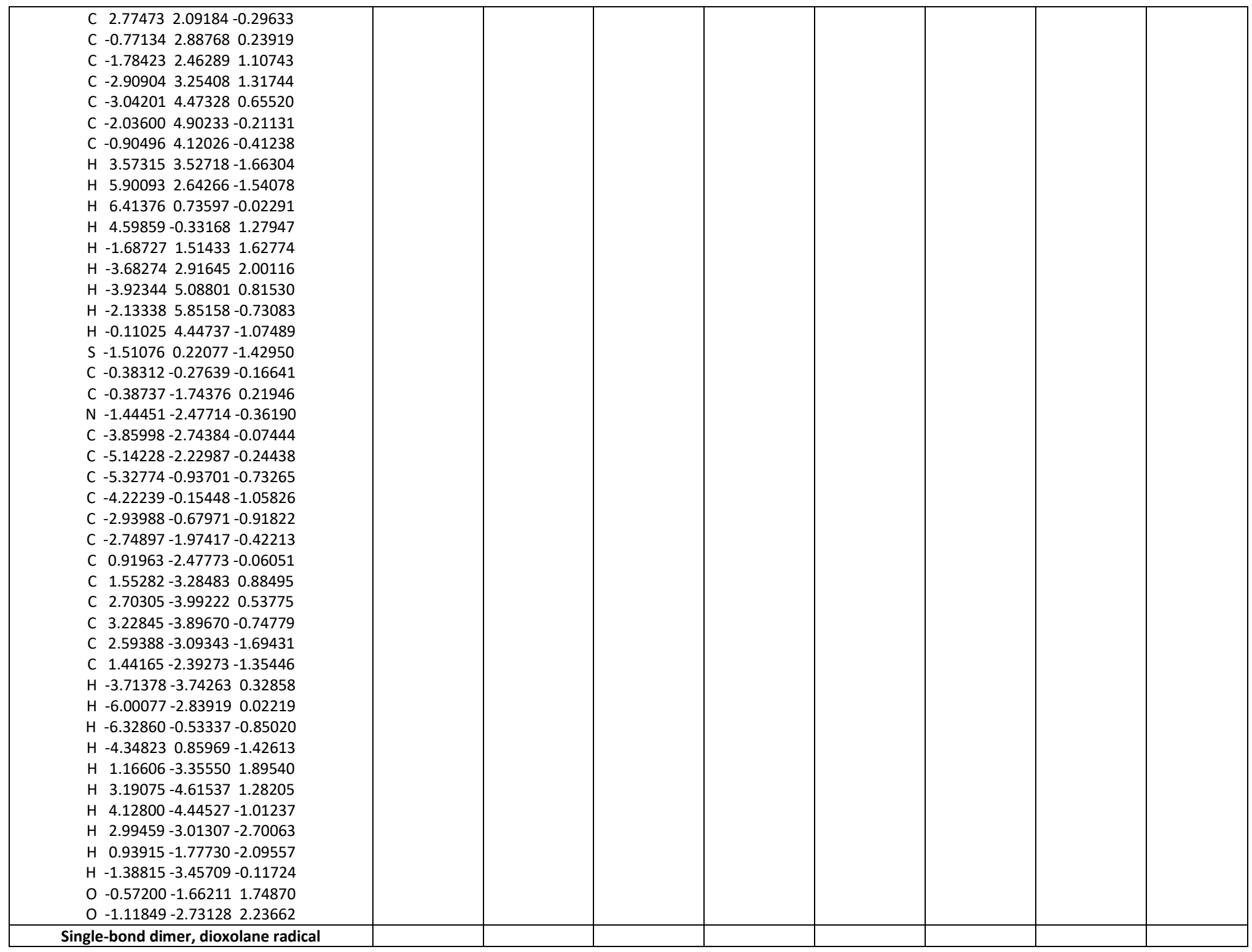




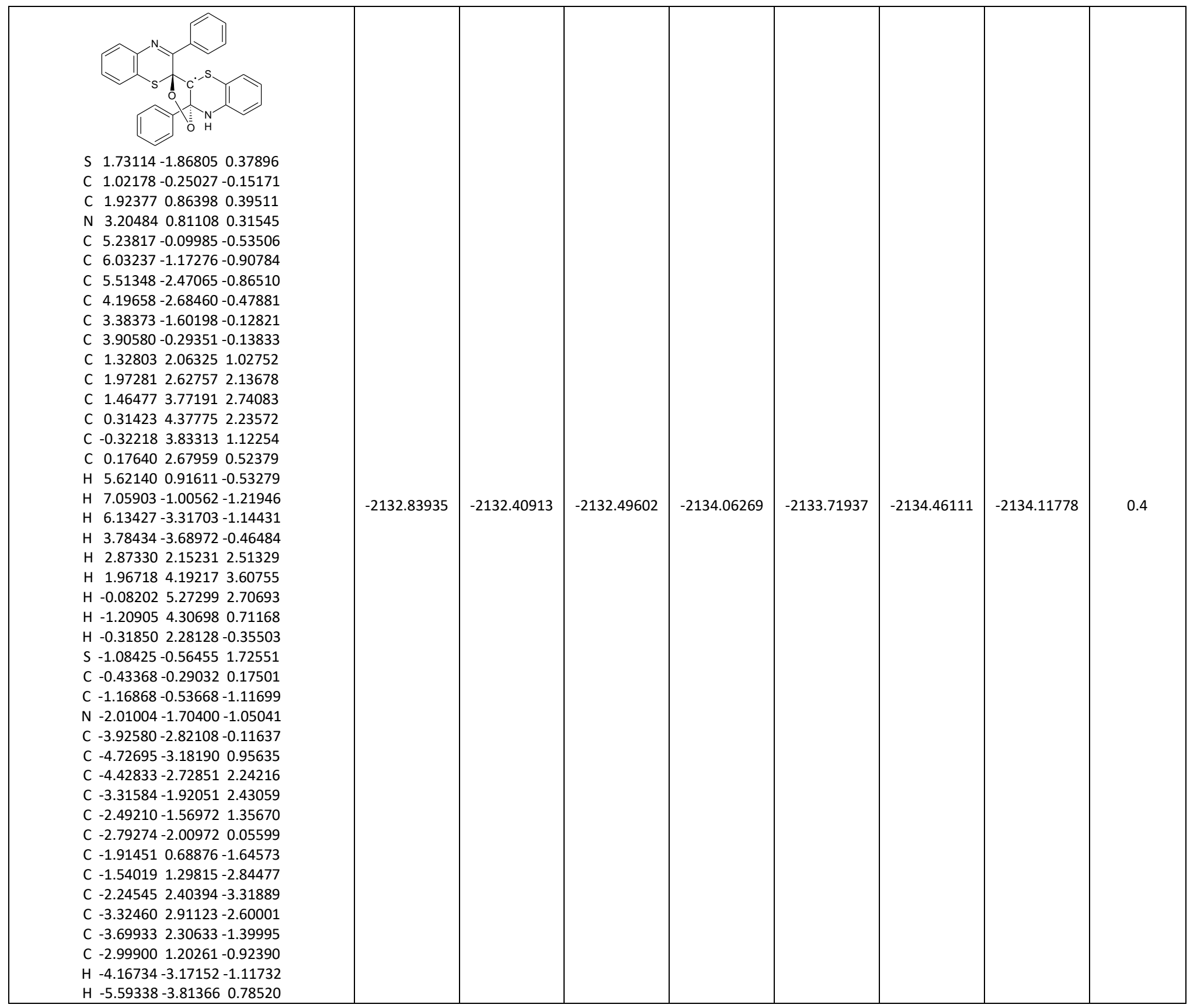




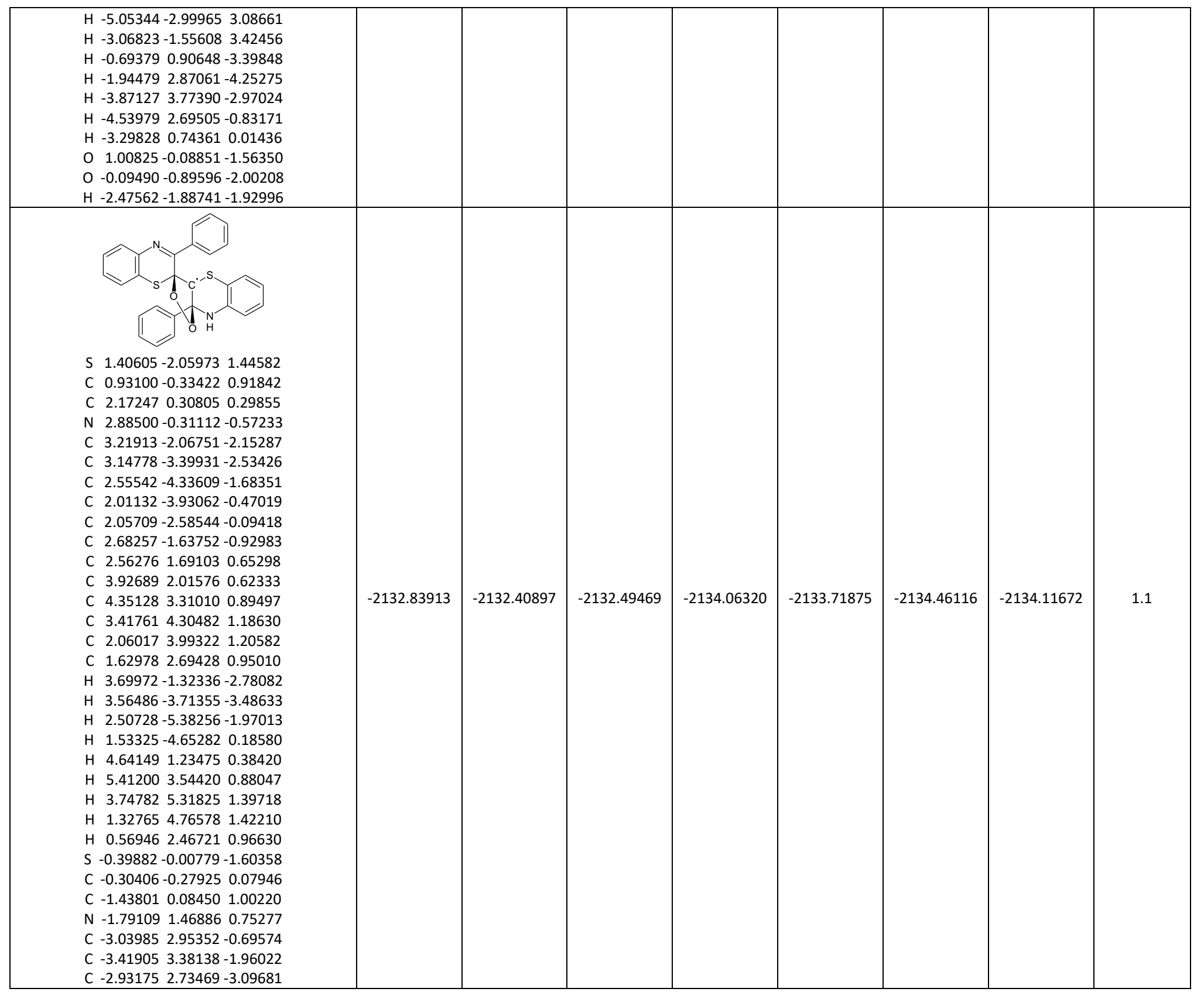




\begin{tabular}{|c|c|c|c|c|c|c|c|c|}
\hline $\begin{array}{llll}\text { C } & -2.04630 & 1.67597 & -2.94773 \\
\text { C } & -1.63876 & 1.25545 & -1.67860 \\
\text { C } & -2.15459 & 1.87820 & -0.52676 \\
\text { C } & -2.61153 & -0.88958 & 1.02187 \\
\text { C } & -2.37037 & -2.23503 & 1.31982 \\
\text { C } & -3.42021 & -3.14497 & 1.35369 \\
\text { C } & -4.72305 & -2.72441 & 1.08454 \\
\text { C } & -4.96678 & -1.38861 & 0.78243 \\
\text { C } & -3.91511 & -0.47277 & 0.75199 \\
\text { H } & -3.43427 & 3.44983 & 0.18863 \\
\text { H } & -4.10670 & 4.21620 & -2.05708 \\
\text { H } & -3.23366 & 3.05471 & -4.08877 \\
\text { H } & -1.64682 & 1.17152 & -3.82392 \\
\text { H } & -1.35506 & -2.56062 & 1.52746 \\
\text { H } & -3.22151 & -4.18616 & 1.59137 \\
\text { H } & -5.54274 & -3.43691 & 1.10848 \\
\text { H } & -5.97673 & -1.05230 & 0.56628 \\
\text { H } & -4.12280 & 0.56229 & 0.50143 \\
\text { O } & 0.55323 & 0.33447 & 2.10189 \\
\text { O } & -0.83737 & -0.02327 & 2.28719 \\
\text { H } & -2.33826 & 1.87625 & 1.50111 \\
\end{array}$ & & & & & & & & \\
\hline $\begin{array}{llll} & \end{array}$ & -2132.83813 & -2132.40891 & -2132.49655 & -2134.06012 & -2133.71854 & -2134.45709 & -2134.11551 & 1.8 \\
\hline
\end{tabular}




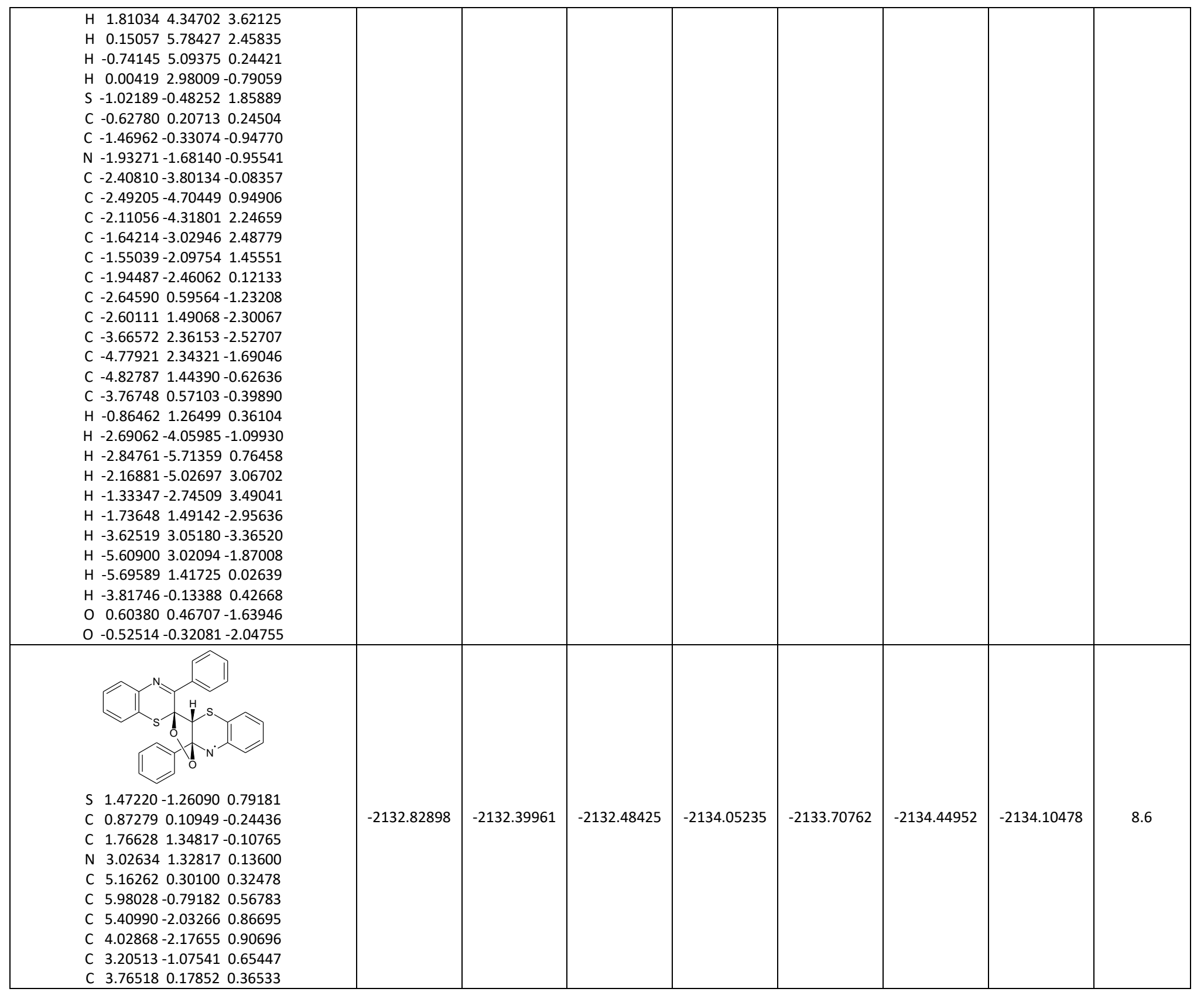




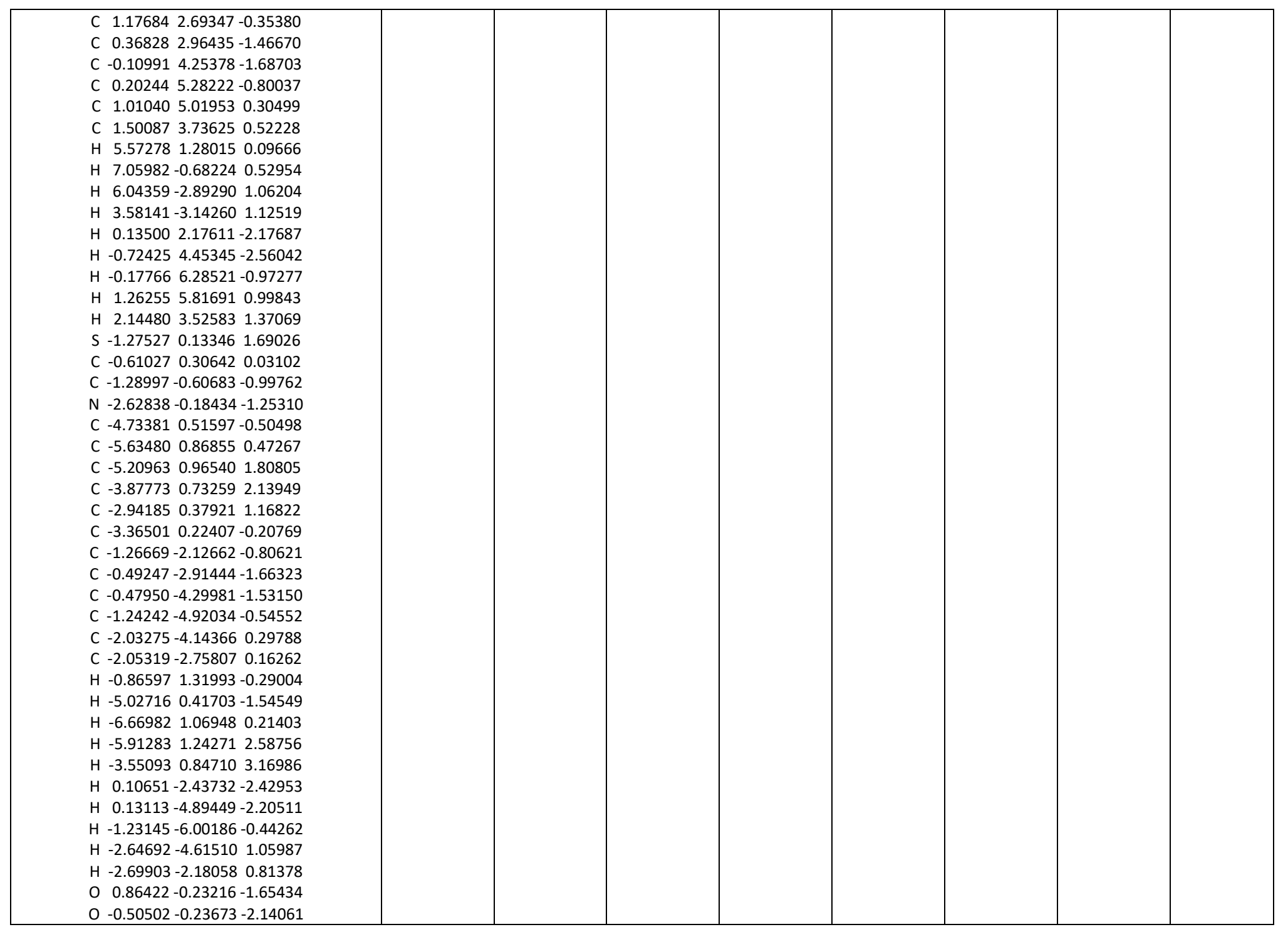




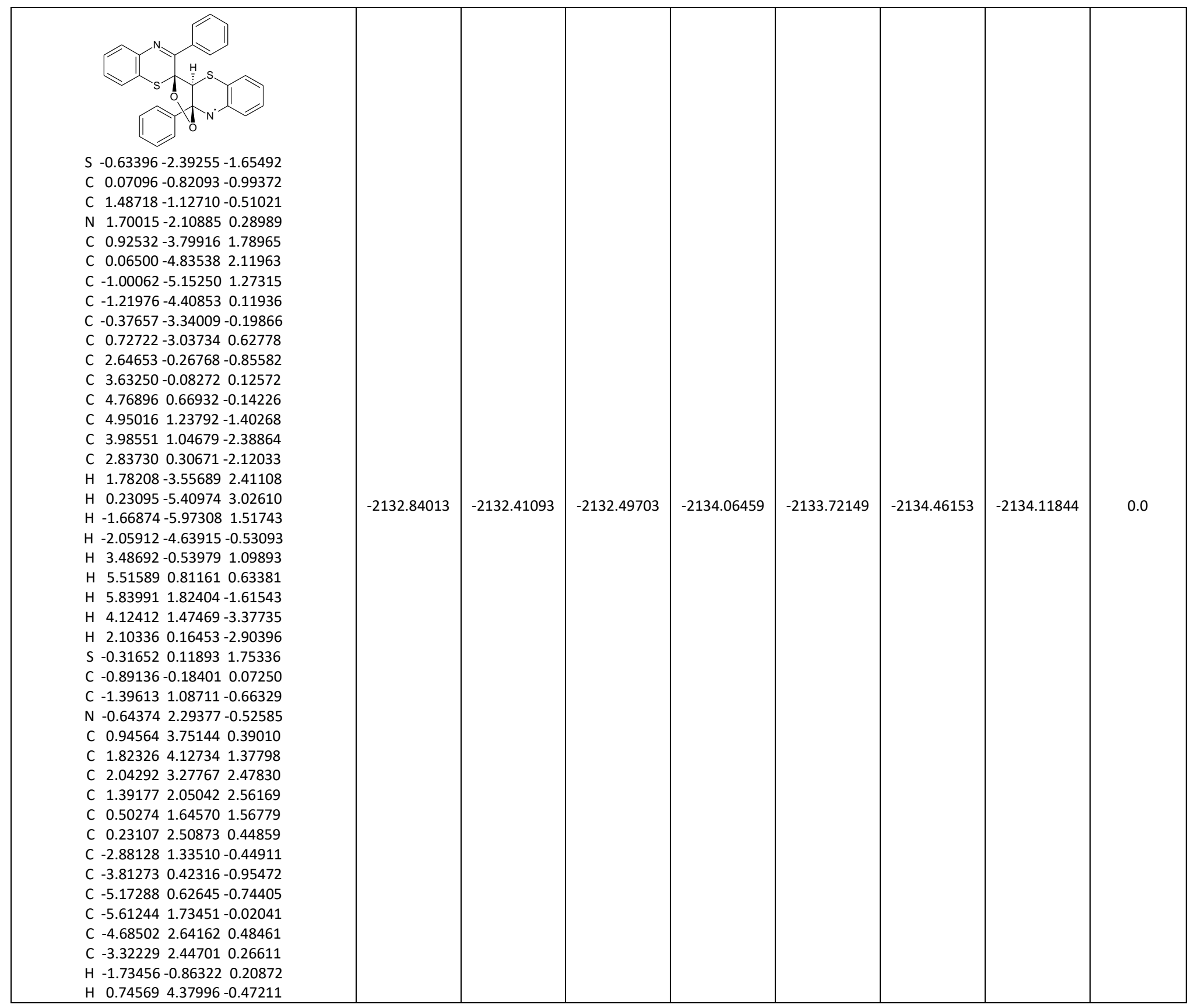




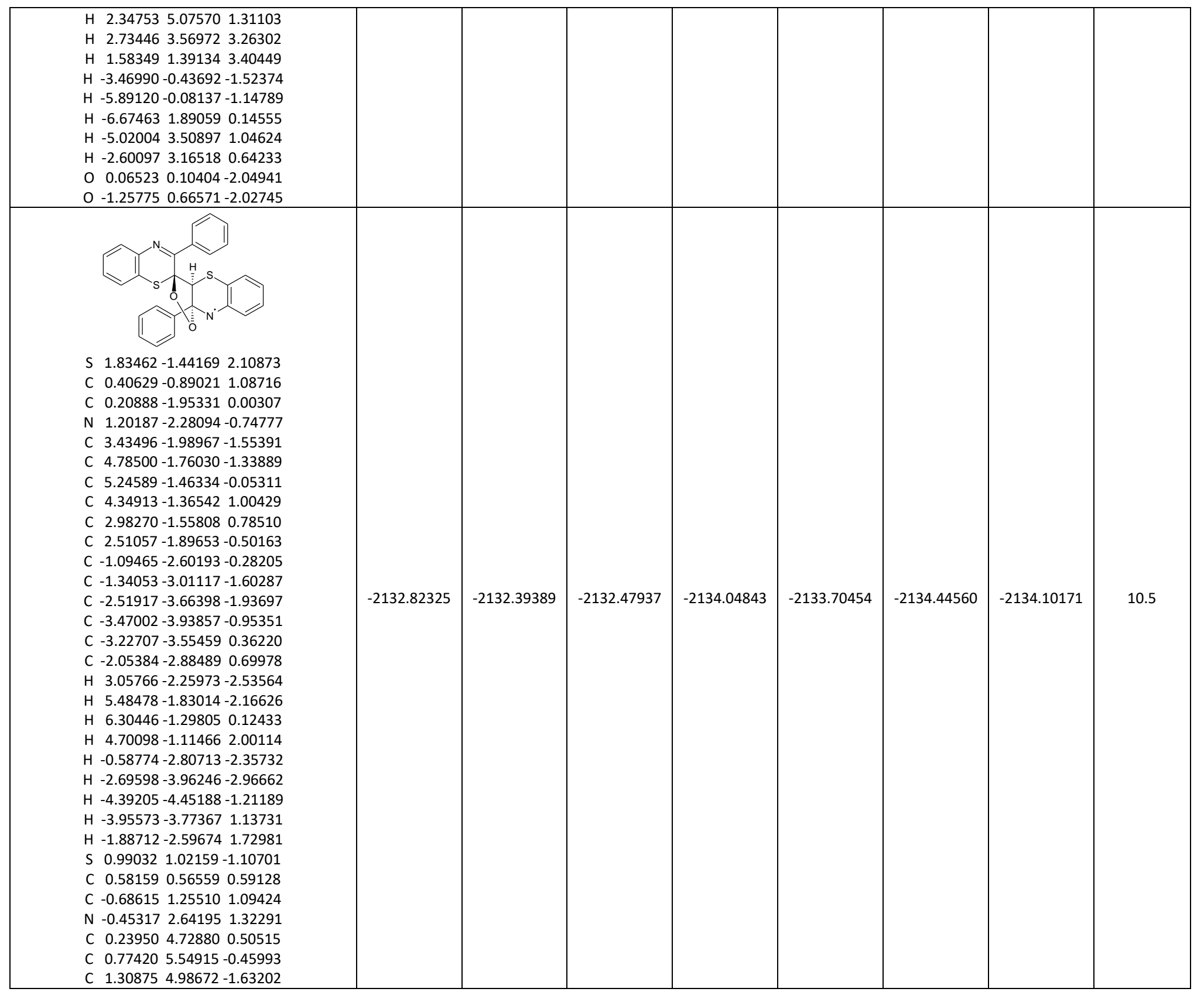




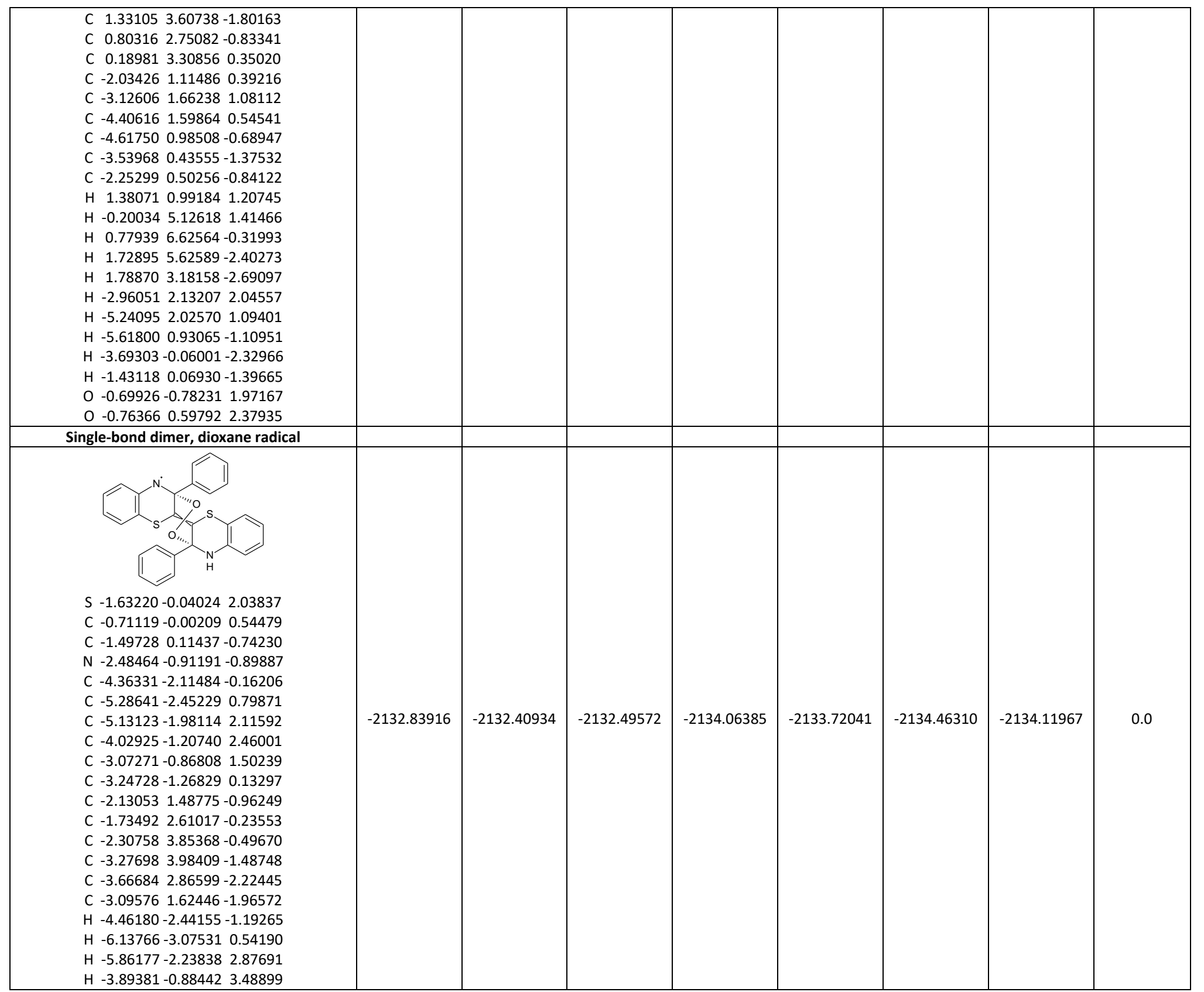




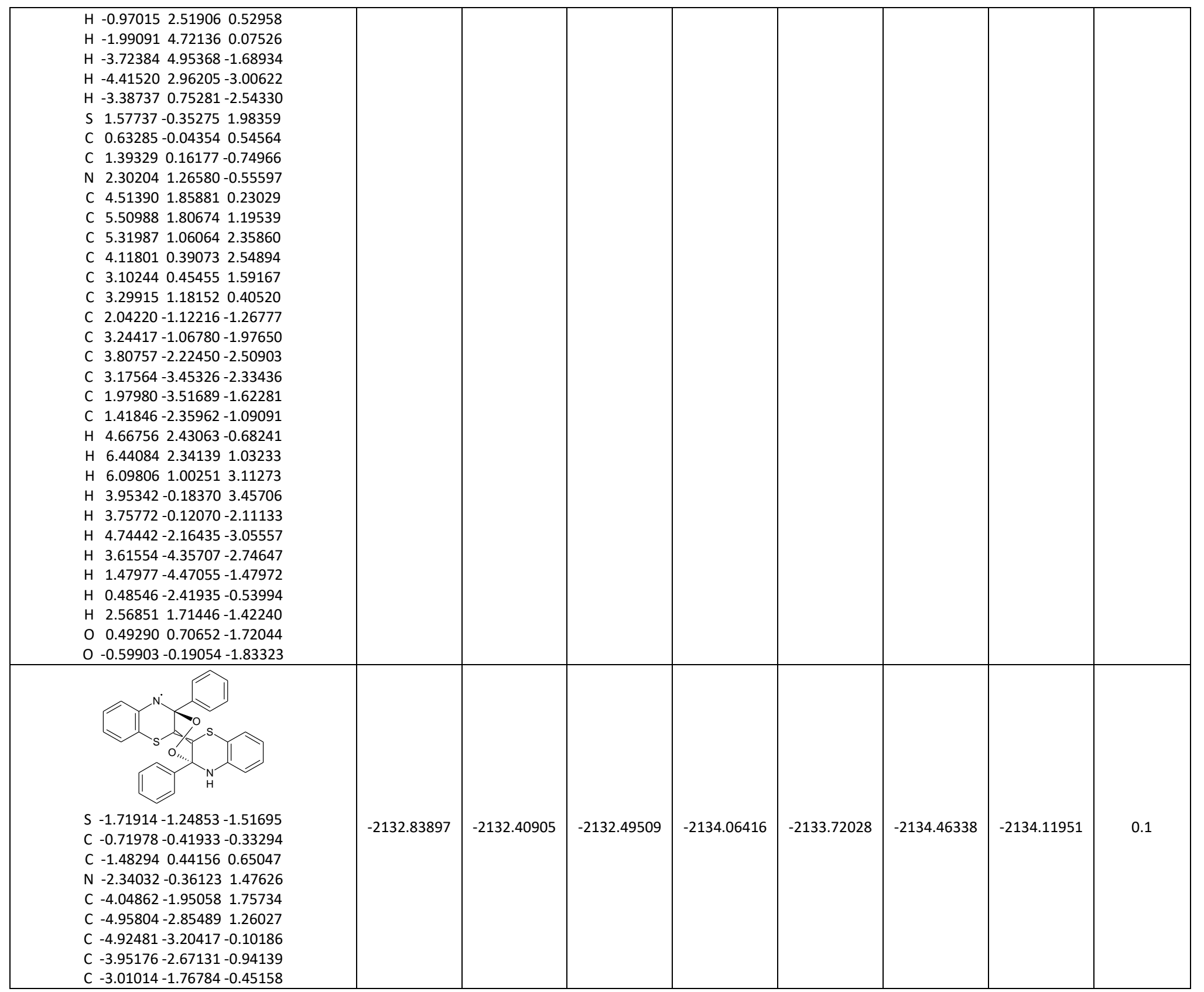




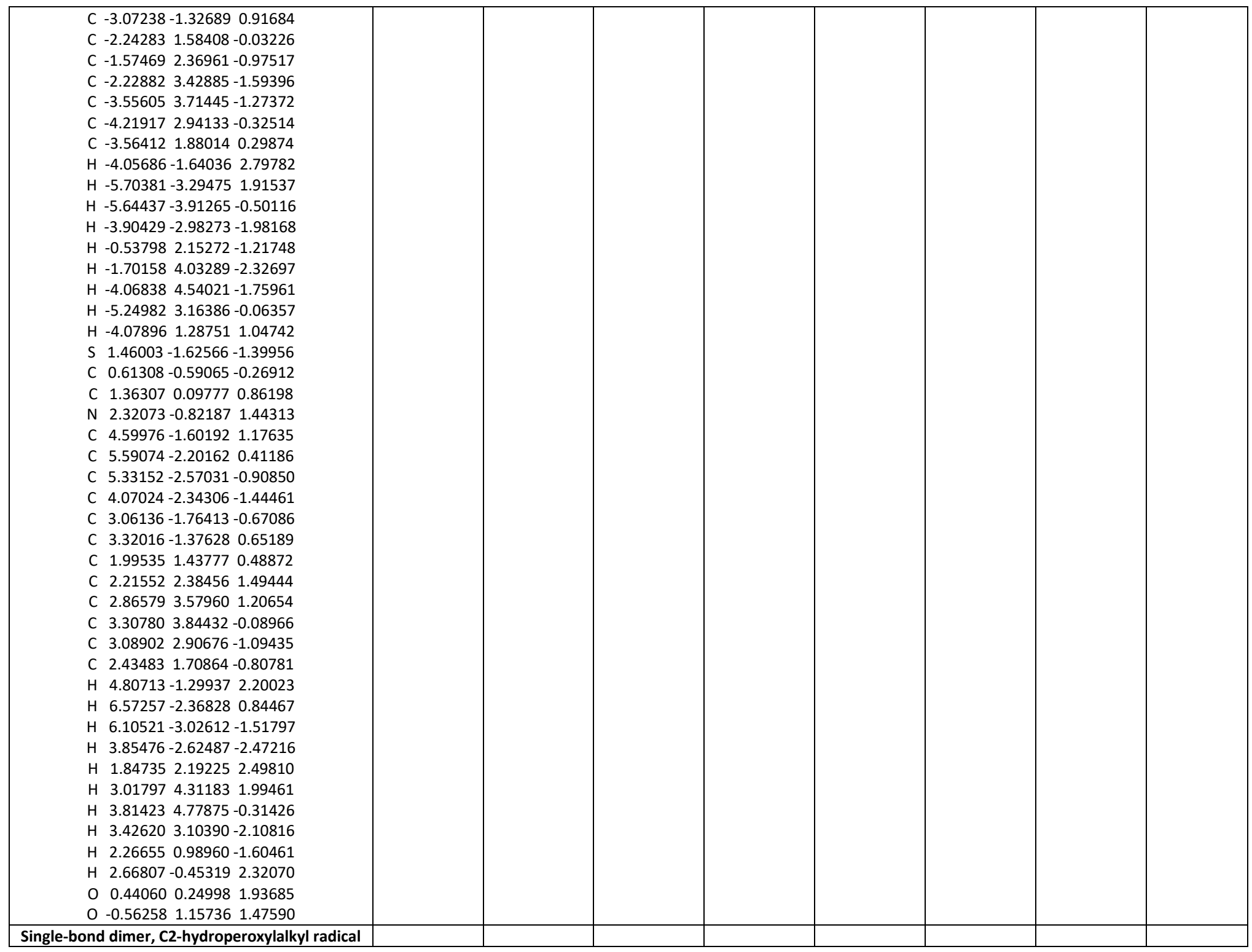




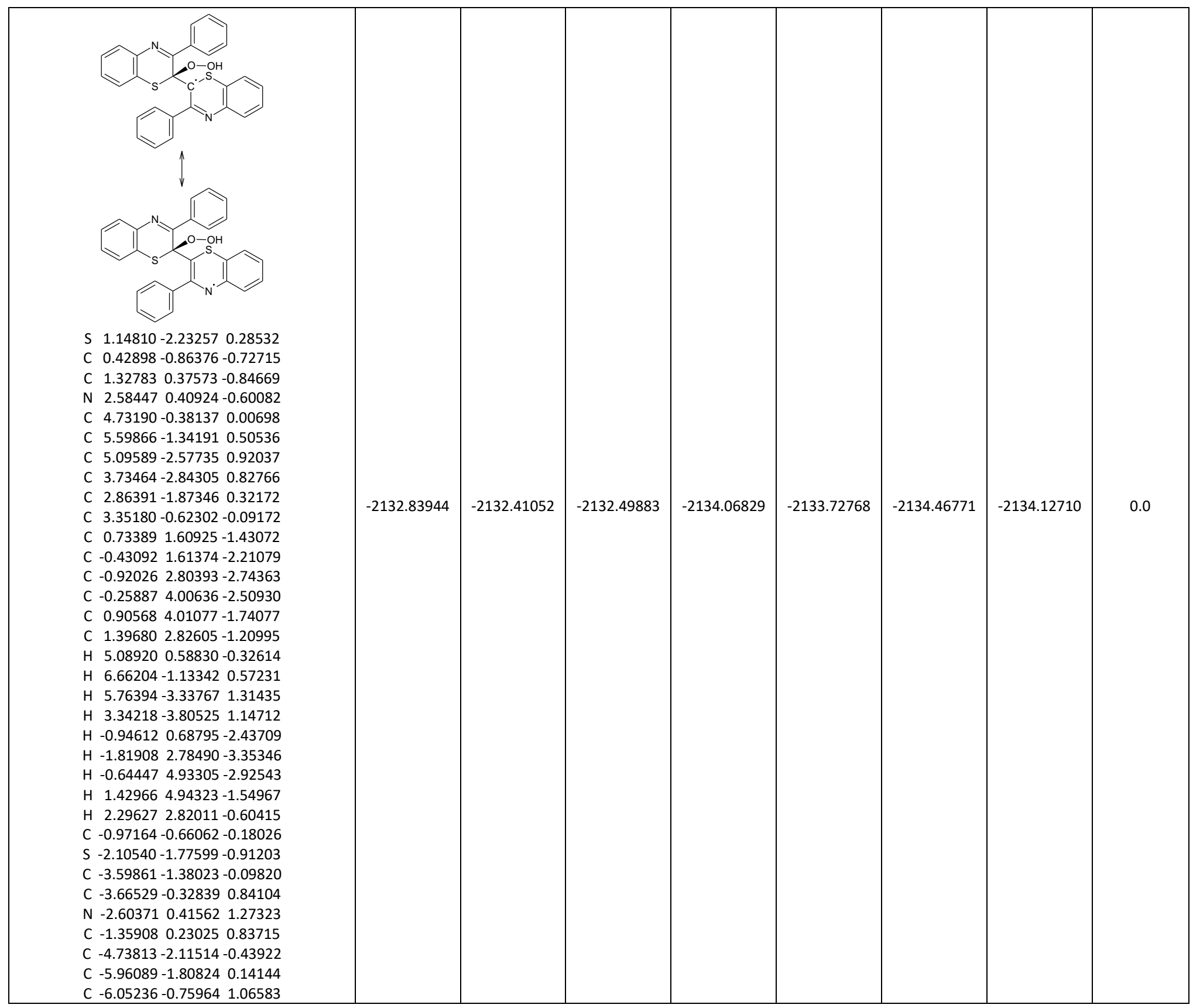




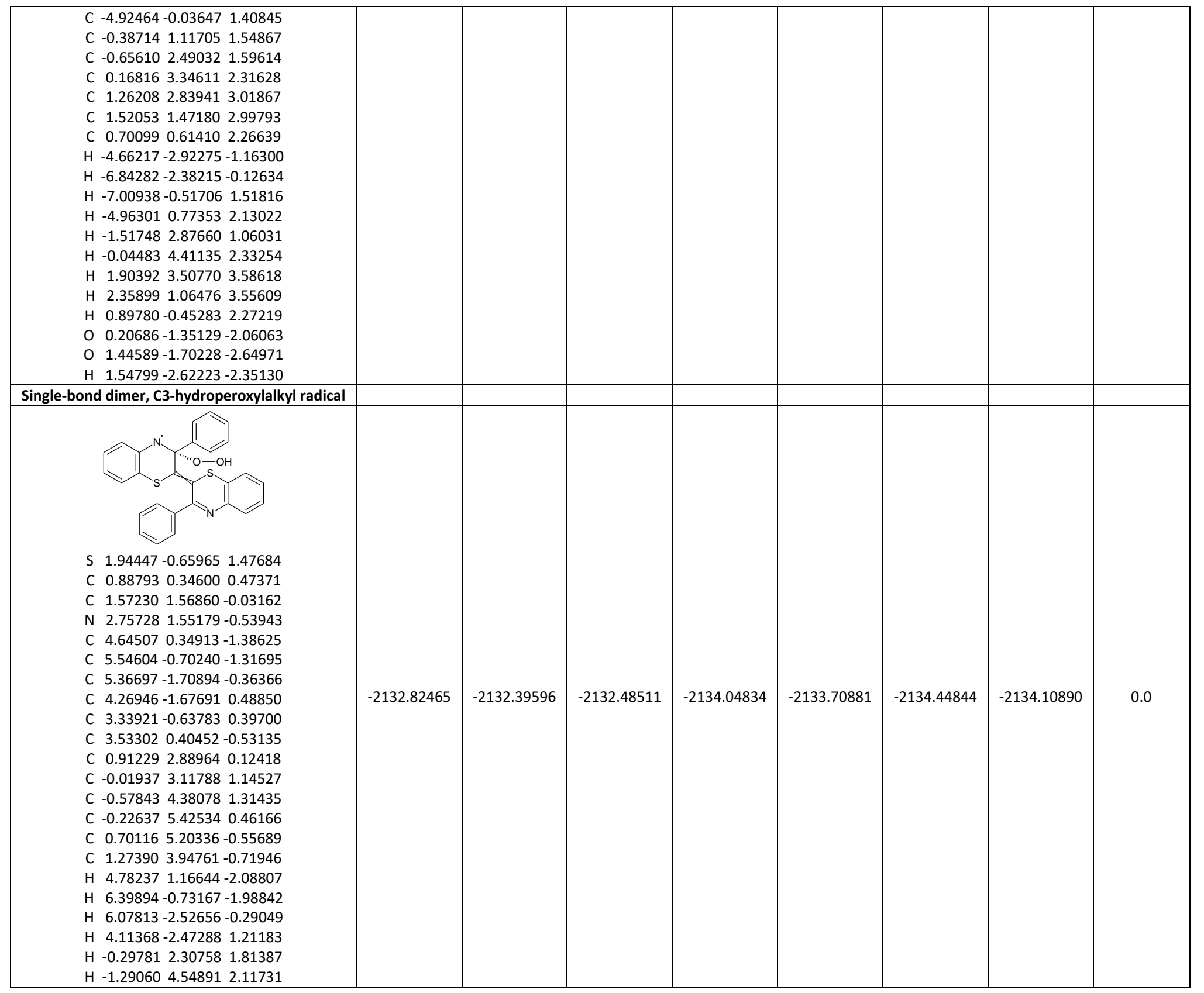




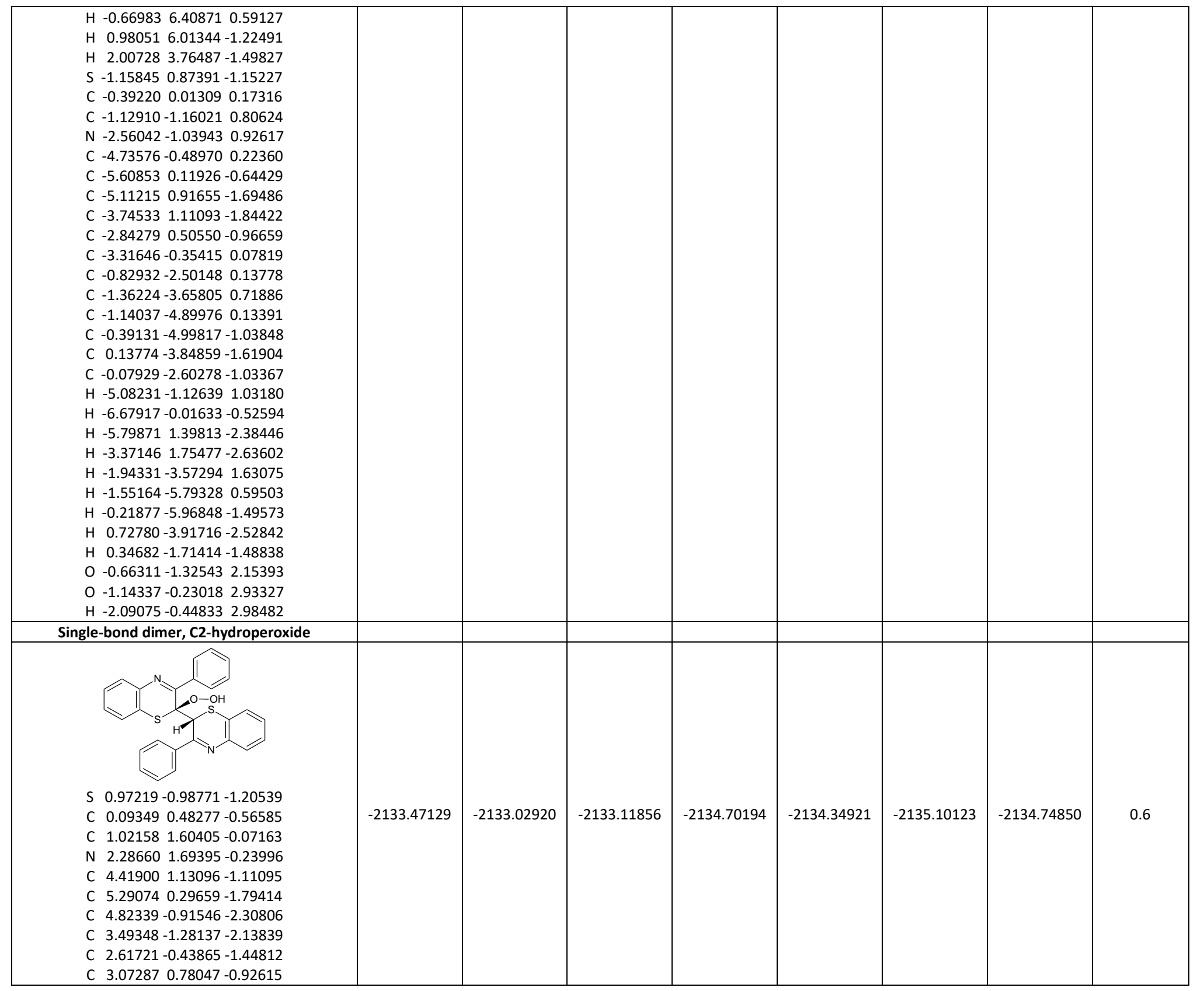




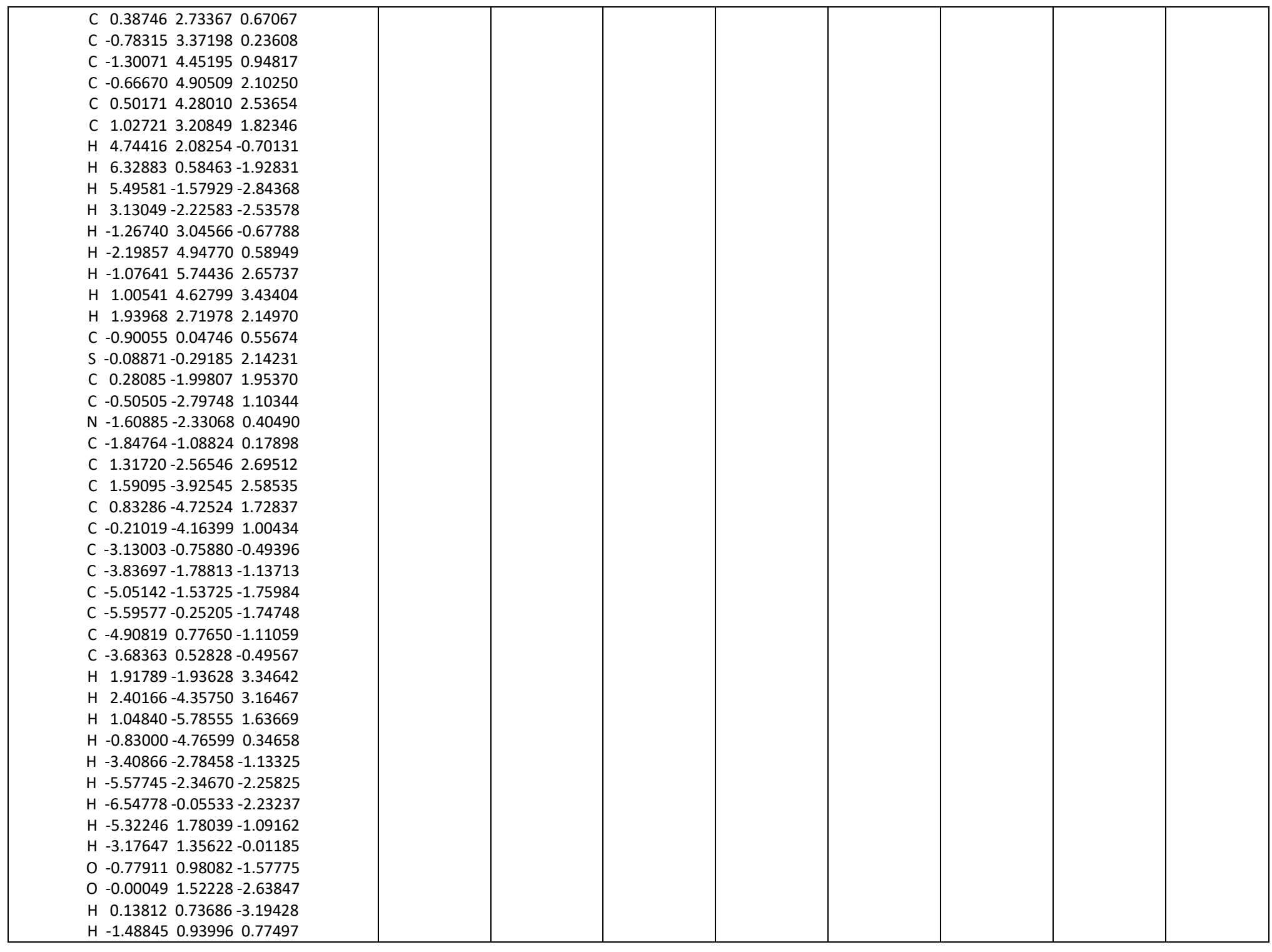




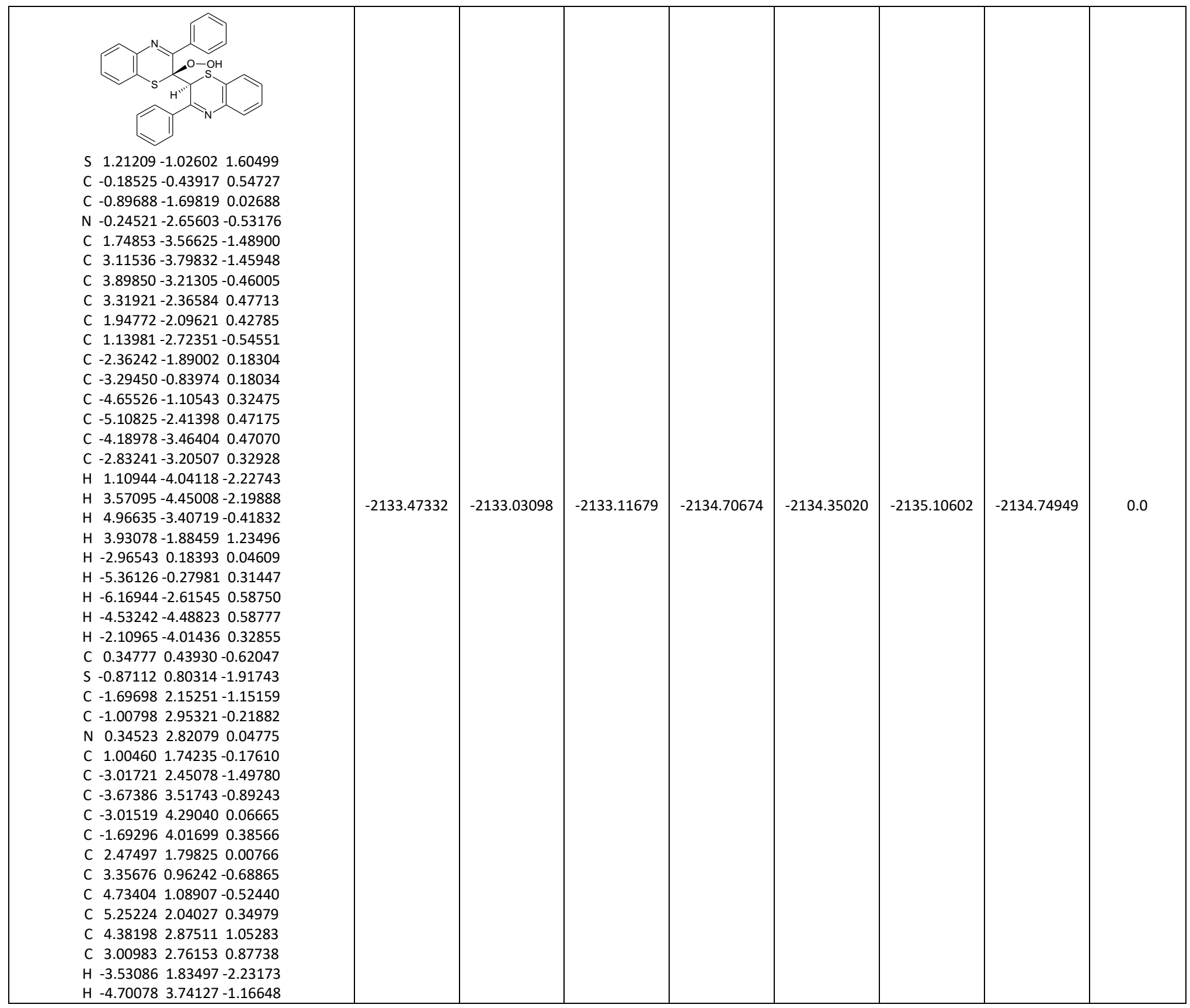




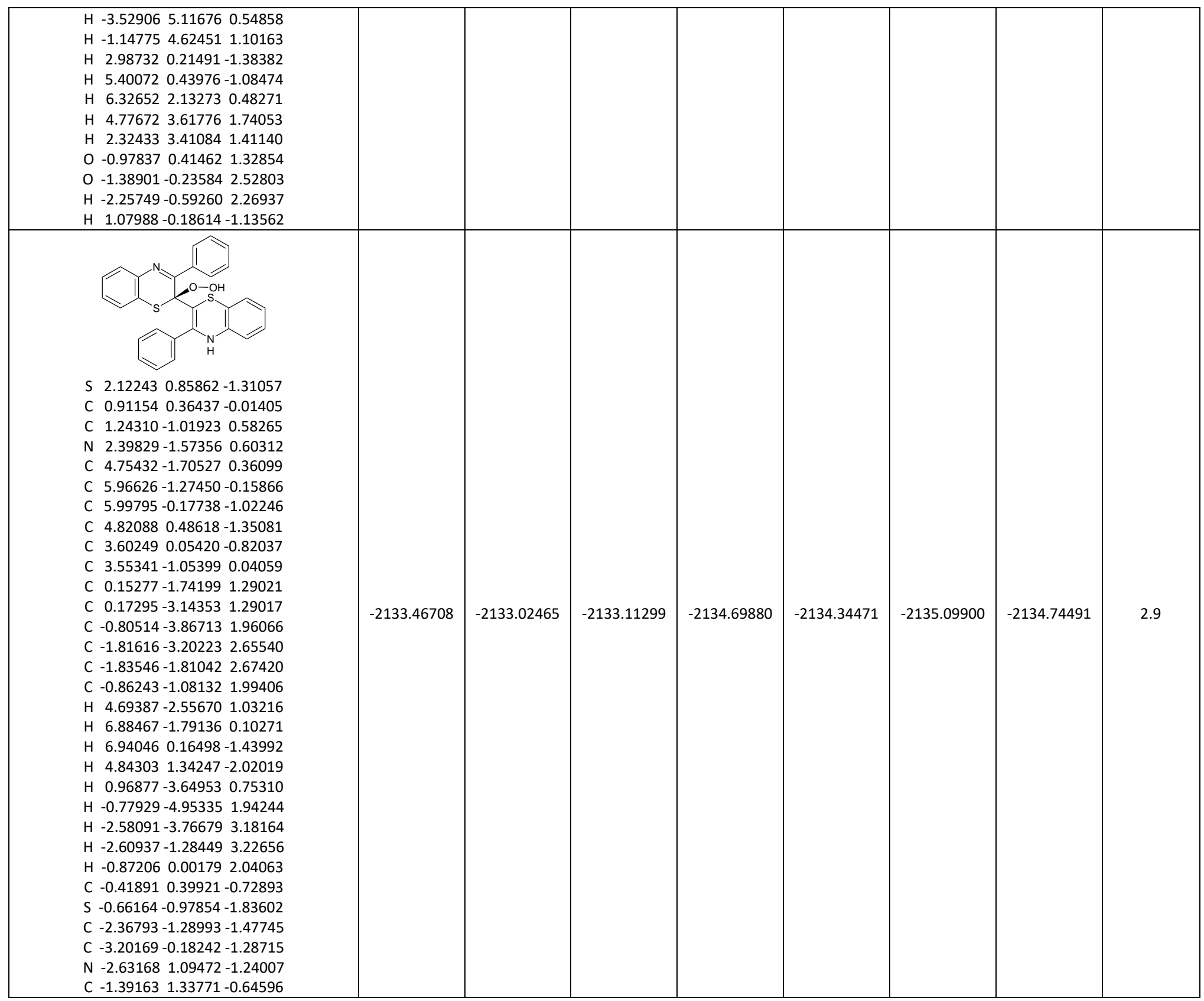




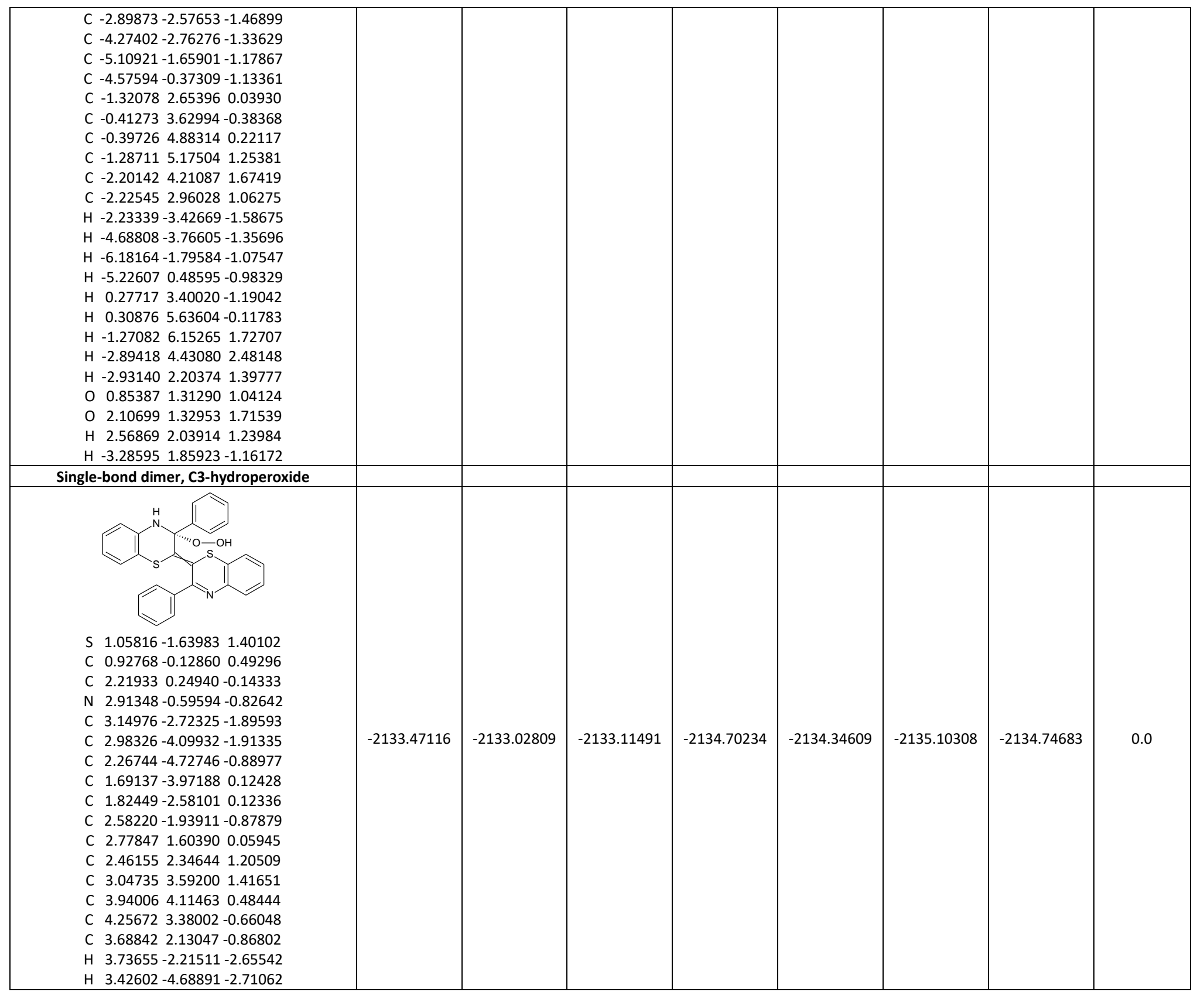




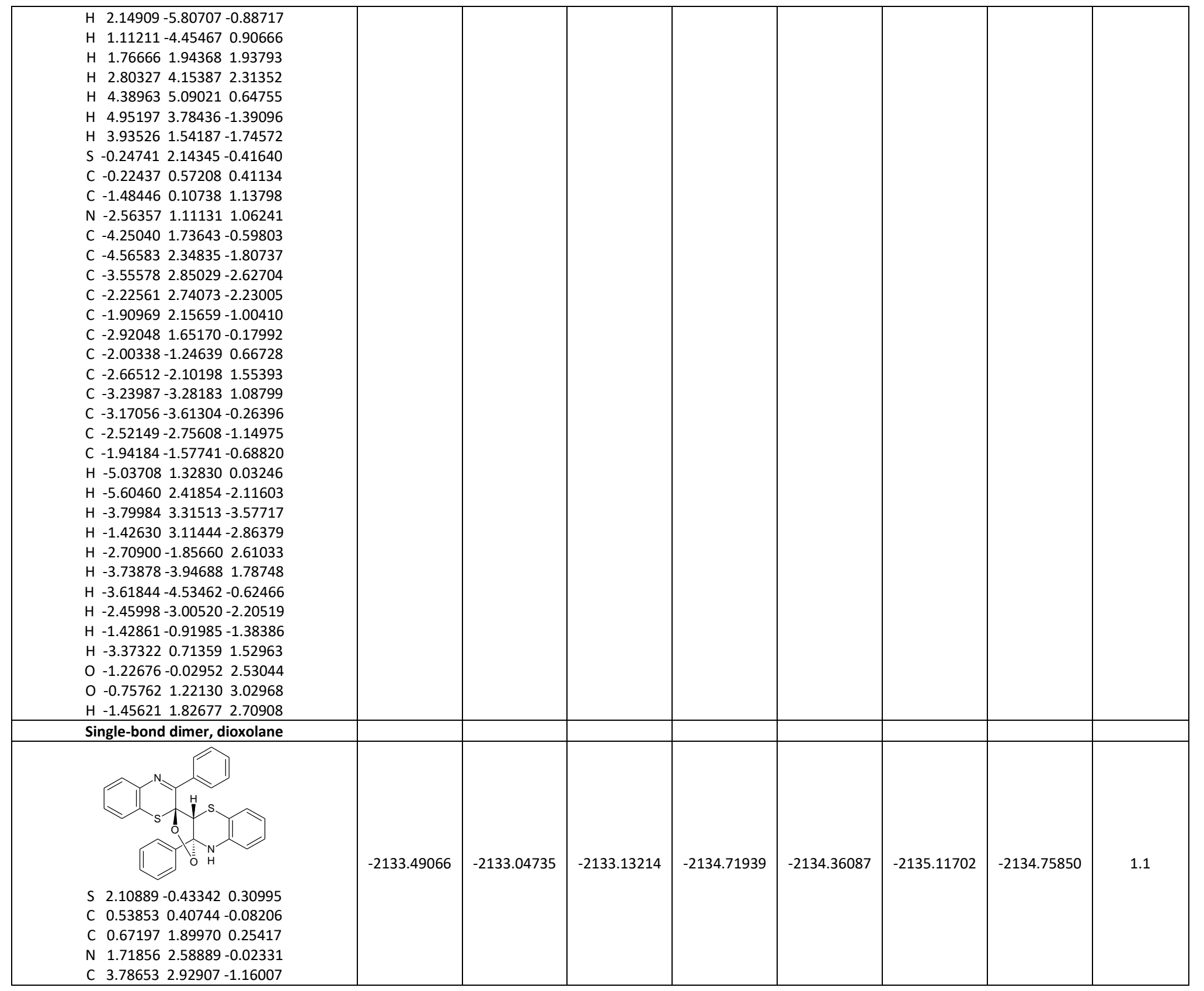




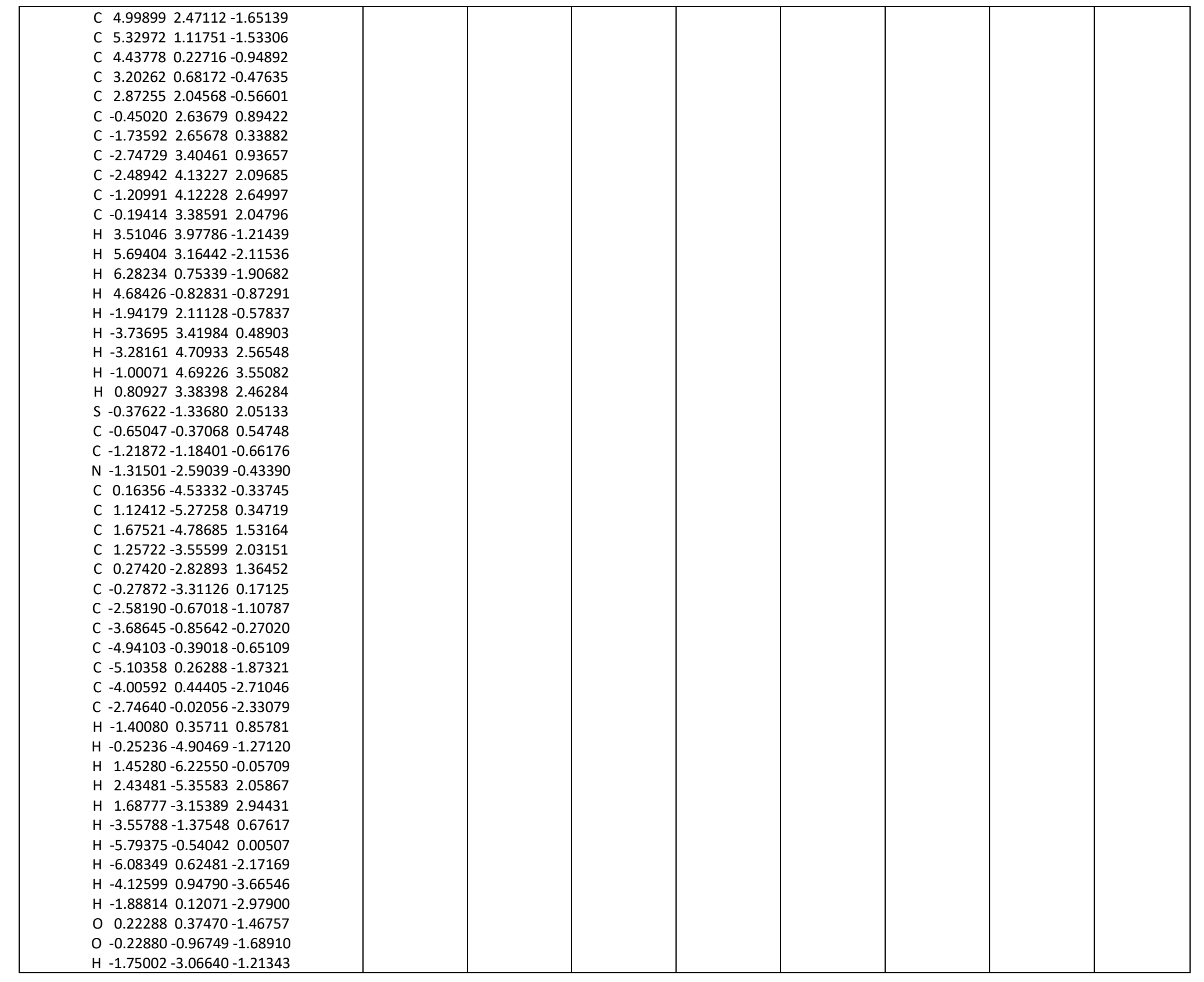




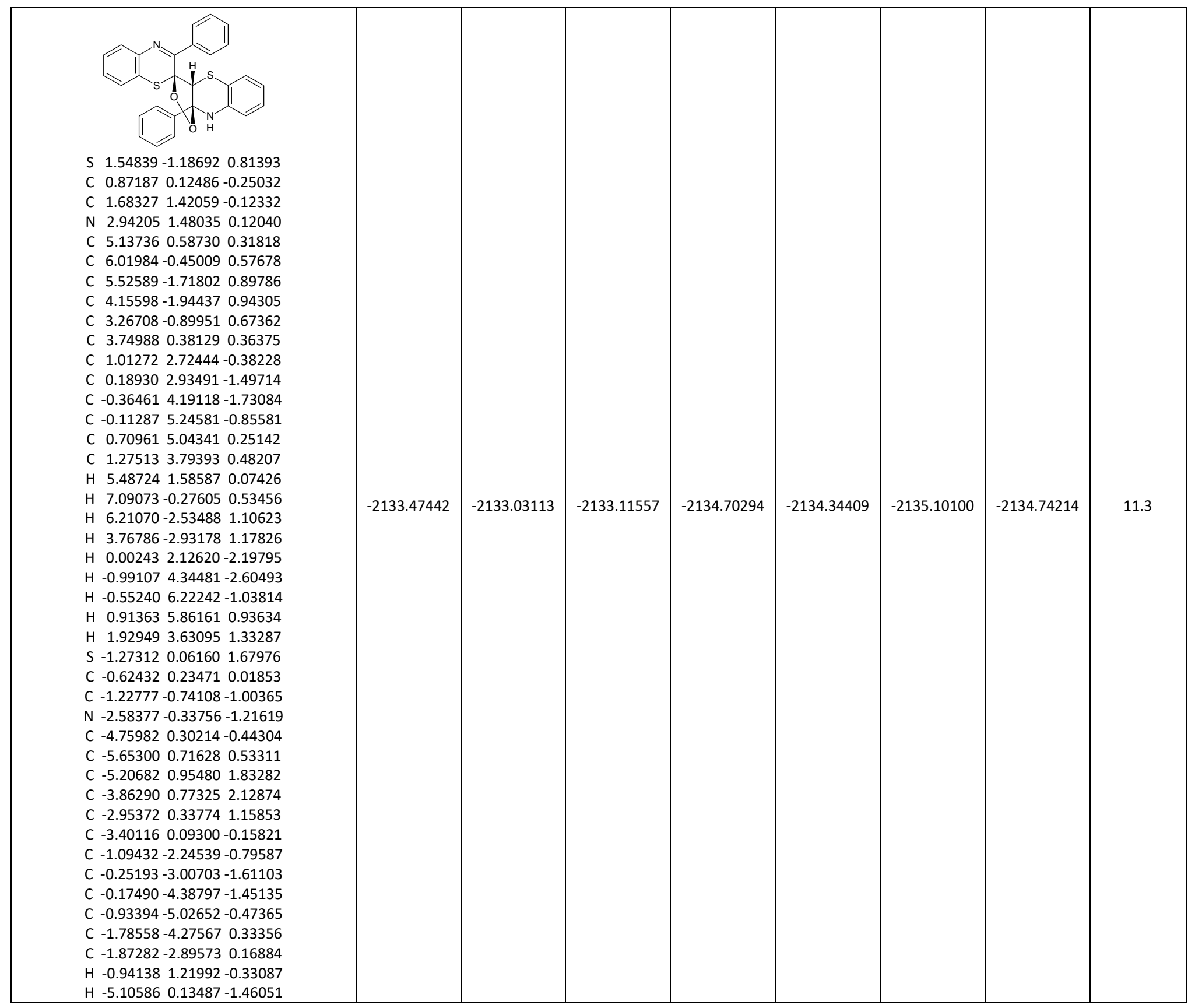




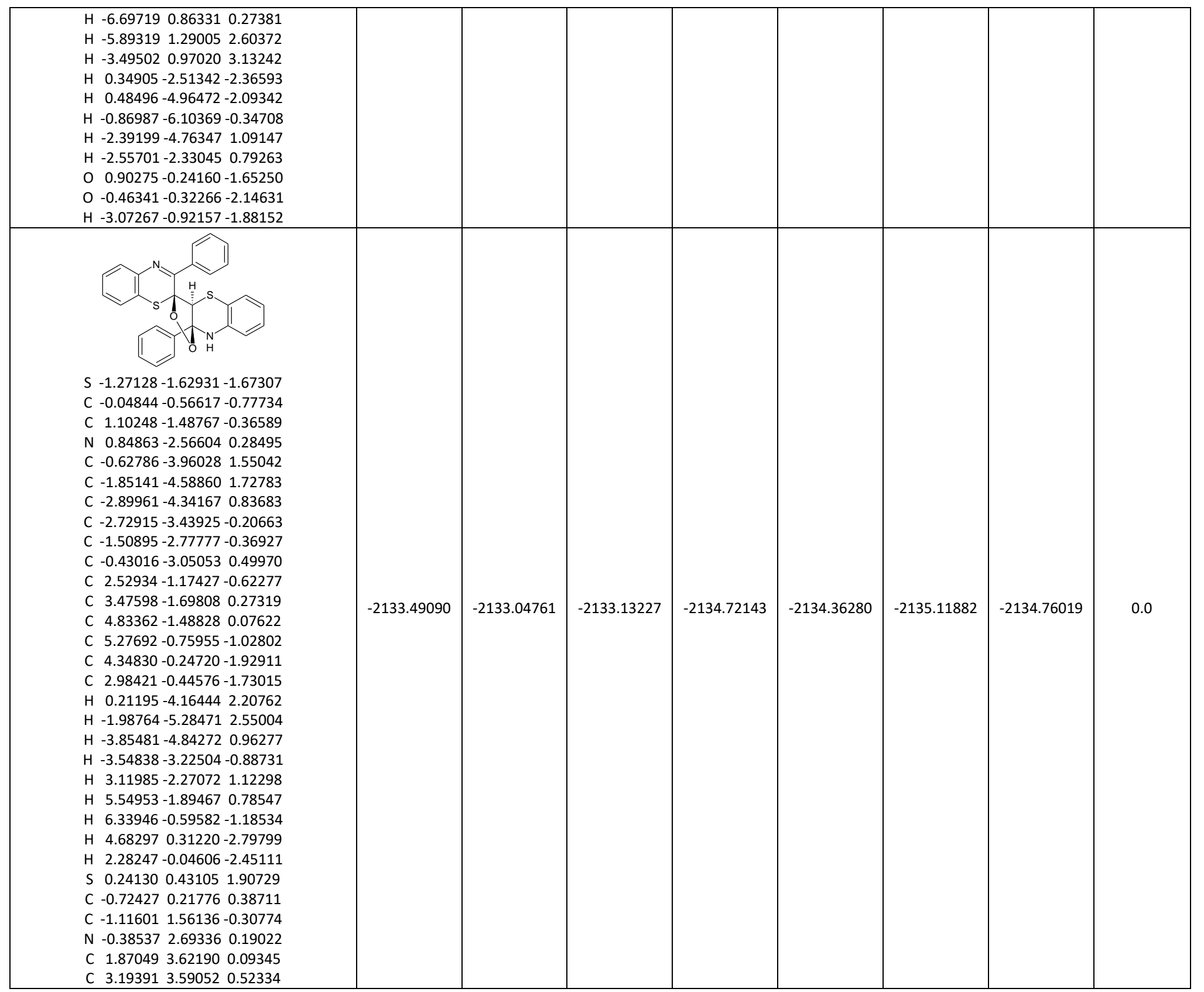




\begin{tabular}{|c|c|c|c|c|c|c|c|c|}
\hline $\begin{array}{lrlll}\text { C } & 3.64488 & 2.56040 & 1.34665 \\
\text { C } & 2.76217 & 1.55828 & 1.74221 \\
\text { C } & 1.43012 & 1.60339 & 1.33941 \\
\text { C } & 0.97322 & 2.63557 & 0.50934 \\
\text { C } & -2.60680 & 1.85945 & -0.24714 \\
\text { C } & -3.48507 & 1.36052 & -1.21349 \\
\text { C } & -4.84973 & 1.62151 & -1.11714 \\
\text { C } & -5.35148 & 2.36931 & -0.05391 \\
\text { C } & -4.47922 & 2.85891 & 0.91545 \\
\text { C } & -3.11209 & 2.60897 & 0.81916 \\
\text { H } & -1.62414 & -0.30580 & 0.71430 \\
\text { H } & 1.52451 & 4.41767 & -0.56234 \\
\text { H } & 3.87907 & 4.36836 & 0.19882 \\
\text { H } & 4.68128 & 2.52605 & 1.66749 \\
\text { H } & 3.10244 & 0.73738 & 2.36639 \\
\text { H } & -3.09757 & 0.76956 & -2.03563 \\
\text { H } & -5.52264 & 1.23750 & -1.87867 \\
\text { H } & -6.41687 & 2.56885 & 0.01901 \\
\text { H } & -4.86073 & 3.43876 & 1.75112 \\
\text { H } & -2.42794 & 2.98791 & 1.57182 \\
\text { O } & 0.33921 & 0.44577 & -1.66507 \\
\text { O } & -0.81334 & 1.30482 & -1.69656 \\
\text { H } & -0.63536 & 3.52532 & -0.33026 \\
\end{array}$ & & & & & & & & \\
\hline $\begin{array}{lrl} & \end{array}$ & -2133.46847 & -2133.02519 & -2133.10885 & -2134.70205 & -2134.34243 & -2135.10015 & -2134.74053 & 12.3 \\
\hline
\end{tabular}




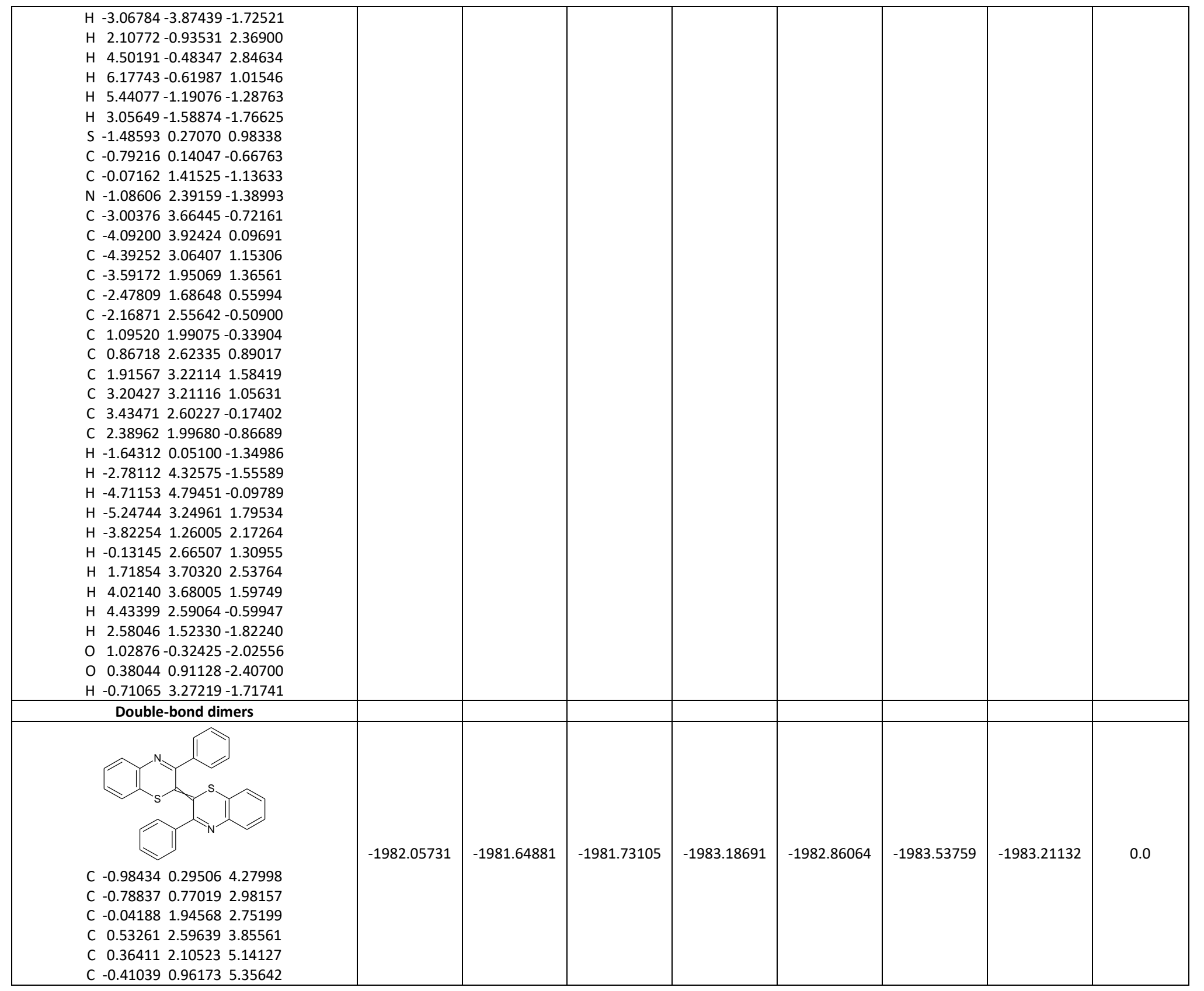




\begin{tabular}{|c|c|c|c|c|c|c|c|c|}
\hline $\begin{array}{lrrr}\text { N } & 0.03790 & 2.56192 & 1.51437 \\
\text { C } & -0.15152 & 1.91638 & 0.41273 \\
\text { C } & -0.32267 & 0.43894 & 0.40241 \\
\text { S } & -1.49413 & -0.09011 & 1.61496 \\
\text { C } & 0.32267 & -0.43894 & -0.40241 \\
\text { C } & 0.15152 & -1.91638 & -0.41273 \\
\text { N } & -0.03790 & -2.56192 & -1.51437 \\
\text { C } & 0.04188 & -1.94568 & -2.75199 \\
\text { C } & 0.78837 & -0.77019 & -2.98157 \\
\text { S } & 1.49413 & 0.09011 & -1.61496 \\
\text { C } & 0.98434 & -0.29506 & -4.27998 \\
\text { C } & 0.41039 & -0.96173 & -5.35642 \\
\text { C } & -0.36411 & -2.10523 & -5.14127 \\
\text { C } & -0.53261 & -2.59639 & -3.85561 \\
\text { C } & 0.26997 & -2.70456 & 0.83582 \\
\text { C } & -0.26997 & 2.70456 & -0.83582 \\
\text { H } & 1.09834 & 3.50392 & 3.66718 \\
\text { H } & 0.81969 & 2.62068 & 5.98147 \\
\text { H } & -0.55970 & 0.58278 & 6.36318 \\
\text { H } & -1.56371 & -0.61020 & 4.43871 \\
\text { H } & -1.09834 & -3.50392 & -3.66718 \\
\text { H } & -0.81969 & -2.62068 & -5.98147 \\
\text { H } & 0.55970 & -0.58278 & -6.36318 \\
\text { H } & 1.56371 & 0.61020 & -4.43871 \\
\text { C } & 1.03885 & -2.24432 & 1.91124 \\
\text { C } & 1.19810 & -3.02795 & 3.04944 \\
\text { C } & 0.58124 & -4.27506 & 3.13252 \\
\text { C } & -0.18791 & -4.73908 & 2.06485 \\
\text { C } & -0.33607 & -3.96429 & 0.92061 \\
\text { H } & 1.52168 & -1.27294 & 1.85326 \\
\text { H } & 1.80509 & -2.66204 & 3.87261 \\
\text { H } & 0.70076 & -4.88423 & 4.02416 \\
\text { H } & -0.67210 & -5.70983 & 2.12434 \\
\text { H } & -0.92121 & -4.31860 & 0.07824 \\
\text { C } & 0.33607 & 3.96429 & -0.92061 \\
\text { C } & 0.18791 & 4.73908 & -2.06485 \\
\text { C } & -0.58124 & 4.27506 & -3.13252 \\
\text { C } & -1.19810 & 3.02795 & -3.04944 \\
\text { C } & -1.03885 & 2.24432 & -1.91124 \\
\text { H } & 0.92121 & 4.31860 & -0.07824 \\
\text { H } & 0.67210 & 5.70983 & -2.12434 \\
\text { H } & -0.70076 & 4.88423 & -4.02416 \\
\text { H } & -1.80509 & 2.66204 & -3.87261 \\
\text { H } & -1.52168 & 1.27294 & -1.85326\end{array}$ & & & & & & & & \\
\hline \multicolumn{9}{|l|}{ Oxygen species } \\
\hline \begin{tabular}{cccc} 
& \multicolumn{3}{c}{$\mathrm{O}_{2}$} \\
$\mathrm{O}$ & 0.00000 & 0.00000 & 0.60144 \\
$\mathrm{O}$ & 0.00000 & 0.00000 & -0.60144 \\
\end{tabular} & -150.17434 & -150.16711 & -150.19038 & -150.26391 & -150.27995 & -150.31368 & -150.32972 & 0.0 \\
\hline $\begin{array}{ccc} & \mathrm{H}_{2} \mathrm{O}_{2} \\
\mathrm{O} & 0.00000 & 0.71589-0.05491\end{array}$ & -151.39619 & -151.36500 & -151.39074 & -151.48905 & -151.48360 & -151.54187 & -151.53642 & 0.0 \\
\hline
\end{tabular}




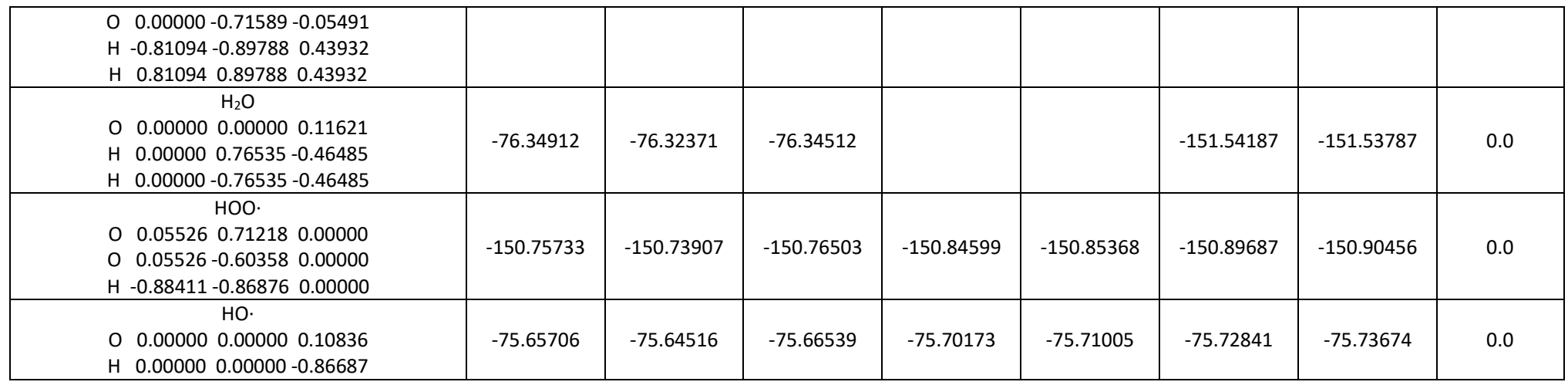


Table S4. Neutral forms in methanol. For each chemical species, values are reported for the most stable conformer / geometric isomer identified. $G_{\text {RRHO,calc }}[M 062 X / 6-311++G(2 d, 2 p) / S M D]=G_{\text {el }}[M 062 X / 6-311++G(2 d, 2 p) / S M D / / P B E 0 / 6-31+G(d, p) / P C M]-G_{\text {el }}[P B E 0 / 6-31+G(d, p) / P C M]+$

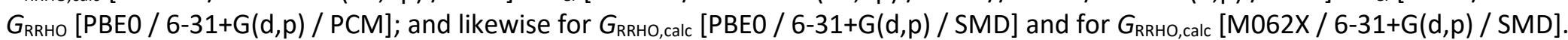

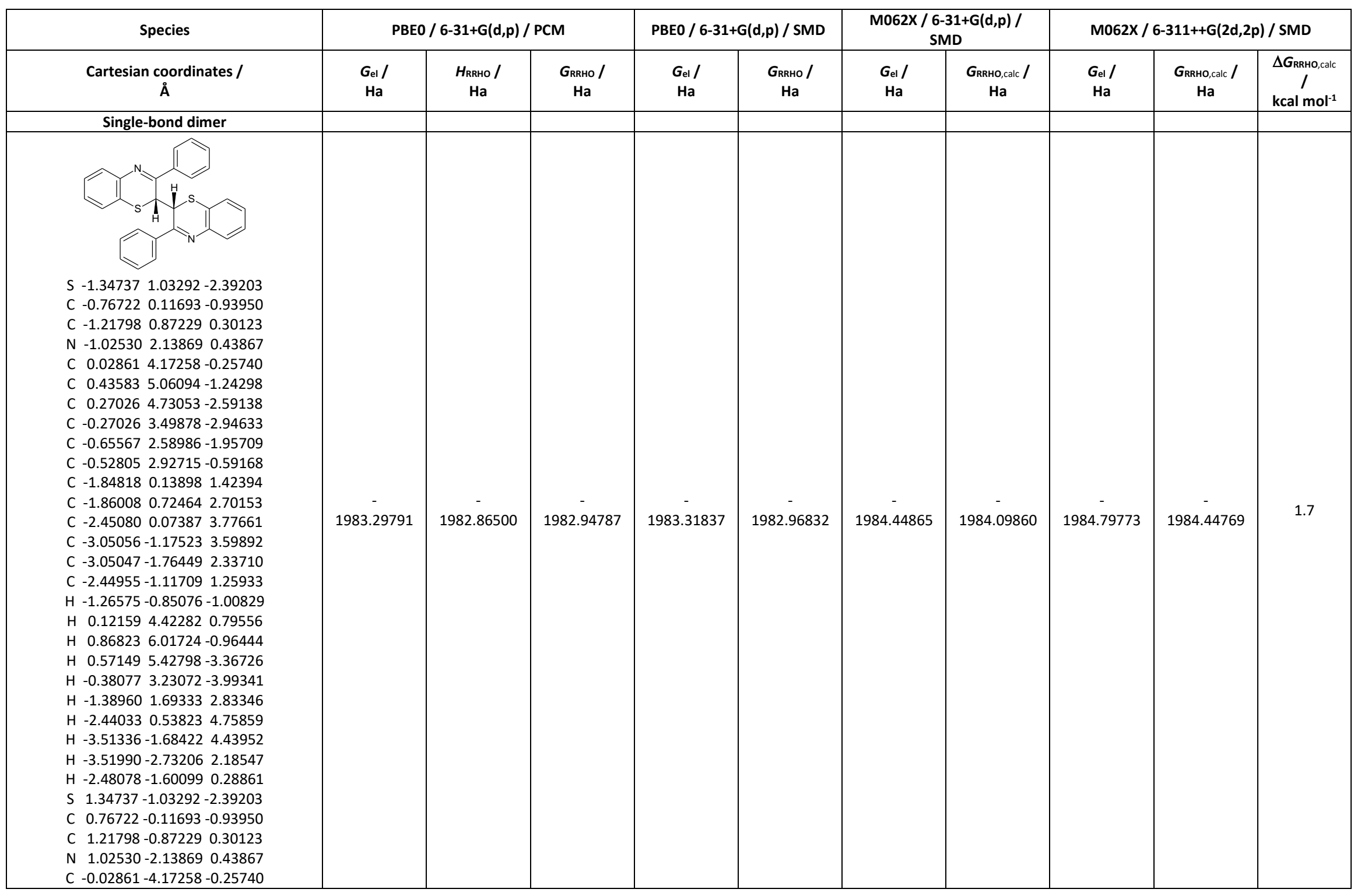




\begin{tabular}{|c|c|c|c|c|c|c|c|c|c|c|}
\hline $\begin{array}{llll}\text { C } & -0.43583 & -5.06094 & -1.24298 \\
\text { C } & -0.27026 & -4.73053 & -2.59138 \\
\text { C } & 0.27026 & -3.49878 & -2.94633 \\
\text { C } & 0.65567 & -2.58986 & -1.95709 \\
\text { C } & 0.52805 & -2.92715 & -0.59168 \\
\text { C } & 1.84818 & -0.13898 & 1.42394 \\
\text { C } & 1.86008 & -0.72464 & 2.70153 \\
\text { C } & 2.45080 & -0.07387 & 3.77661 \\
\text { C } & 3.05056 & 1.17523 & 3.59892 \\
\text { C } & 3.05047 & 1.76449 & 2.33710 \\
\text { C } & 2.44955 & 1.11709 & 1.25933 \\
\text { H } & 1.26575 & 0.85076 & -1.00829 \\
\text { H } & -0.12159 & -4.42282 & 0.79556 \\
\text { H } & -0.86823 & -6.01724 & -0.96444 \\
\text { H } & -0.57149 & -5.42798 & -3.36726 \\
\text { H } & 0.38077 & -3.23072 & -3.99341 \\
\text { H } & 1.38960 & -1.69333 & 2.83346 \\
\text { H } & 2.44033 & -0.53823 & 4.75859 \\
\text { H } & 3.51336 & 1.68422 & 4.43952 \\
\text { H } & 3.51990 & 2.73206 & 2.18547 \\
\text { H } & 2.48078 & 1.60099 & 0.28861 \\
\end{array}$ & & & & & & & & & & \\
\hline $\begin{array}{llll}\text { S } & 1.00027 & -0.75723 & -1.84385 \\
\mathrm{C} & -0.28417 & -0.36409 & -0.61977 \\
\mathrm{C} & -0.97808 & -1.66332 & -0.24538 \\
\mathrm{~N} & -0.31501 & -2.70859 & 0.11063 \\
\mathrm{C} & 1.74075 & -3.75167 & 0.76503 \\
\mathrm{C} & 3.10284 & -3.96606 & 0.60781 \\
\mathrm{C} & 3.82226 & -3.22229 & -0.33270 \\
\mathrm{C} & 3.18225 & -2.24109 & -1.08221 \\
\mathrm{C} & 1.81735 & -1.99979 & -0.90410 \\
\mathrm{C} & 1.07044 & -2.77447 & 0.01103 \\
\mathrm{C} & -2.45647 & -1.74638 & -0.25337 \\
\mathrm{C} & -3.09118 & -2.73139 & 0.52202 \\
\mathrm{C} & -4.47459 & -2.85275 & 0.52213 \\
\mathrm{C} & -5.25496 & -2.00107 & -0.26376 \\
\mathrm{C} & -4.63712 & -1.02649 & -1.04339 \\
\mathrm{C} & -3.24988 & -0.89318 & -1.03301 \\
\mathrm{H} & -0.96460 & 0.30970 & -1.14221 \\
\mathrm{H} & 1.15801 & -4.34297 & 1.46566 \\
\mathrm{H} & 3.60353 & -4.72340 & 1.20351 \\
\mathrm{H} & 4.88445 & -3.39833 & -0.47411 \\
\mathrm{H} & 3.74341 & -1.64413 & -1.79574 \\
\mathrm{H} & -2.47911 & -3.39044 & 1.12892\end{array}$ & $\begin{array}{l}- \\
- \\
-1983.29751\end{array}$ & $\begin{array}{c}- \\
1982.86470\end{array}$ & (1) & $\begin{array}{c}- \\
1983.31826\end{array}$ & 1982.96991 & $\begin{array}{c}- \\
- \\
1984.44902\end{array}$ & $\begin{array}{c}- \\
- \\
1984.10067\end{array}$ & $\begin{array}{c} \\
\\
- \\
- \\
1984.79871\end{array}$ & $\stackrel{-}{-}$ & 0.0 \\
\hline
\end{tabular}




\begin{tabular}{|c|c|c|c|c|c|c|c|c|c|c|}
\hline $\begin{array}{llll}\text { H } & -4.94843 & -3.61177 & 1.13812 \\
\text { H } & -6.33686 & -2.09775 & -0.26610 \\
\text { H } & -5.23341 & -0.36374 & -1.66372 \\
\text { H } & -2.79982 & -0.13185 & -1.66208 \\
\text { S } & -1.00027 & 0.75723 & 1.84385 \\
\text { C } & 0.28417 & 0.36409 & 0.61977 \\
\text { C } & 0.97808 & 1.66332 & 0.24538 \\
\text { N } & 0.31501 & 2.70859 & -0.11063 \\
\text { C } & -1.74075 & 3.75167 & -0.76503 \\
\text { C } & -3.10284 & 3.96606 & -0.60781 \\
\text { C } & -3.82226 & 3.22229 & 0.33270 \\
\text { C } & -3.18225 & 2.24109 & 1.08221 \\
\text { C } & -1.81735 & 1.99979 & 0.90410 \\
\text { C } & -1.07044 & 2.77447 & -0.01103 \\
\text { C } & 2.45647 & 1.74638 & 0.25337 \\
\text { C } & 3.09118 & 2.73139 & -0.52202 \\
\text { C } & 4.47459 & 2.85275 & -0.52213 \\
\text { C } & 5.25496 & 2.00107 & 0.26376 \\
\text { C } & 4.63712 & 1.02649 & 1.04339 \\
\text { C } & 3.24988 & 0.89318 & 1.03301 \\
\text { H } & 0.96460 & -0.30970 & 1.14221 \\
\text { H } & -1.15801 & 4.34297 & -1.46566 \\
\text { H } & -3.60353 & 4.72340 & -1.20351 \\
\text { H } & -4.88445 & 3.39833 & 0.47411 \\
\text { H } & -3.74341 & 1.64413 & 1.79574 \\
\text { H } & 2.47911 & 3.39044 & -1.12892 \\
\text { H } & 4.94843 & 3.61177 & -1.13812 \\
\text { H } & 6.33686 & 2.09775 & 0.26610 \\
\text { H } & 5.23341 & 0.36374 & 1.66372 \\
\text { H } & 2.79982 & 0.13185 & 1.66208 \\
\end{array}$ & & & & & & & & & & \\
\hline $\begin{array}{llll}\mathrm{S} & -1.66149 & -2.01085 & -1.34836 \\
\mathrm{C} & -0.46001 & -1.13796 & -0.26838 \\
\mathrm{C} & -1.07181 & -0.92777 & 1.11193 \\
\mathrm{~N} & -2.30503 & -0.62886 & 1.32189 \\
\mathrm{C} & -4.44042 & 0.15876 & 0.61134 \\
\mathrm{C} & -5.46391 & 0.26499 & -0.32137 \\
\mathrm{C} & -5.32233 & -0.32902 & -1.57836 \\
\mathrm{C} & -4.15016 & -1.00634 & -1.90085 \\
\mathrm{C} & -3.11333 & -1.10038 & -0.96858 \\
\mathrm{C} & -3.25150 & -0.52301 & 0.30951 \\
\mathrm{C} & -0.17371 & -1.00136 & 2.29388 \\
\mathrm{C} & -0.63928 & -0.53127 & 3.53436 \\
\mathrm{C} & 0.16228 & -0.58742 & 4.66705\end{array}$ & $\begin{array}{c}- \\
- \\
- \\
1983.29263\end{array}$ & $\begin{array}{l}- \\
- \\
1982.85921\end{array}$ & $\begin{array}{c}- \\
- \\
1982.94366\end{array}$ & 1983.31313 & 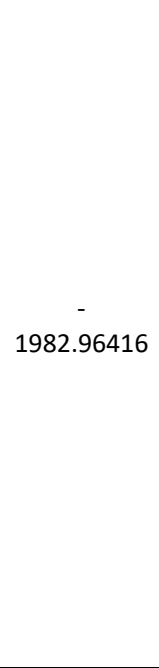 & 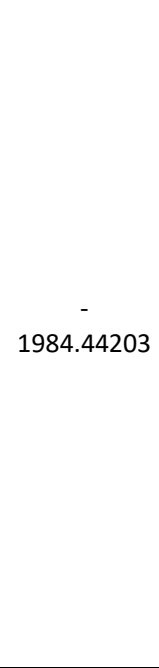 & 更 & $\begin{array}{c}- \\
1984.79221\end{array}$ & $\begin{array}{c}- \\
1984.44324\end{array}$ & 4.5 \\
\hline
\end{tabular}




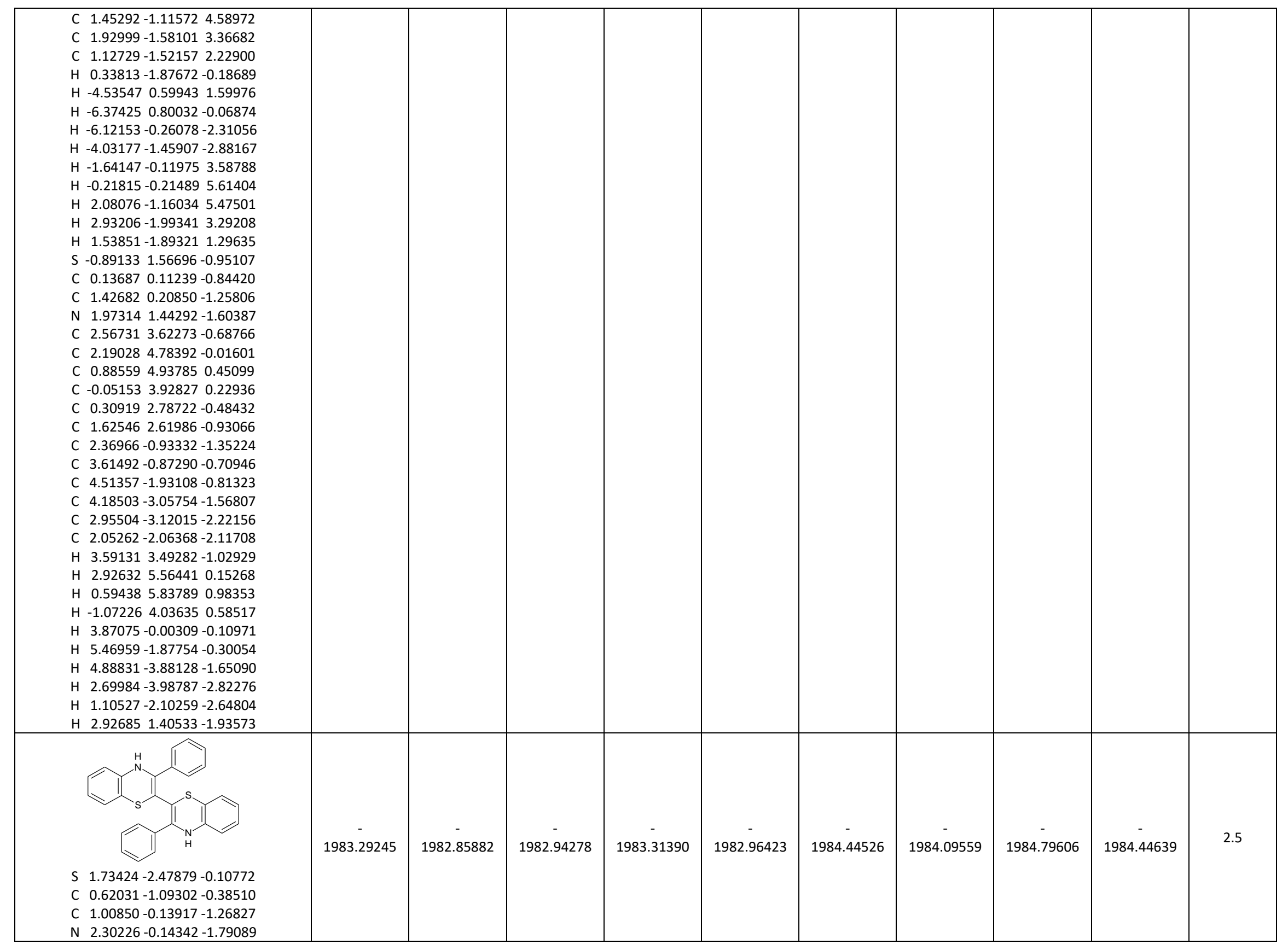




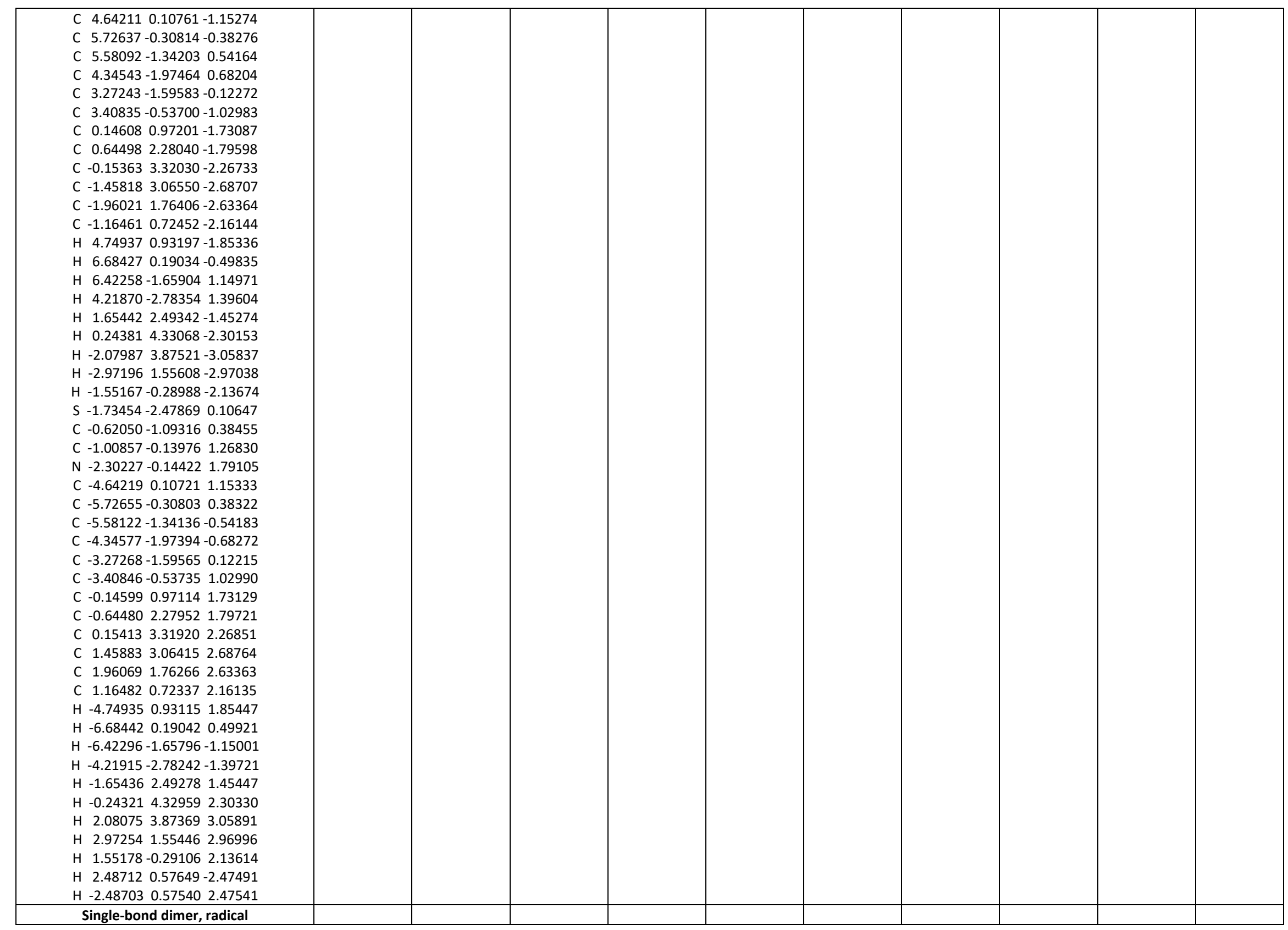




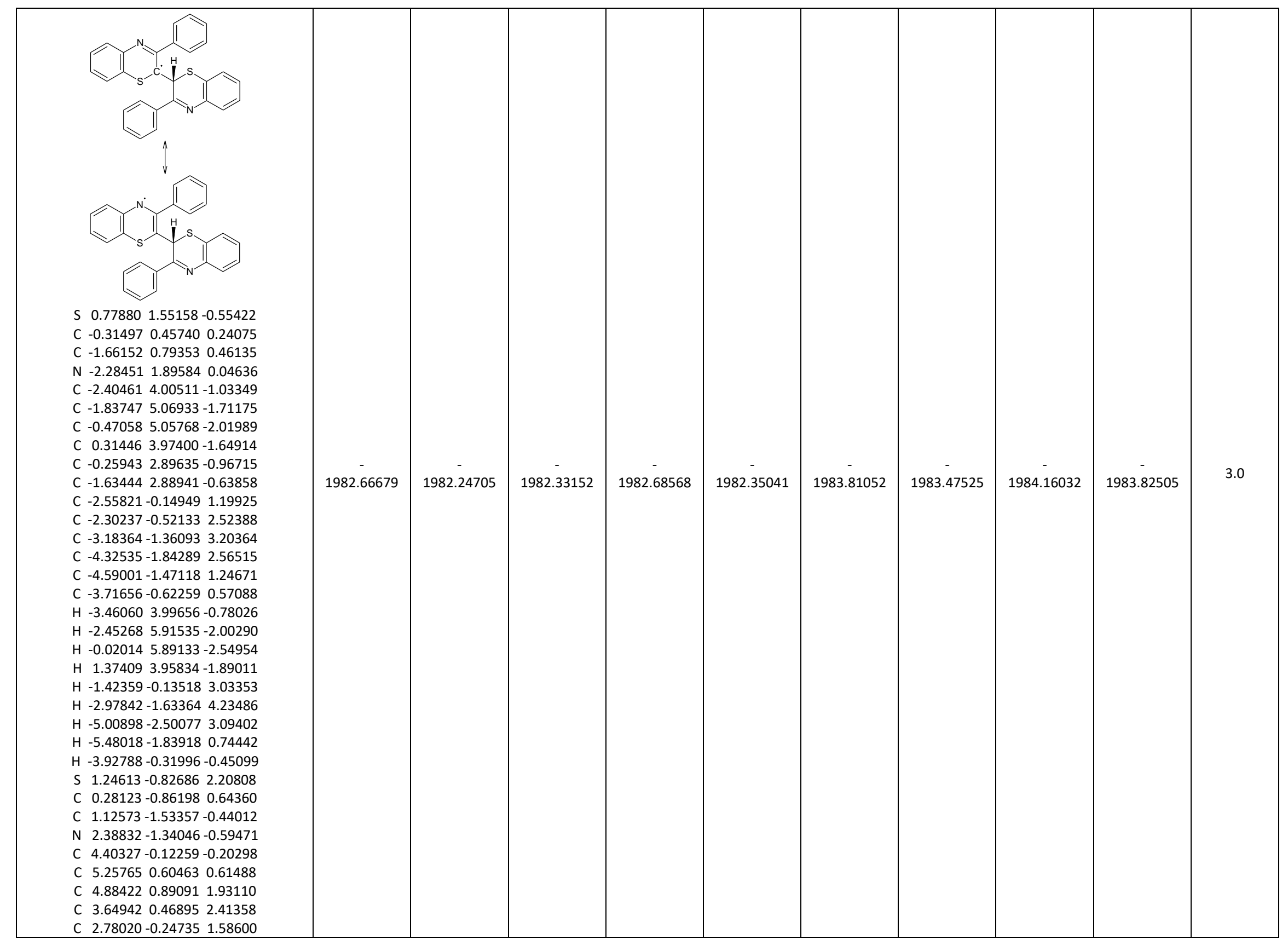




\begin{tabular}{|c|c|c|c|c|c|c|c|c|c|c|}
\hline $\begin{array}{lllll}\text { C } & 3.15595 & -0.56389 & 0.26482 \\
\text { C } & 0.43987 & -2.43785 & -1.39317 \\
\text { C } & -0.95808 & -2.52712 & -1.46417 \\
\text { C } & -1.57097 & -3.37626 & -2.38312 \\
\text { C } & -0.79948 & -4.15663 & -3.24086 \\
\text { C } & 0.59396 & -4.07945 & -3.17712 \\
\text { C } & 1.20628 & -3.22858 & -2.26643 \\
\text { H } & -0.54129 & -1.53092 & 0.90048 \\
\text { H } & 4.68026 & -0.37095 & -1.22350 \\
\text { H } & 6.21732 & 0.94041 & 0.23381 \\
\text { H } & 5.55130 & 1.44968 & 2.58074 \\
\text { H } & 3.35020 & 0.70252 & 3.43163 \\
\text { H } & -1.59023 & -1.92911 & -0.81550 \\
\text { H } & -2.65510 & -3.42544 & -2.42546 \\
\text { H } & -1.27828 & -4.82242 & -3.95310 \\
\text { H } & 1.20357 & -4.68788 & -3.83908 \\
\text { H } & 2.28784 & -3.16371 & -2.21215 \\
\end{array}$ & . & & & & & & & & & \\
\hline 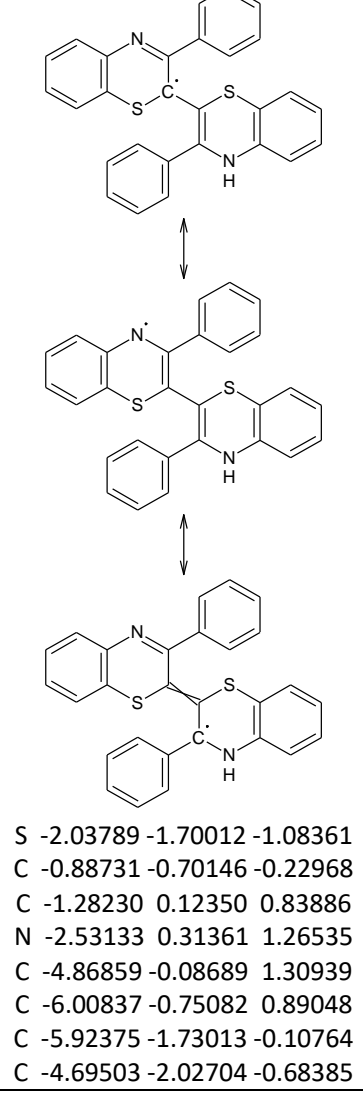 & $\begin{array}{l}- \\
1982.66840\end{array}$ & $\begin{array}{c}- \\
1982.24840\end{array}$ & 要 & 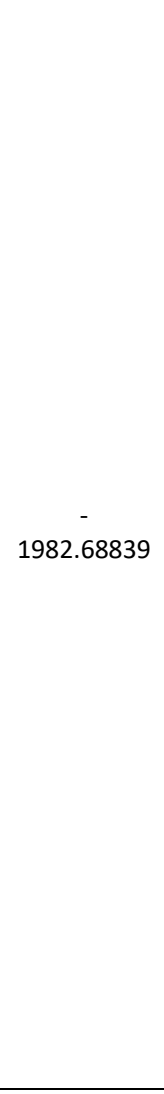 & 要 & 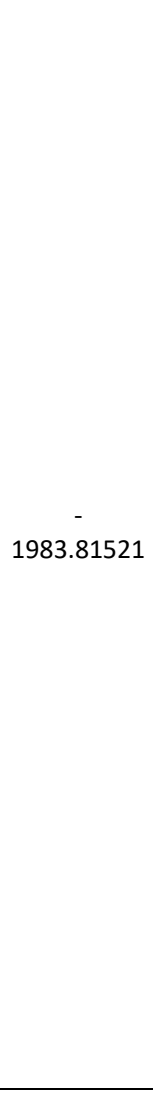 & 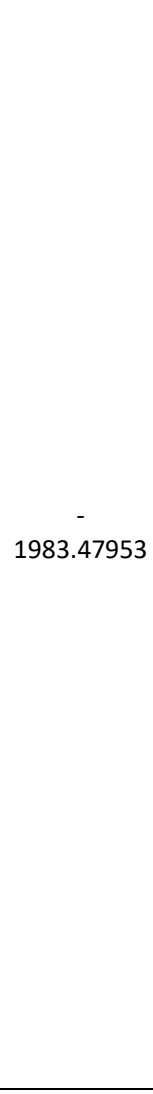 & 列 & 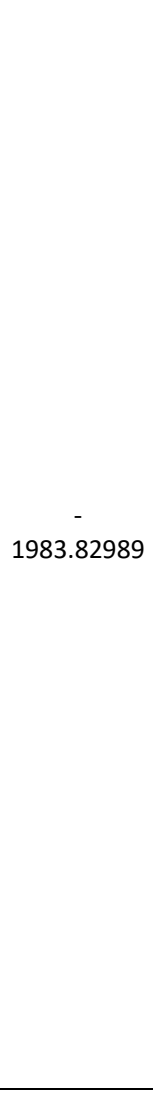 & 0.0 \\
\hline
\end{tabular}




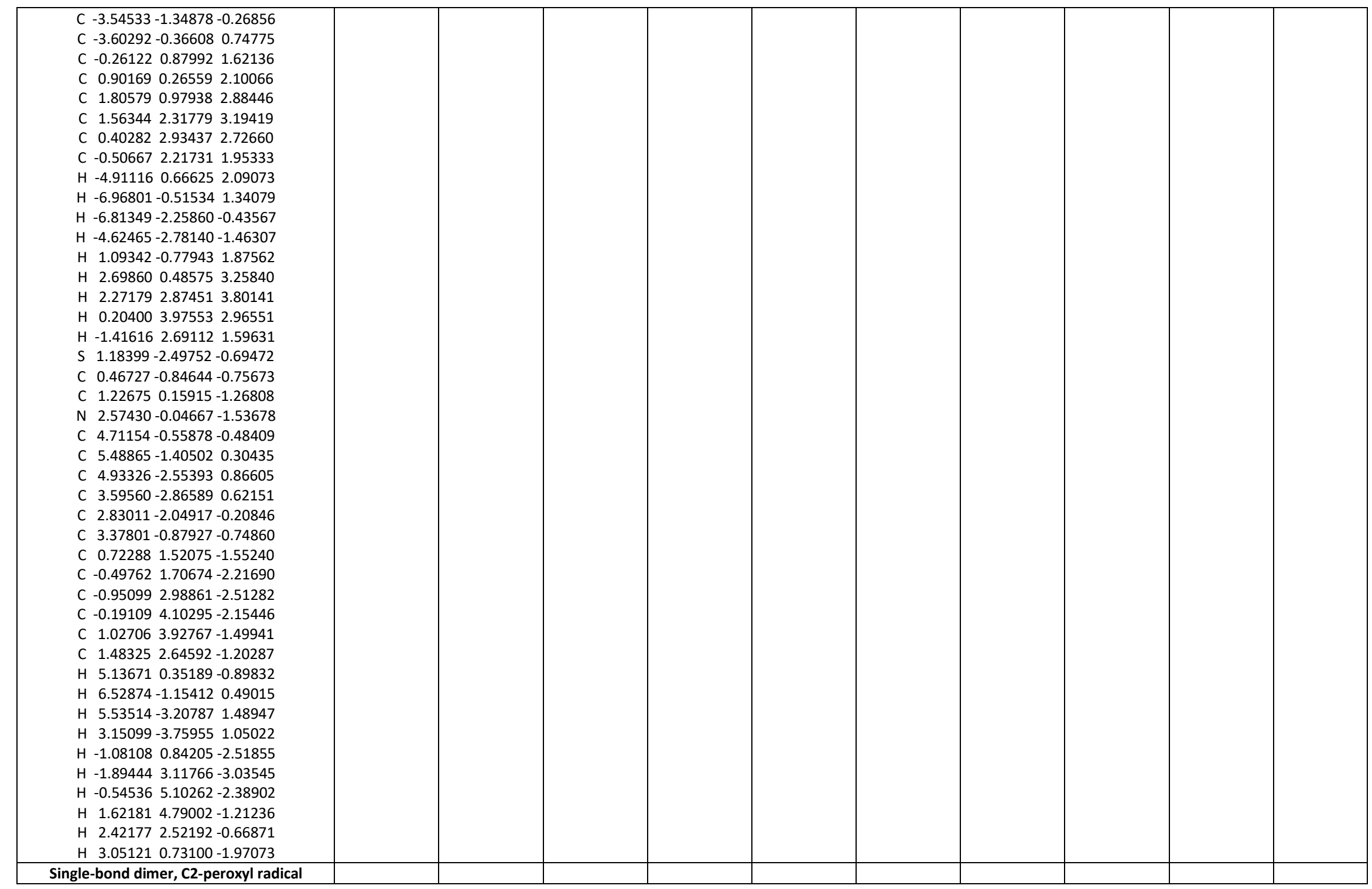




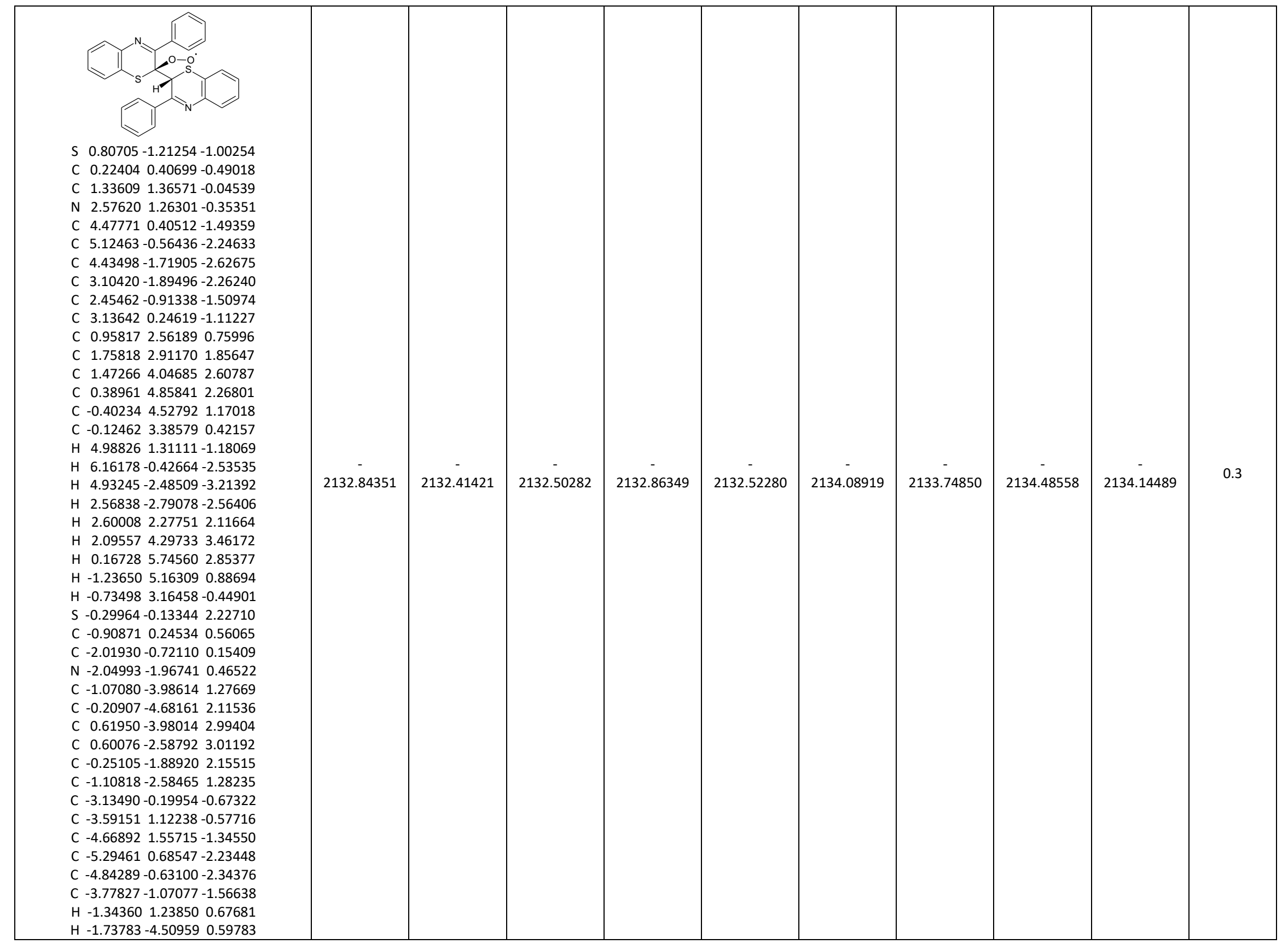




\begin{tabular}{|c|c|c|c|c|c|c|c|c|c|c|}
\hline $\begin{array}{lrrr}H & -0.19003 & -5.76691 & 2.09413 \\
H & 1.28751 & -4.51497 & 3.66251 \\
H & 1.25611 & -2.04118 & 3.68441 \\
H & -3.13719 & 1.82255 & 0.11748 \\
H & -5.01877 & 2.58041 & -1.24611 \\
H & -6.12809 & 1.02940 & -2.84004 \\
H & -5.31981 & -1.31499 & -3.03986 \\
H & -3.42199 & -2.09266 & -1.64602 \\
\text { O } & -0.48516 & 1.08164 & -1.62885 \\
O & 0.27147 & 1.21343 & -2.67804 \\
\end{array}$ & & & & & & & & & & \\
\hline 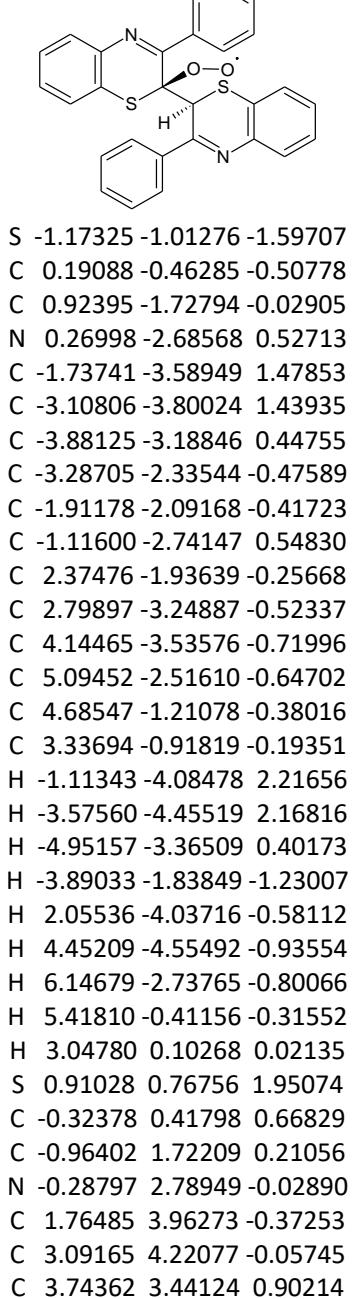 & $\begin{array}{c}- \\
2132.84353\end{array}$ & 2132.41399 & $\begin{array}{c}- \\
- \\
2132.50126\end{array}$ & $\begin{array}{c}- \\
2132.86278\end{array}$ & $\begin{array}{c}- \\
- \\
2132.52051\end{array}$ & 2134.08990 & $\begin{array}{c}- \\
2133.74763\end{array}$ & $\stackrel{-}{-}$ & 2134.14400 & 0.8 \\
\hline
\end{tabular}




\begin{tabular}{|c|c|c|c|c|c|c|c|c|c|c|}
\hline $\begin{array}{lrrrr}C & 3.07663 & 2.38403 & 1.51327 \\
\text { C } & 1.75147 & 2.10092 & 1.17090 \\
\text { C } & 1.06940 & 2.90757 & 0.23767 \\
\text { C } & -2.43188 & 1.78551 & 0.01751 \\
\text { C } & -2.95552 & 2.68735 & -0.92311 \\
\text { C } & -4.32754 & 2.80261 & -1.10650 \\
\text { C } & -5.20541 & 2.03133 & -0.34133 \\
\text { C } & -4.69717 & 1.14166 & 0.60226 \\
\text { C } & -3.32068 & 1.01121 & 0.77510 \\
\text { H } & -1.06288 & -0.19744 & 1.18653 \\
\text { H } & 1.22942 & 4.57649 & -1.09110 \\
\text { H } & 3.61469 & 5.03856 & -0.54369 \\
\text { H } & 4.77483 & 3.65063 & 1.17017 \\
\text { H } & 3.58735 & 1.76579 & 2.24671 \\
\text { H } & -2.26781 & 3.28501 & -1.51242 \\
\text { H } & -4.71540 & 3.49411 & -1.84900 \\
\text { H } & -6.27855 & 2.12419 & -0.48121 \\
\text { H } & -5.37143 & 0.54398 & 1.20865 \\
\text { H } & -2.95672 & 0.31973 & 1.52882 \\
\text { O } & 1.09083 & 0.33067 & -1.31612 \\
\text { O } & 0.59786 & 0.68155 & -2.47311 \\
\end{array}$ & & & & & & & & & & \\
\hline \begin{tabular}{llll} 
& \multicolumn{3}{c}{$\mathrm{H}$} \\
S & -2.13102 & -0.78424 & -1.29878 \\
C & -0.91808 & -0.29379 & -0.04940 \\
C & -1.21079 & 1.09211 & 0.55749 \\
N & -2.36882 & 1.64186 & 0.61174 \\
C & -4.72987 & 1.80263 & 0.38776 \\
C & -5.94652 & 1.38786 & -0.13567 \\
C & -5.98916 & 0.29868 & -1.01024 \\
C & -4.81996 & -0.37682 & -1.34617 \\
C & -3.59905 & 0.03761 & -0.80935 \\
C & -3.53735 & 1.13915 & 0.05875 \\
C & -0.09943 & 1.81013 & 1.23546 \\
C & 0.91377 & 1.14370 & 1.93807 \\
C & 1.89941 & 1.86651 & 2.60816 \\
C & 1.89658 & 3.25928 & 2.57577 \\
C & 0.89192 & 3.93091 & 1.87640 \\
C & -0.09987 & 3.21272 & 1.21869 \\
H & -4.66683 & 2.65008 & 1.06408 \\
H & -6.85956 & 1.91123 & 0.13060 \\
H & -6.93546 & -0.03081 & -1.42879 \\
H & -4.85470 & -1.22908 & -2.01934 \\
H & 0.92614 & 0.06076 & 1.99072 \\
\end{tabular} & $\begin{array}{c}- \\
2132.84351\end{array}$ & $\begin{array}{c}- \\
- \\
- \\
2132.41373\end{array}$ & $\begin{array}{c}-2 \\
- \\
- \\
2132.50175\end{array}$ & - & $\begin{array}{l}- \\
- \\
2132.52184\end{array}$ & 2134.09018 & $\begin{array}{l}- \\
- \\
2133.74843\end{array}$ & $\begin{array}{c}- \\
2134.48706\end{array}$ & $\begin{array}{c}- \\
- \\
- \\
2134.14531\end{array}$ & 0.0 \\
\hline
\end{tabular}




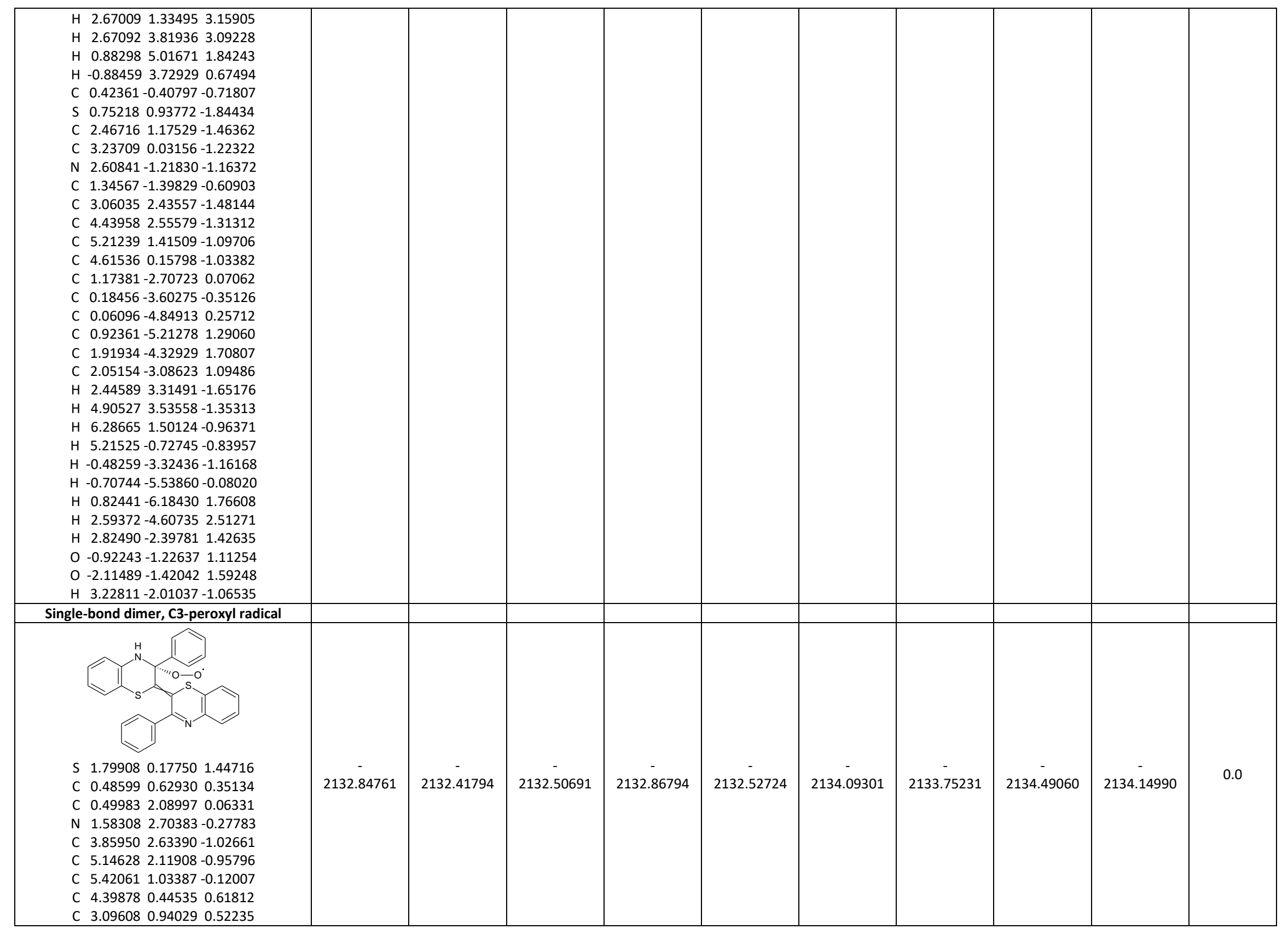




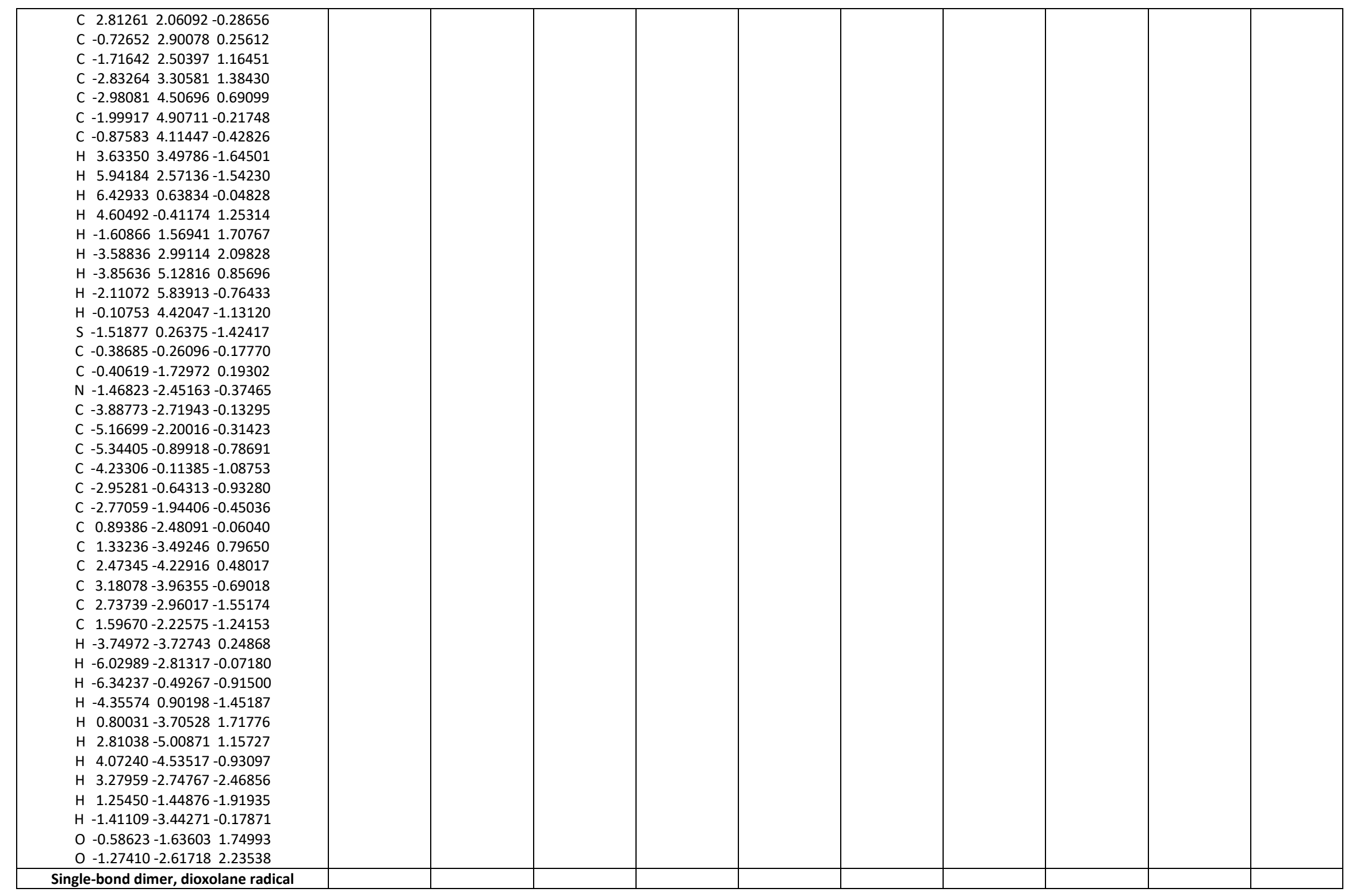




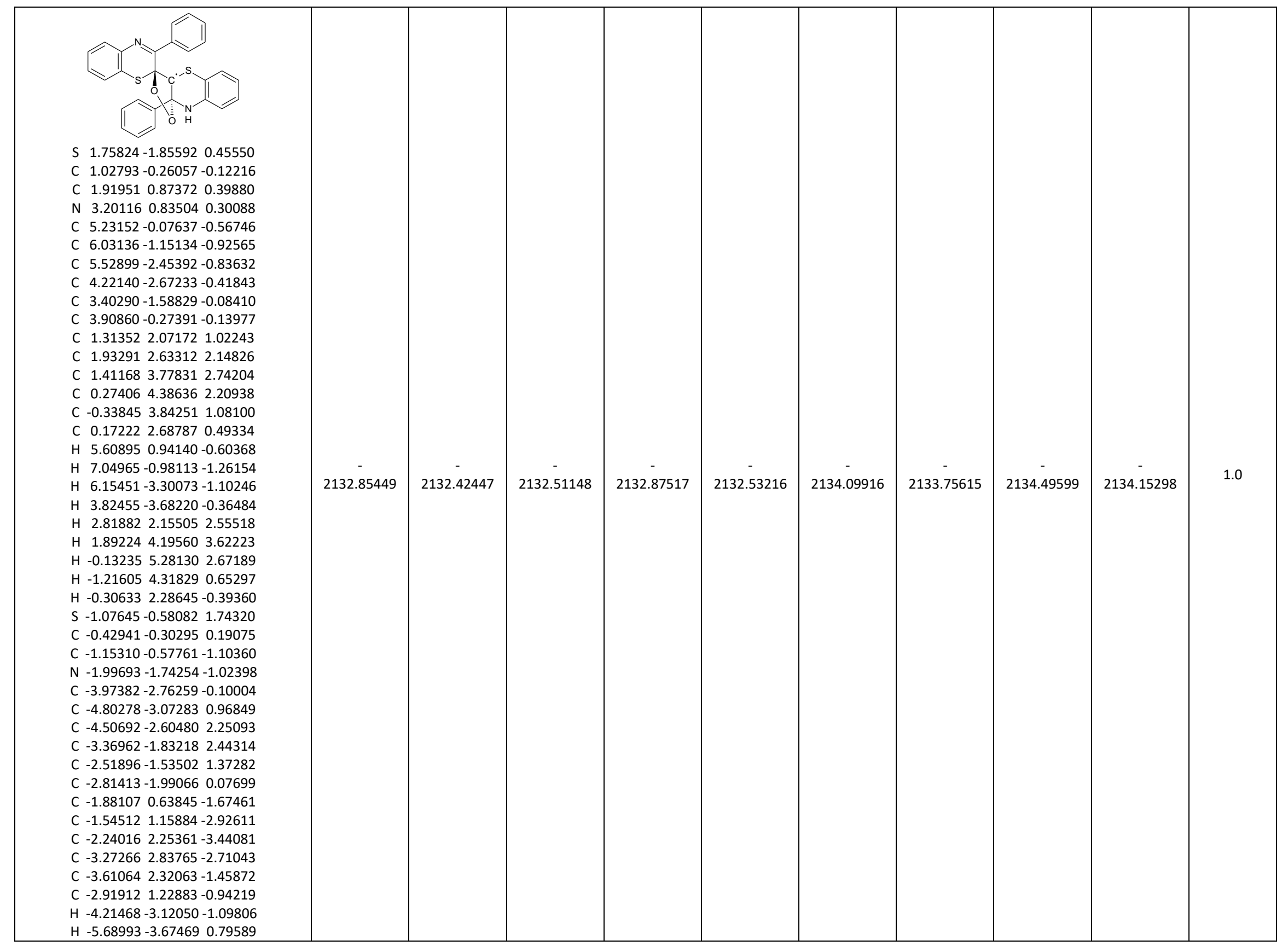




\begin{tabular}{|c|c|c|c|c|c|c|c|c|c|c|}
\hline $\begin{array}{llll}\text { H } & -5.15520 & -2.83501 & 3.09008 \\
\text { H } & -3.12703 & -1.45493 & 3.43327 \\
\text { H } & -0.73910 & 0.70834 & -3.49526 \\
\text { H } & -1.96924 & 2.65016 & -4.41523 \\
\text { H } & -3.81094 & 3.69180 & -3.11104 \\
\text { H } & -4.41294 & 2.77035 & -0.88075 \\
\text { H } & -3.18973 & 0.84295 & 0.03701 \\
\text { O } & 1.03127 & -0.14185 & -1.53914 \\
\text { O } & -0.06384 & -0.96602 & -1.96514 \\
\text { H } & -2.46628 & -1.92658 & -1.90326 \\
\end{array}$ & & & & & & & & & & \\
\hline $\begin{array}{llll} & \end{array}$ & $\begin{array}{c}- \\
2132.85448\end{array}$ & 2132.42450 & $\begin{array}{c}- \\
2132.51041\end{array}$ & $\begin{array}{c}- \\
2132.87432\end{array}$ & $\begin{array}{c}- \\
2132.53026\end{array}$ & $\begin{array}{c}- \\
- \\
2134.09887\end{array}$ & $\begin{array}{c}- \\
2133.75481\end{array}$ & $\begin{array}{c}- \\
2134.49527\end{array}$ & - & 2.1 \\
\hline
\end{tabular}




\begin{tabular}{|c|c|c|c|c|c|c|c|c|c|c|}
\hline $\begin{array}{llll}C & -2.12997 & 1.48784 & -2.99451 \\
\text { C } & -1.68282 & 1.14632 & -1.71383 \\
\text { C } & -2.19063 & 1.80930 & -0.58041 \\
\text { C } & -2.59210 & -0.87858 & 1.04296 \\
\text { C } & -2.32574 & -2.25044 & 1.11054 \\
\text { C } & -3.36978 & -3.16715 & 1.17449 \\
\text { C } & -4.69288 & -2.72207 & 1.16908 \\
\text { C } & -4.96282 & -1.35753 & 1.10148 \\
\text { C } & -3.91600 & -0.43716 & 1.03800 \\
\text { H } & -3.50572 & 3.37175 & 0.08966 \\
\text { H } & -4.24878 & 4.00662 & -2.17561 \\
\text { H } & -3.38612 & 2.77340 & -4.16967 \\
\text { H } & -1.73618 & 0.95336 & -3.85515 \\
\text { H } & -1.29490 & -2.59549 & 1.11589 \\
\text { H } & -3.15152 & -4.22989 & 1.22659 \\
\text { H } & -5.50892 & -3.43750 & 1.21510 \\
\text { H } & -5.98938 & -1.00309 & 1.09524 \\
\text { H } & -4.14585 & 0.62155 & 0.97770 \\
\text { O } & 0.56878 & 0.39217 & 2.09394 \\
\text { O } & -0.82087 & 0.02778 & 2.28767 \\
\text { H } & -2.29739 & 1.92695 & 1.45050 \\
\end{array}$ & & & & & & & & & & \\
\hline 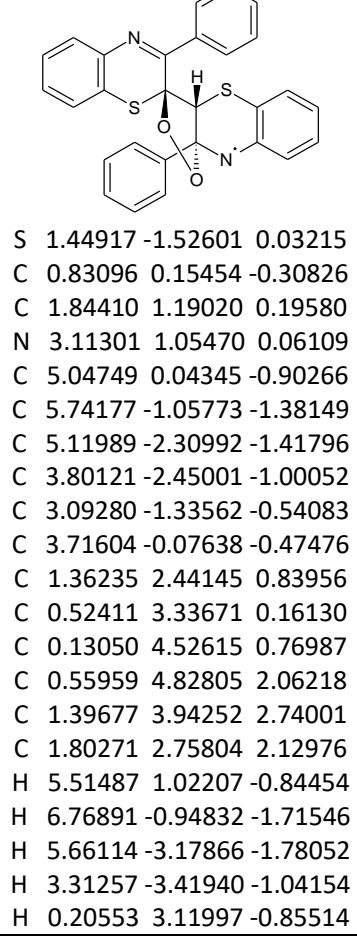 & $\begin{array}{l}- \\
- \\
- \\
2132.85417\end{array}$ & $\begin{array}{c}- \\
- \\
2132.42510\end{array}$ & $\begin{array}{c}- \\
- \\
2132.51257\end{array}$ & $\begin{array}{c}- \\
- \\
2132.87505\end{array}$ & $\begin{array}{c}- \\
- \\
2132.53345\end{array}$ & $\begin{array}{c}- \\
- \\
2134.09824\end{array}$ & $\begin{array}{c}- \\
2133.75664\end{array}$ & $\begin{array}{c}- \\
- \\
2134.49366\end{array}$ & $\begin{array}{c}- \\
2134.15207\end{array}$ & 1.6 \\
\hline
\end{tabular}




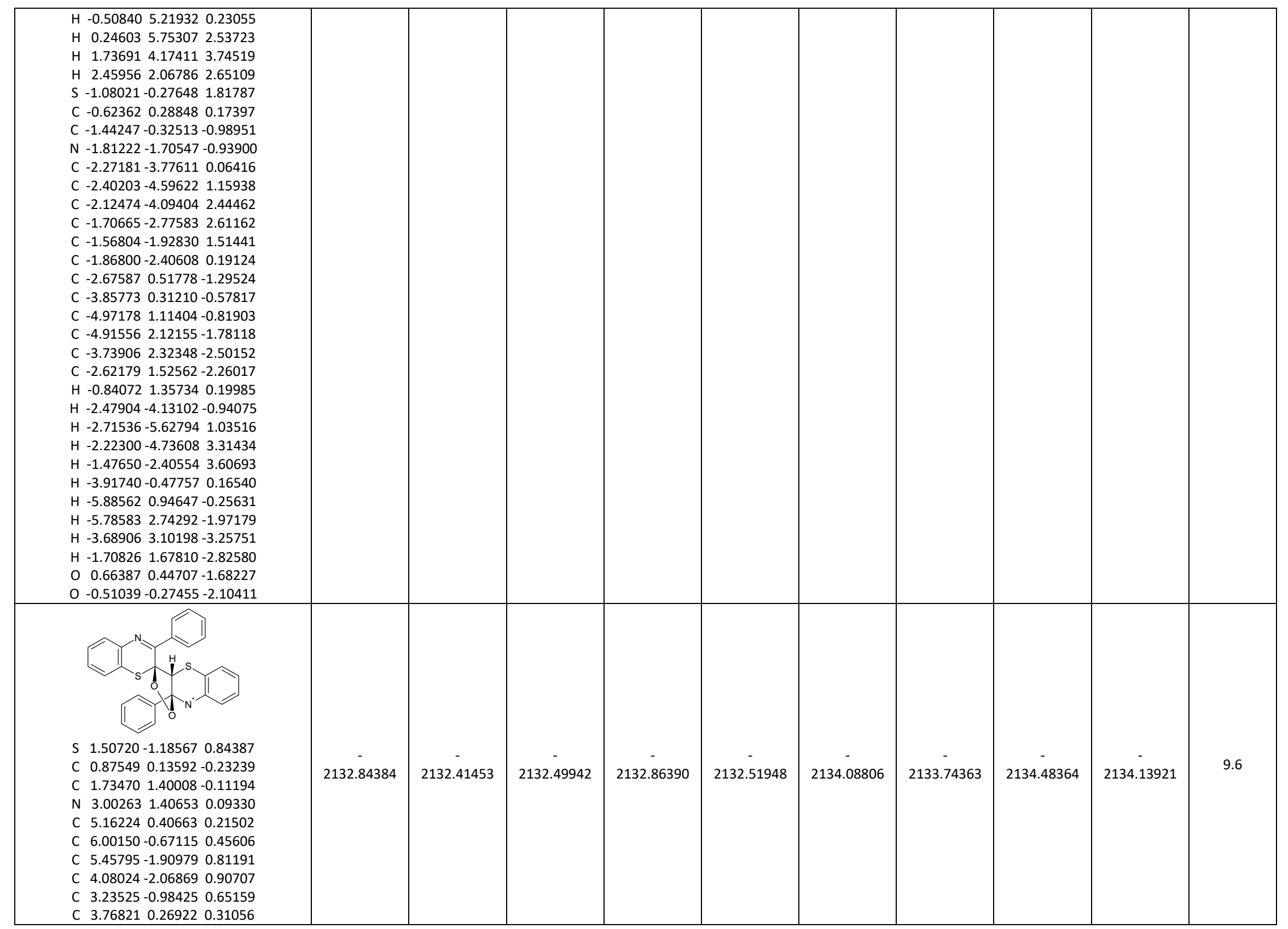


C $1.098522 .72950-0.32297$

C $0.320793 .00885-1.45579$

C $-0.207374 .28395-1.64574$

C $0.023445 .28800-0.70595$

C 0.797285 .016060 .42209

C 1.337743 .746680 .60909

H $5.559071 .38180-0.05171$

H $7.07730-0.550390 .37477$

H $6.10887-2.756341 .00876$

H $3.65534-3.032951 .17193$

H $0.14932 \quad 2.23948-2.20356$

H $-0.797064 .49262-2.53370$

H $-0.395356 .27919-0.85404$

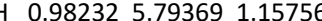

H 1.947613 .531951 .48162

S - -1.306860 .153731 .66223$

C $-0.61642 \quad 0.295010 .01159$

C $-1.25102-0.67095-0.99679$

$\mathrm{N}-2.59580-0.29529-1.29133$

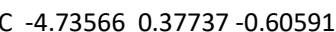

$\begin{array}{llll}C & -5.66187 & 0.73634 & 0.34531\end{array}$

C -5.263400 .882191 .68516$

C -3.93158 0.692632 .04788

C -2.971990 .335621 .10325$

C $-3.364850 .12845-0.27541$

$-1.18670-2.18286-0.75962$

C $-0.37853-2.97682-1.58018$

C $-0.33136-4.35788-1.40639$

C $-1.09413-4.96811-0.41258$

C $-1.91891-4.186550 .39425$

C $-1.97358-2.805730 .21595$

H $-0.89608 \quad 1.28870-0.34889$

$-5.014830 .24398-1.64660$

H -6.696320 .90428$

$\begin{array}{llll}H & -5.98715 & 1.16337 & 2.44366\end{array}$

H -3.62983 0.842793 .08085

H $0.22247-2.51118-2.35234$

$0.30552-4.95645-2.05154$

$-1.05620-6.04505-0.27558$

H $-2.53321-4.650381 .16061$

H $-2.64625-2.227990 .83944$

O $0.90246-0.23691-1.63696$

O $-0.45806-0.31273-2.14135$

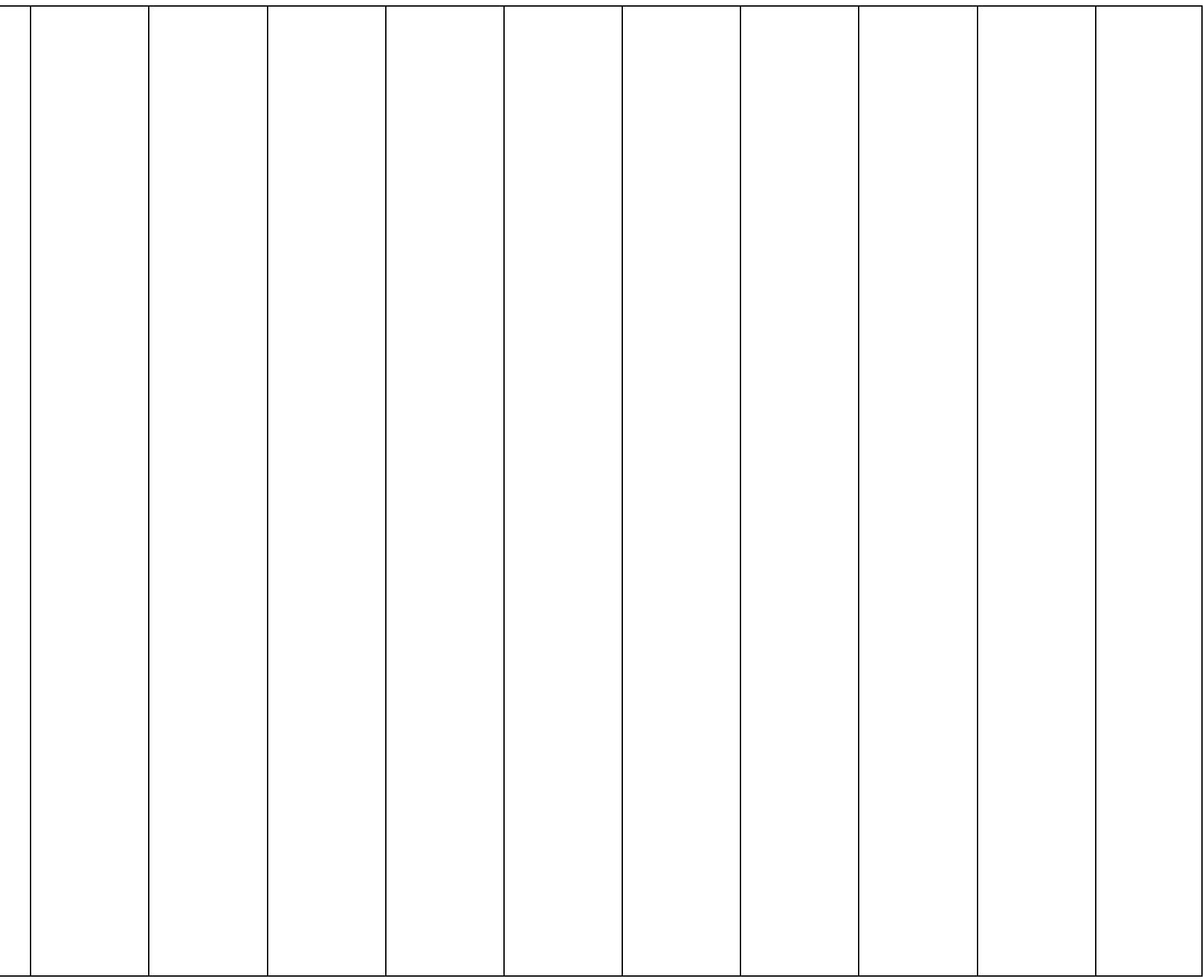




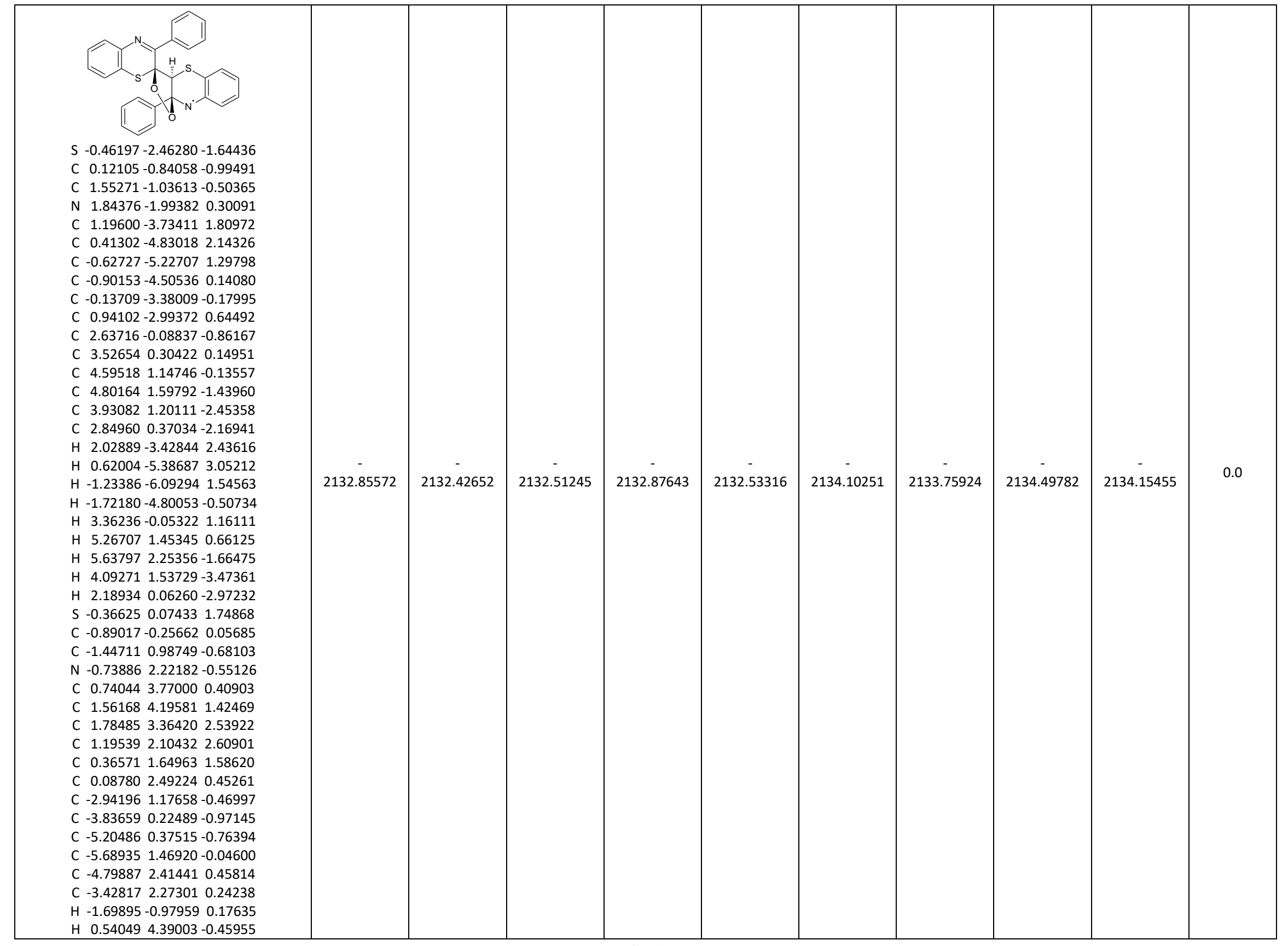




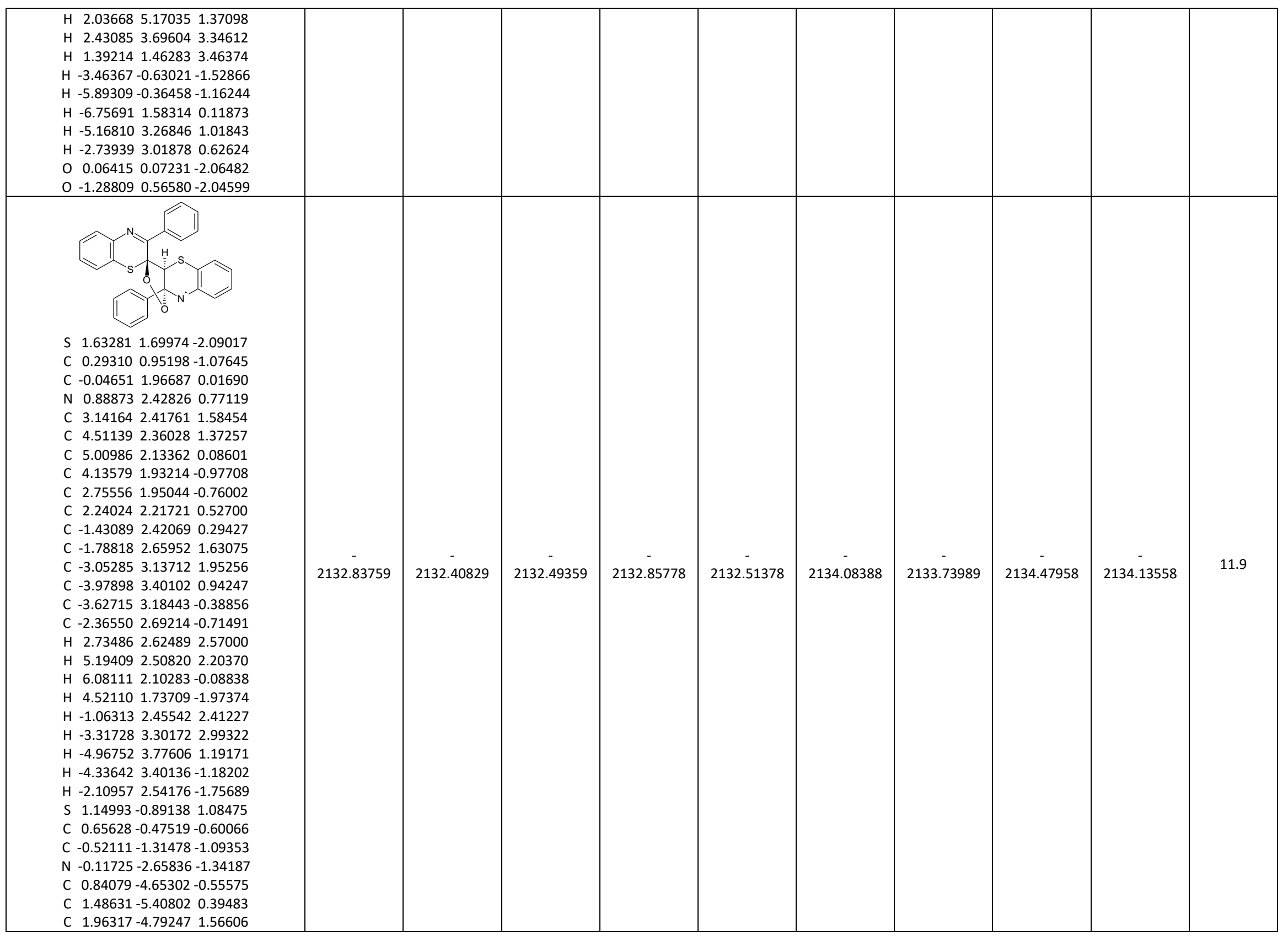




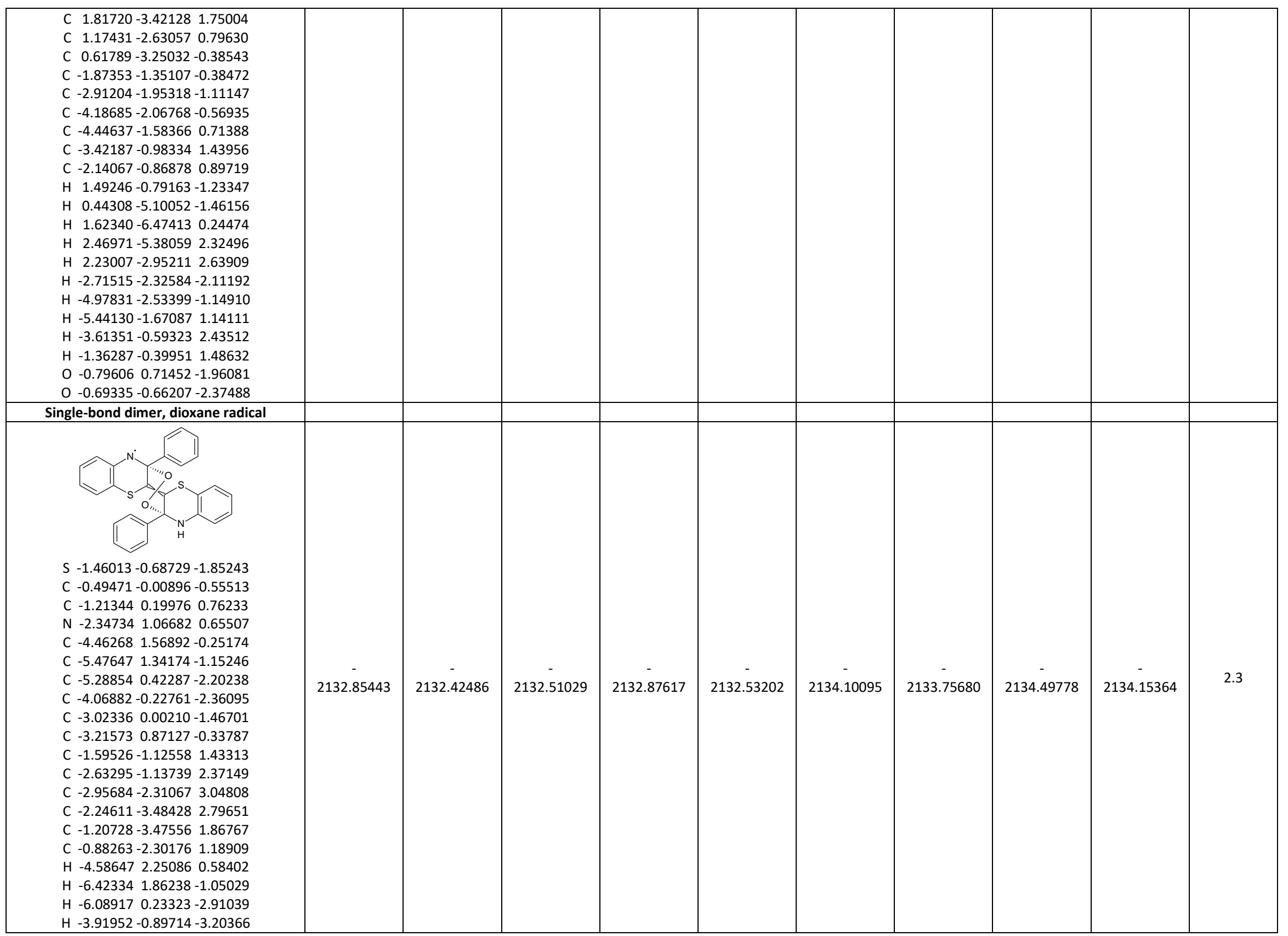




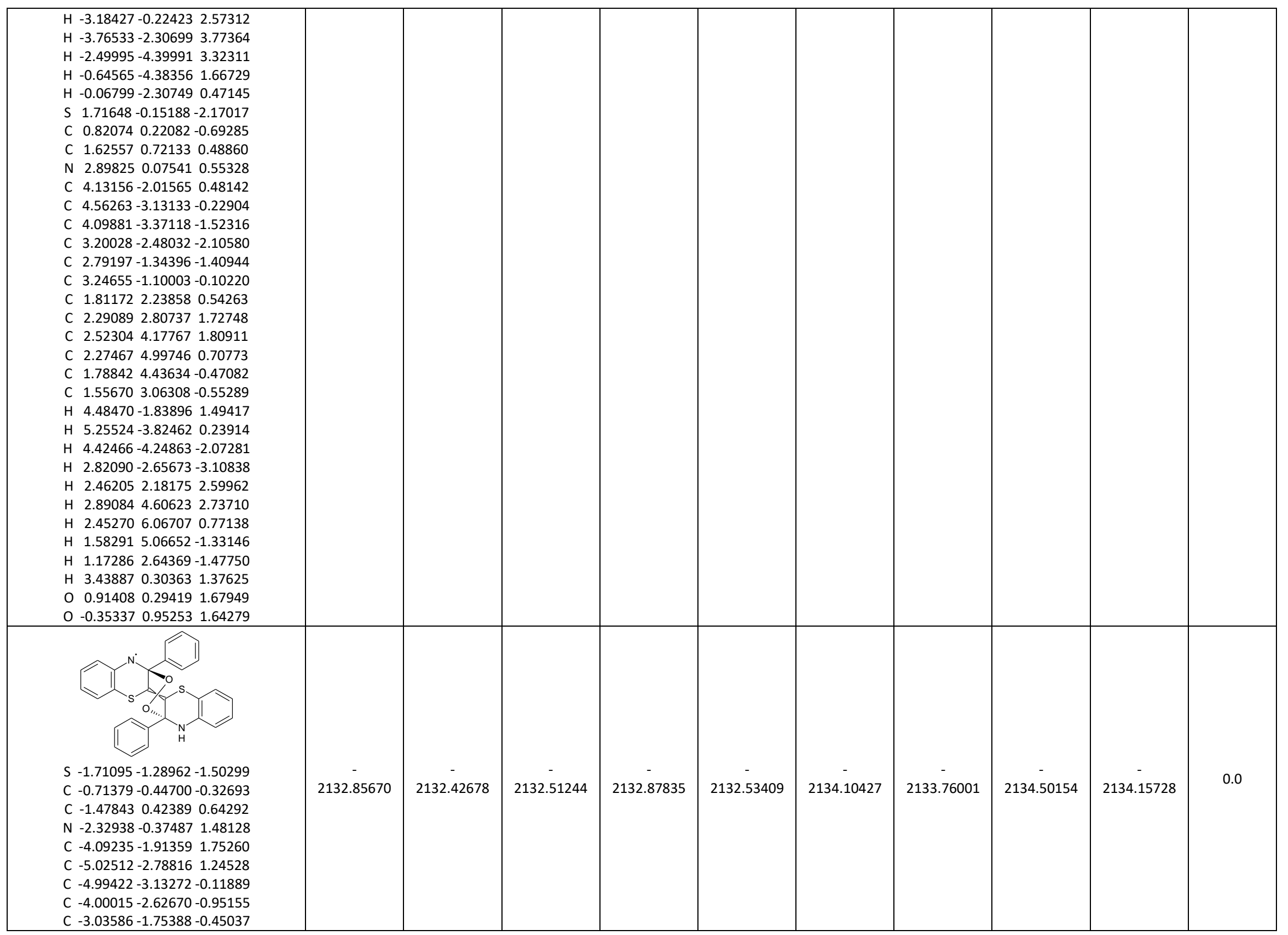




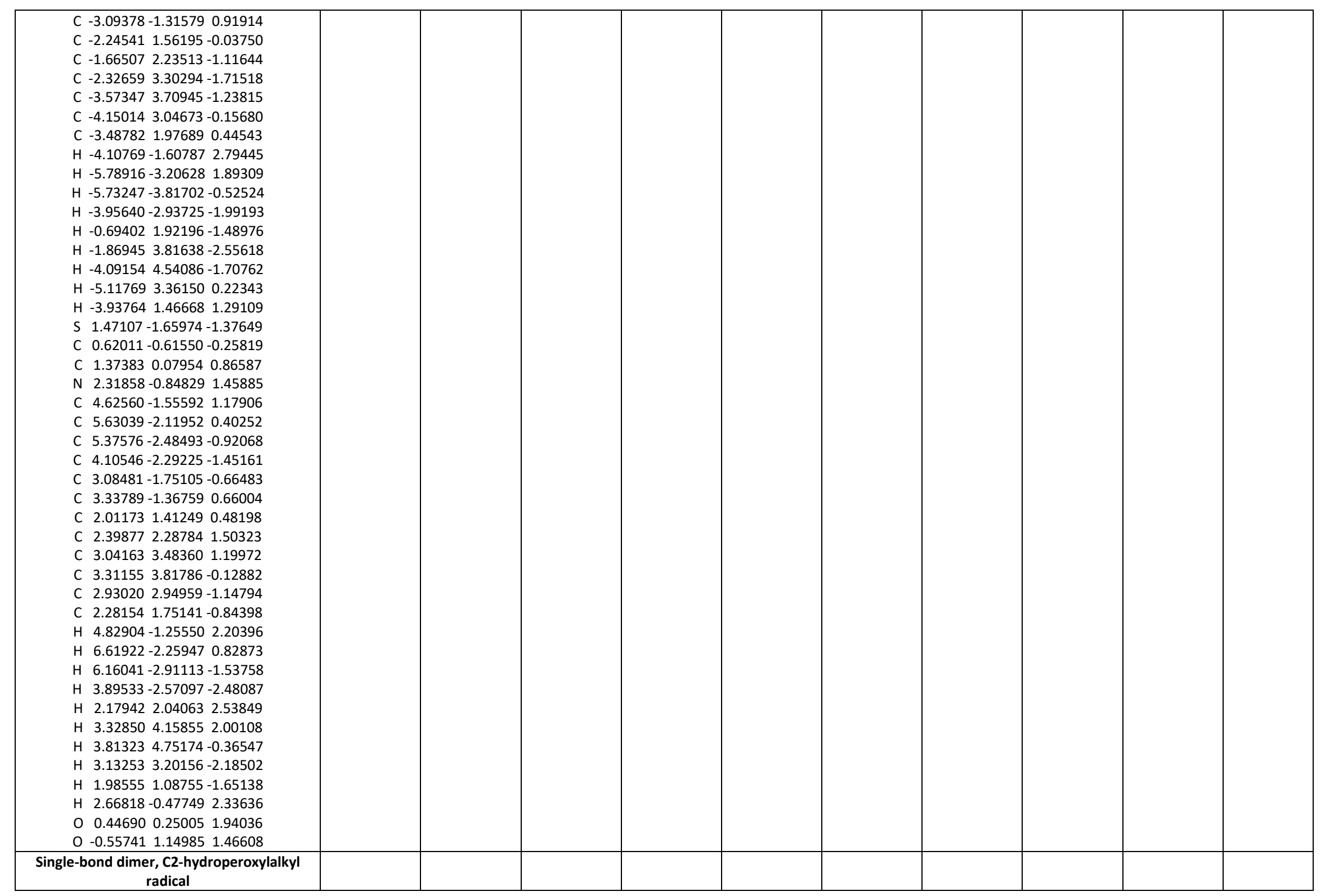




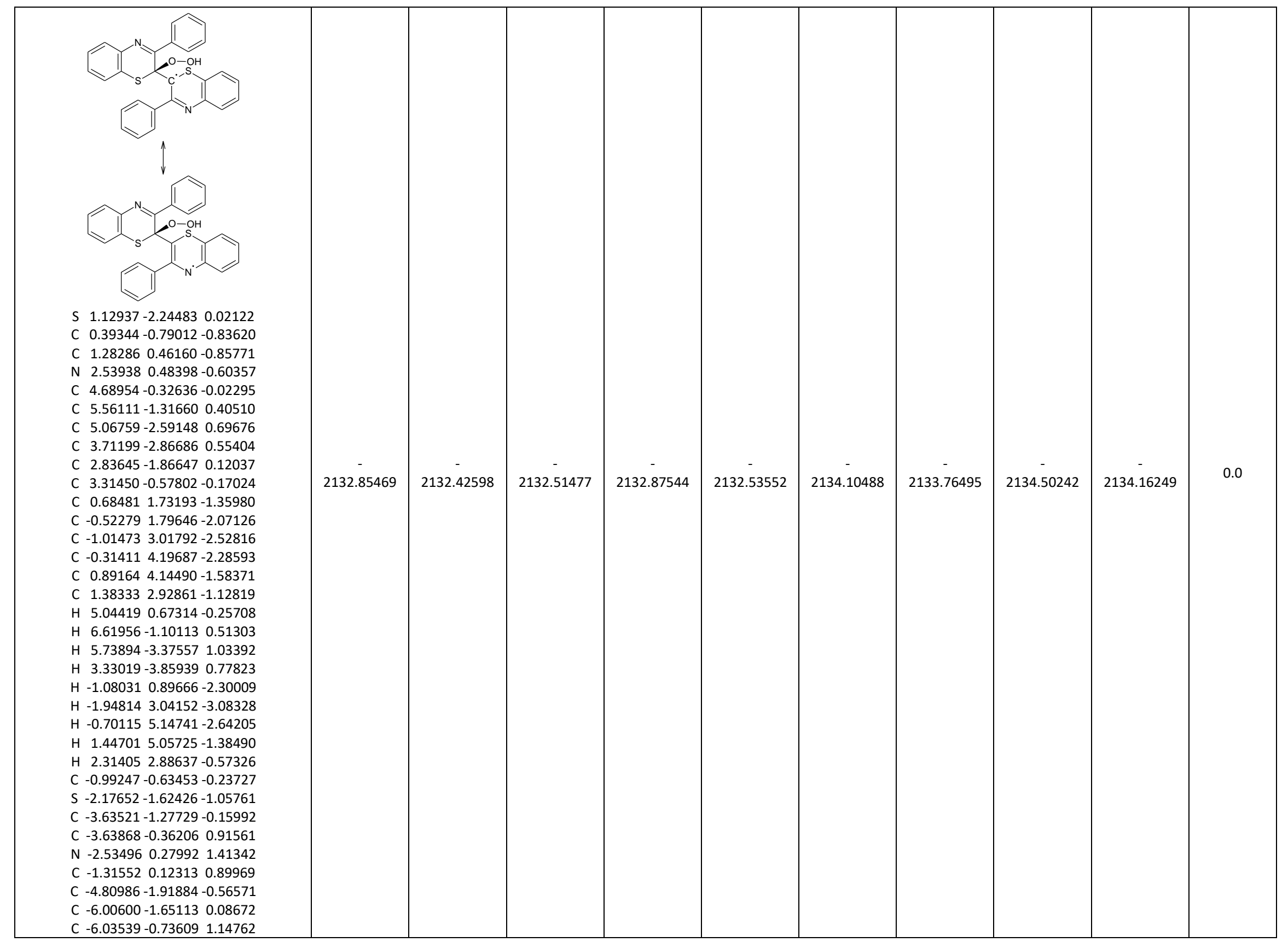




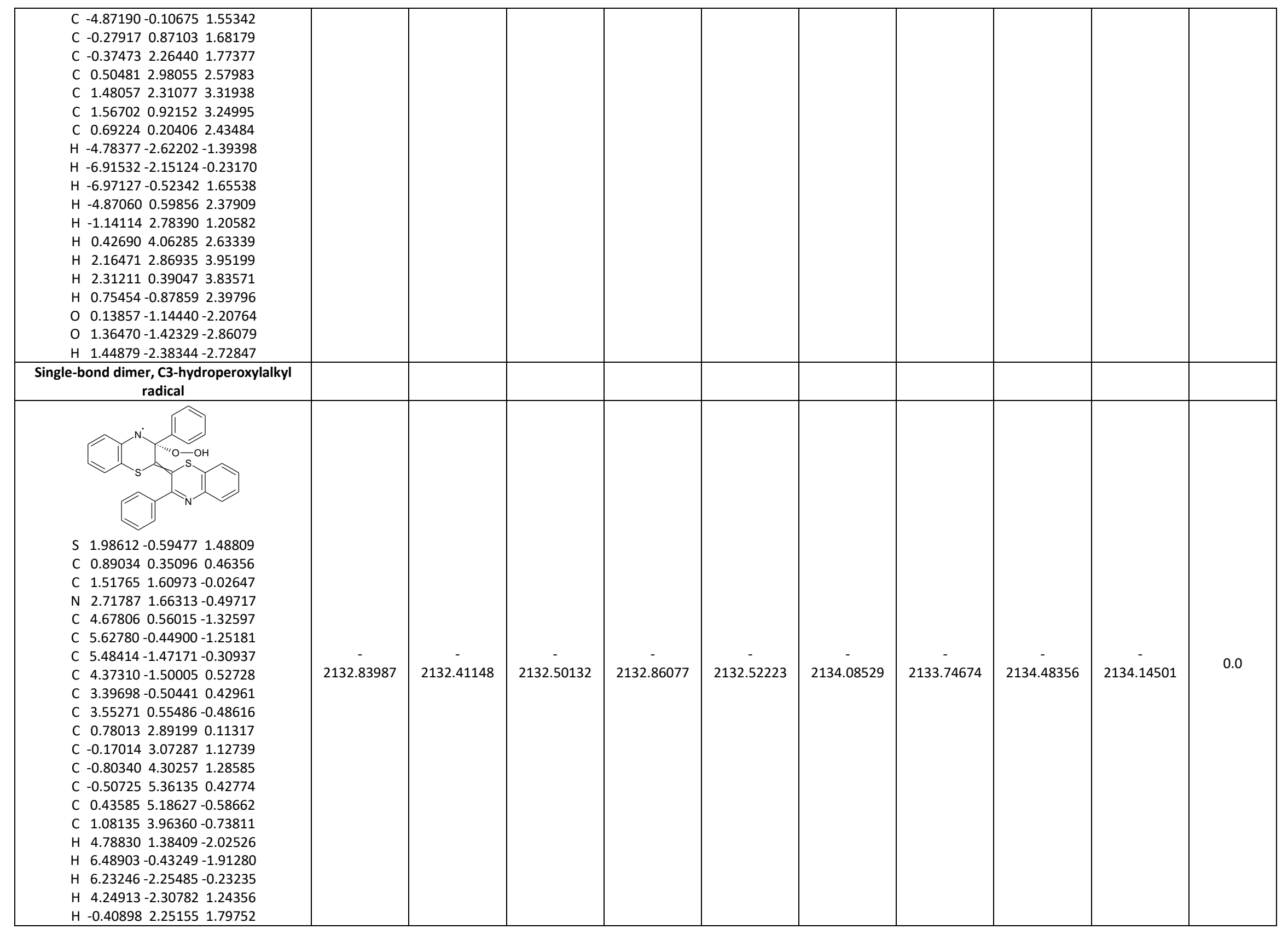




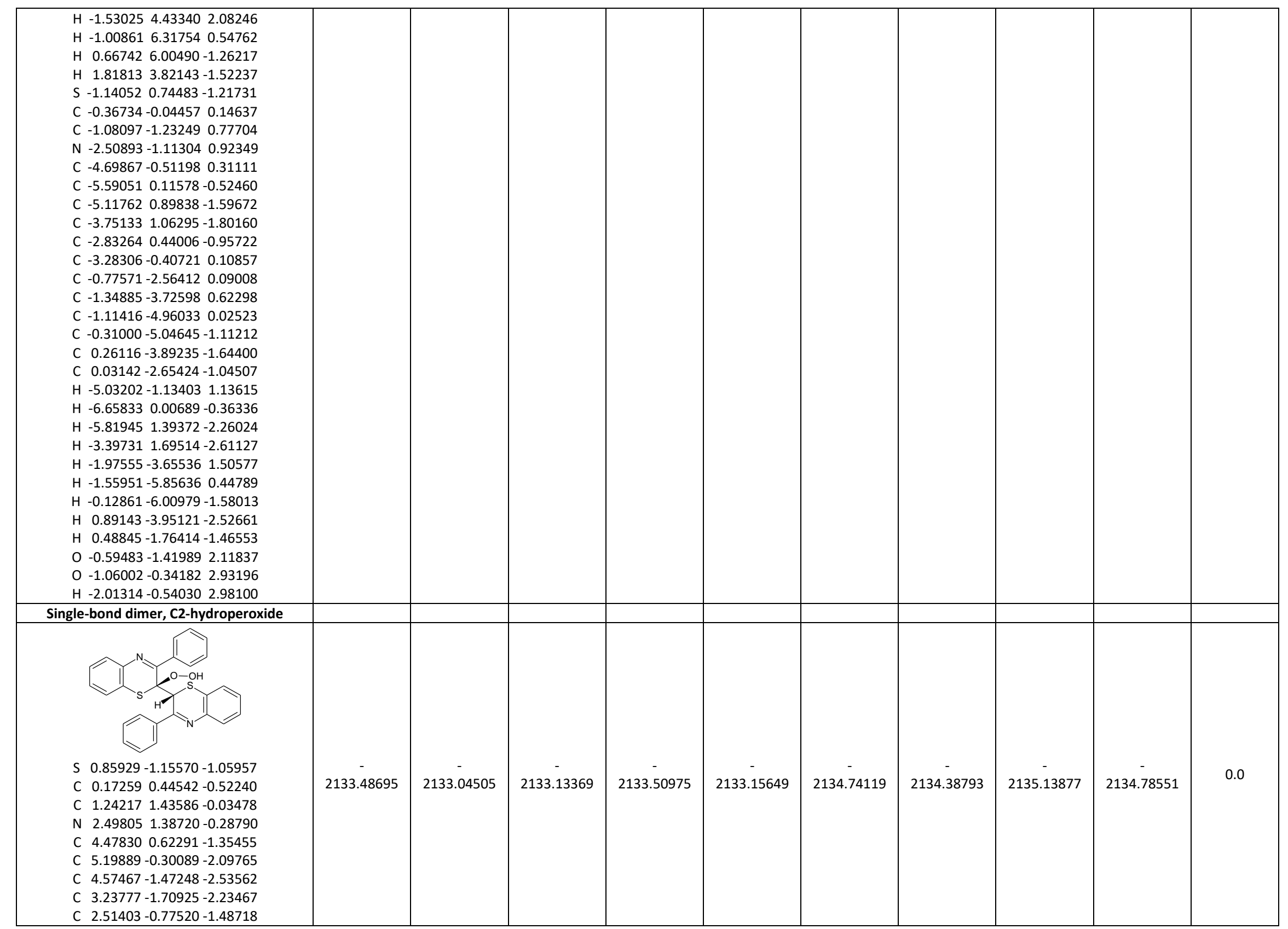


C $3.12858 \quad 0.40297-1.03658$

C $0.789532 .61506 \quad 0.75897$

C $-0.32135 \quad 3.391850 .39889$

C $-0.665964 .51698 \quad 1.14622$

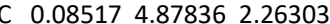

C 1.194554 .113332 .62528

C 1.546252 .995191 .87654

H $4.936321 .54167-0.99996$

H $6.24153-0.11429-2.33531$

H $5.12861-2.20398-3.11641$

2.75262-2.61884-2.57868

H $-0.898753 .13782-0.48446$

H $-1.52125 \quad 5.11592 \quad 0.84675$

$\begin{array}{llll}H & -0.18937 & 5.75282 & 2.84595\end{array}$

H $1.78597 \quad 4.386843 .49446$

H 2.408922 .397672 .15479

$\begin{array}{llll}\text { C } & -0.91531 & 0.17913 & 0.56632\end{array}$

S $-0.23286-0.21150 \quad 2.20071$

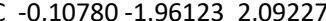

C $-0.95279-2.677101 .22435$

N - $-1.94224-2.087910 .44406$

C $-1.98260-0.834180 .16379$

C $0.79394-2.637822 .91500$

C $0.87642-4.027082 .86608$

C $0.06137-4.747511 .99012$

$\begin{array}{ll}0.05069-4.07485 & 1.18663\end{array}$

C $-3.14746-0.35484-0.62088$

$-3.78159-1.24554-1.50160$

C $-4.88970-0.84698-2.23929$

C $-5.395730 .44702-2.10176$

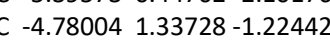

C -3.65950 $0.94406-0.49613$

H $1.43843-2.075663 .58535$

H $1.58324-4.544163 .50816$

H $0.12981-5.830091 .94425$

H - $-1.50899-4.614370 .51177$

H -3.38381 -2.25006-1.60276

H $-5.35884-1.54563-2.92615$

H $-6.263420 .75869-2.67602$

H $-5.171152 .34314-1.10302$

H -3.212491 .658240 .18850$

O $-0.584561 .04637-1.57425$

O $0.296621 .41932-2.62905$

H $0.229460 .65275-3.22287$

H $-1.39893 \quad 1.14358 \quad 0.72497$

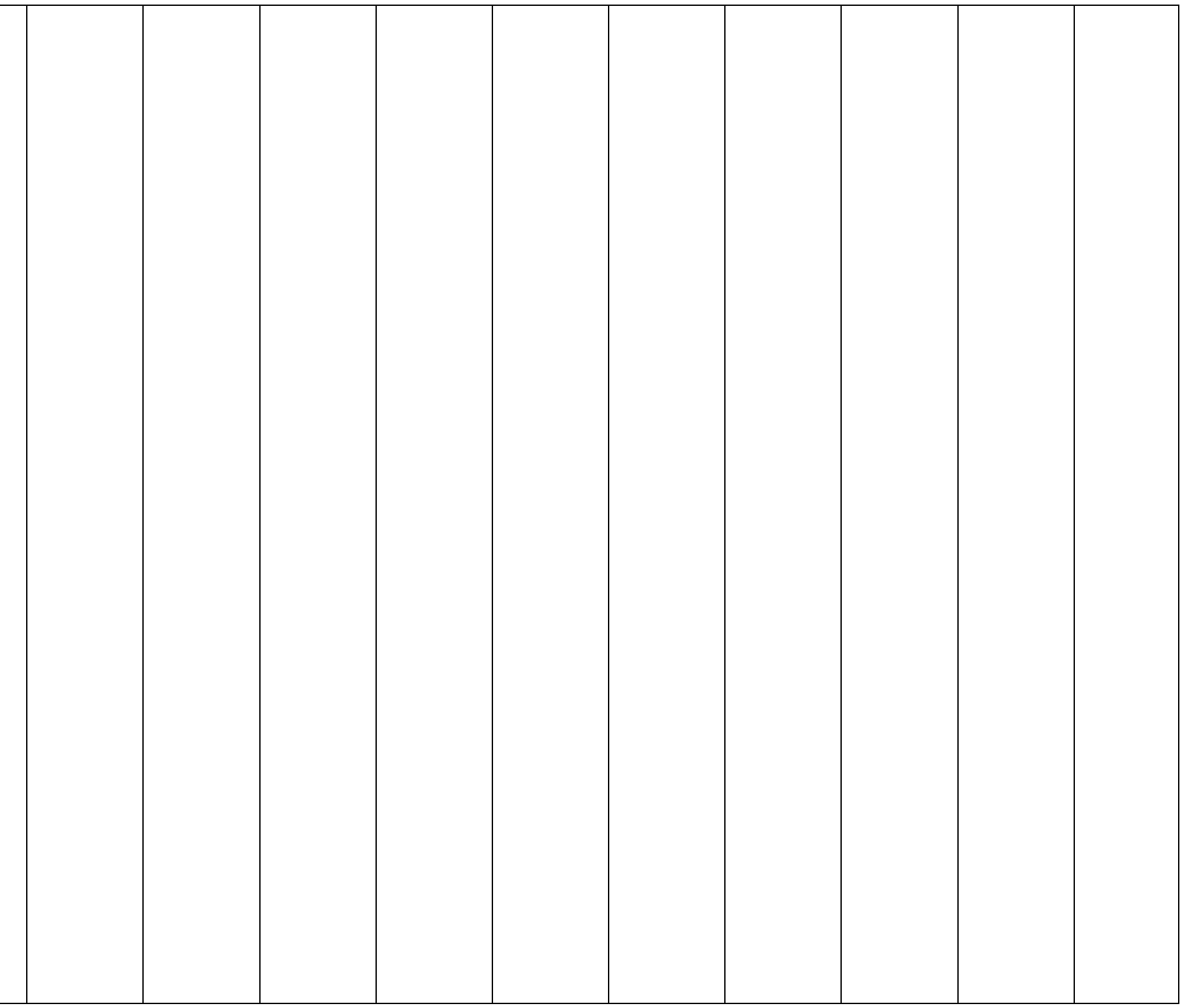




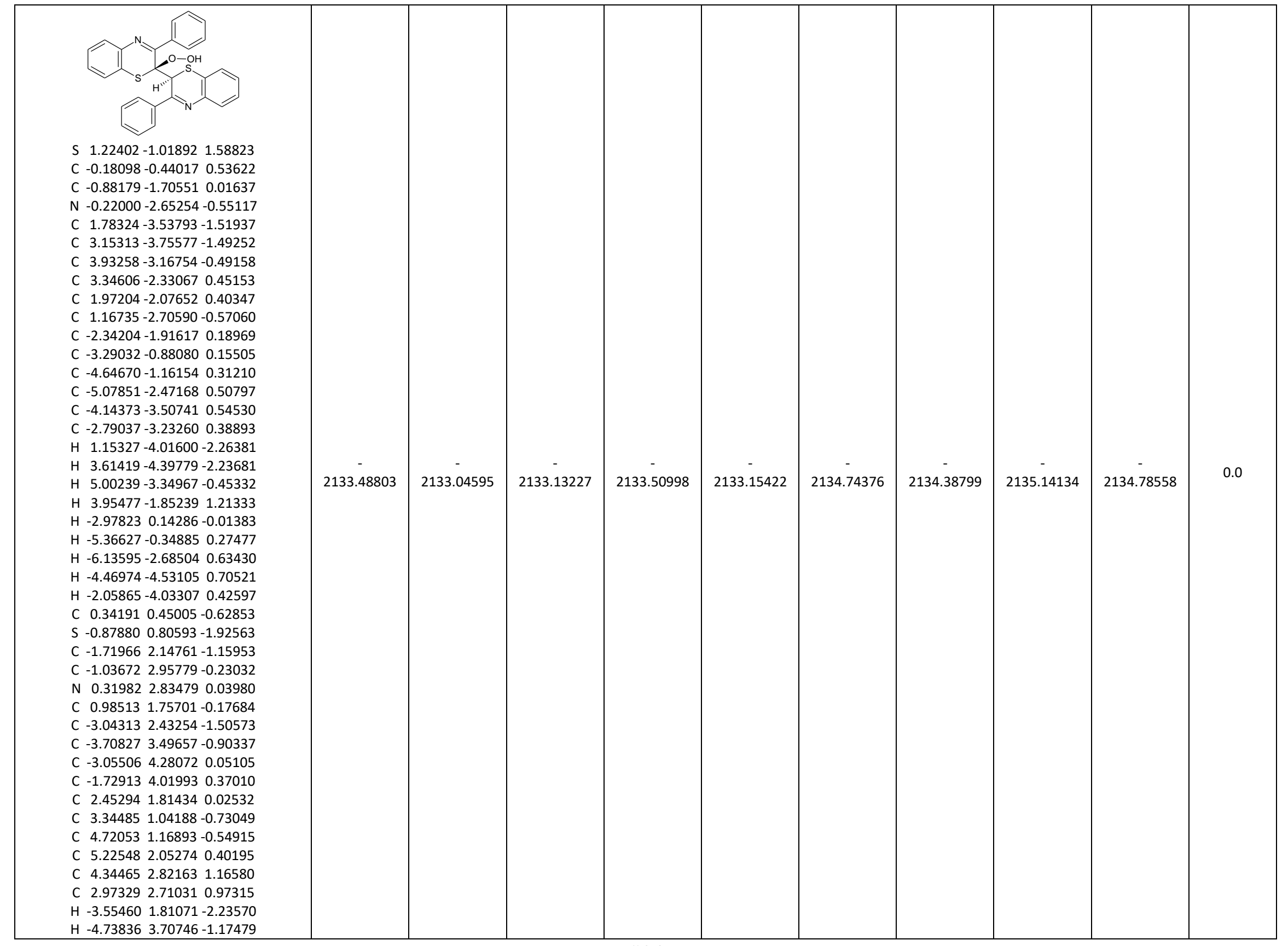




\begin{tabular}{|c|c|c|c|c|c|c|c|c|c|c|}
\hline $\begin{array}{rrrr}H & -3.57569 & 5.10416 & 0.53047 \\
H & -1.19284 & 4.63755 & 1.08484 \\
H & 2.98337 & 0.35325 & -1.48804 \\
H & 5.39709 & 0.57273 & -1.15452 \\
H & 6.29800 & 2.14243 & 0.54866 \\
H & 4.72933 & 3.50853 & 1.91430 \\
H & 2.28350 & 3.30731 & 1.56076 \\
O & -0.98123 & 0.40497 & 1.32302 \\
O & -1.38921 & -0.25019 & 2.52207 \\
H & -2.26909 & -0.58883 & 2.27551 \\
H & 1.08438 & -0.16166 & -1.14588 \\
\end{array}$ & & & & & & & & & & \\
\hline $\begin{array}{llll}\text { S } & 2.12270 & 0.83976 & -1.31425 \\
\mathrm{C} & 0.91239 & 0.35841 & -0.01647 \\
\mathrm{C} & 1.23512 & -1.02598 & 0.58224 \\
\mathrm{~N} & 2.39304 & -1.57871 & 0.61580 \\
\mathrm{C} & 4.75228 & -1.71755 & 0.37450 \\
\mathrm{C} & 5.96482 & -1.29238 & -0.15031 \\
\mathrm{C} & 5.99642 & -0.20128 & -1.02316 \\
\mathrm{C} & 4.81981 & 0.46223 & -1.35600 \\
\mathrm{C} & 3.60125 & 0.03586 & -0.82041 \\
\mathrm{C} & 3.55172 & -1.06566 & 0.04975 \\
\mathrm{C} & 0.14018 & -1.74800 & 1.28551 \\
\mathrm{C} & 0.15592 & -3.15080 & 1.28729 \\
\mathrm{C} & -0.82578 & -3.87273 & 1.95652 \\
\mathrm{C} & -1.83804 & -3.20444 & 2.64797 \\
\mathrm{C} & -1.85676 & -1.81130 & 2.66168 \\
\mathrm{C} & -0.87977 & -1.08498 & 1.98244 \\
\mathrm{H} & 4.69835 & -2.56557 & 1.05110 \\
\mathrm{H} & 6.88273 & -1.80835 & 0.11409 \\
\mathrm{H} & 6.93888 & 0.13756 & -1.44314 \\
\mathrm{H} & 4.84451 & 1.31487 & -2.02940 \\
\mathrm{H} & 0.94495 & -3.66508 & 0.74767 \\
\mathrm{H} & -0.80366 & -4.95880 & 1.93648 \\
\mathrm{H} & -2.60516 & -3.76689 & 3.17278 \\
\mathrm{H} & -2.63339 & -1.28197 & 3.20667 \\
\mathrm{H} & -0.89793 & -0.00179 & 2.01984 \\
\mathrm{C} & -0.42089 & 0.40592 & -0.72490 \\
\mathrm{~S} & -0.66993 & -0.96453 & -1.84214 \\
\mathrm{C} & -2.37653 & -1.28093 & -1.48178 \\
\mathrm{C} & -3.20449 & -0.17175 & -1.27361 \\
\mathrm{~N} & -2.63396 & 1.10568 & -1.22560 \\
\mathrm{C} & -1.39269 & 1.34814 & -0.63935\end{array}$ & $\begin{array}{c}2 \\
- \\
- \\
- \\
2133.48525\end{array}$ & 2133.04290 & $\begin{array}{c}- \\
- \\
2133.13042\end{array}$ & 2133.50845 & 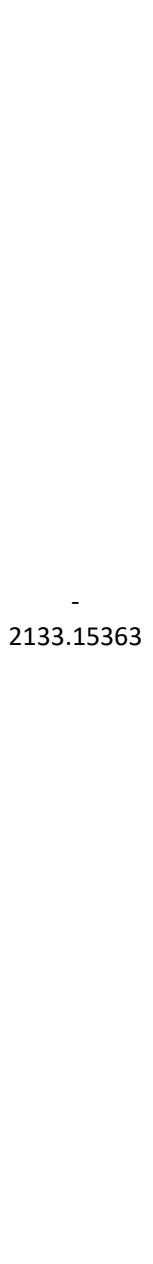 & 2134.74069 & . & 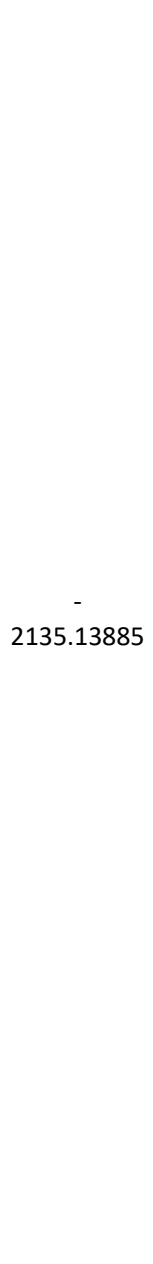 & 2134.78403 & 1.0 \\
\hline
\end{tabular}




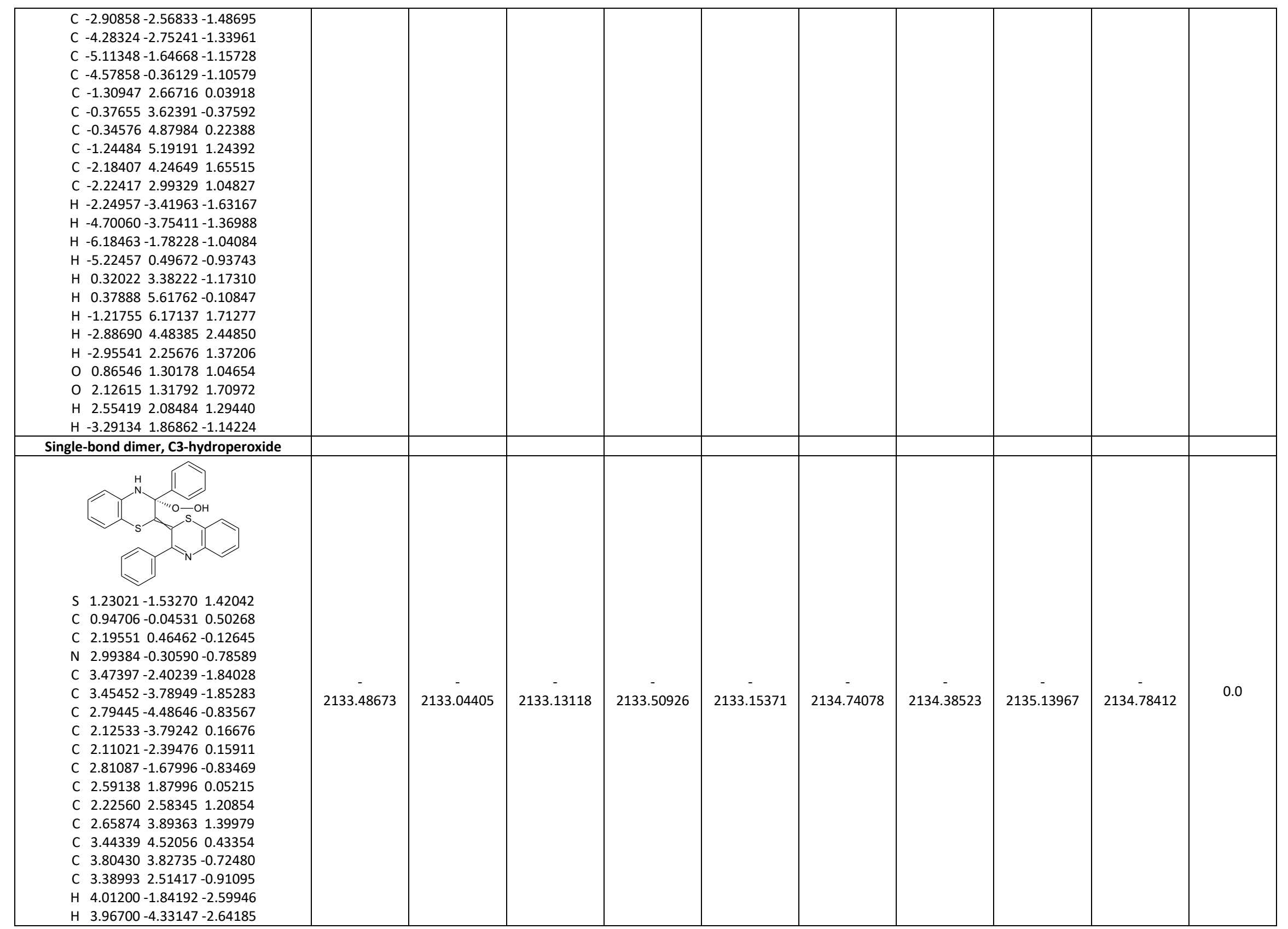




\begin{tabular}{|c|c|c|c|c|c|c|c|c|c|c|}
\hline $\begin{array}{llll}H & 2.79128 & -5.57233 & -0.82881 \\
\text { H } & 1.59316 & -4.33261 & 0.94503 \\
\text { H } & 1.61132 & 2.10033 & 1.96414 \\
\text { H } & 2.37974 & 4.42498 & 2.30511 \\
\text { H } & 3.77199 & 5.54562 & 0.57932 \\
\text { H } & 4.41058 & 4.31415 & -1.48342 \\
\text { H } & 3.66940 & 1.96689 & -1.80567 \\
\text { S } & -0.45649 & 2.07910 & -0.43734 \\
\text { C } & -0.27173 & 0.53157 & 0.41024 \\
\text { C } & -1.48529 & -0.06341 & 1.12446 \\
\text { N } & -2.64179 & 0.84566 & 1.09794 \\
\text { C } & -4.41947 & 1.35097 & -0.50786 \\
\text { C } & -4.81377 & 1.94183 & -1.70514 \\
\text { C } & -3.86899 & 2.52387 & -2.55137 \\
\text { C } & -2.52321 & 2.51827 & -2.19198 \\
\text { C } & -2.13168 & 1.95441 & -0.97761 \\
\text { C } & -3.07451 & 1.36725 & -0.12773 \\
\text { C } & -1.88022 & -1.44273 & 0.60716 \\
\text { C } & -2.54528 & -2.34555 & 1.44415 \\
\text { C } & -3.00176 & -3.56123 & 0.93974 \\
\text { C } & -2.81401 & -3.88091 & -0.40472 \\
\text { C } & -2.16680 & -2.97564 & -1.24372 \\
\text { C } & -1.70152 & -1.76196 & -0.74137 \\
\text { H } & -5.15438 & 0.88476 & 0.14363 \\
\text { H } & -5.86336 & 1.93373 & -1.98358 \\
\text { H } & -4.17500 & 2.97173 & -3.49165 \\
\text { H } & -1.77530 & 2.95846 & -2.84543 \\
\text { H } & -2.69674 & -2.10609 & 2.49202 \\
\text { H } & -3.50518 & -4.26018 & 1.60158 \\
\text { H } & -3.17154 & -4.82900 & -0.79601 \\
\text { H } & -2.01837 & -3.21287 & -2.29327 \\
\text { H } & -1.18934 & -1.06894 & -1.40264 \\
\text { H } & -3.40568 & 0.38689 & 1.58728 \\
\text { O } & -1.19664 & -0.23098 & 2.51101 \\
\text { O } & -0.85700 & 1.03956 & 3.06573 \\
\text { H } & -1.62835 & 1.58028 & 2.79934 \\
\end{array}$ & & & & & & & & & & \\
\hline \multicolumn{11}{|l|}{ Single-bond dimer, dioxolane } \\
\hline $\begin{array}{llll} & \\
\mathrm{S} & -2.14628 & 0.25757 & 0.38775 \\
\mathrm{C} & -0.51503 & -0.43767 & -0.04791 \\
\mathrm{C} & -0.52544 & -1.94088 & 0.26480 \\
\mathrm{~N} & -1.50948 & -2.70604 & -0.04665 \\
\mathrm{C} & -3.53311 & -3.17246 & -1.22804\end{array}$ & $\begin{array}{c}- \\
2133.50577\end{array}$ & $\begin{array}{c}- \\
2133.06276\end{array}$ & $\begin{array}{c}- \\
2133.14809\end{array}$ & $\begin{array}{c}- \\
2133.52715\end{array}$ & $\begin{array}{c}- \\
2133.16947\end{array}$ & $\begin{array}{c}- \\
2134.75632\end{array}$ & $\begin{array}{c}- \\
2134.39863\end{array}$ & $\begin{array}{c}- \\
2135.15244\end{array}$ & $\begin{array}{c}- \\
2134.79476\end{array}$ & 0.0 \\
\hline
\end{tabular}




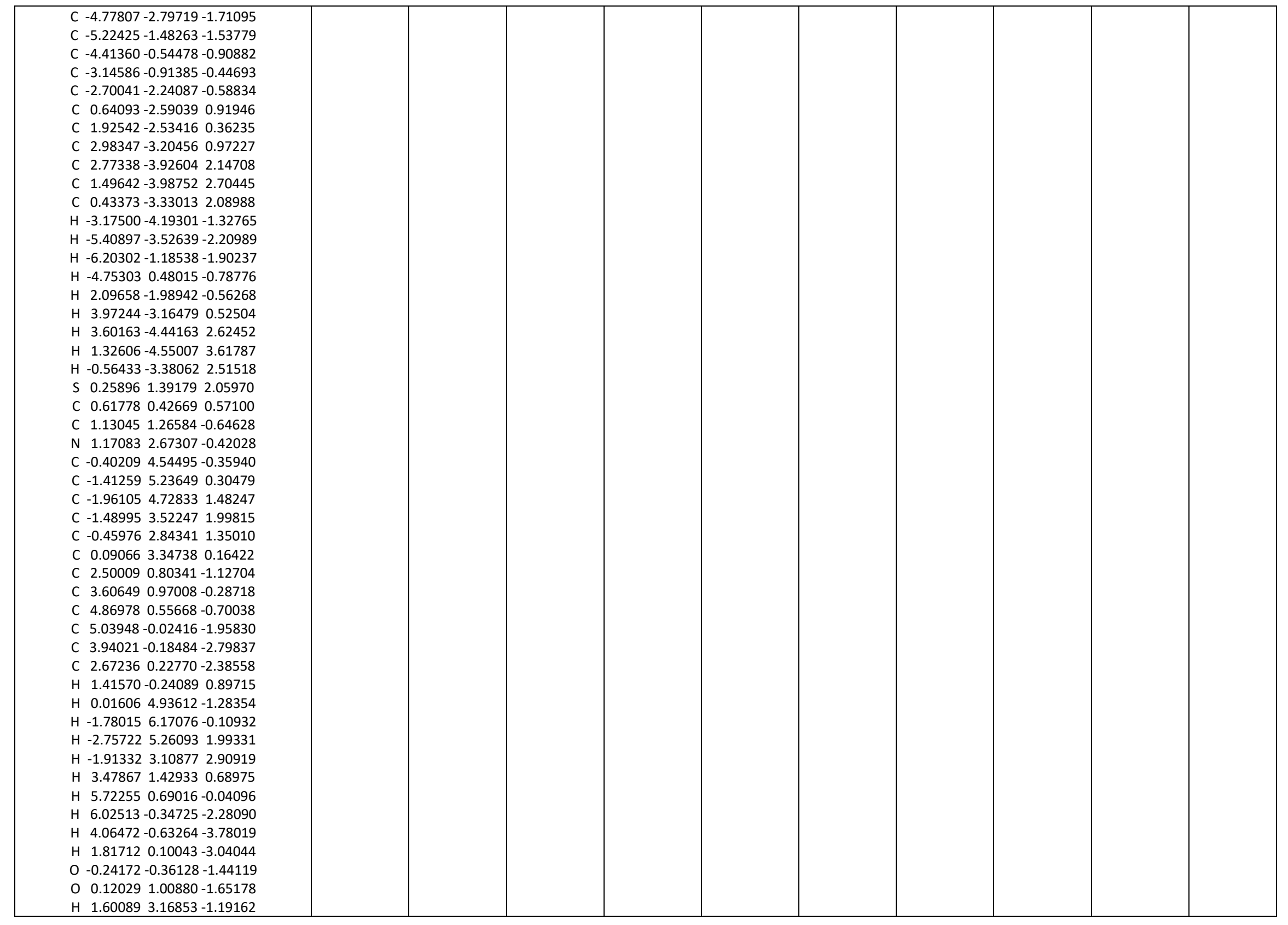




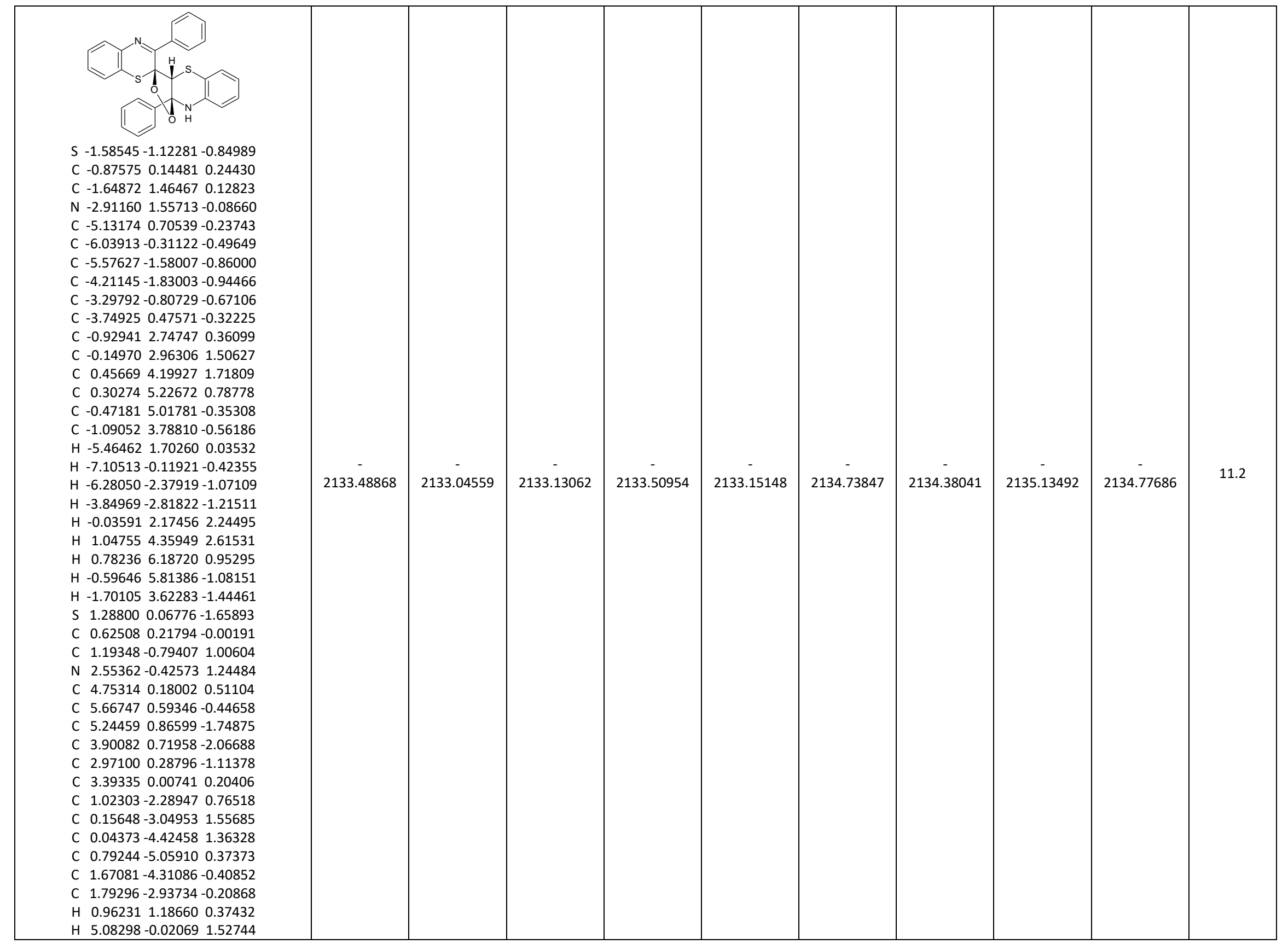




\begin{tabular}{|c|c|c|c|c|c|c|c|c|c|c|}
\hline $\begin{array}{lrrrr}H & 6.71130 & 0.71066 & -0.17121 \\
H & 5.94896 & 1.19821 & -2.50460 \\
H & 3.55324 & 0.93937 & -3.07302 \\
H & -0.43463 & -2.56314 & 2.32427 \\
H & -0.63386 & -4.99958 & 1.98812 \\
H & 0.70116 & -6.13066 & 0.22023 \\
H & 2.27222 & -4.79620 & -1.17187 \\
H & 2.50201 & -2.37802 & -0.80972 \\
\text { O } & -0.93583 & -0.24341 & 1.63966 \\
\text { O } & 0.42101 & -0.37786 & 2.14871 \\
H & 3.02467 & -1.03015 & 1.90643 \\
\end{array}$ & & & & & & & & & & \\
\hline 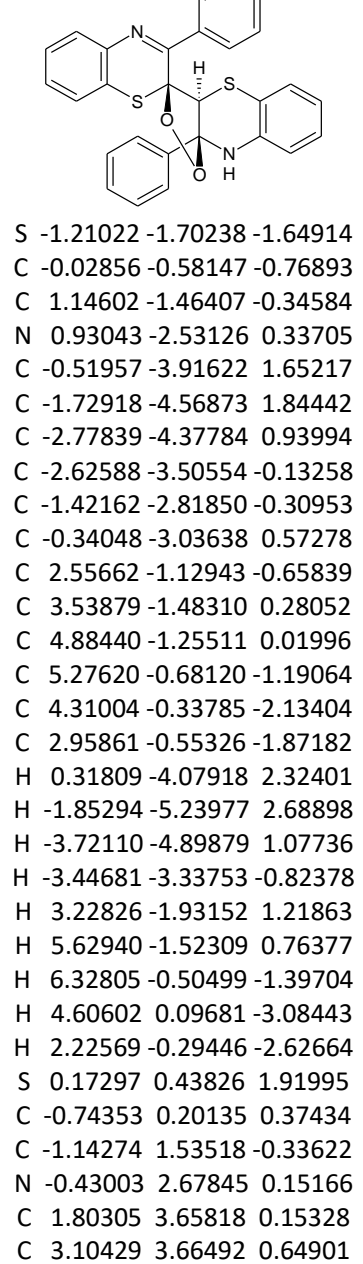 & 2133.50482 & $\begin{array}{c}- \\
2133.06177\end{array}$ & 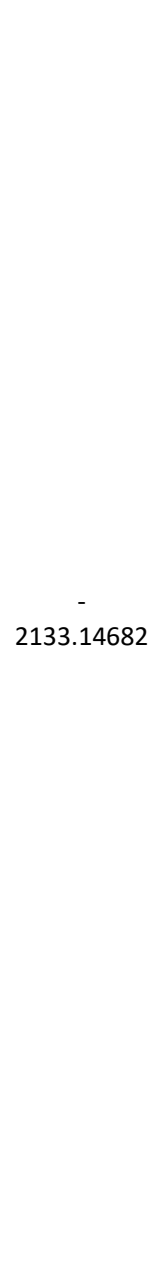 & 更 & 西 & 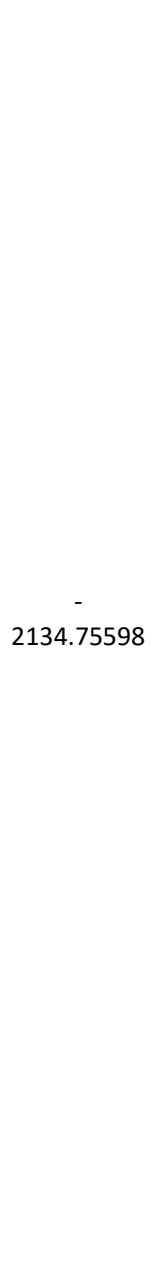 & . & 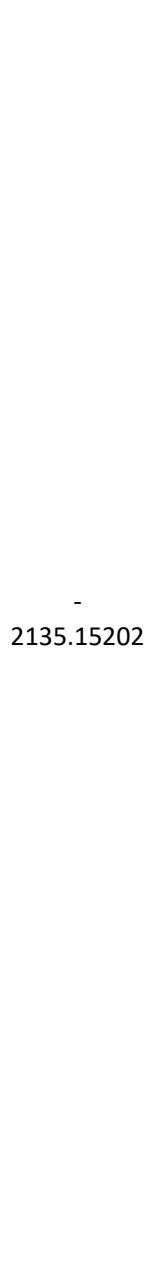 & 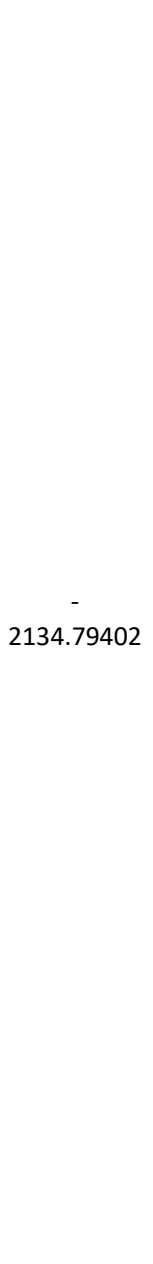 & 0.5 \\
\hline
\end{tabular}




\begin{tabular}{|c|c|c|c|c|c|c|c|c|c|c|}
\hline $\begin{array}{lrrrr}\text { C } & 3.54217 & 2.65361 & 1.50473 \\
\text { C } & 2.66750 & 1.62987 & 1.86437 \\
\text { C } & 1.35672 & 1.63502 & 1.39171 \\
\text { C } & 0.91162 & 2.64910 & 0.53187 \\
\text { C } & -2.63929 & 1.81359 & -0.30409 \\
\text { C } & -3.50218 & 1.22712 & -1.23589 \\
\text { C } & -4.87296 & 1.46679 & -1.16865 \\
\text { C } & -5.39761 & 2.28273 & -0.16697 \\
\text { C } & -4.54136 & 2.86148 & 0.76765 \\
\text { C } & -3.16812 & 2.63158 & 0.69874 \\
\text { H } & -1.64232 & -0.33468 & 0.68296 \\
\text { H } & 1.46671 & 4.44397 & -0.51869 \\
\text { H } & 3.78232 & 4.46065 & 0.35423 \\
\text { H } & 4.56016 & 2.65375 & 1.88194 \\
\text { H } & 2.99643 & 0.82752 & 2.51850 \\
H & -3.10170 & 0.58339 & -2.01096 \\
H & -5.53195 & 1.01256 & -1.90308 \\
\text { H } & -6.46706 & 2.46589 & -0.11566 \\
\text { H } & -4.93926 & 3.49661 & 1.55387 \\
\text { H } & -2.50220 & 3.08564 & 1.42529 \\
\text { O } & 0.34776 & 0.42570 & -1.66758 \\
\text { O } & -0.82154 & 1.26105 & -1.72260 \\
\text { H } & -0.67991 & 3.50807 & -0.37346 \\
\end{array}$ & & & & & & & & & & \\
\hline 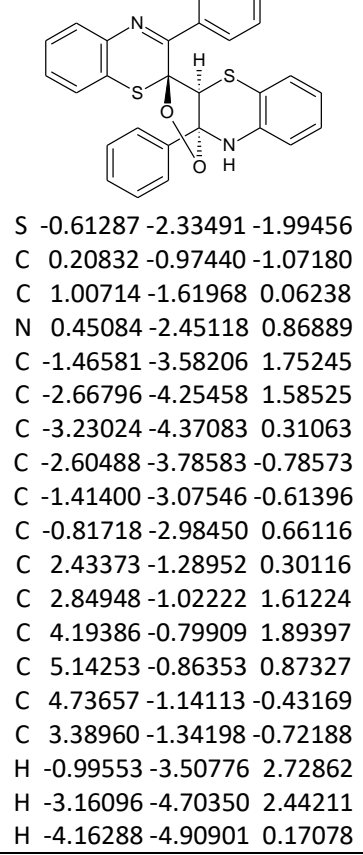 & 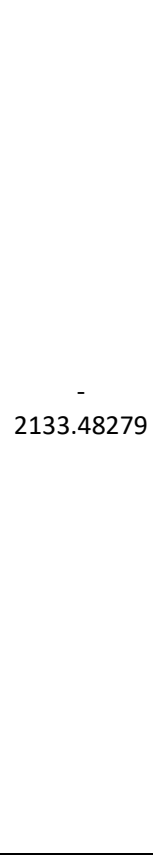 & 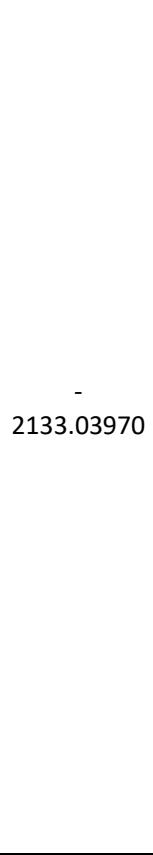 & $\begin{array}{c}- \\
2133.12349\end{array}$ & 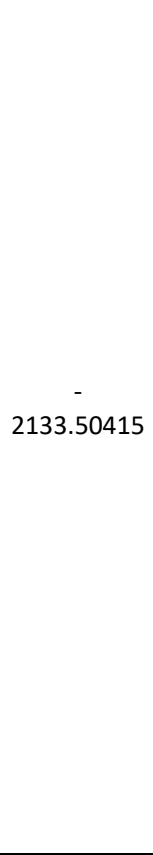 & 更 & 更 & $\begin{array}{c}- \\
2134.37791\end{array}$ & 更 & $\begin{array}{c}- \\
2134.77446\end{array}$ & 12.7 \\
\hline
\end{tabular}




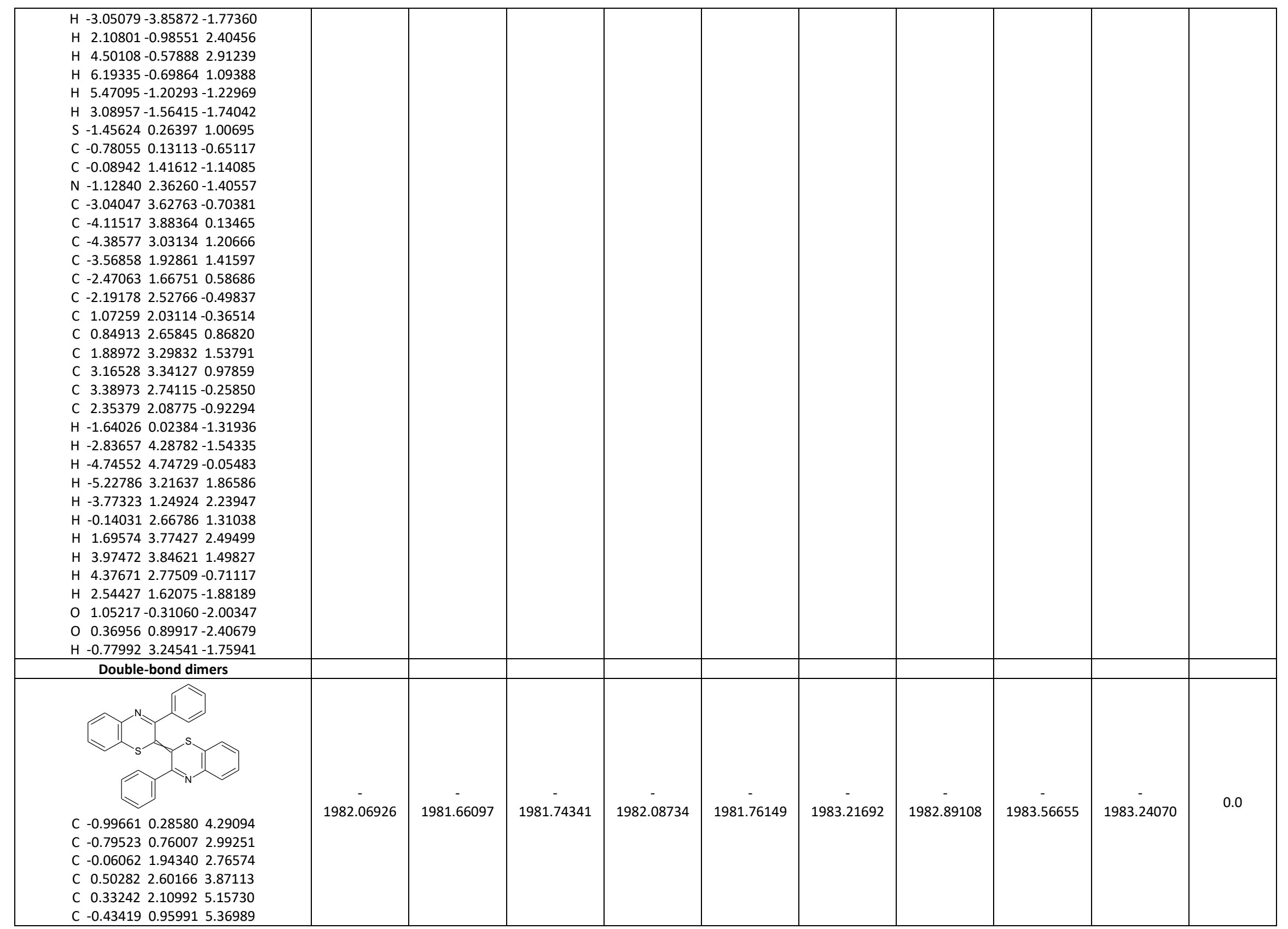




\begin{tabular}{|c|c|c|c|c|c|c|c|c|c|c|}
\hline $\begin{array}{lrrr}\text { N } & 0.01510 & 2.56088 & 1.52588 \\
\text { C } & -0.15936 & 1.91126 & 0.42329 \\
\text { C } & -0.32068 & 0.43393 & 0.40949 \\
\text { S } & -1.48612 & -0.10834 & 1.62205 \\
\text { C } & 0.32068 & -0.43393 & -0.40949 \\
\text { C } & 0.15936 & -1.91126 & -0.42329 \\
\text { N } & -0.01510 & -2.56088 & -1.52588 \\
\text { C } & 0.06062 & -1.94340 & -2.76574 \\
\text { C } & 0.79523 & -0.76007 & -2.99251 \\
\text { S } & 1.48612 & 0.10834 & -1.62205 \\
\text { C } & 0.99661 & -0.28580 & -4.29094 \\
\text { C } & 0.43419 & -0.95991 & -5.36989 \\
\text { C } & -0.33242 & -2.10992 & -5.15730 \\
\text { C } & -0.50282 & -2.60166 & -3.87113 \\
\text { C } & 0.27665 & -2.69689 & 0.82766 \\
\text { C } & -0.27665 & 2.69689 & -0.82766 \\
\text { H } & 1.06536 & 3.51284 & 3.68915 \\
\text { H } & 0.78052 & 2.62952 & 5.99879 \\
\text { H } & -0.58624 & 0.58122 & 6.37615 \\
\text { H } & -1.57356 & -0.62066 & 4.45109 \\
\text { H } & -1.06536 & -3.51284 & -3.68915 \\
\text { H } & -0.78052 & -2.62952 & -5.99879 \\
\text { H } & 0.58624 & -0.58122 & -6.37615 \\
\text { H } & 1.57356 & 0.62066 & -4.45109 \\
\text { C } & 1.09922 & -2.26014 & 1.87392 \\
\text { C } & 1.26266 & -3.04220 & 3.01357 \\
\text { C } & 0.59567 & -4.26190 & 3.12830 \\
\text { C } & -0.22894 & -4.70075 & 2.09086 \\
\text { C } & -0.38115 & -3.92846 & 0.94395 \\
\text { H } & 1.62132 & -1.31090 & 1.79134 \\
\text { H } & 1.91192 & -2.69784 & 3.81342 \\
\text { H } & 0.71739 & -4.86778 & 4.02166 \\
\text { H } & -0.75509 & -5.64727 & 2.17662 \\
\text { H } & -1.01714 & -4.26493 & 0.13126 \\
\text { C } & 0.38115 & 3.92846 & -0.94395 \\
\text { C } & 0.22894 & 4.70075 & -2.09086 \\
\text { C } & -0.59567 & 4.26190 & -3.12830 \\
\text { C } & -1.26266 & 3.04220 & -3.01357 \\
\text { C } & -1.09922 & 2.26014 & -1.87392 \\
\text { H } & 1.01714 & 4.26493 & -0.13126 \\
\text { H } & 0.75509 & 5.64727 & -2.17662 \\
\text { H } & -0.71739 & 4.86778 & -4.02166 \\
\text { H } & -1.91192 & 2.69784 & -3.81342 \\
\text { H } & -1.62132 & 1.31090 & -1.79134 \\
\end{array}$ & & & & & & & 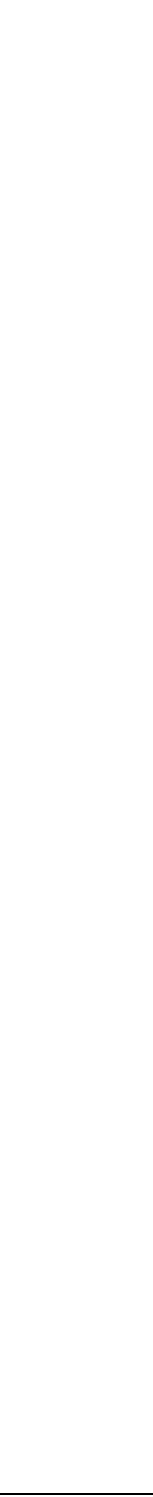 & & & \\
\hline \multicolumn{11}{|l|}{ Oxygen species } \\
\hline \begin{tabular}{cccc} 
& \multicolumn{3}{c}{$\mathrm{O}_{2}$} \\
$\mathrm{O}$ & 0.00000 & 0.00000 & 0.60130 \\
0 & 0.000000 & 0.00000 & -0.60130 \\
\end{tabular} & -150.17445 & -150.16722 & -150.19049 & -150.17424 & -150.19028 & -150.26380 & -150.27984 & -150.31351 & -150.32955 & 0.0 \\
\hline $\begin{array}{lll} & \mathrm{H}_{2} \mathrm{O}_{2} \\
0 & 0.00000 & 0.71450-0.06520\end{array}$ & -151.40433 & -151.37324 & -151.39898 & -151.41318 & -151.40783 & -151.50645 & -151.50109 & -151.55778 & -151.55243 & 0.0 \\
\hline
\end{tabular}




\begin{tabular}{|c|c|c|c|c|c|c|c|c|c|c|c|}
\hline $\begin{array}{rr}\text { O } & 0.00000 \\
\text { H } & -0.74458 \\
\text { H } & 0.74458\end{array}$ & $\begin{array}{cc}-0.71450 & -0.06520 \\
-0.91370 & 0.52162 \\
0.91370 & 0.52162\end{array}$ & & & & & & & & & & \\
\hline $\begin{array}{ll} & \\
\text { O } & 0.00000 \\
\text { H } & 0.00000 \\
\text { H } & 0.00000\end{array}$ & $\begin{array}{lll}\mathrm{H}_{2} \mathrm{O} & \\
0.00000 & 0.11750 \\
0.76275 & -0.47000 \\
-0.76275 & -0.47000\end{array}$ & -76.35719 & -76.33188 & -76.35331 & -76.36339 & -76.35950 & & & & & 0.0 \\
\hline $\begin{array}{ll} & \\
& 0.05522 \\
\text { O } & 0.05522 \\
\text { H } & -0.88355 \\
\end{array}$ & $\begin{array}{ll}\text { HOO. } & \\
0.71139 & 0.00000 \\
-0.60193 & 0.00000 \\
-0.87569 & 0.00000 \\
\end{array}$ & -150.76380 & -150.74551 & -150.77146 & -150.76970 & -150.77736 & -150.85860 & -150.86626 & -150.90856 & -150.91622 & 0.0 \\
\hline $\begin{array}{ll} & \\
& 0.00000 \\
\text { H } & 0.00000 \\
\end{array}$ & $\begin{array}{ll}\text { HO. } \\
0.00000 & 0.10848 \\
0.00000 & -0.86783\end{array}$ & -75.66227 & -75.65038 & -75.67062 & -75.66470 & -75.67305 & & & & & 0.0 \\
\hline
\end{tabular}


Table S5. Monoprotonated forms in methanol. For each chemical species, values are reported for the most stable conformer / geometric isomer identified. $G_{\text {RRHo,calc }}[M 062 X / 6-311++G(2 d, 2 p) / S M D]=G_{\text {el }}[M 062 X / 6-311++G(2 d, 2 p) / S M D / / P B E 0 / 6-31+G(d, p) / P C M]-G_{\text {el }}[P B E 0 / 6-31+G(d, p)$ $/ \mathrm{PCM}]+G_{\mathrm{RRHO}}[\mathrm{PBEO} / 6-31+\mathrm{G}(\mathrm{d}, \mathrm{p}) / \mathrm{PCM}]$; and likewise for $G_{\mathrm{RRHO}, \text { calc }}[\mathrm{PBEO} / 6-31+\mathrm{G}(\mathrm{d}, \mathrm{p}) / \mathrm{SMD}]$ and for $G_{\mathrm{RRHO}, \text { calc }}[\mathrm{MO62X} / 6-31+\mathrm{G}(\mathrm{d}, \mathrm{p}) / \mathrm{SMD}]$.

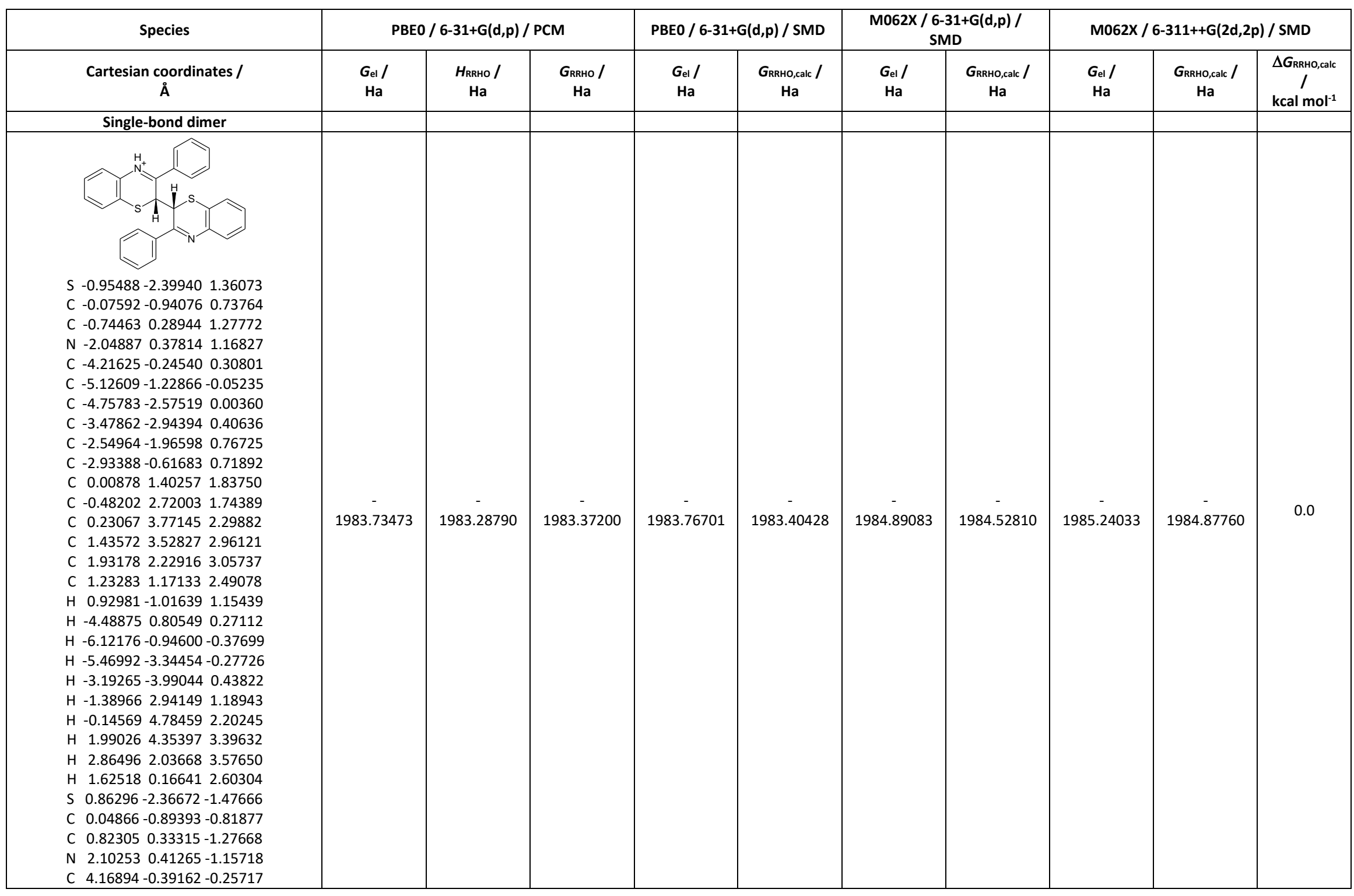




\begin{tabular}{|c|c|c|c|c|c|c|c|c|c|c|}
\hline $\begin{array}{lrrr}\text { C } & 5.03805 & -1.42273 & 0.06968 \\
\text { C } & 4.64103 & -2.75209 & -0.10361 \\
\text { C } & 3.36406 & -3.04418 & -0.57125 \\
\text { C } & 2.47633 & -2.00856 & -0.87488 \\
\text { C } & 2.87789 & -0.66172 & -0.73977 \\
\text { C } & 0.09178 & 1.50240 & -1.81499 \\
\text { C } & 0.73058 & 2.75462 & -1.83650 \\
\text { C } & 0.08006 & 3.87481 & -2.33510 \\
\text { C } & -1.22227 & 3.76983 & -2.83030 \\
\text { C } & -1.86395 & 2.53414 & -2.82243 \\
\text { C } & -1.21548 & 1.40951 & -2.31562 \\
\text { H } & -0.95115 & -0.91453 & -1.25365 \\
\text { H } & 4.46824 & 0.64767 & -0.15722 \\
\text { H } & 6.03086 & -1.19471 & 0.44529 \\
\text { H } & 5.32301 & -3.56265 & 0.13460 \\
\text { H } & 3.04597 & -4.07632 & -0.68824 \\
\text { H } & 1.74010 & 2.83049 & -1.44672 \\
\text { H } & 0.58655 & 4.83565 & -2.33469 \\
\text { H } & -1.73080 & 4.64661 & -3.22048 \\
\text { H } & -2.87155 & 2.43830 & -3.21574 \\
\text { H } & -1.73910 & 0.45945 & -2.35117 \\
\text { H } & -2.49067 & 1.22649 & 1.51219\end{array}$ & & & & & & & & & & \\
\hline 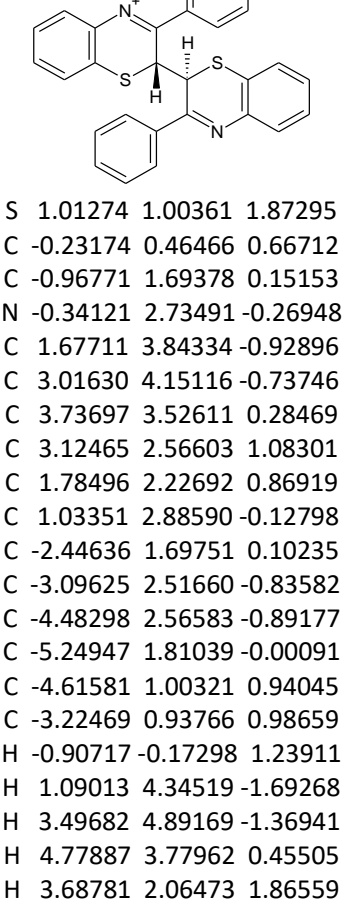 & $\begin{array}{c}- \\
- \\
- \\
1983.73492\end{array}$ & $\begin{array}{c}- \\
1983.28791\end{array}$ & $\begin{array}{c}- \\
- \\
- \\
1983.37071\end{array}$ & $\begin{array}{c}- \\
- \\
- \\
1983.76576\end{array}$ & $\begin{array}{c}- \\
- \\
- \\
1983.40155\end{array}$ & $\begin{array}{c}- \\
- \\
- \\
1984.89051\end{array}$ & $\begin{array}{c}- \\
- \\
-1984.52630\end{array}$ & $\begin{array}{c}2.20 \\
- \\
- \\
-1985.24064\end{array}$ & $\begin{array}{c}- \\
- \\
- \\
1984.87643\end{array}$ & 0.7 \\
\hline
\end{tabular}




\begin{tabular}{|c|c|c|c|c|c|c|c|c|c|c|}
\hline $\begin{array}{llll}H & -2.49507 & 3.10258 & -1.52330 \\
\text { H } & -4.96983 & 3.19365 & -1.63247 \\
\text { H } & -6.33401 & 1.85232 & -0.04223 \\
\text { H } & -5.20253 & 0.41974 & 1.64377 \\
\text { H } & -2.76185 & 0.31247 & 1.74416 \\
\text { S } & -0.83164 & -0.85422 & -1.75648 \\
\text { C } & 0.38390 & -0.34908 & -0.49636 \\
\text { C } & 1.04255 & -1.63175 & -0.06598 \\
\text { N } & 0.25791 & -2.56448 & 0.43428 \\
\text { C } & -1.85851 & -3.51580 & 1.11681 \\
\text { C } & -3.21167 & -3.70550 & 0.88163 \\
\text { C } & -3.84057 & -3.03317 & -0.17042 \\
\text { C } & -3.12781 & -2.15009 & -0.97297 \\
\text { C } & -1.76757 & -1.93724 & -0.73968 \\
\text { C } & -1.13658 & -2.64352 & 0.29757 \\
\text { C } & 2.46431 & -1.89208 & -0.20014 \\
\text { C } & 2.94018 & -3.21693 & -0.28480 \\
\text { C } & 4.29970 & -3.46602 & -0.38970 \\
\text { C } & 5.20498 & -2.40309 & -0.41801 \\
\text { C } & 4.74456 & -1.08804 & -0.35554 \\
\text { C } & 3.38456 & -0.82957 & -0.25679 \\
\text { H } & 1.10129 & 0.26356 & -1.04552 \\
\text { H } & -1.35415 & -4.04076 & 1.92275 \\
\text { H } & -3.77698 & -4.38111 & 1.51463 \\
\text { H } & -4.89883 & -3.18606 & -0.35648 \\
\text { H } & -3.62481 & -1.61045 & -1.77284 \\
\text { H } & 2.25182 & -4.05598 & -0.32355 \\
\text { H } & 4.65428 & -4.48849 & -0.46782 \\
\text { H } & 6.26905 & -2.60123 & -0.50329 \\
\text { H } & 5.44638 & -0.26087 & -0.38381 \\
\text { H } & 3.04873 & 0.19925 & -0.19339 \\
\text { H } & 0.70234 & -3.37353 & 0.85861\end{array}$ & & & & 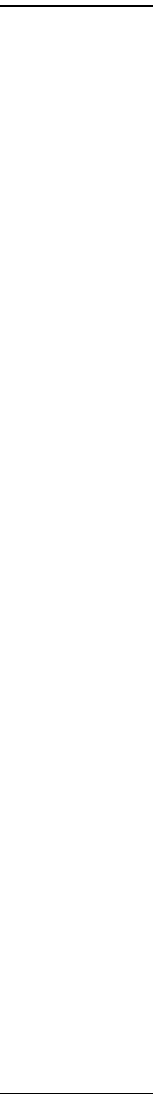 & 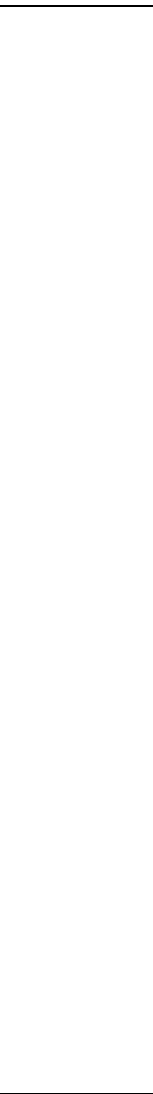 & 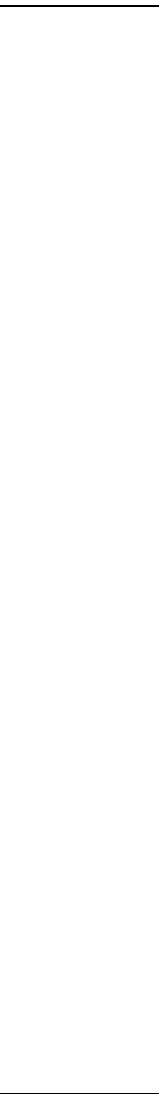 & & & & \\
\hline 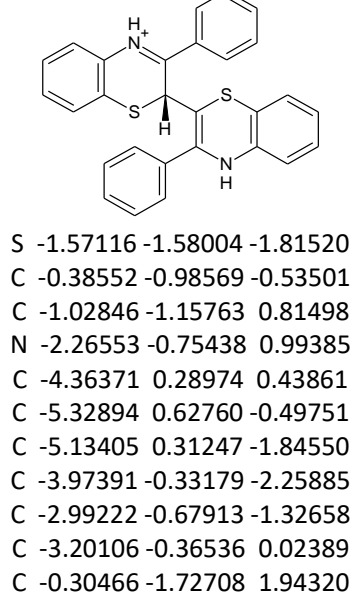 & $\begin{array}{c}- \\
- \\
- \\
1983.73393\end{array}$ & $\begin{array}{c}- \\
- \\
- \\
1983.28656\end{array}$ & $\begin{array}{c}- \\
1983.37001\end{array}$ & 更 & $\begin{array}{c}- \\
1983.40107\end{array}$ & $\stackrel{-}{-}$ & $\begin{array}{c}- \\
1984.52250\end{array}$ & $\begin{array}{c}- \\
1985.23695\end{array}$ & $\begin{array}{c}- \\
- \\
1984.87302\end{array}$ & 2.9 \\
\hline
\end{tabular}




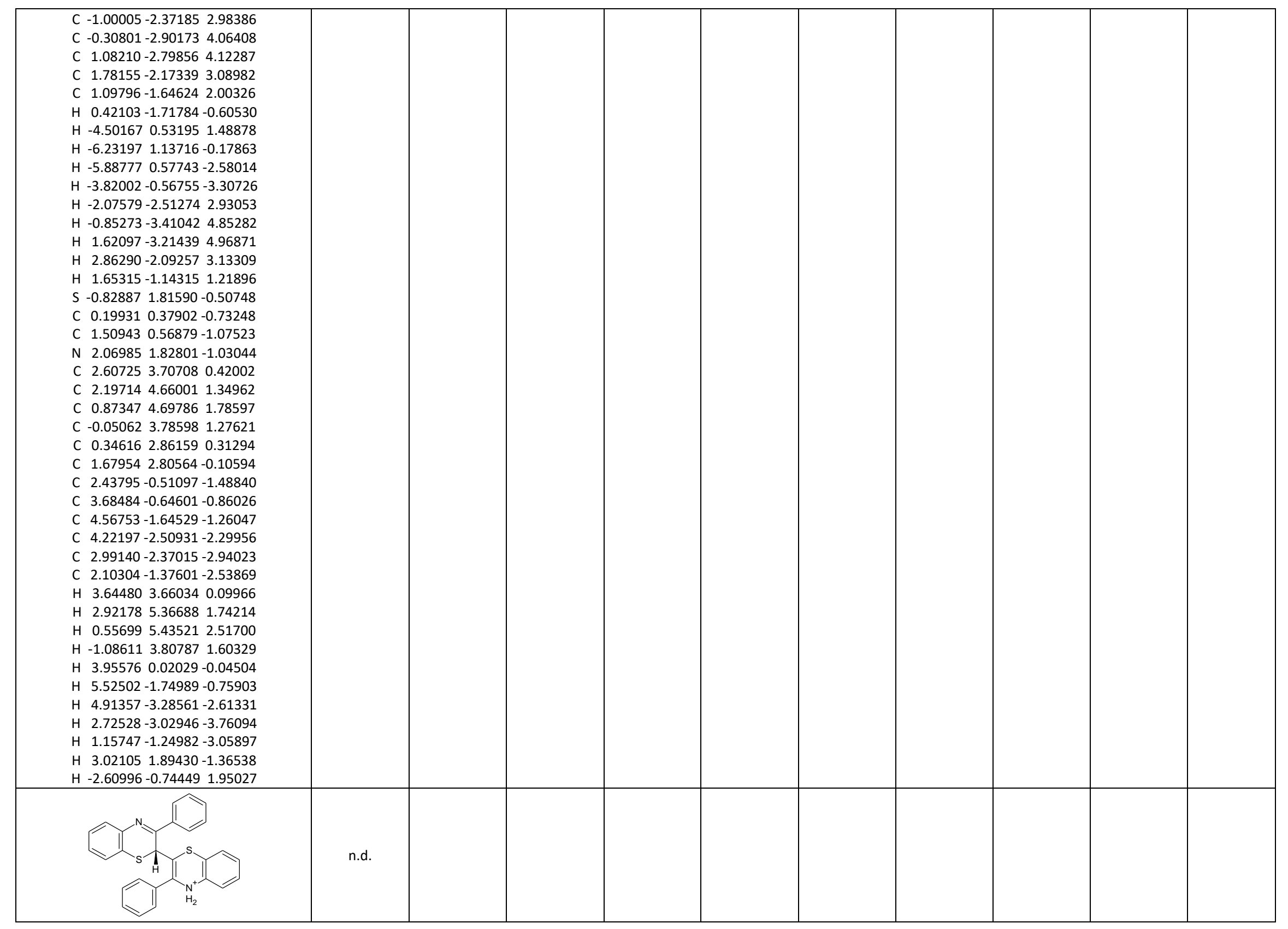




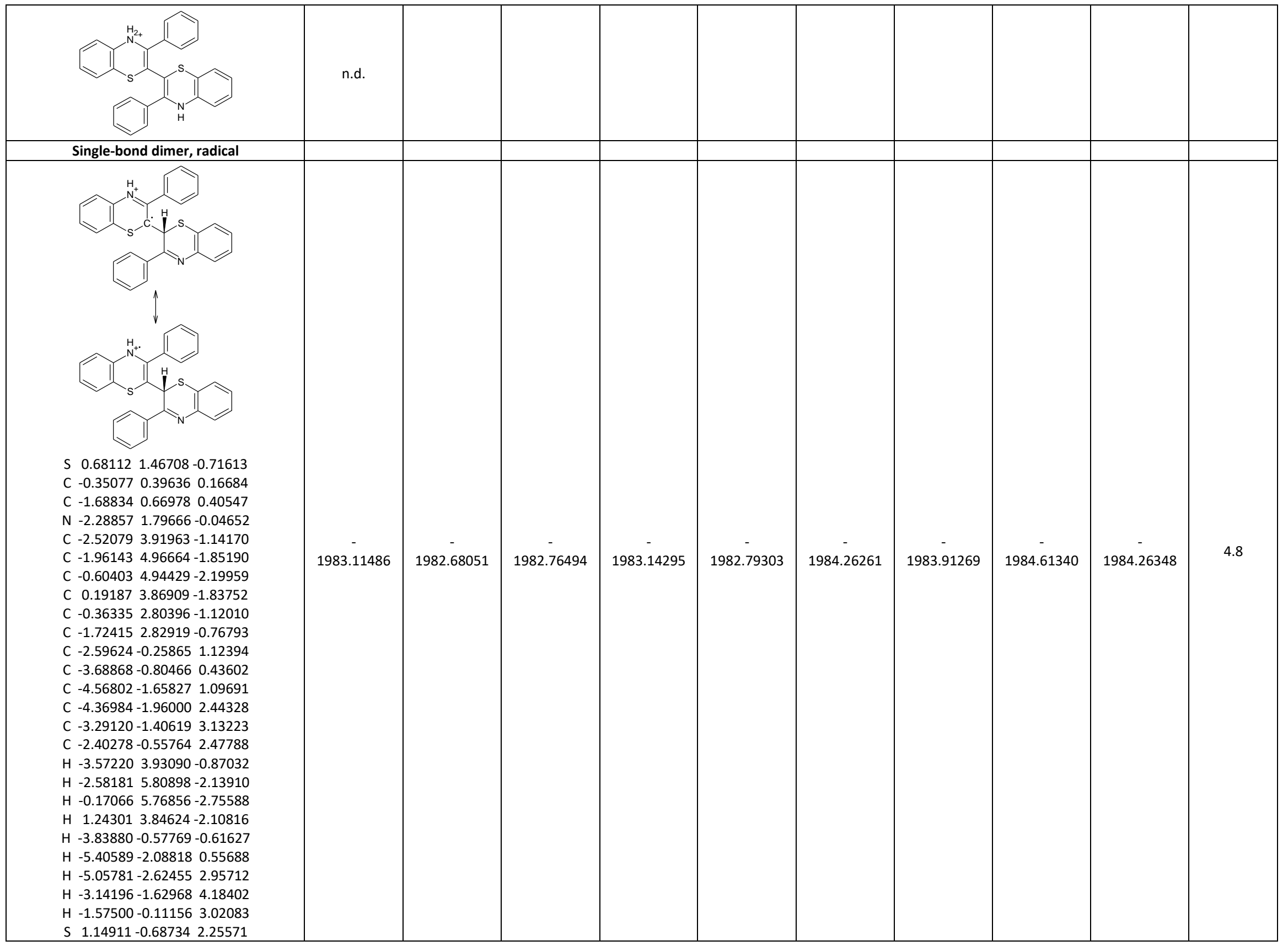




\begin{tabular}{|c|c|c|c|c|c|c|c|c|c|c|}
\hline $\begin{array}{lllll}C & 0.29782 & -0.87506 & 0.64538 \\
\text { C } & 1.25956 & -1.51750 & -0.35773 \\
\text { N } & 2.50496 & -1.20900 & -0.44476 \\
\text { C } & 4.36550 & 0.21888 & -0.00421 \\
\text { C } & 5.09219 & 1.06242 & 0.82432 \\
\text { C } & 4.61149 & 1.37037 & 2.10042 \\
\text { C } & 3.39489 & 0.85316 & 2.53314 \\
\text { C } & 2.65315 & 0.01739 & 1.69372 \\
\text { C } & 3.13981 & -0.32153 & 0.41422 \\
\text { C } & 0.71846 & -2.53376 & -1.28584 \\
\text { C } & -0.65959 & -2.73863 & -1.44823 \\
\text { C } & -1.13477 & -3.68822 & -2.34999 \\
\text { C } & -0.24229 & -4.45514 & -3.09493 \\
\text { C } & 1.13275 & -4.26364 & -2.93764 \\
\text { C } & 1.60820 & -3.31192 & -2.04592 \\
\text { H } & -0.49557 & -1.58731 & 0.87707 \\
\text { H } & 4.72821 & -0.04761 & -0.99255 \\
\text { H } & 6.03757 & 1.47331 & 0.48373 \\
\text { H } & 5.18107 & 2.02036 & 2.75765 \\
\text { H } & 3.01331 & 1.10101 & 3.51954 \\
\text { H } & -1.38355 & -2.15559 & -0.88777 \\
\text { H } & -2.20540 & -3.82722 & -2.46638 \\
\text { H } & -0.61367 & -5.20011 & -3.79257 \\
\text { H } & 1.83469 & -4.86168 & -3.51144 \\
\text { H } & 2.67441 & -3.15706 & -1.91932 \\
\text { H } & -3.26497 & 1.90717 & 0.20545 \\
\end{array}$ & 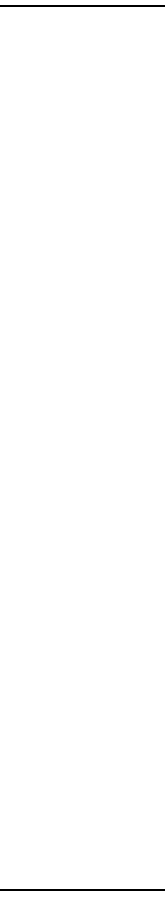 & & 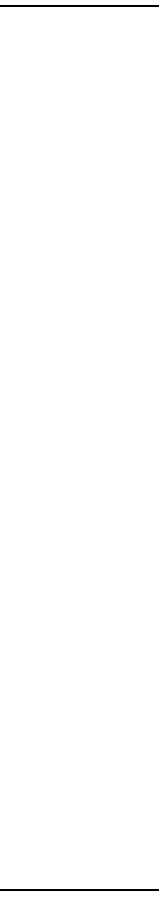 & 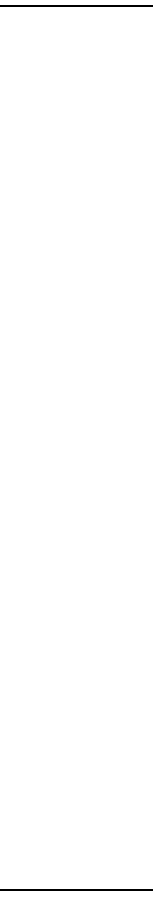 & 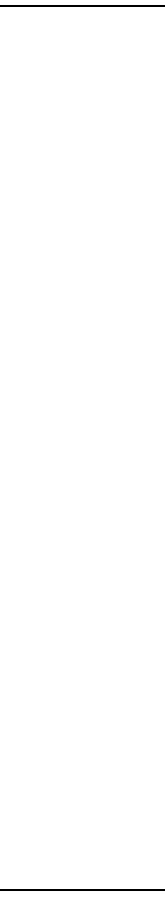 & 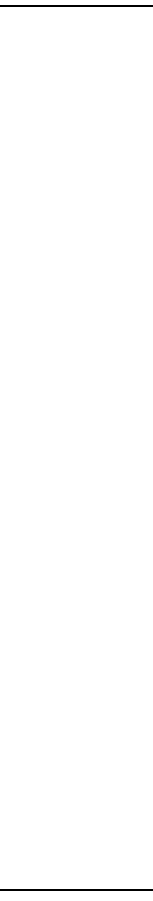 & & & & \\
\hline $\begin{array}{lrll} & \\
\mathrm{S} & -1.22412 & 1.42628 & 0.06639 \\
\mathrm{C} & 0.12237 & 0.47012 & -0.46520 \\
\mathrm{C} & 1.40369 & 1.03544 & -0.65451 \\
\mathrm{~N} & 1.77180 & 2.26308 & -0.31815 \\
\mathrm{C} & 1.44615 & 4.41062 & 0.63504 \\
\mathrm{C} & 0.65034 & 5.39156 & 1.19761 \\
\mathrm{C} & -0.71757 & 5.15890 & 1.39841 \\
\mathrm{C} & -1.27606 & 3.93983 & 1.04130\end{array}$ & 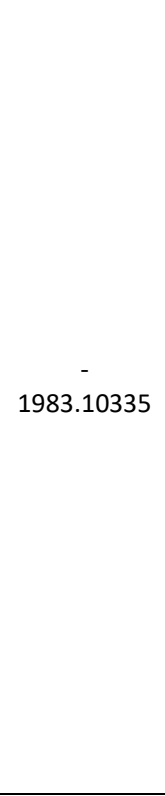 & 更 & 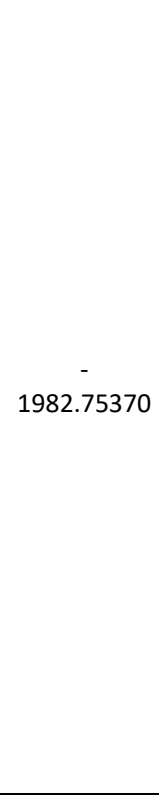 & 列 & $\begin{array}{c}- \\
1982.78368\end{array}$ & 列 & $\begin{array}{c}- \\
1983.90171\end{array}$ & $\begin{array}{c}- \\
1984.60140\end{array}$ & $\begin{array}{c}- \\
1984.25175\end{array}$ & 12.2 \\
\hline
\end{tabular}




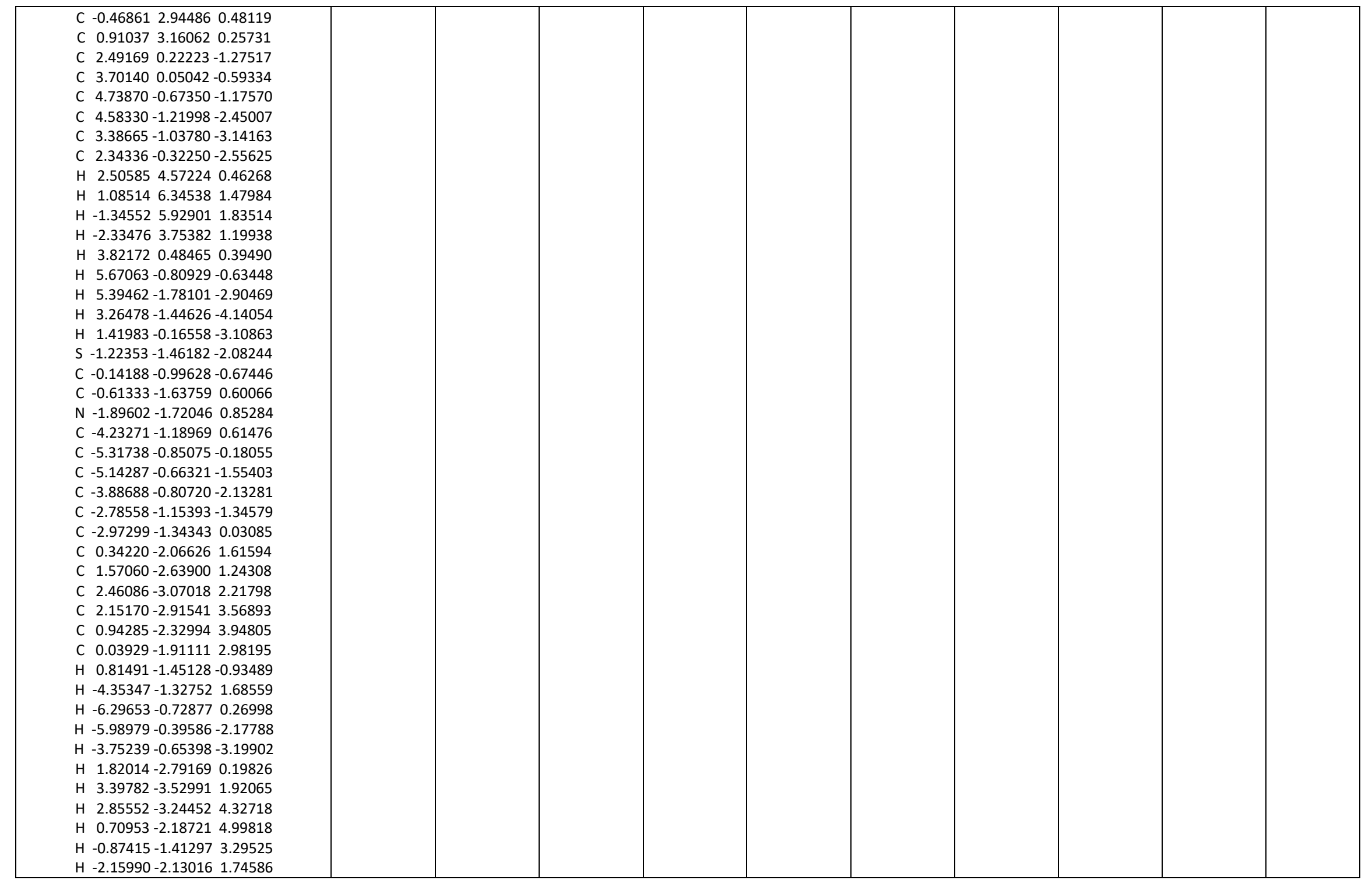




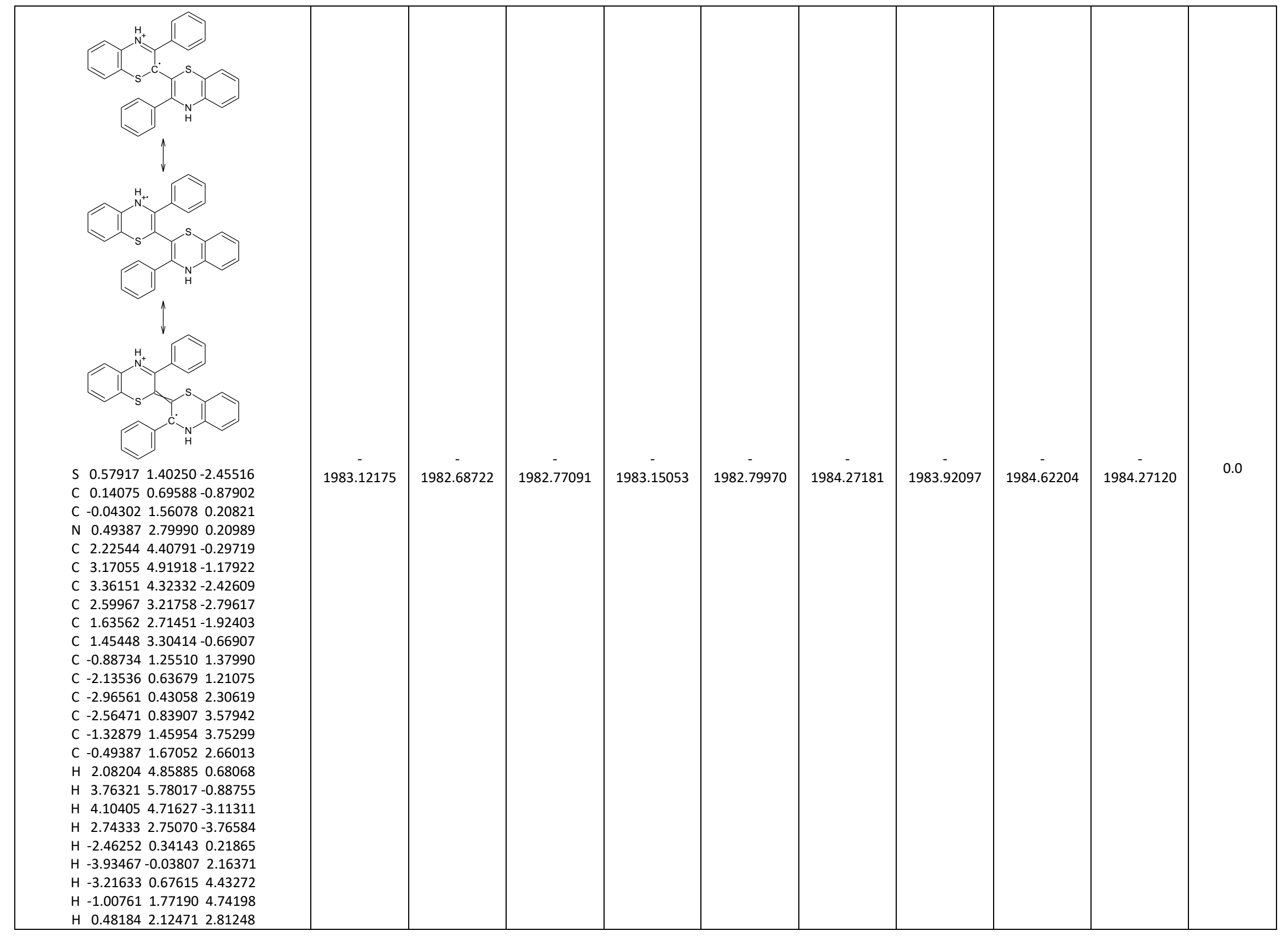




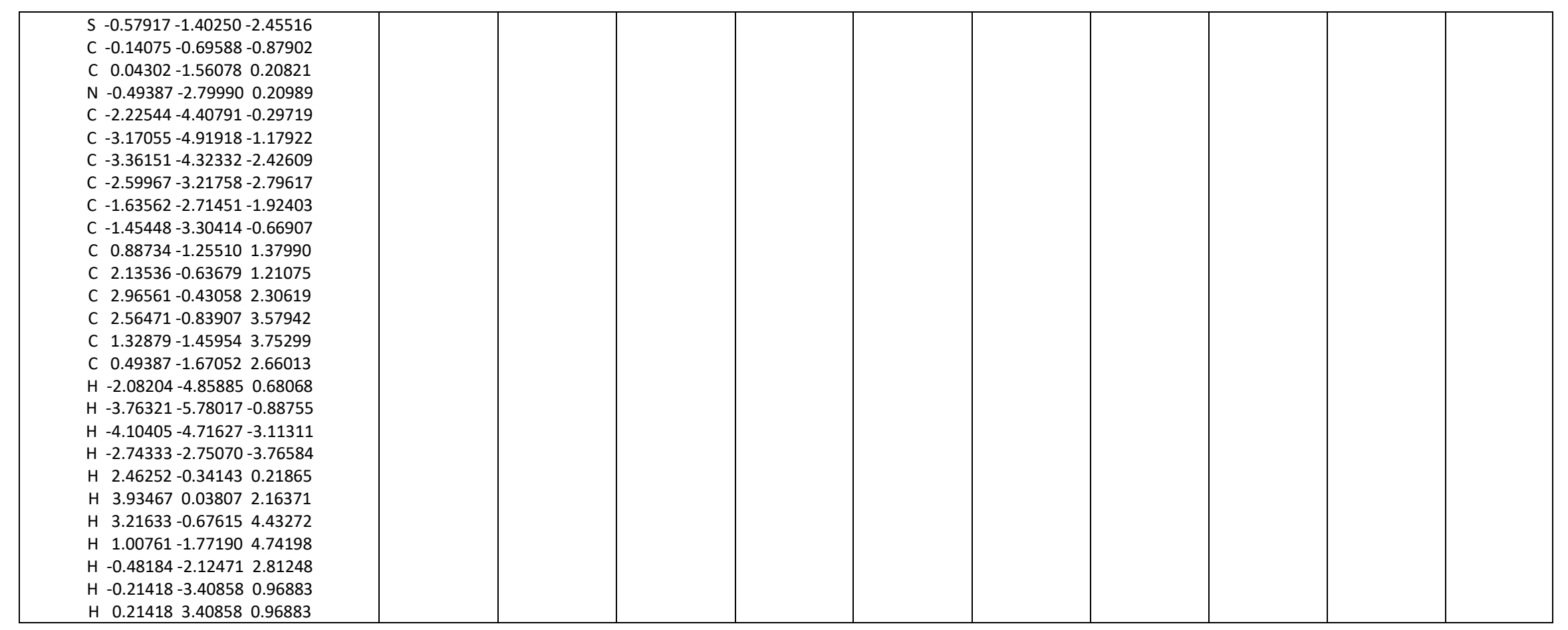




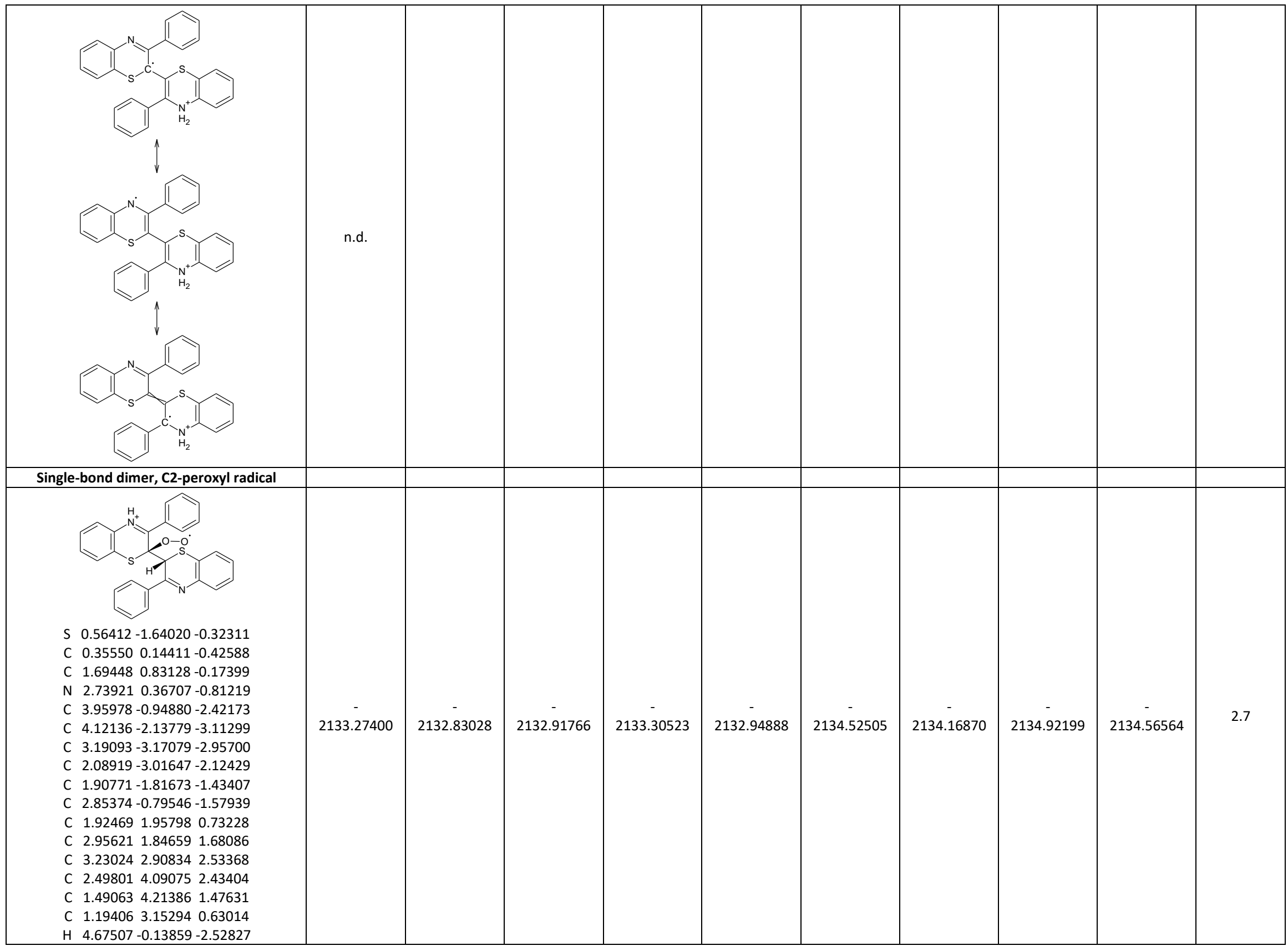




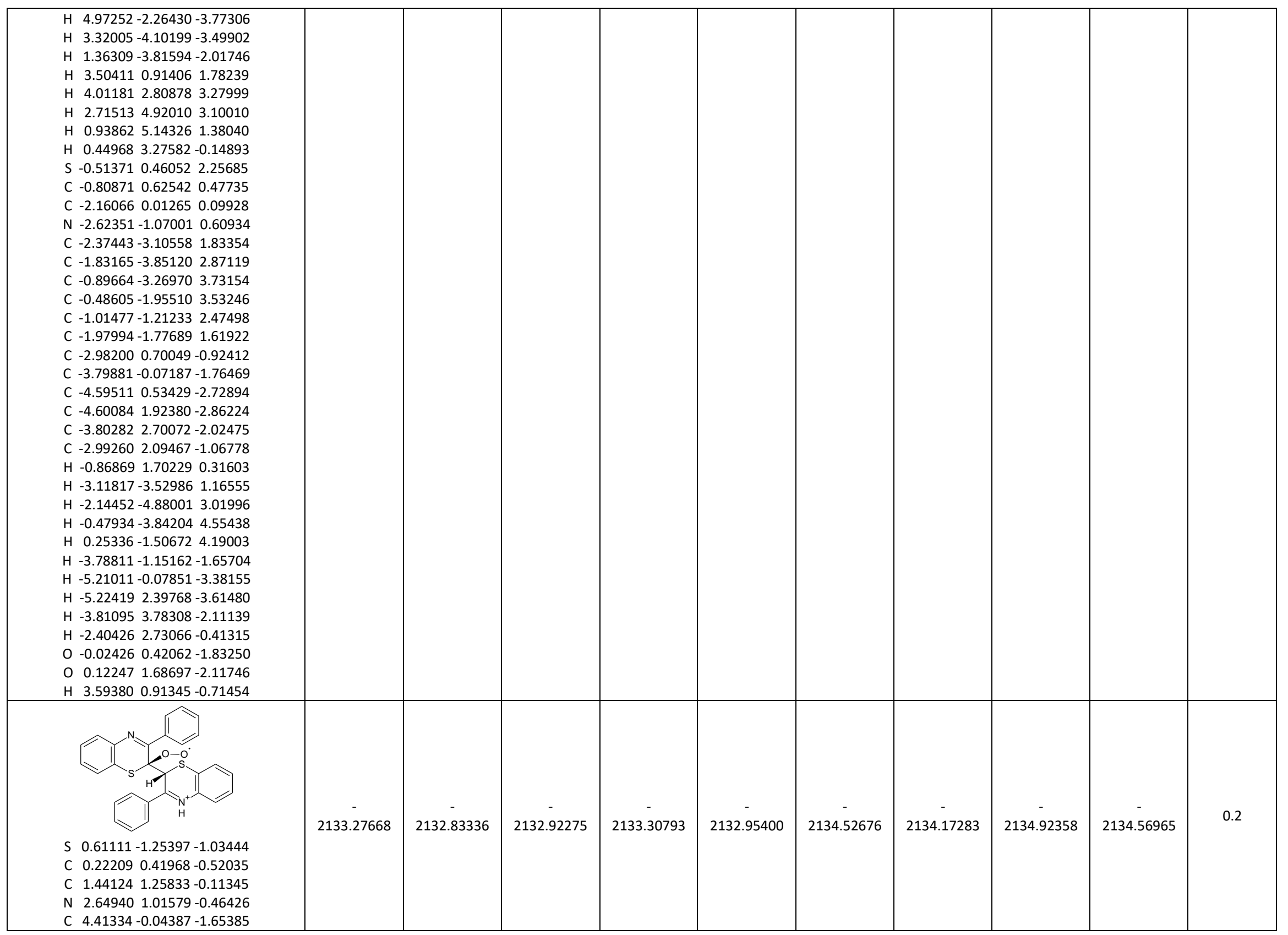


C $4.92802-1.08146-2.41715$ C $4.10873-2.15917-2.76456$ C $2.77909-2.19276-2.35679$ C $2.26577-1.14286-1.59337$ C $3.07600-0.05834-1.22848$ C $1.22325 \quad 2.489670 .69617$ C 2.100962 .750221 .75811 C 1.971843 .910322 .51306 C 0.970554 .834802 .21172 C $0.10325 \quad 4.59213 \quad 1.14875$ C 0.224123 .425770 .39509 H $5.026050 .80571-1.36776$ H $5.96354-1.05610-2.74116$ H $4.50335-2.97634-3.36056$ H $2.14228-3.02824-2.63364$ H $2.87762 \quad 2.02747 \quad 1.98797$ H 2.652464 .092973 .33948 H 0.870525 .741932 .80043 H $-0.66603 \quad 5.315250 .89472$ H $-0.43512 \quad 3.27498-0.45442$

$\begin{array}{lllll}\text { S } & -0.26303 & 0.03206 & 2.25262\end{array}$ $\begin{array}{llllllllll}\text { C } & -0.88374 & 0.38260 & 0.58146\end{array}$ C $-2.06209-0.486150 .22218$ N -2.12794 -1.720260 .65021$ C $-1.34026-3.809711 .54942$ C $-0.49734-4.513132 .39882$ C $0.43895-3.82664 \quad 3.17384$ C $0.54277-2.440203 .09909$ C $-0.29879-1.72275 \quad 2.24985$ C $-1.23651-2.420011 .48112$ C $-3.127330 .00771-0.64207$ C $-3.72355-0.85827-1.57835$ C $-4.75155-0.40146-2.39015$ C $-5.20605 \quad 0.91257-2.26936$ C $-4.62183 \quad 1.77515-1.34188$ C $-3.577891 .33503-0.53946$ H -1.25407 1.407780 .65694 H -2.07029 -4.328720 .93486$ H $-0.56991-5.593842 .45429$ H $1.09970-4.373833 .83849$ H $1.27643-1.911783 .69977$ H -3.34714 -1.86833 -1.71622 H -5.18952 -1.06695-3.12680 H -6.01268 1.26637-2.90392 H - $-4.98058 \quad 2.79422-1.24168$ H -3.15564 2.013090 .19495 O $-0.461651 .16653-1.62608$

O $0.225191 .15439-2.72922$ H -2.95716-2.24436 0.37773

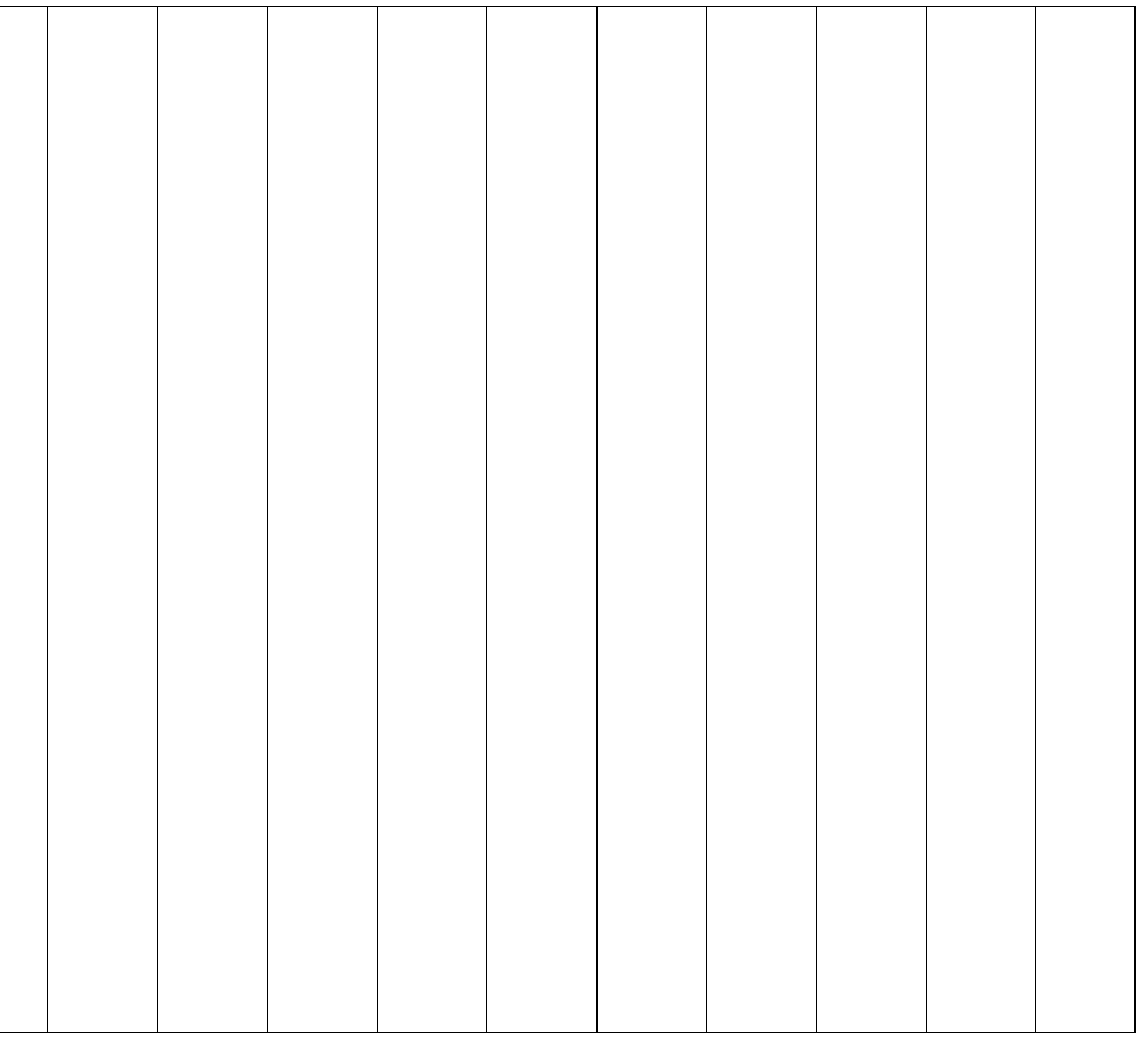




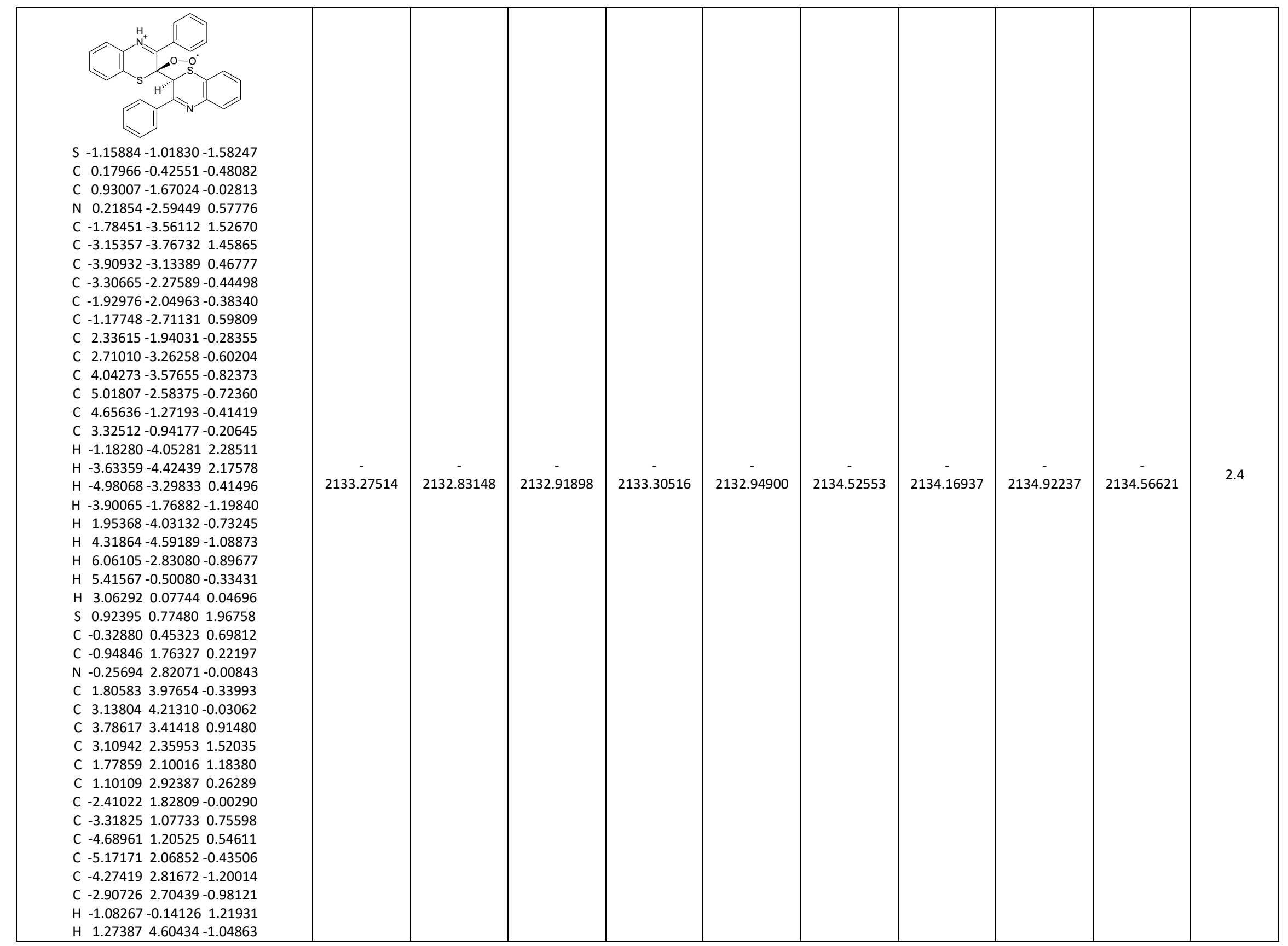




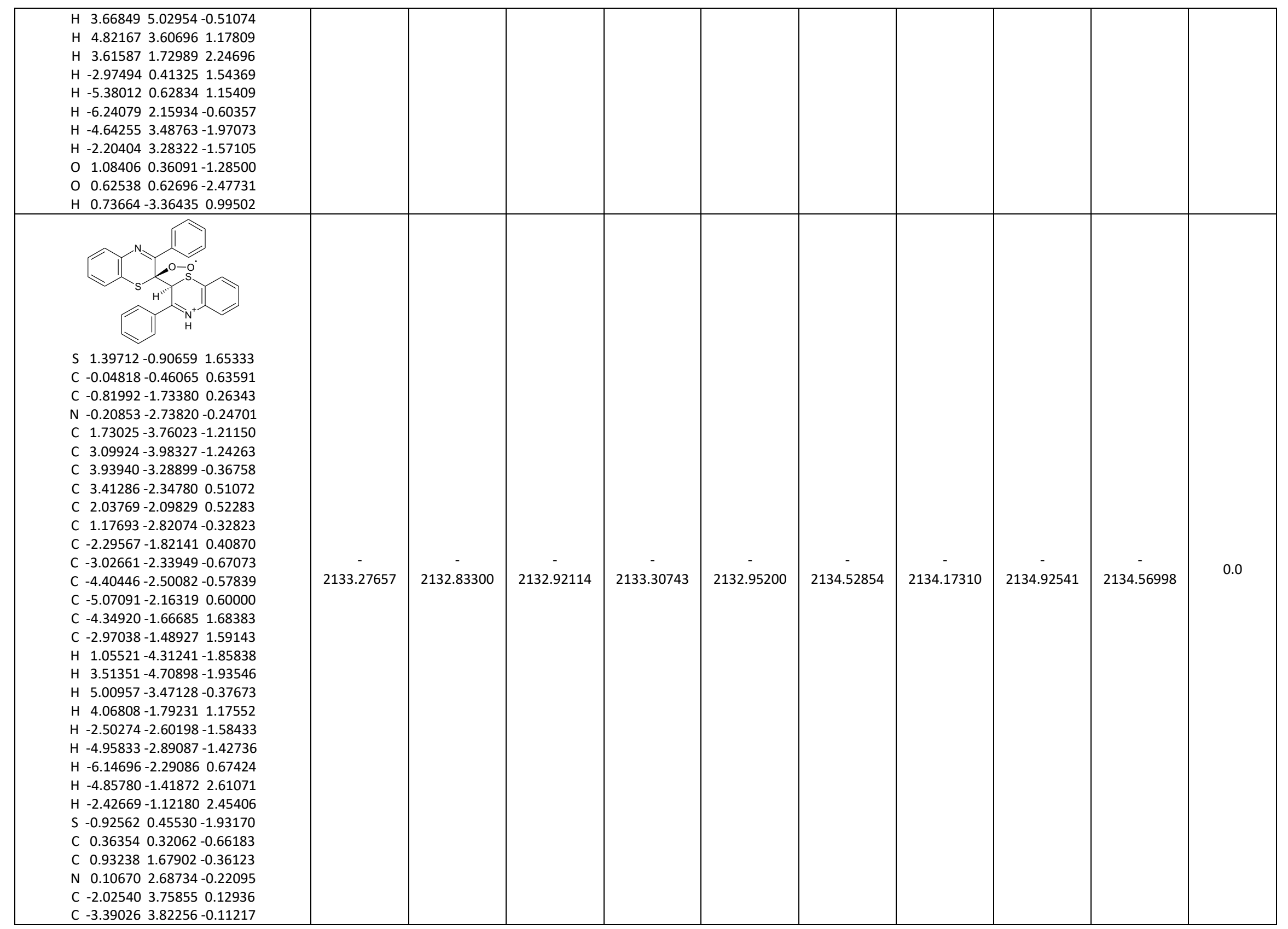




\begin{tabular}{|c|c|c|c|c|c|c|c|c|c|c|}
\hline $\begin{array}{lrrrr}\text { C } & -4.00882 & 2.84284 & -0.89234 \\
\text { C } & -3.27073 & 1.79078 & -1.42533 \\
\text { C } & -1.89872 & 1.71172 & -1.18581 \\
\text { C } & -1.28478 & 2.70525 & -0.41028 \\
\text { C } & 2.36033 & 1.89766 & -0.18028 \\
\text { C } & 3.29234 & 1.14666 & -0.91765 \\
\text { C } & 4.65161 & 1.39205 & -0.77402 \\
\text { C } & 5.09790 & 2.36434 & 0.11997 \\
\text { C } & 4.17992 & 3.10248 & 0.86991 \\
\text { C } & 2.81997 & 2.87974 & 0.71900 \\
\text { H } & 1.13780 & -0.28998 & -1.13339 \\
\text { H } & -1.53341 & 4.51192 & 0.73800 \\
\text { H } & -3.97201 & 4.63544 & 0.30884 \\
\text { H } & -5.07633 & 2.89339 & -1.08110 \\
\text { H } & -3.75576 & 1.02560 & -2.02310 \\
\text { H } & 2.96605 & 0.40545 & -1.63940 \\
\text { H } & 5.36302 & 0.82242 & -1.36291 \\
\text { H } & 6.16200 & 2.54409 & 0.23807 \\
\text { H } & 4.52623 & 3.84356 & 1.58279 \\
\text { H } & 2.12373 & 3.42720 & 1.34841 \\
\text { O } & -0.90095 & 0.40237 & 1.41973 \\
\text { O } & -0.27803 & 0.98992 & 2.40820 \\
\text { H } & 0.52358 & 3.59152 & -0.01263 \\
\end{array}$ & & & & & & & & & & \\
\hline $\begin{array}{llll}\text { S } & -2.10883 & -0.73723 & -1.35147 \\
C & -0.88997 & -0.30485 & -0.07852 \\
C & -1.11733 & 1.07876 & 0.52258 \\
N & -2.32938 & 1.57036 & 0.60579 \\
C & -4.71232 & 1.79757 & 0.43571 \\
C & -5.92513 & 1.39615 & -0.10068 \\
C & -5.96849 & 0.32984 & -1.00329 \\
C & -4.80543 & -0.34077 & -1.36444 \\
C & -3.57884 & 0.05607 & -0.82912 \\
C & -3.54103 & 1.12993 & 0.06327 \\
C & -0.04589 & 1.82292 & 1.18020 \\
C & 0.95711 & 1.15914 & 1.90708 \\
C & 1.91990 & 1.89644 & 2.58682 \\
C & 1.90672 & 3.28929 & 2.53620 \\
C & 0.92107 & 3.95402 & 1.80414 \\
C & -0.05326 & 3.22933 & 1.13312 \\
H & -4.66461 & 2.62159 & 1.14220 \\
H & -6.83610 & 1.91110 & 0.18424 \\
H & -6.91723 & 0.01281 & -1.42422\end{array}$ & $\begin{array}{c}- \\
- \\
2133.27770\end{array}$ & 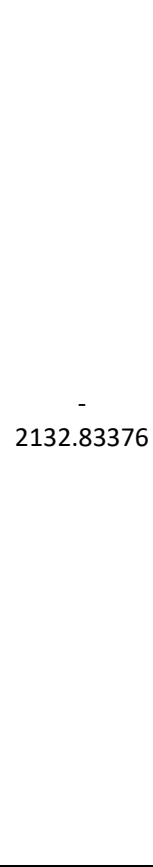 & 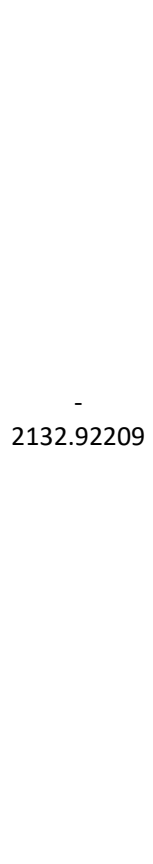 & 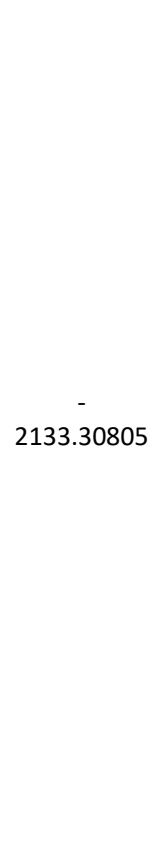 & 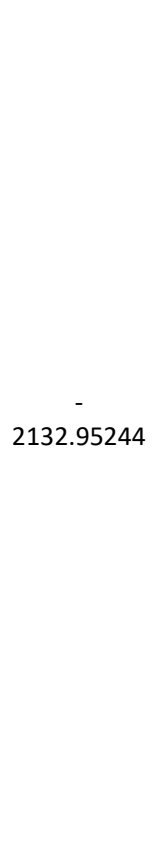 & 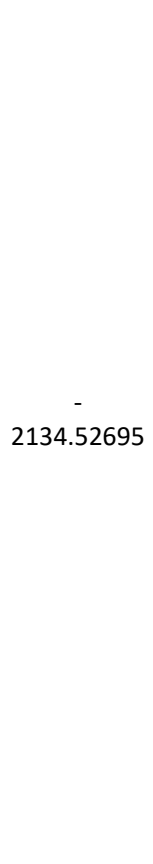 & 要 & $\begin{array}{c} \\
- \\
2134.92435\end{array}$ & $\begin{array}{l}- \\
- \\
- \\
2134.56875\end{array}$ & 0.8 \\
\hline
\end{tabular}




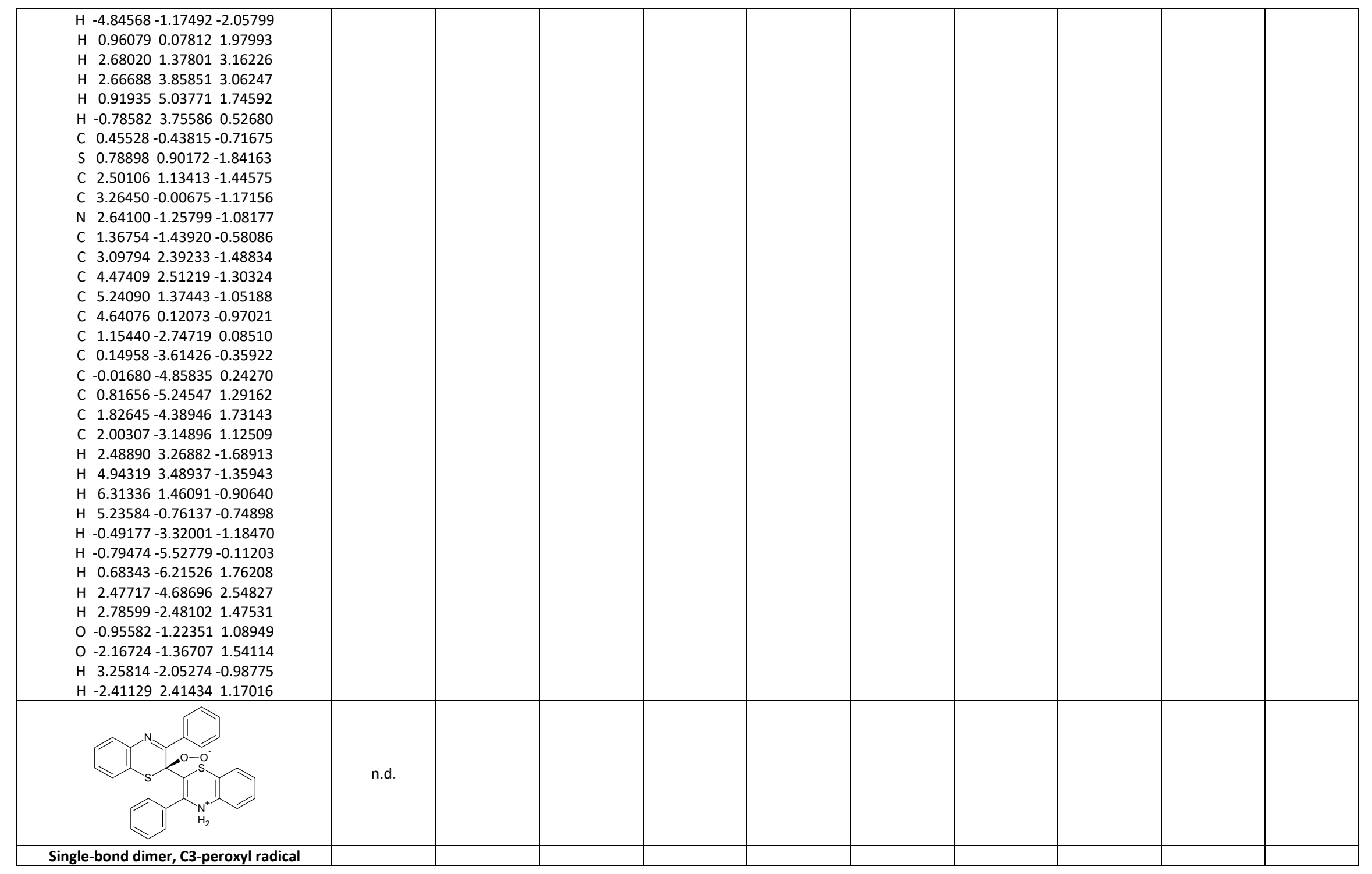




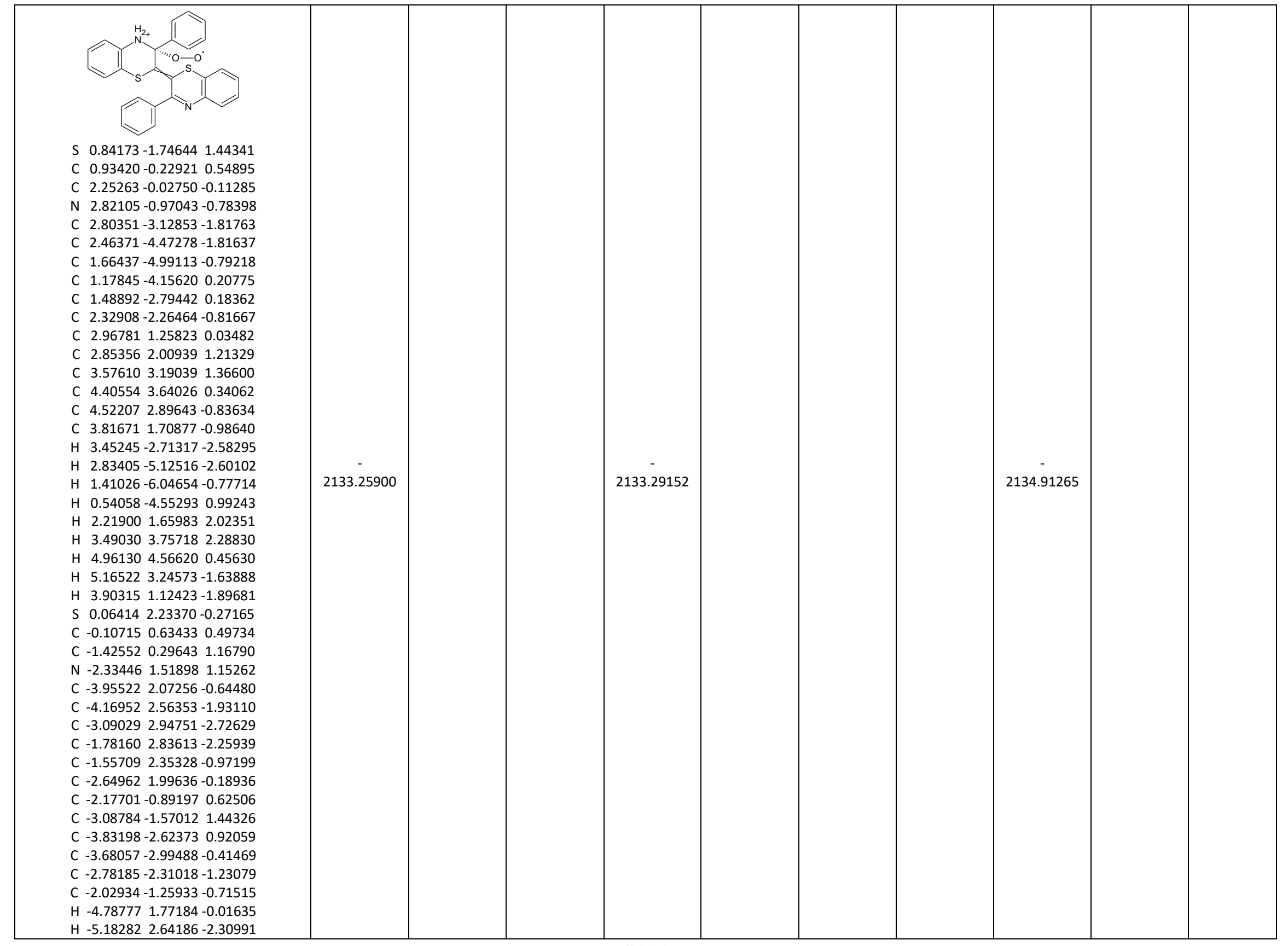




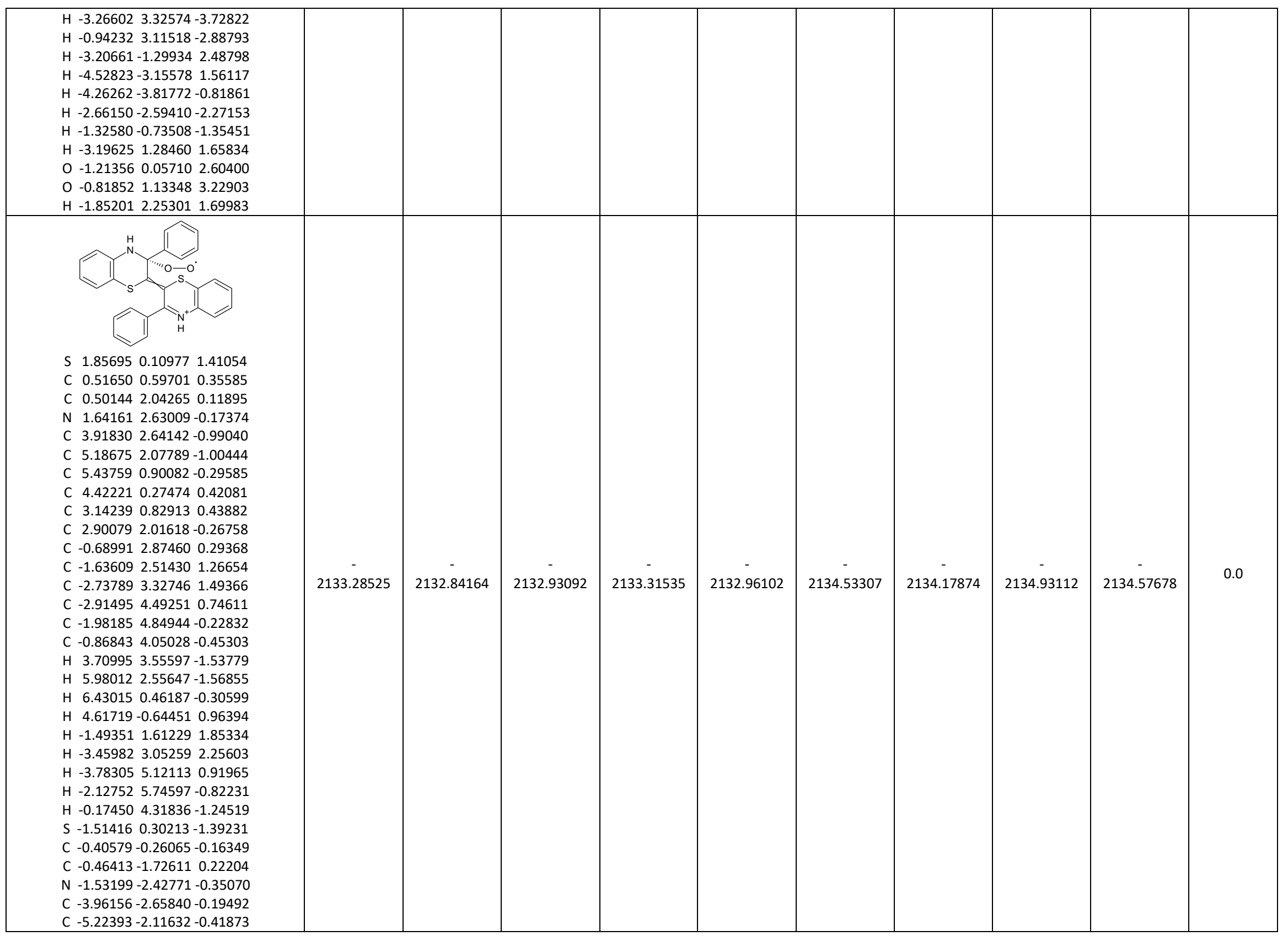




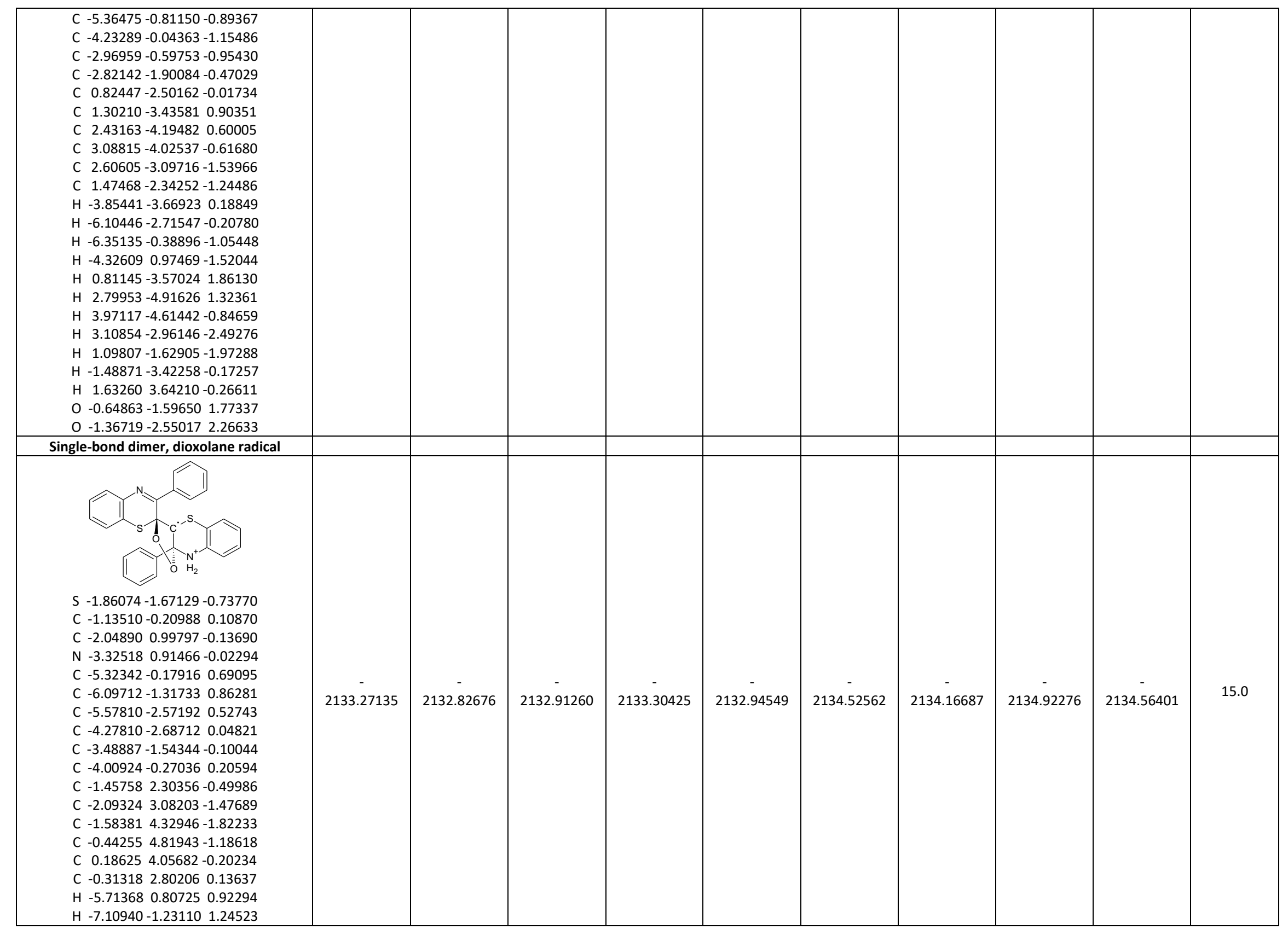




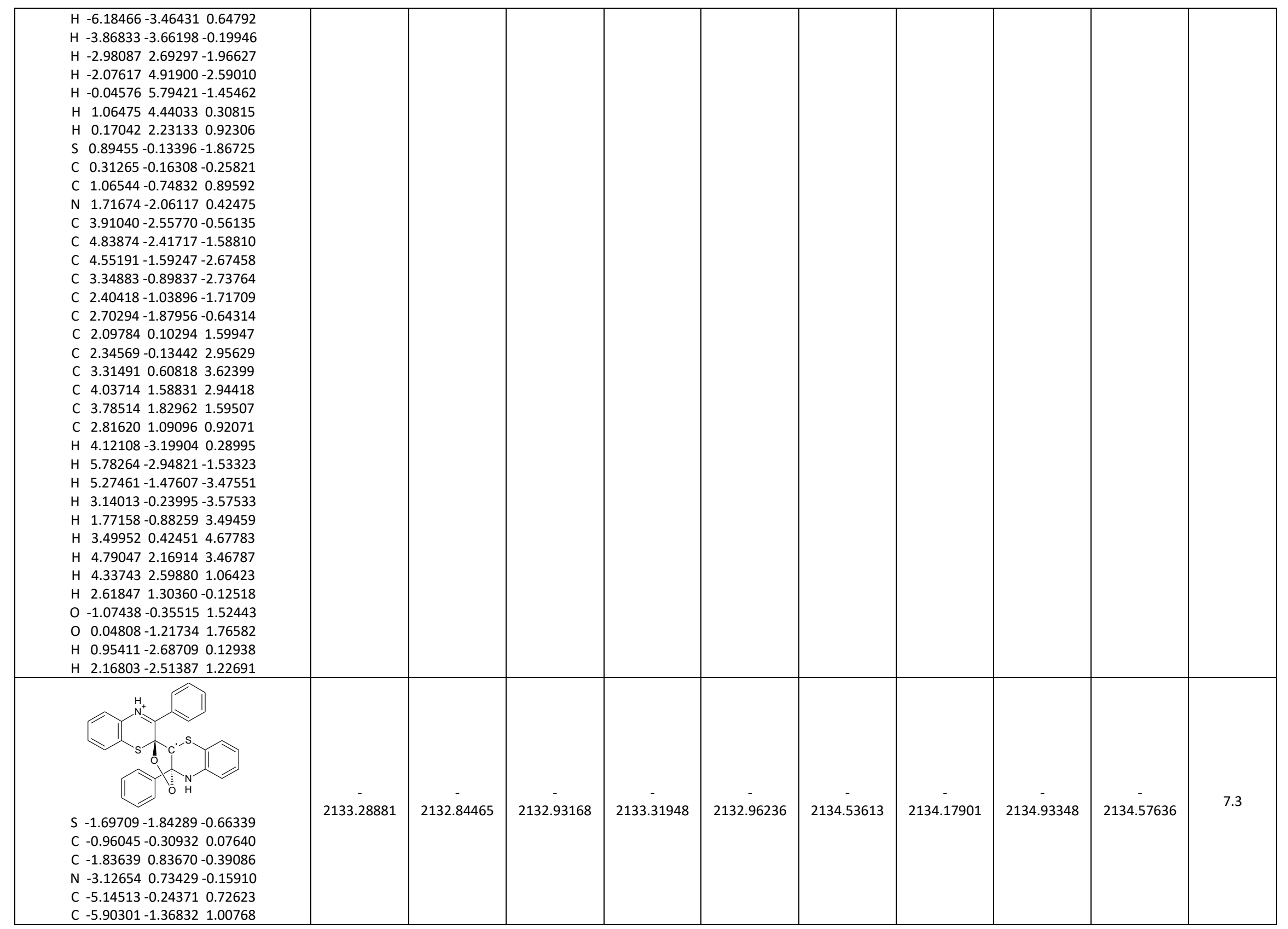

S114 
C $-5.37450-2.643710 .78035$ C $-4.08525-2.800040 .28754$ C $-3.30401-1.675180 .00634$ C -3.85044 $-0.40053 \quad 0.22086$ C - $1.34508 \quad 2.03200-1.05475$ C $-2.107992 .59916-2.09449$ C - $1.678353 .76441-2.71359$ C $-0.497154 .38048-2.29754$ C $0.260623 .82582-1.26531$ C $-0.149862 .65165-0.64902$ H -5.543790 .752650 .89383$ H -6.90665 - 1.255021 .40310 H -5.96979-3.52393 1.00104 H -3.67426-3.79167 0.12750 H $-3.004752 .10066-2.45210$ H - 2.25780 $4.18539-3.52870$ H -0.16365 $5.29262-2.78299$ H $1.173594 .31140-0.93599$ H $0.43260 \quad 2.24022 \quad 0.16748$ S $1.16265-0.57083-1.75296$ C $0.49987-0.30711-0.20843$ C $1.19076-0.59721 \quad 1.10286$ N 2.11533-1.69043 1.01880 C $4.15446-2.592250 .11075$ C $5.01451-2.83952-0.94928$ C $4.71256-2.37187-2.23062$ C $3.53679-1.66294-2.43174$ C 2.65637-1.43126-1.36906 C $2.95603-1.88511-0.07494$ C 1.793110 .645921 .75350 C 1.319071 .135112 .97228 C 1.906382 .261073 .54978 C 2.965362 .905912 .91491 C 3.440672 .419611 .69600 C $2.85778 \quad 1.296671 .11659$ H $4.40137-2.948601 .10756$ H $5.93178-3.39222-0.77055$ H $5.38583-2.55361-3.06172$ H $3.28711-1.28676-3.42038$ H 0.494790 .636313 .47034 H 1.531652 .632834 .49908 H 3.419853 .783263 .36577 H 4.266712 .915381 .19451 H 3.237860 .930540 .16633 O $-1.02292-0.296411 .49016$ O $0.08849-1.102061 .89418$ H $2.58175-1.858651 .90264$ H -3.67966 $1.57087-0.33254$ 


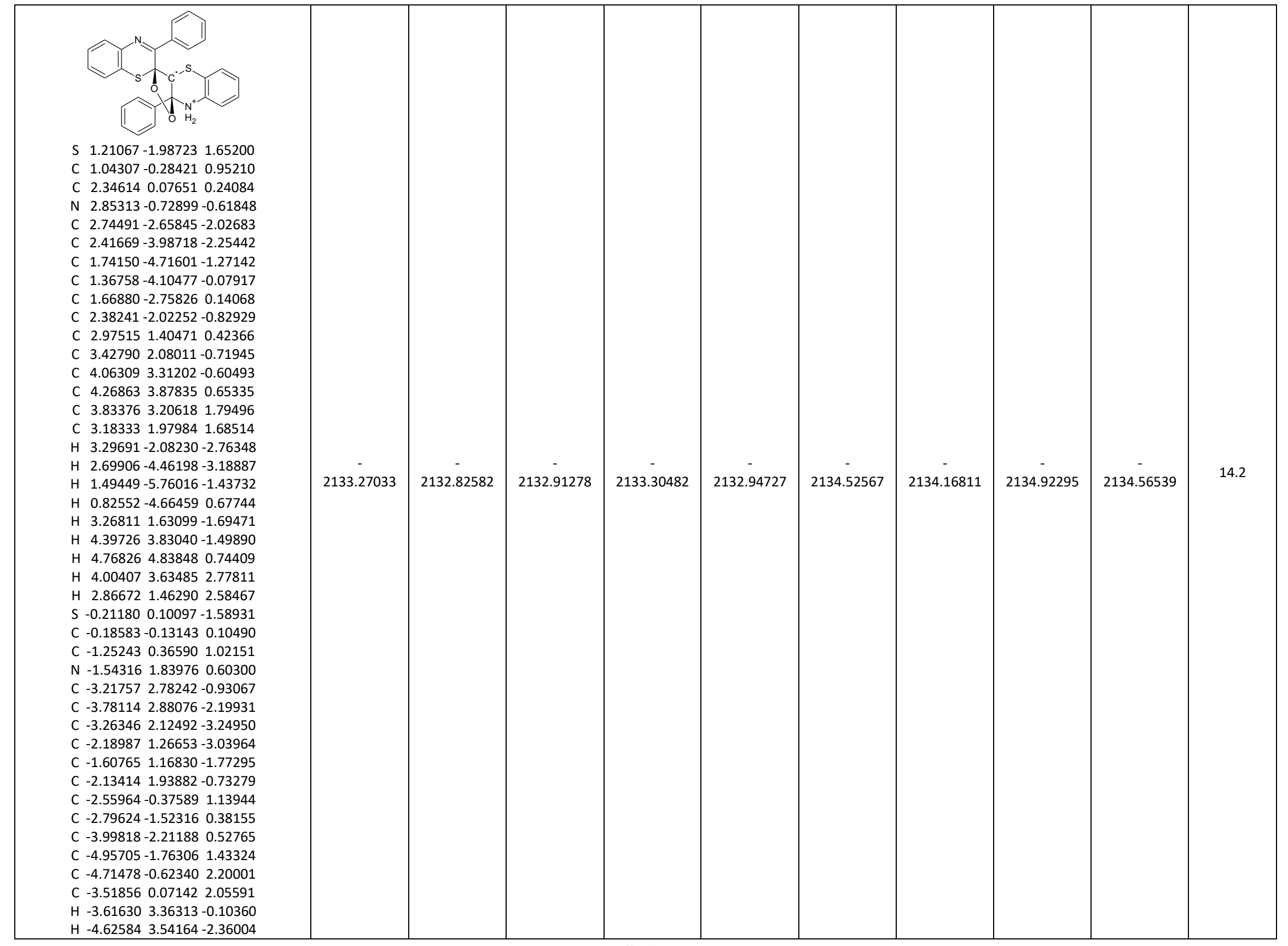




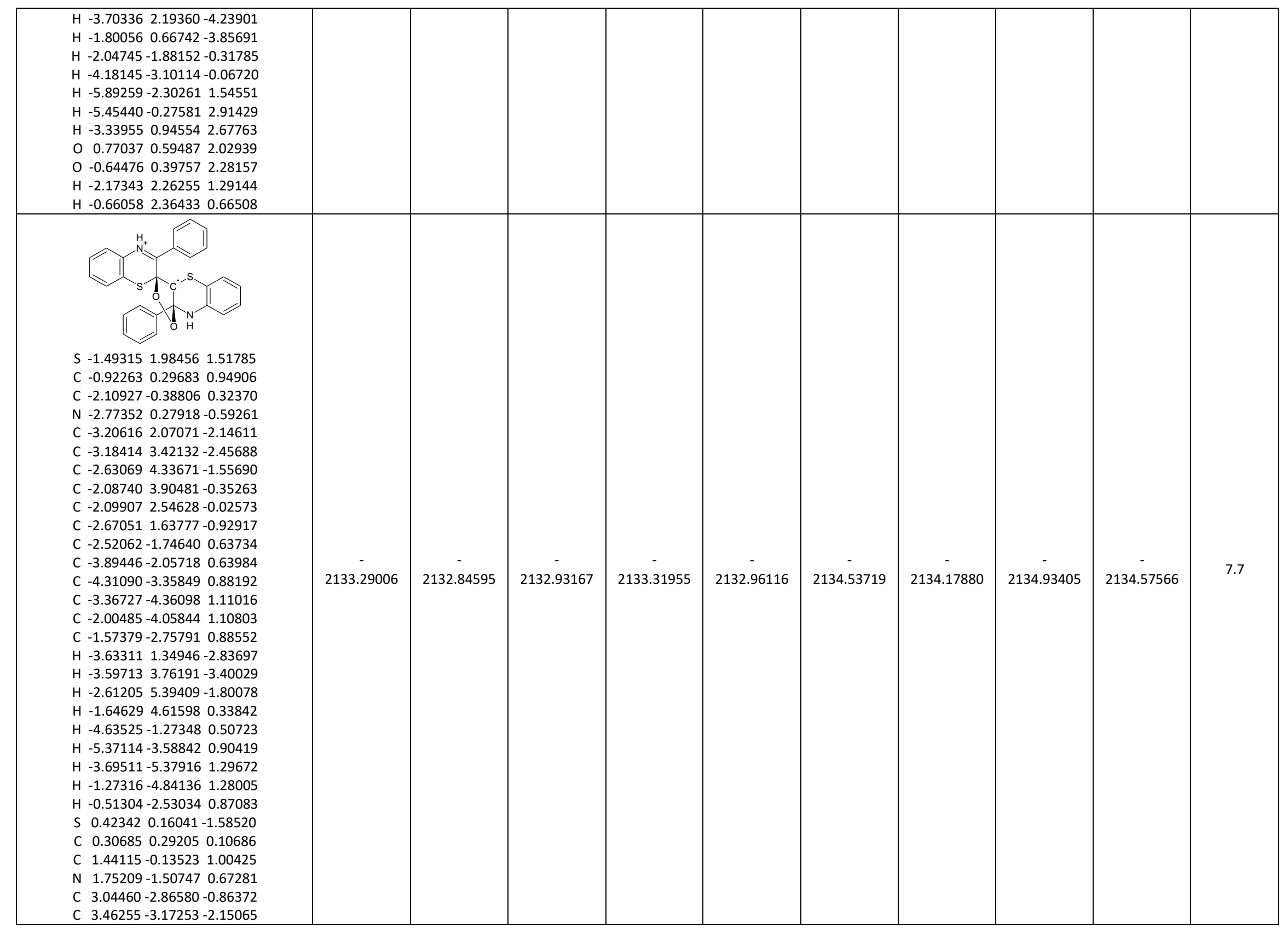




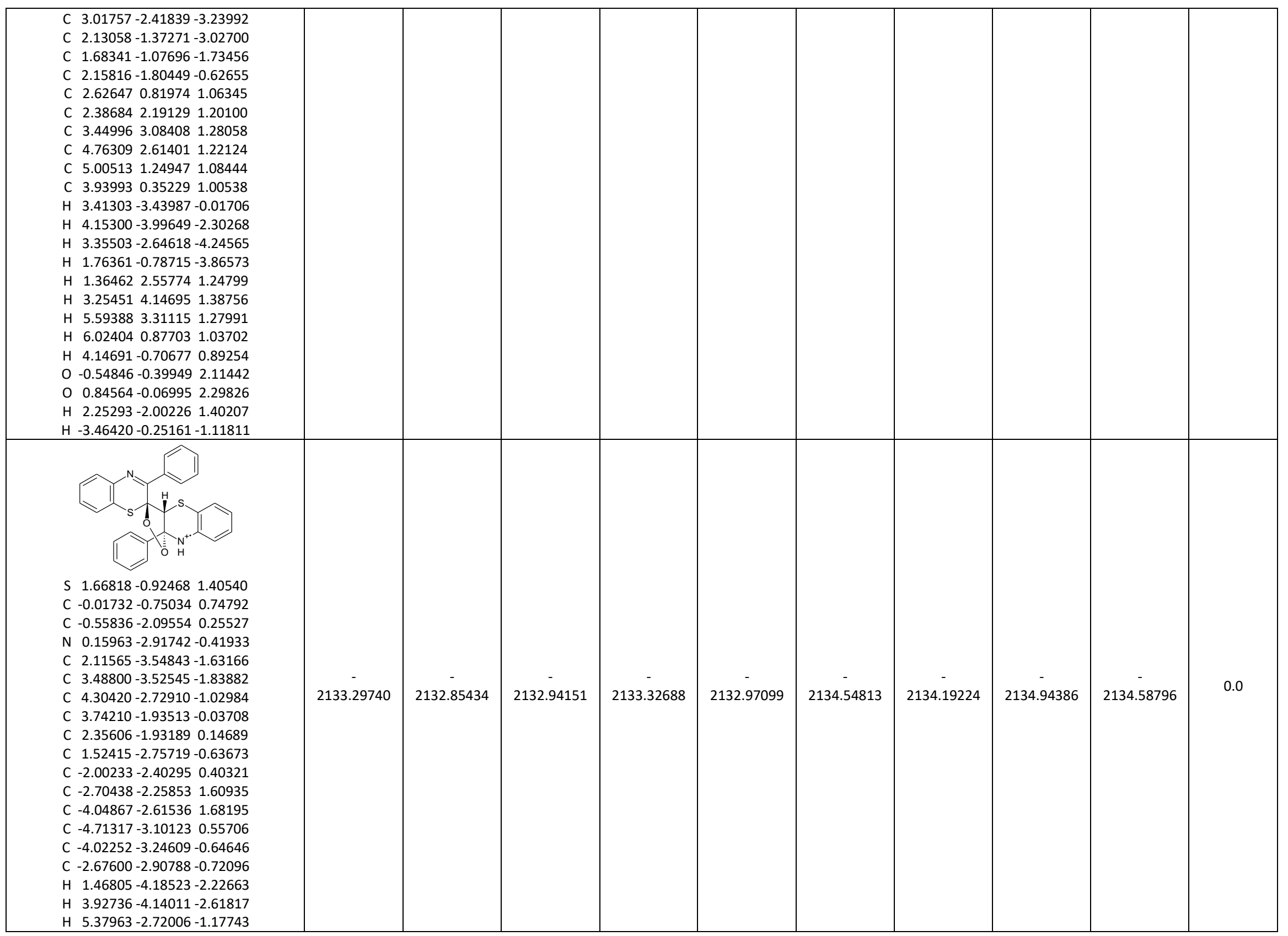




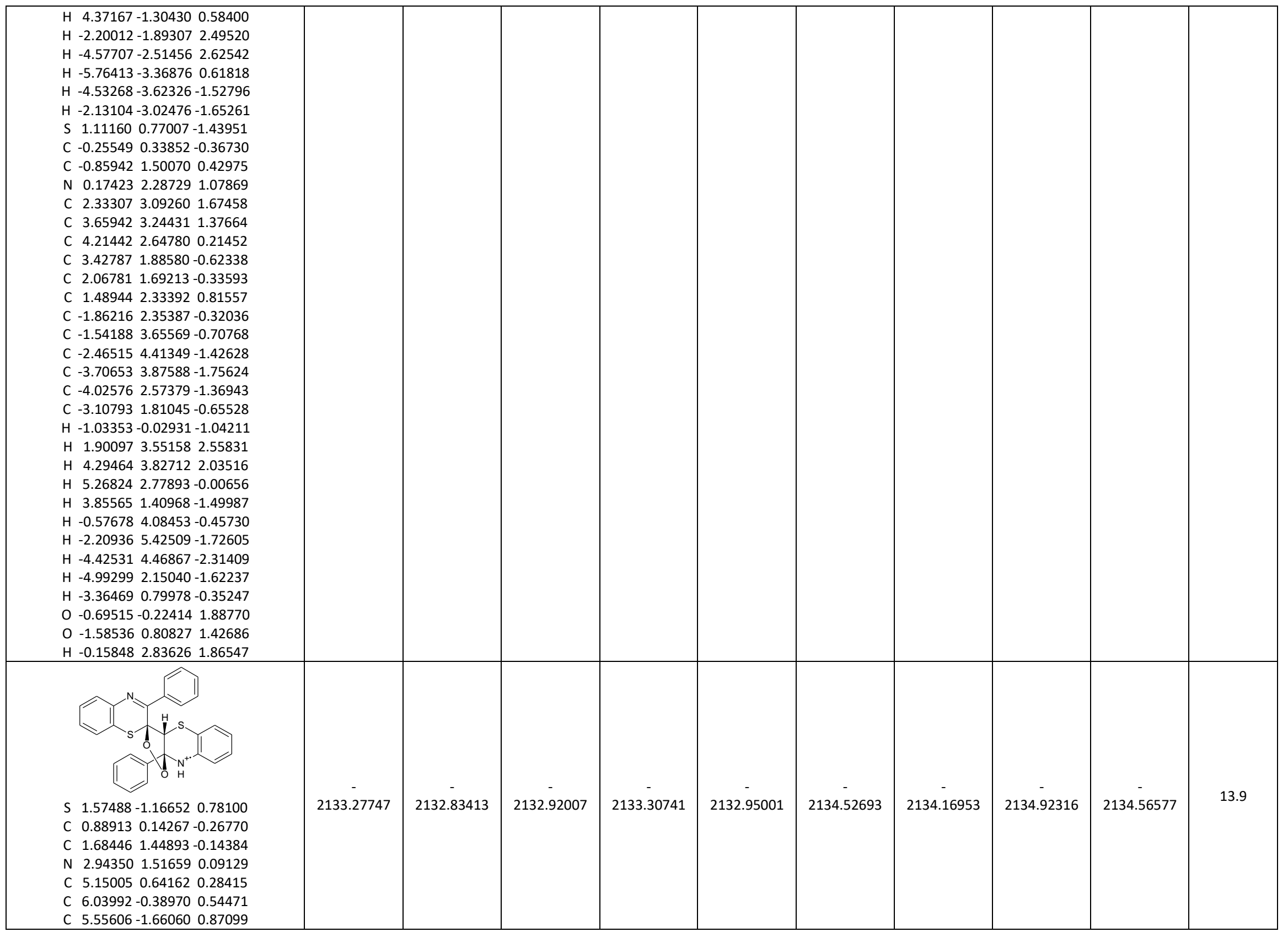


C $4.18735-1.899180 .91899$

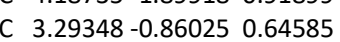

C 3.763950 .424320 .33212

C $0.978632 .73946-0.37188$

C $0.224182 .97996-1.52931$

C $-0.378434 .22061-1.72610$

C $-0.244305 .22667-0.76995$

C 0.506564 .993060 .38186

C 1.120033 .758770 .57775

H 5.498931 .639930 .03736

H $7.10914-0.207860 .50095$

H $6.24708-2.470911 .08231$

H $3.80981-2.888561 .16142$

$0.132992 .21197-2.29221$

H $-0.947864 .40180-2.63297$

H $-0.719416 .19102-0.92452$

H 0.616895 .773451 .12911

H 1.711203 .573831 .46966

S -1.271700 .164701 .65035$

C $-0.607290 .24581-0.01176$

C $-1.18990-0.77713-0.99269$

N $-2.57858-0.40295-1.18132$

C $-4.764290 .23298-0.51864$

C -5.649900 .611880 .44961$

$\begin{array}{llll}C & -5.20702 & 0.83543 & 1.77782\end{array}$

$\begin{array}{llll}-3.87274 & 0.69378 & 2.10984\end{array}$

C -2.928130 .328151 .14335$

C $-3.383750 .05766-0.20669$

C $-1.07794-2.27662-0.75348$

C $-0.24972-3.04274-1.58013$

C $-0.14823-4.41770-1.38840$

C $-0.87261-5.04100-0.37447$

C $-1.71416-4.284040 .43946$

C - $-1.82449-2.910140 .24691$

H $-0.924561 .21582-0.40764$

H -5.09259 $0.04979-1.53701$

H -6.698080 .739540 .20244$

$\begin{array}{llll}H & -5.91862 & 1.13685 & 2.53897\end{array}$

H -3.539930 .888593 .12430$

H $0.32083-2.56330-2.36687$

H $0.50027-5.00094-2.03511$

H $-0.79155-6.11362-0.22533$

$H-2.29524-4.762191 .22195$

H $-2.50484-2.351890 .88077$

$0.89805-0.20259-1.68428$

O $-0.47126-0.37890-2.15151$

H - $-3.01967-0.71367-2.04291$

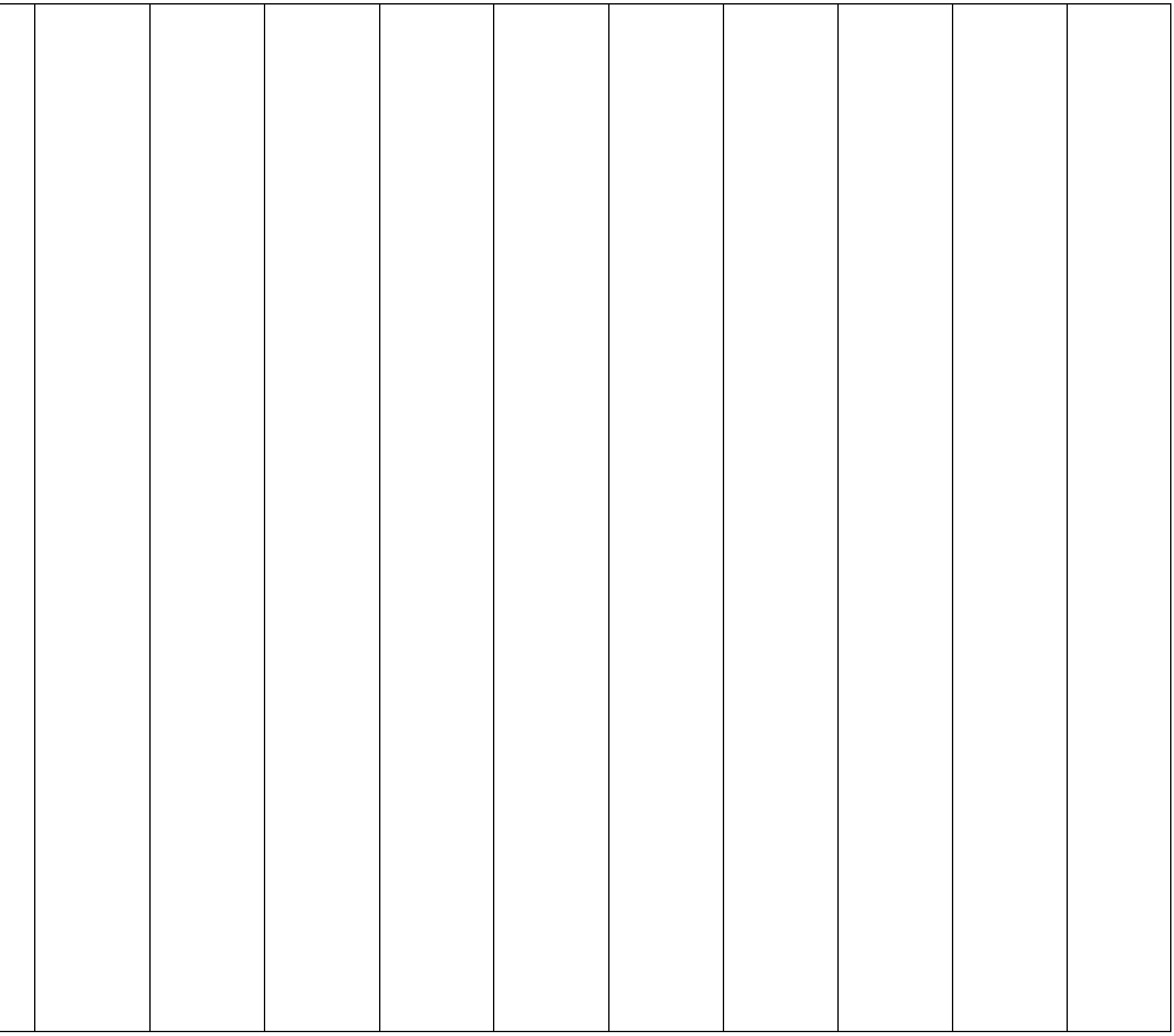




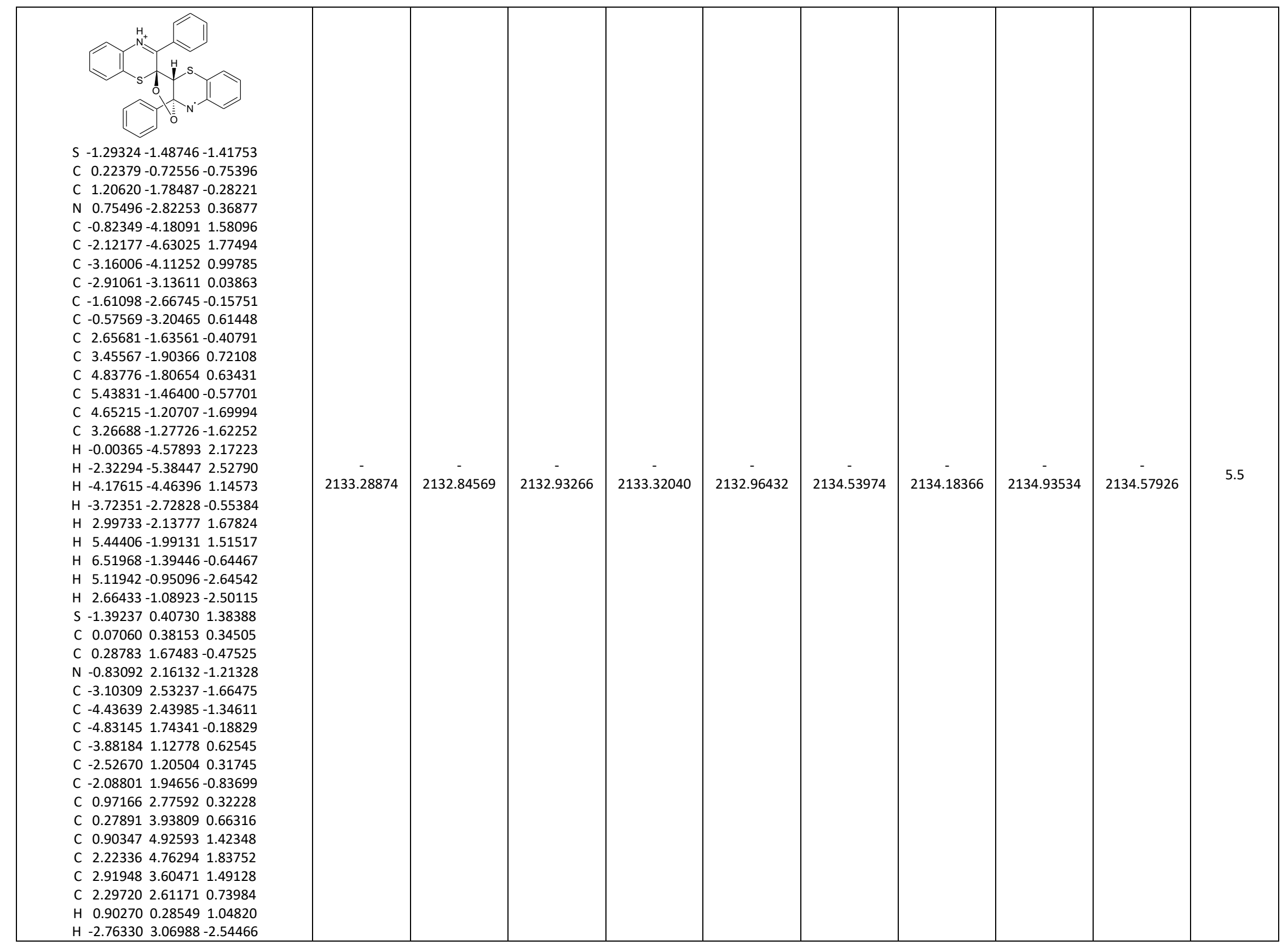




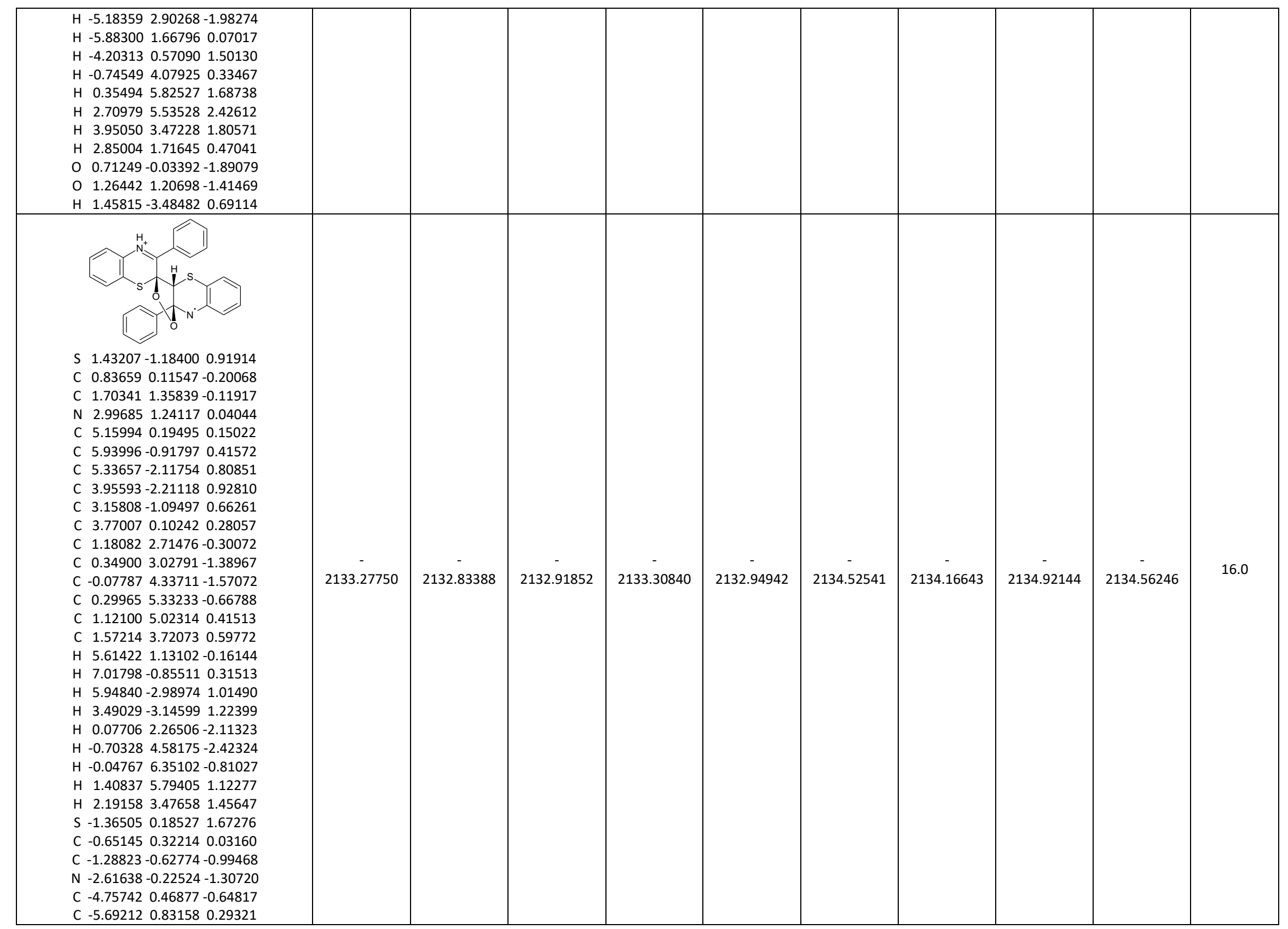




\begin{tabular}{|c|c|c|c|c|c|c|c|c|c|c|}
\hline $\begin{array}{lllll}\text { C } & -5.31095 & 0.96158 & 1.63929 \\
\text { C } & -3.98649 & 0.75234 & 2.02026 \\
\text { C } & -3.02047 & 0.38904 & 1.08637 \\
\text { C } & -3.39535 & 0.19889 & -0.29935 \\
\text { C } & -1.24524 & -2.14149 & -0.76946 \\
\text { C } & -0.45670 & -2.94458 & -1.60063 \\
\text { C } & -0.43292 & -4.32699 & -1.43377 \\
\text { C } & -1.19954 & -4.92859 & -0.43794 \\
\text { C } & -2.00594 & -4.13739 & 0.37781 \\
\text { C } & -2.03790 & -2.75520 & 0.20745 \\
\text { H } & -0.90214 & 1.32335 & -0.32791 \\
\text { H } & -5.02284 & 0.34747 & -1.69379 \\
\text { H } & -6.72021 & 1.01479 & -0.00190 \\
\text { H } & -6.04165 & 1.24576 & 2.38981 \\
\text { H } & -3.69841 & 0.89096 & 3.05858 \\
\text { H } & 0.14542 & -2.48952 & -2.37799 \\
\text { H } & 0.18804 & -4.93304 & -2.08713 \\
\text { H } & -1.17964 & -6.00663 & -0.30689 \\
\text { H } & -2.62416 & -4.59440 & 1.14489 \\
\text { H } & -2.69834 & -2.17049 & 0.83713 \\
\text { O } & 0.88204 & -0.27883 & -1.59039 \\
\text { O } & -0.46347 & -0.26663 & -2.12268 \\
\text { H } & 3.52524 & 2.10759 & -0.04878 \\
\end{array}$ & & & & & & & & & & \\
\hline $\begin{array}{lllll} & & & & \\
\text { S } & 0.85855 & 2.29919 & -1.63638 \\
\mathrm{C} & -0.01408 & 0.82983 & -0.96715 \\
\mathrm{C} & -1.39243 & 1.28605 & -0.49097 \\
\mathrm{~N} & -1.49659 & 2.28338 & 0.31023 \\
\mathrm{C} & -0.52271 & 3.86502 & 1.82185 \\
\mathrm{C} & 0.45994 & 4.78682 & 2.15290 \\
\mathrm{C} & 1.55594 & 4.97472 & 1.30560 \\
\mathrm{C} & 1.68350 & 4.21556 & 0.14742 \\
\mathrm{C} & 0.71352 & 3.26165 & -0.17127 \\
\mathrm{C} & -0.41812 & 3.09042 & 0.65583 \\
\mathrm{C} & -2.62611 & 0.55295 & -0.86212 \\
\mathrm{C} & -2.88725 & 0.11228 & -2.16775 \\
\mathrm{C} & -4.09339 & -0.51596 & -2.46615 \\
\mathrm{C} & -5.04491 & -0.72648 & -1.46903 \\
\mathrm{C} & -4.79260 & -0.29001 & -0.16827 \\
\mathrm{C} & -3.59676 & 0.35316 & 0.13152 \\
\mathrm{H} & -1.39773 & 3.72673 & 2.44986 \\
\mathrm{H} & 0.36738 & 5.37238 & 3.06230 \\
\mathrm{H} & 2.31954 & 5.70572 & 1.55302 \\
\end{array}$ & 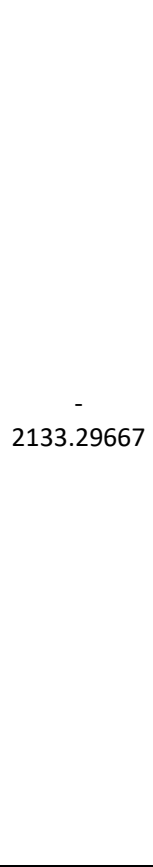 & 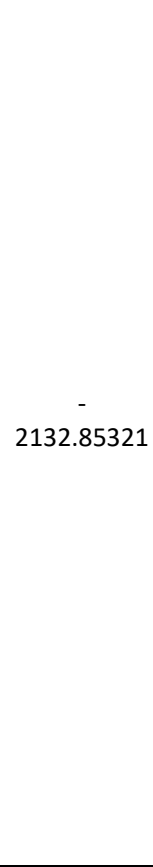 & 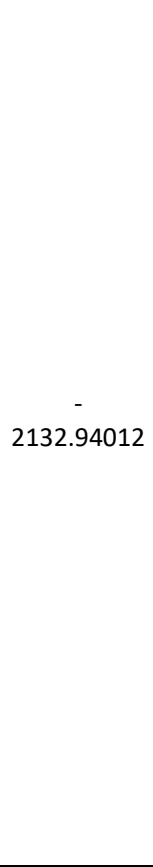 & 更 & $\begin{array}{c}-2 \\
- \\
2132.97021\end{array}$ & 更 & 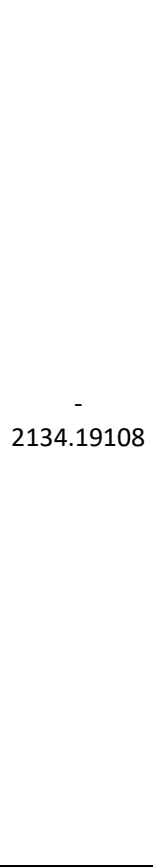 & $\begin{array}{c}- \\
- \\
2134.94367\end{array}$ & $\begin{array}{c}- \\
- \\
2134.58711\end{array}$ & 0.5 \\
\hline
\end{tabular}




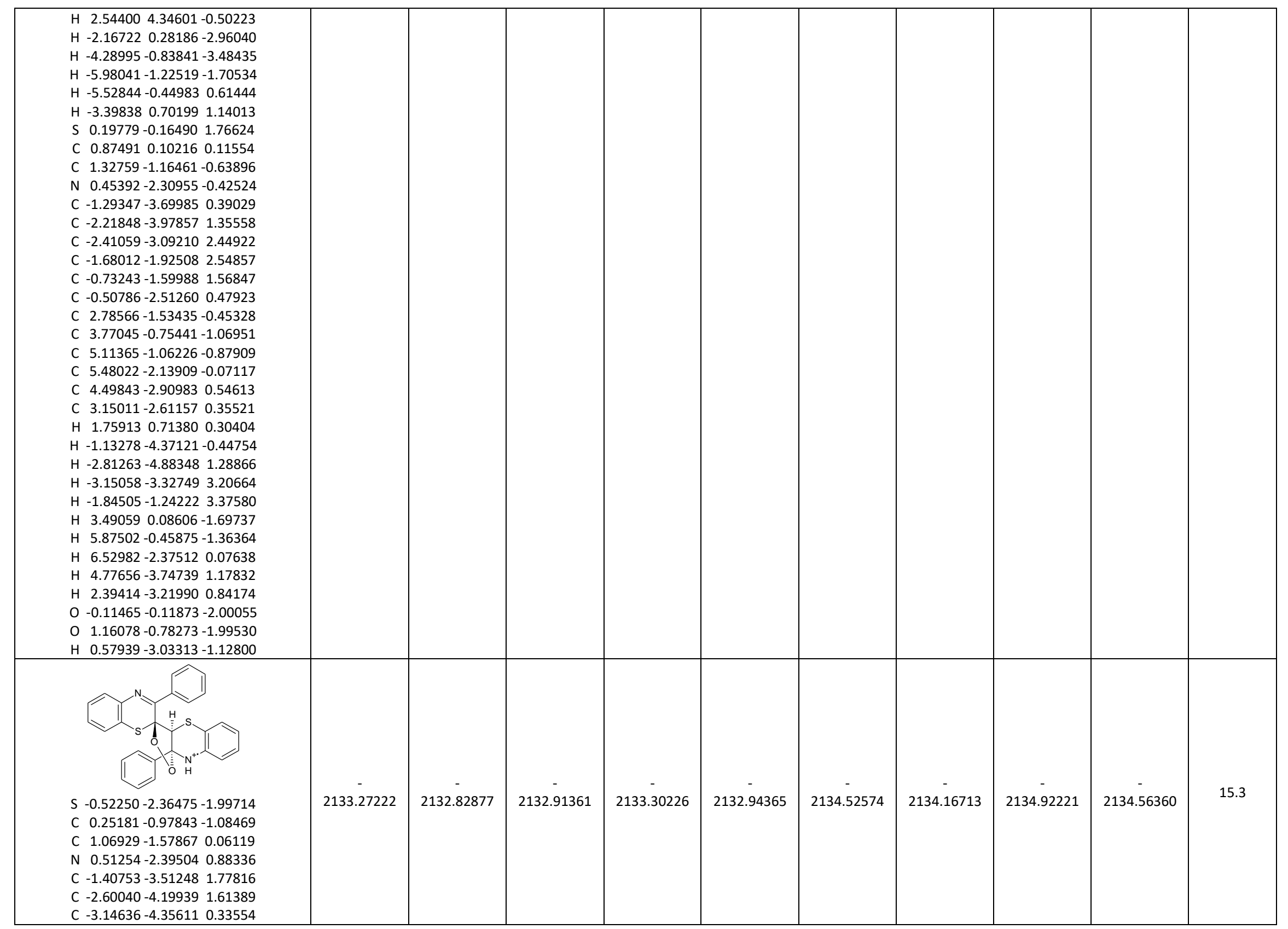




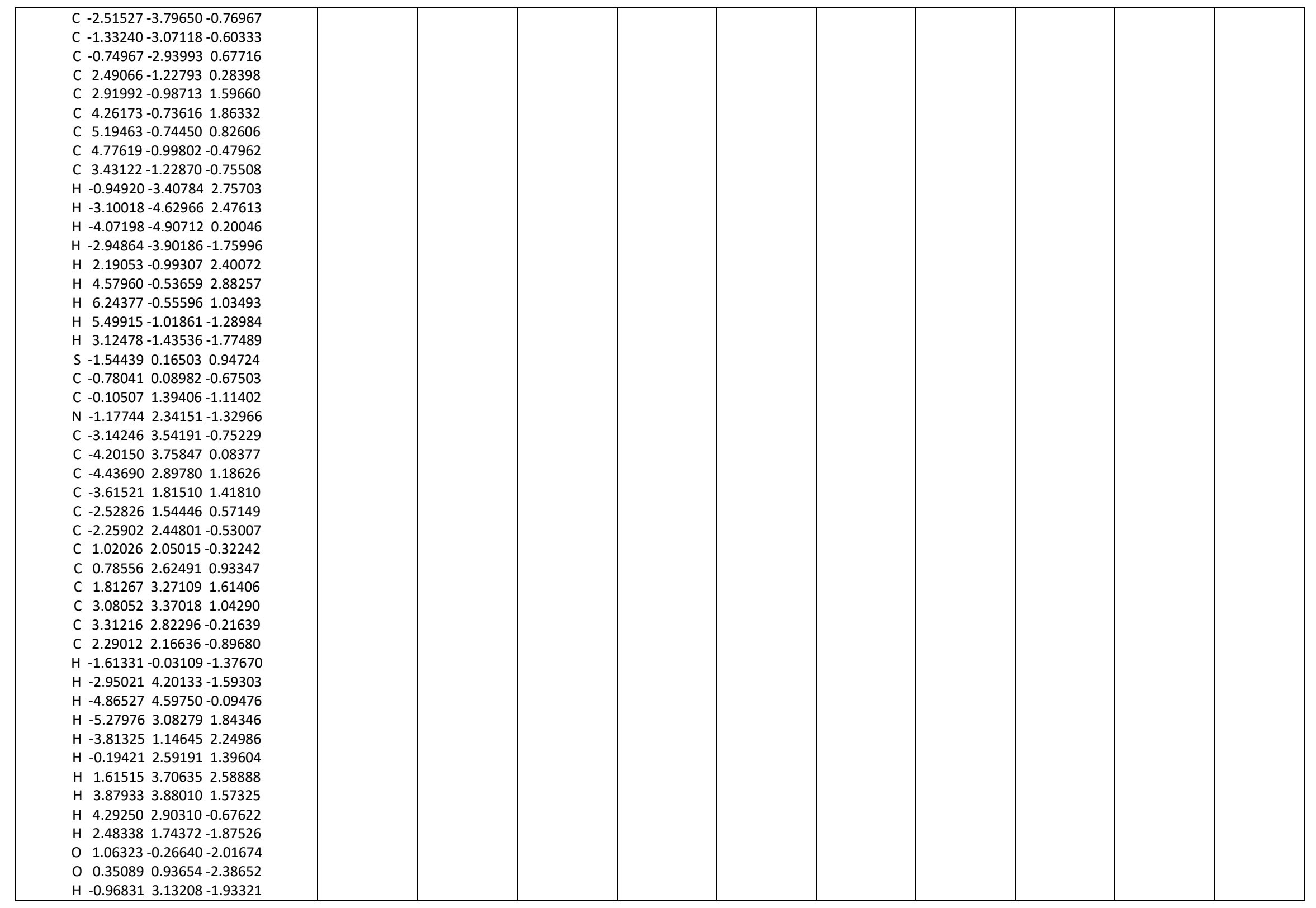




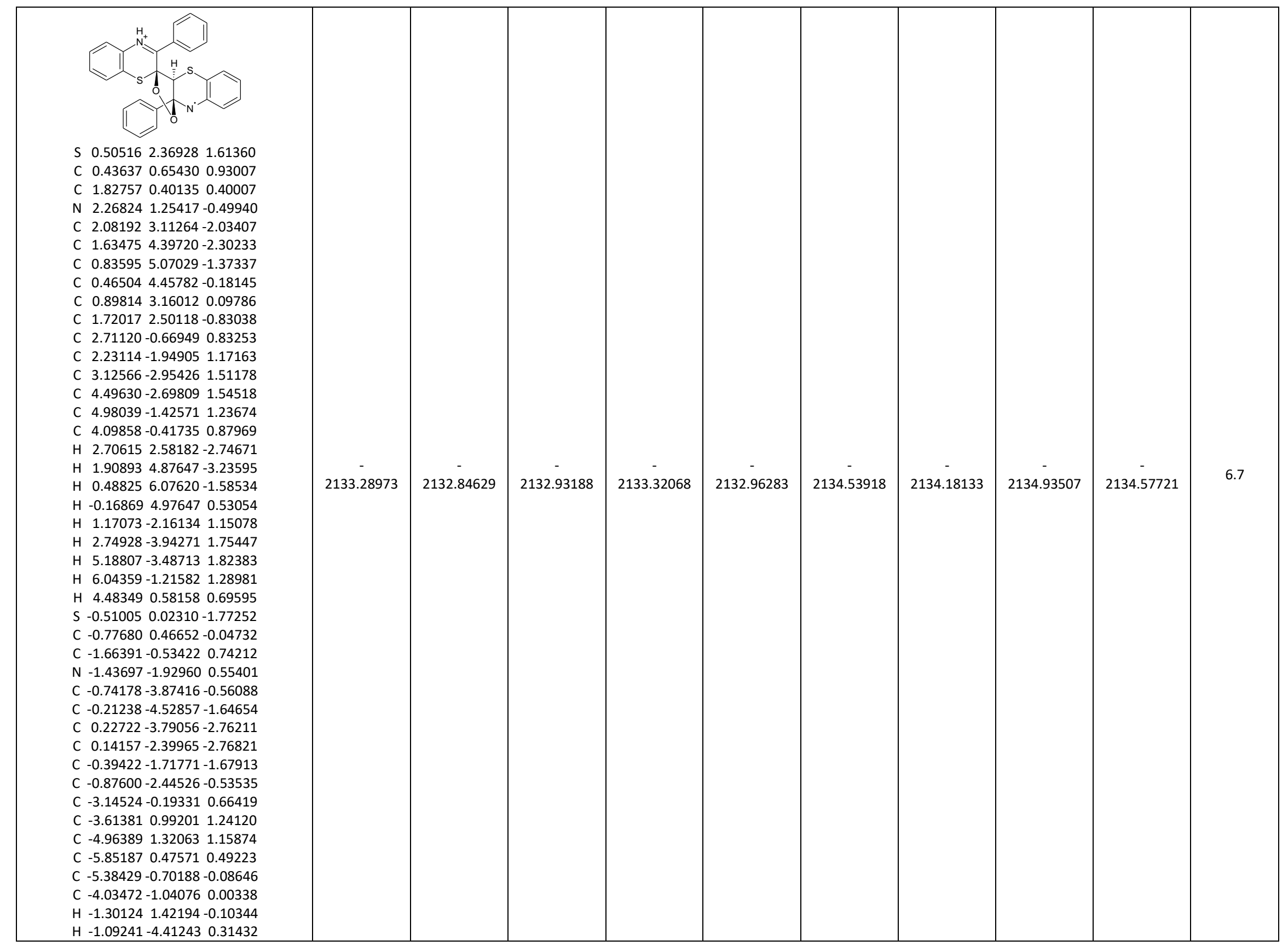




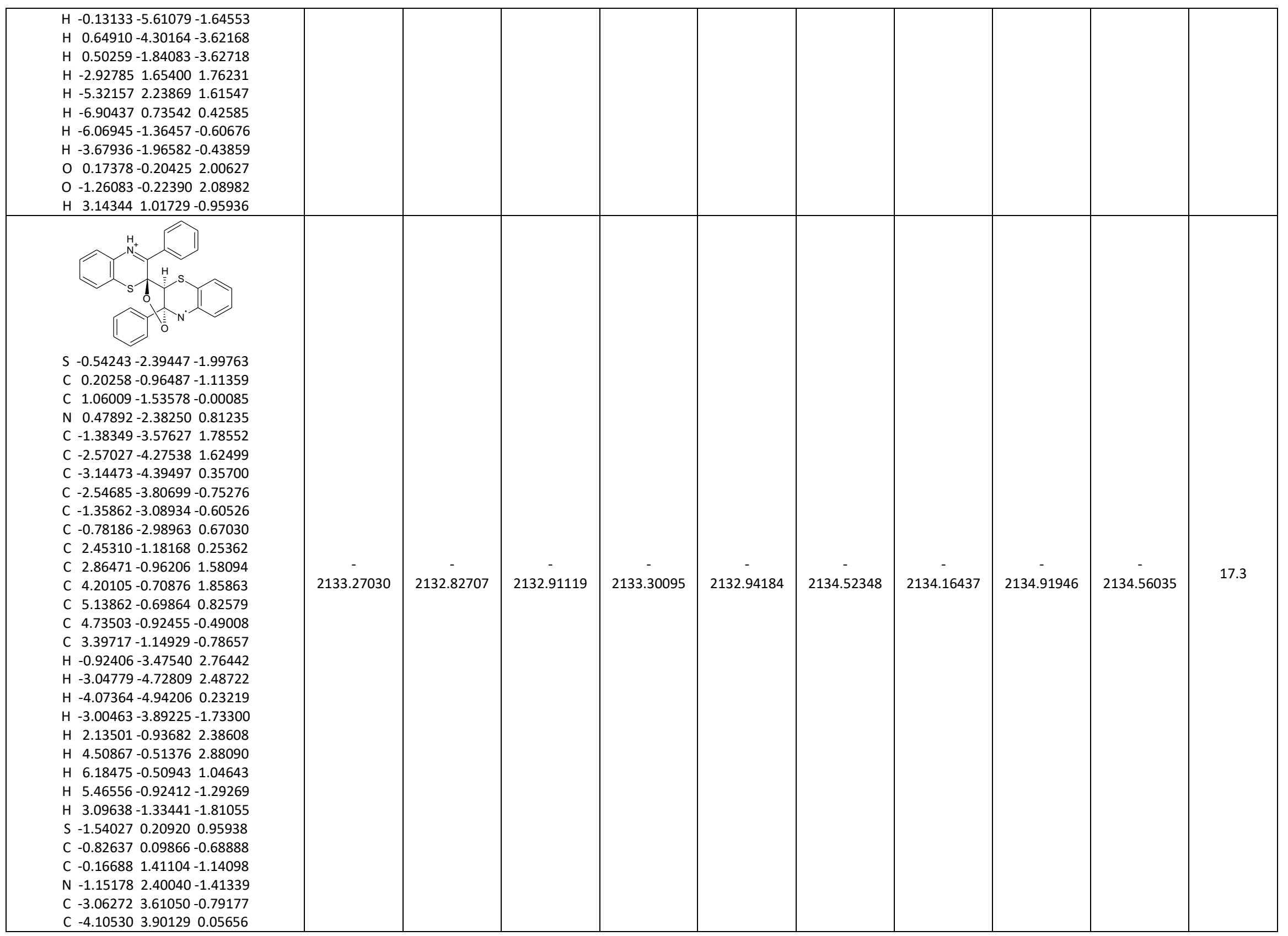




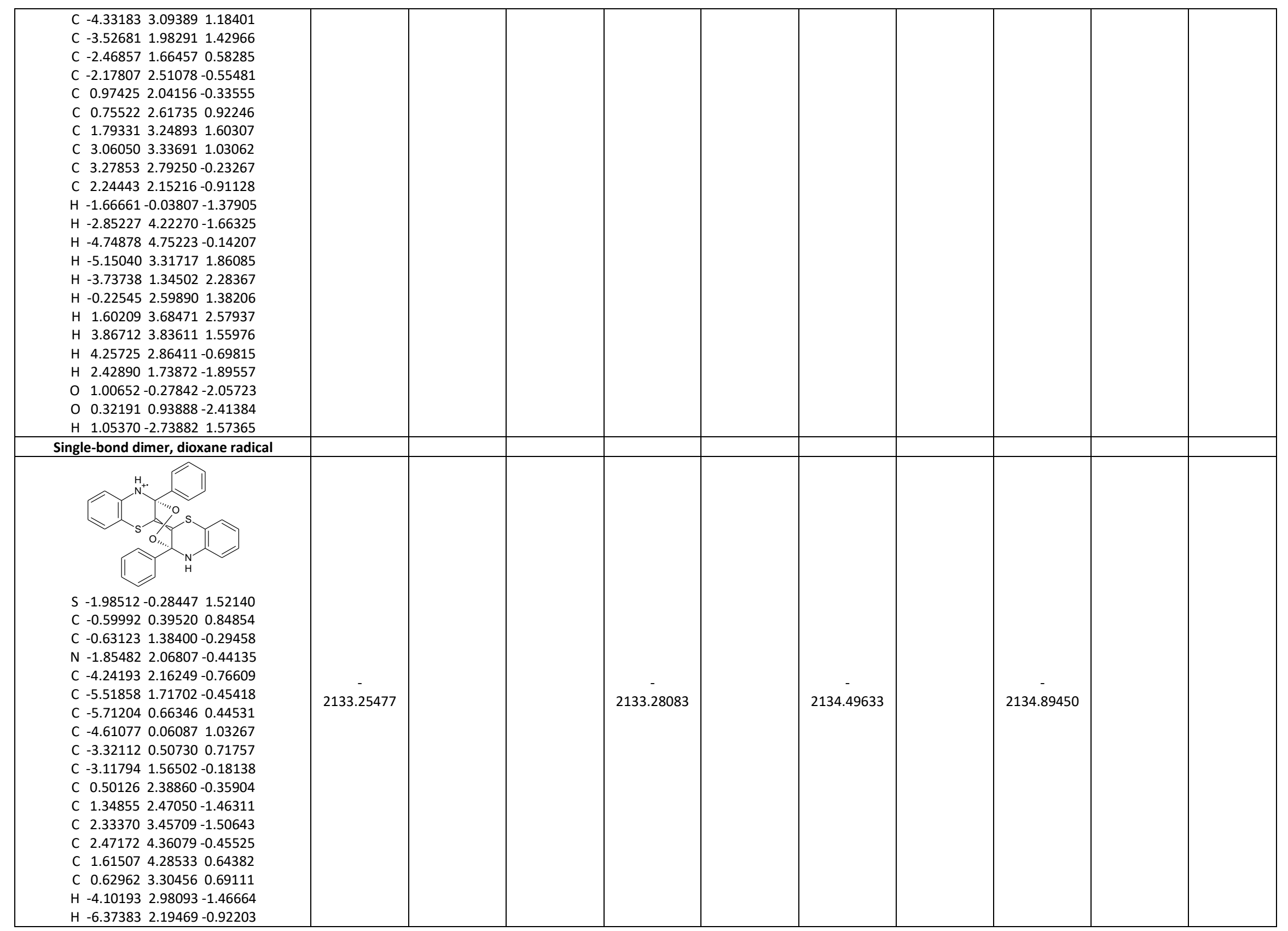




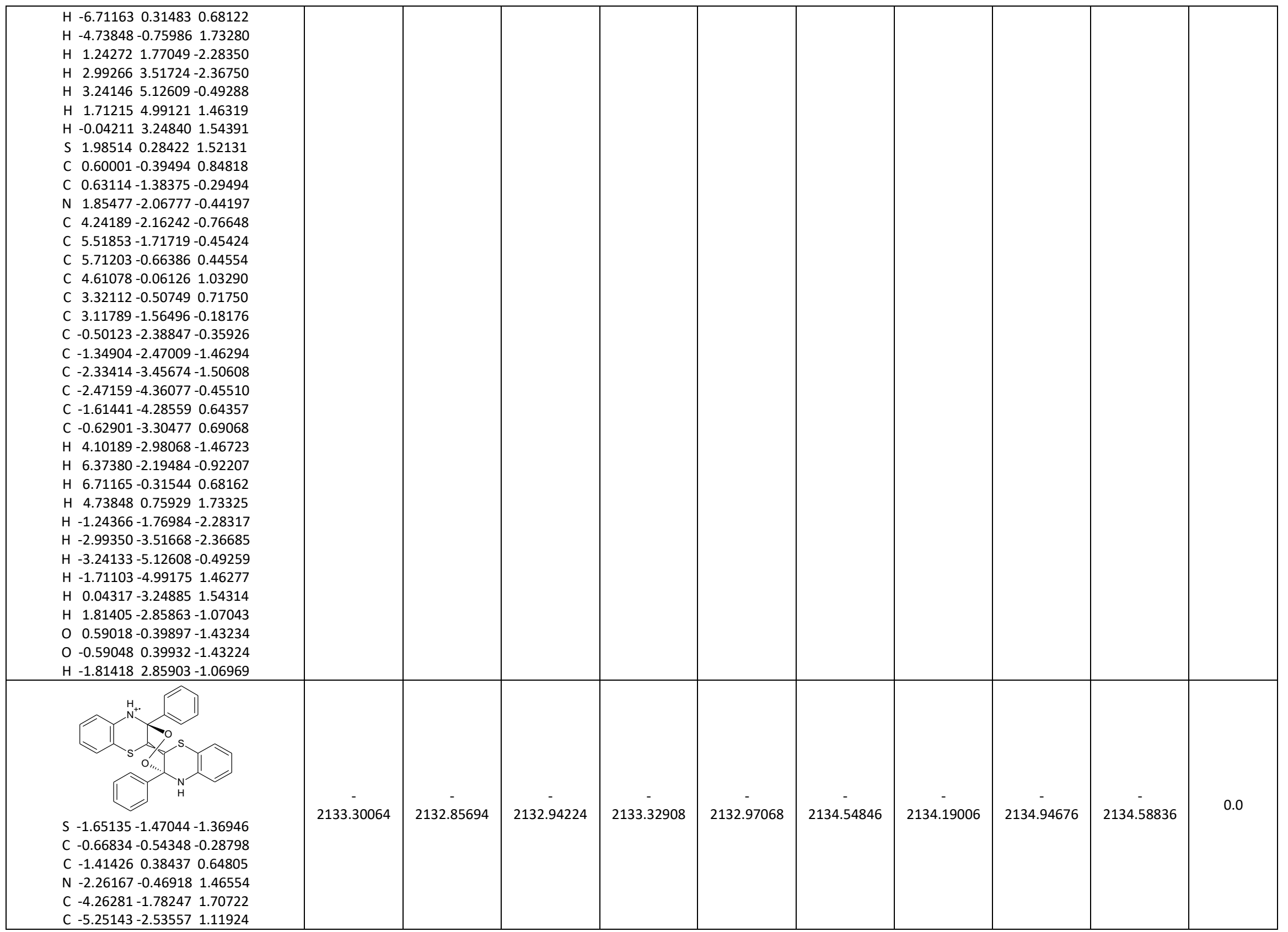




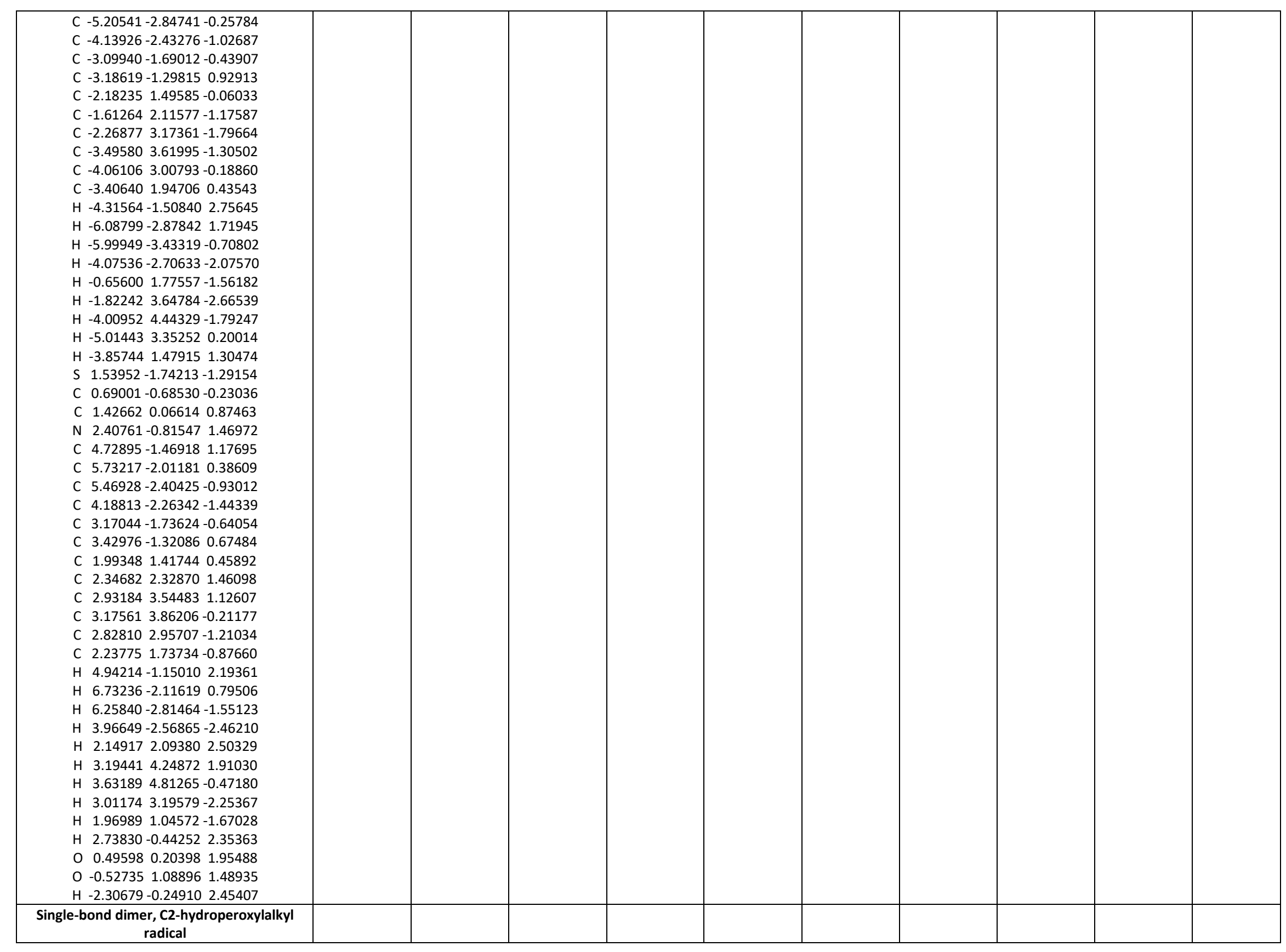




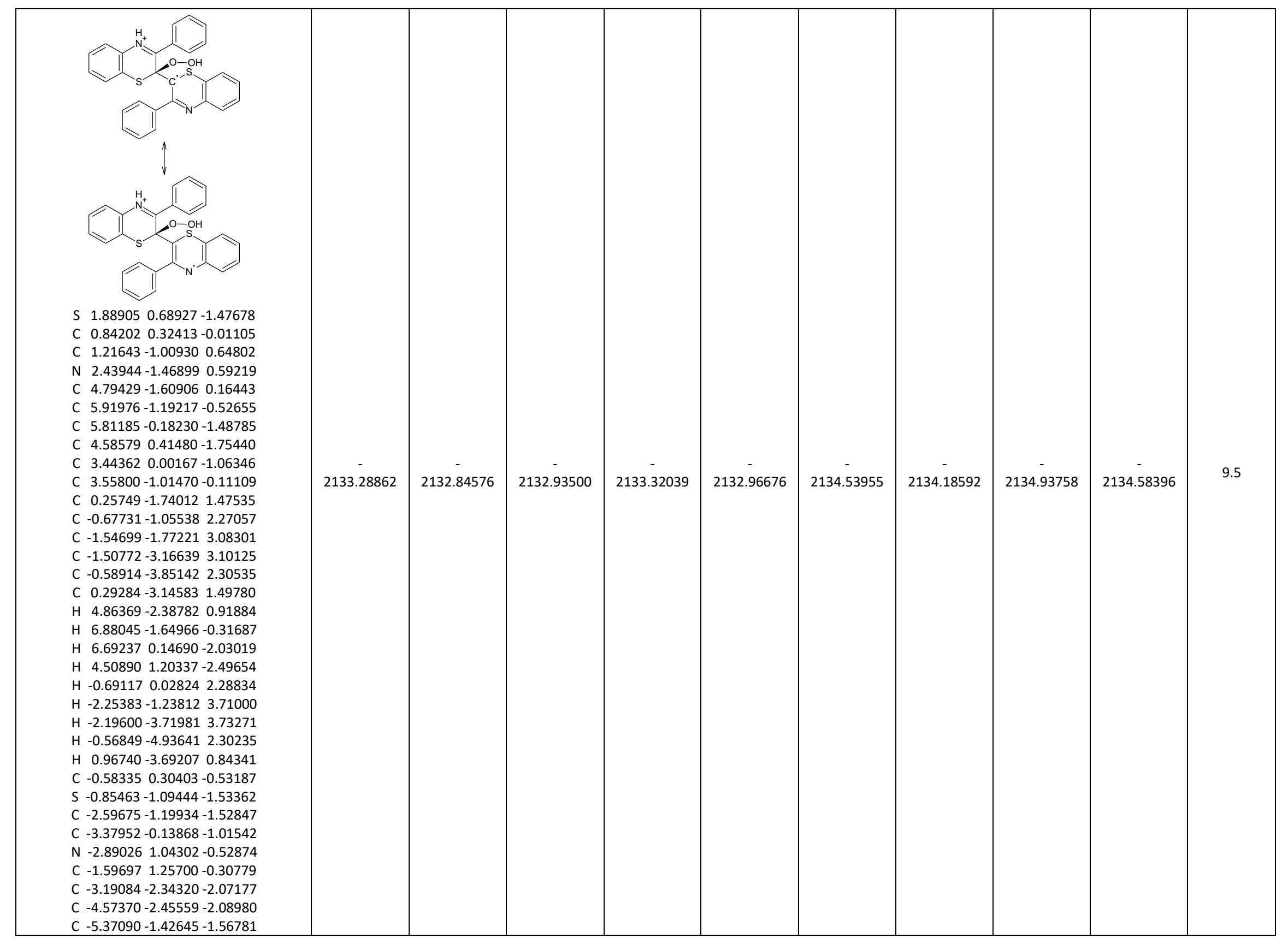




\begin{tabular}{|c|c|c|c|c|c|c|c|c|c|c|}
\hline $\begin{array}{llll}\text { C } & -4.78351 & -0.28932 & -1.04555 \\
\text { C } & -1.30582 & 2.62895 & 0.20006 \\
\text { C } & -0.43493 & 3.47746 & -0.49109 \\
\text { C } & -0.23111 & 4.78306 & -0.05127 \\
\text { C } & -0.88798 & 5.24836 & 1.08787 \\
\text { C } & -1.76330 & 4.40772 & 1.77605 \\
\text { C } & -1.98154 & 3.10785 & 1.32675 \\
\text { H } & -2.57028 & -3.14142 & -2.46921 \\
\text { H } & -5.03376 & -3.34564 & -2.50725 \\
\text { H } & -6.45242 & -1.51938 & -1.58116 \\
\text { H } & -5.38246 & 0.52486 & -0.64916 \\
\text { H } & 0.07221 & 3.12372 & -1.38449 \\
H & 0.43834 & 5.43821 & -0.60127 \\
\text { H } & -0.72390 & 6.26490 & 1.43379 \\
\text { H } & -2.28152 & 4.76657 & 2.66071 \\
\text { H } & -2.67312 & 2.45445 & 1.85069 \\
\text { O } & 0.92443 & 1.30382 & 1.00372 \\
\text { O } & 2.26140 & 1.32905 & 1.49653 \\
\text { H } & 2.57715 & 2.17916 & 1.14696 \\
\text { H } & 2.62073 & -2.28021 & 1.18118 \\
\end{array}$ & & & & & & & & & & \\
\hline 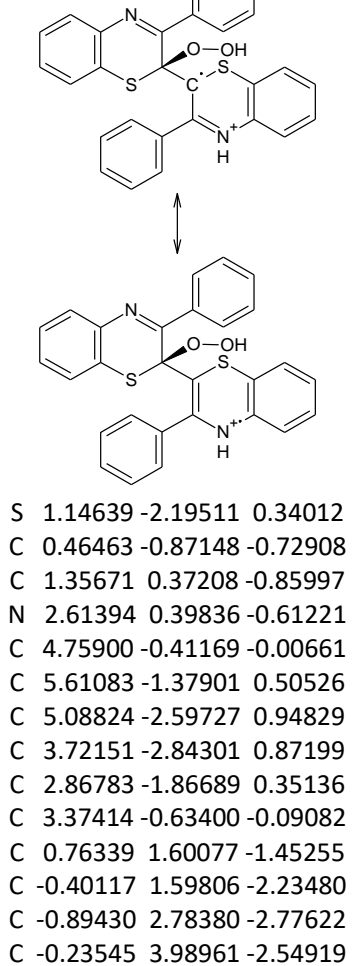 & $\begin{array}{c}- \\
- \\
2133.30164\end{array}$ & 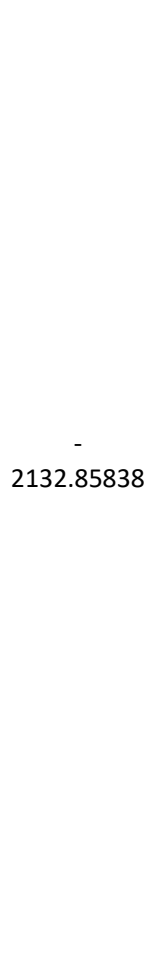 & 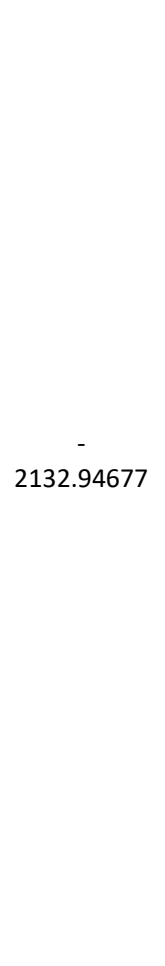 & . & . & $\begin{array}{c}- \\
2134.55554\end{array}$ & $\begin{array}{c}- \\
2134.20067\end{array}$ & $\begin{array}{c}- \\
2134.95405\end{array}$ & $\begin{array}{c}- \\
2134.59917\end{array}$ & 0.0 \\
\hline
\end{tabular}




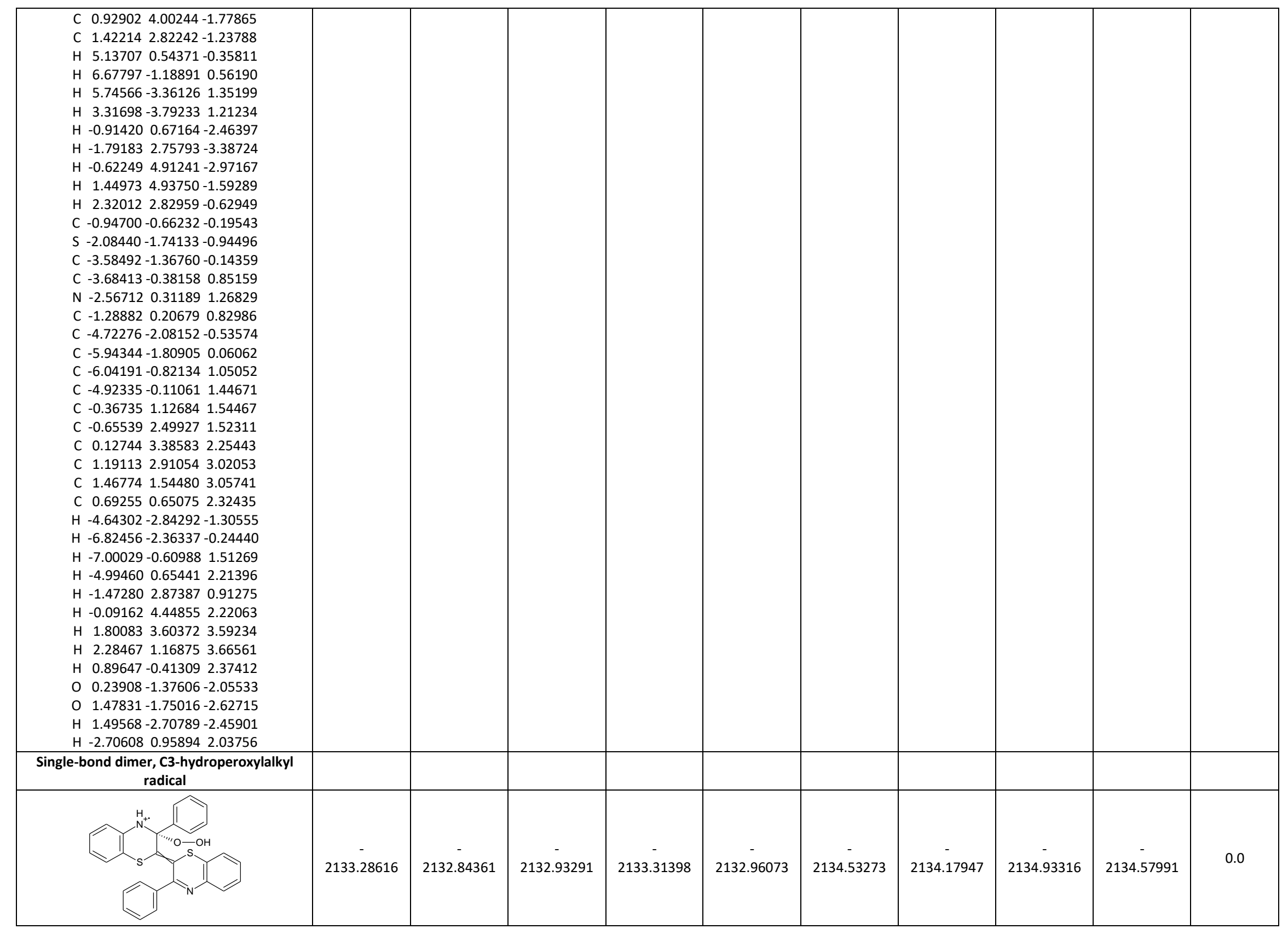

S133 
S $1.76714-1.147991 .01775$

$\begin{array}{llll}\text { C } & 0.92268 & 0.22849 & 0.37361\end{array}$

C $1.740931 .35385-0.09493$

N $2.931911 .22558-0.59641$

$\begin{array}{llll}\text { C } 4.85672 & 0.01683-1.30787\end{array}$

C $5.69761-1.07271-1.19427$

C $5.36801-2.13782-0.33673$

C $4.18190-2.127120 .37491$

C $3.30064-1.045230 .22136$

C $3.646220 .06125-0.58919$

C 1.246242 .742830 .05010

C 0.592273 .143041 .22400

C 0.199964 .467721 .38393

$\begin{array}{lllll}C & 0.44365 & 5.39775 & 0.37234\end{array}$

C $1.099125 .00359-0.79497$

C $1.510203 .68434-0.95267$

H $5.111630 .87007-1.92828$

H $6.62880-1.09812-1.75101$

H $6.04310-2.98153-0.23617$

H $3.91703-2.958991 .02074$

H 0.398492 .420242 .01253

H - 0.295354 .775272 .29997

H 0.126716 .429210 .49558

H $1.290985 .72578-1.58290$

H $2.024993 .36879-1.85469$

S - $-1.291811 .35798-0.63958$

C -0.473800 .170360 .28292$

C $-1.23581-0.992900 .92569$

N $-2.63573-0.676821 .15624$

C $-4.82280-0.422650 .14274$

C $-5.639660 .19522-0.79141$

C -5.11982 $1.12731-1.70040$

C $-3.773261 .44305-1.65891$

C $-2.945110 .82305-0.71044$

$\begin{array}{lll}C & -3.45598-0.11671 & 0.19725\end{array}$

C $-1.06706-2.310370 .17274$

C - $-1.23323-3.521200 .85531$

C $-1.17417-4.725070 .15910$

C $-0.95973-4.73105-1.21914$

C $-0.80412-3.52585-1.90029$

C $-0.85749-2.31758-1.20815$

H - $-5.23273-1.148170 .83978$

H $-6.69510-0.05701-0.82052$

H $-5.764721 .59899-2.43385$

H $-3.351792 .16615-2.35123$

H - $-1.39845-3.527081 .92822$

H $-1.29485-5.660680 .69696$

H $-0.91504-5.67194-1.75948$

H $-0.63820-3.52113-2.97331$

H $-0.72486-1.38740-1.75371$

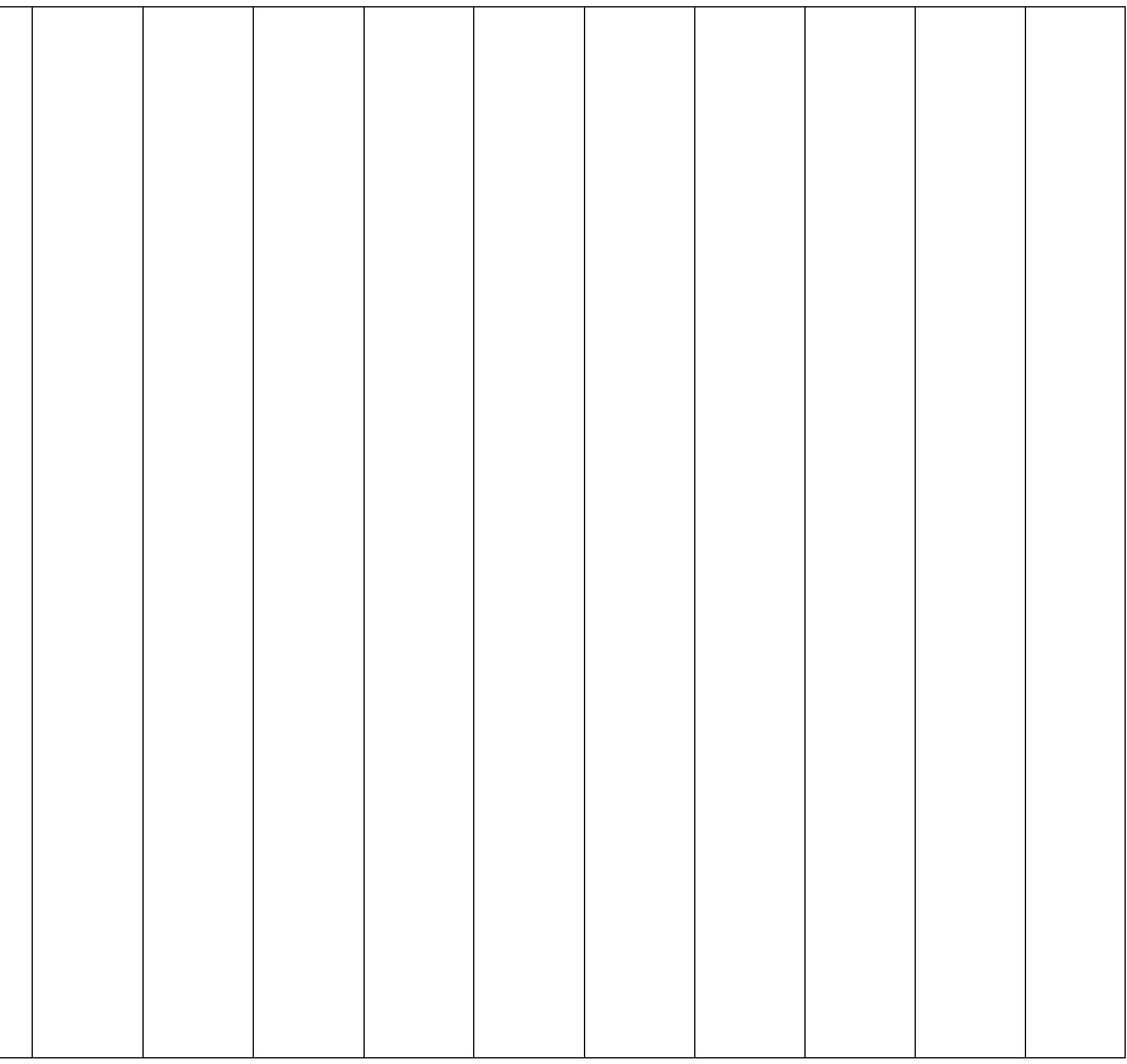




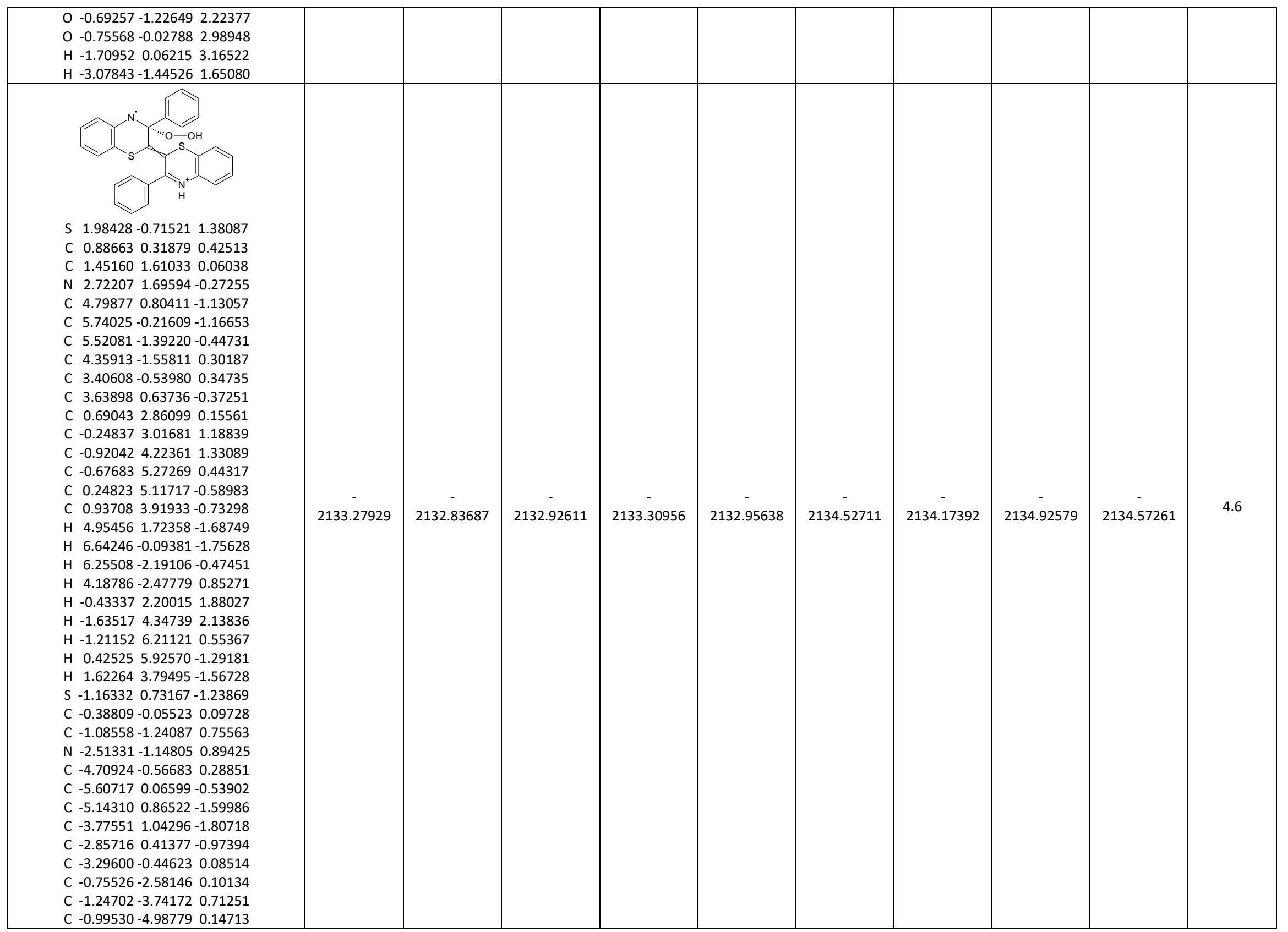




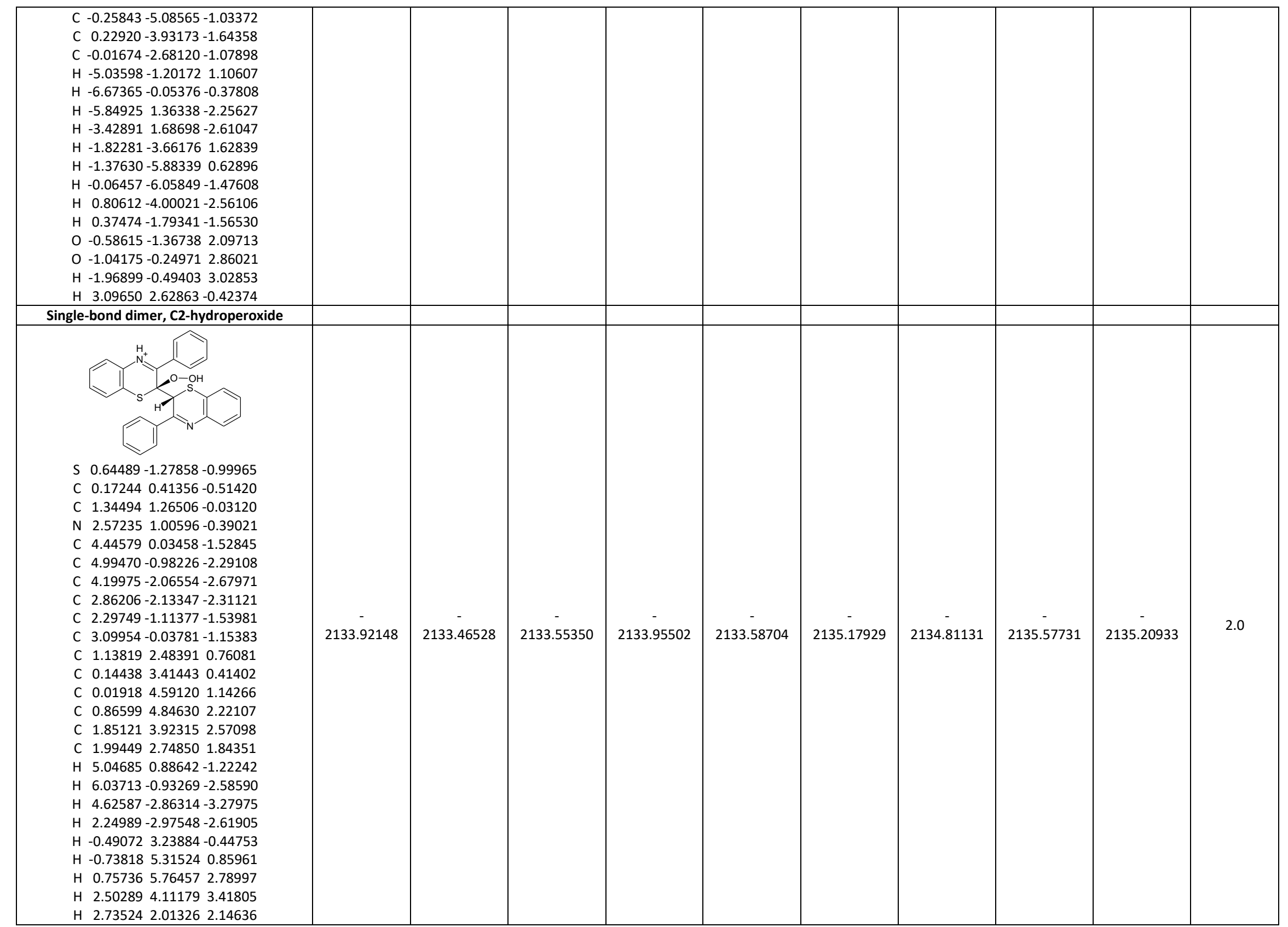




\begin{tabular}{|c|c|c|c|c|c|c|c|c|c|c|}
\hline $\begin{array}{llll}\text { C } & -0.93763 & 0.32032 & 0.57910 \\
\text { S } & -0.29198 & -0.07124 & 2.22698 \\
\text { C } & -0.34378 & -1.82820 & 2.18126 \\
\text { C } & -1.26650 & -2.48607 & 1.34801 \\
\text { N } & -2.19032 & -1.83040 & 0.54042 \\
\text { C } & -2.10276 & -0.59441 & 0.20266 \\
\text { C } & 0.49303 & -2.55816 & 3.02596 \\
\text { C } & 0.42900 & -3.94910 & 3.03567 \\
\text { C } & -0.46655 & -4.61598 & 2.19700 \\
\text { C } & -1.31141 & -3.88712 & 1.36949 \\
\text { C } & -3.19762 & -0.02940 & -0.62132 \\
\text { C } & -3.90925 & -0.88745 & -1.47511 \\
\text { C } & -4.95779 & -0.40720 & -2.24977 \\
\text { C } & -5.32448 & 0.93806 & -2.17760 \\
\text { C } & -4.63060 & 1.79719 & -1.32811 \\
\text { C } & -3.56929 & 1.32103 & -0.56196 \\
\text { H } & 1.19858 & -2.03868 & 3.66847 \\
\text { H } & 1.08428 & -4.51027 & 3.69493 \\
\text { H } & -0.51232 & -5.70059 & 2.19808 \\
\text { H } & -2.02777 & -4.38286 & 0.72098 \\
\text { H } & -3.61865 & -1.93168 & -1.52576 \\
\text { H } & -5.48893 & -1.08213 & -2.91464 \\
\text { H } & -6.14576 & 1.31363 & -2.78094 \\
\text { H } & -4.91495 & 2.84279 & -1.25722 \\
\text { H } & -3.06350 & 2.01390 & 0.10358 \\
\text { O } & -0.45576 & 1.11874 & -1.57262 \\
\text { O } & 0.49400 & 1.31208 & -2.61851 \\
\text { H } & 0.18897 & 0.66997 & -3.28137 \\
\text { H } & -1.31886 & 1.33624 & 0.68952 \\
\text { H } & 3.25876 & 1.70444 & -0.10774\end{array}$ & & & & & & & & & & \\
\hline 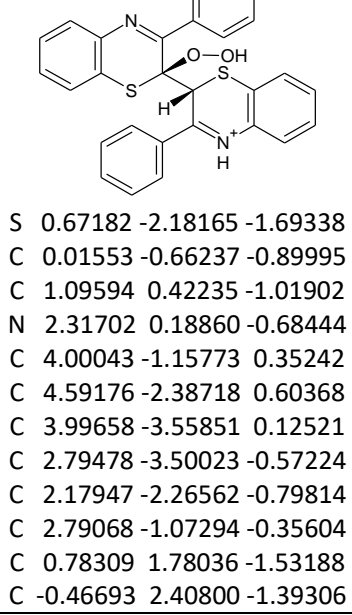 & $\begin{array}{c}- \\
2133.92288\end{array}$ & $\begin{array}{c}- \\
2133.46689\end{array}$ & $\begin{array}{c}- \\
2133.55369\end{array}$ & $\begin{array}{c}- \\
2133.95732\end{array}$ & $\begin{array}{c}- \\
2133.58812\end{array}$ & $\begin{array}{c}- \\
2135.18402\end{array}$ & $\begin{array}{c}- \\
2134.81482\end{array}$ & $\begin{array}{c}- \\
2135.58165\end{array}$ & $\begin{array}{c}- \\
2135.21246\end{array}$ & 0.1 \\
\hline
\end{tabular}




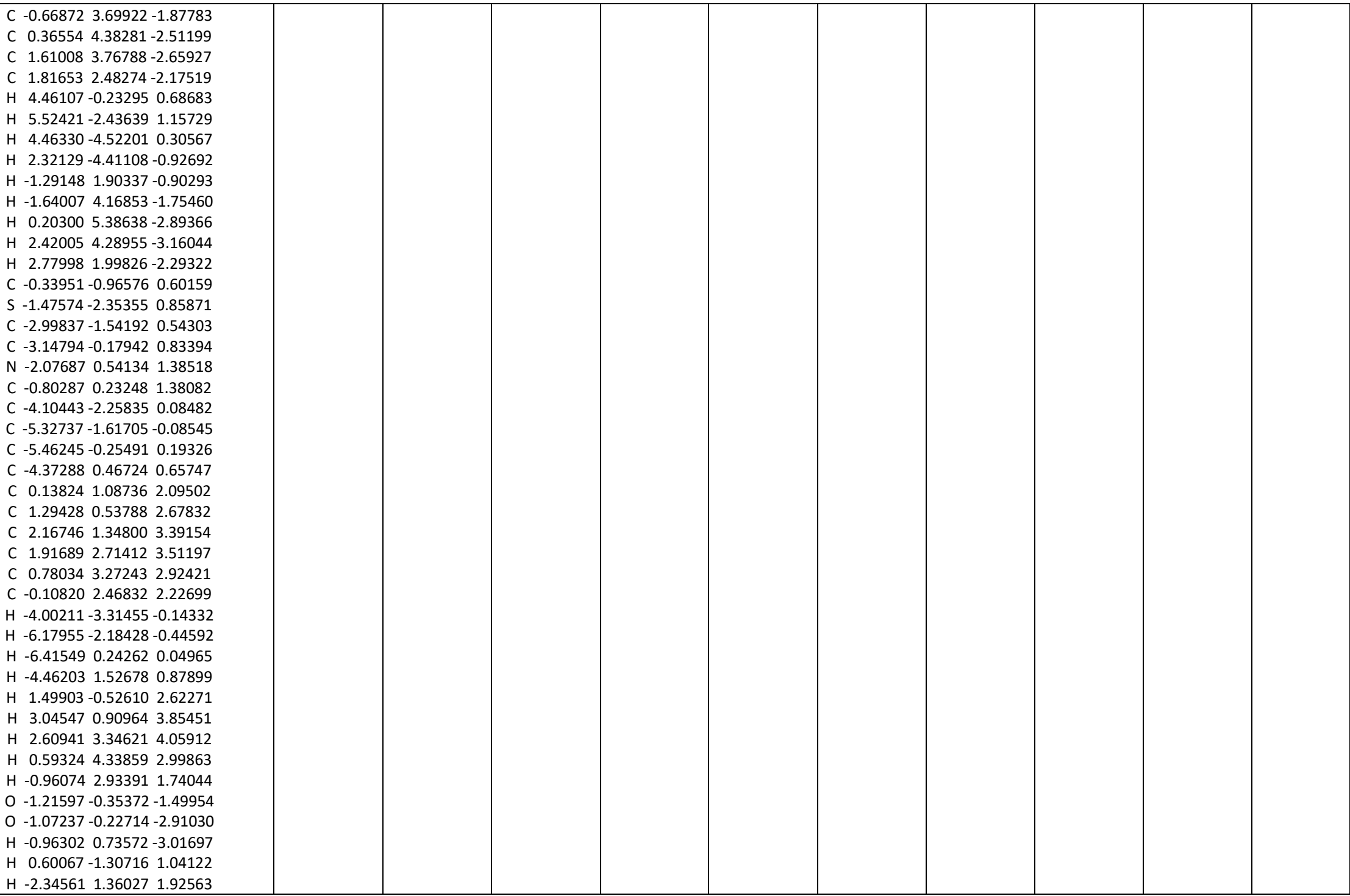




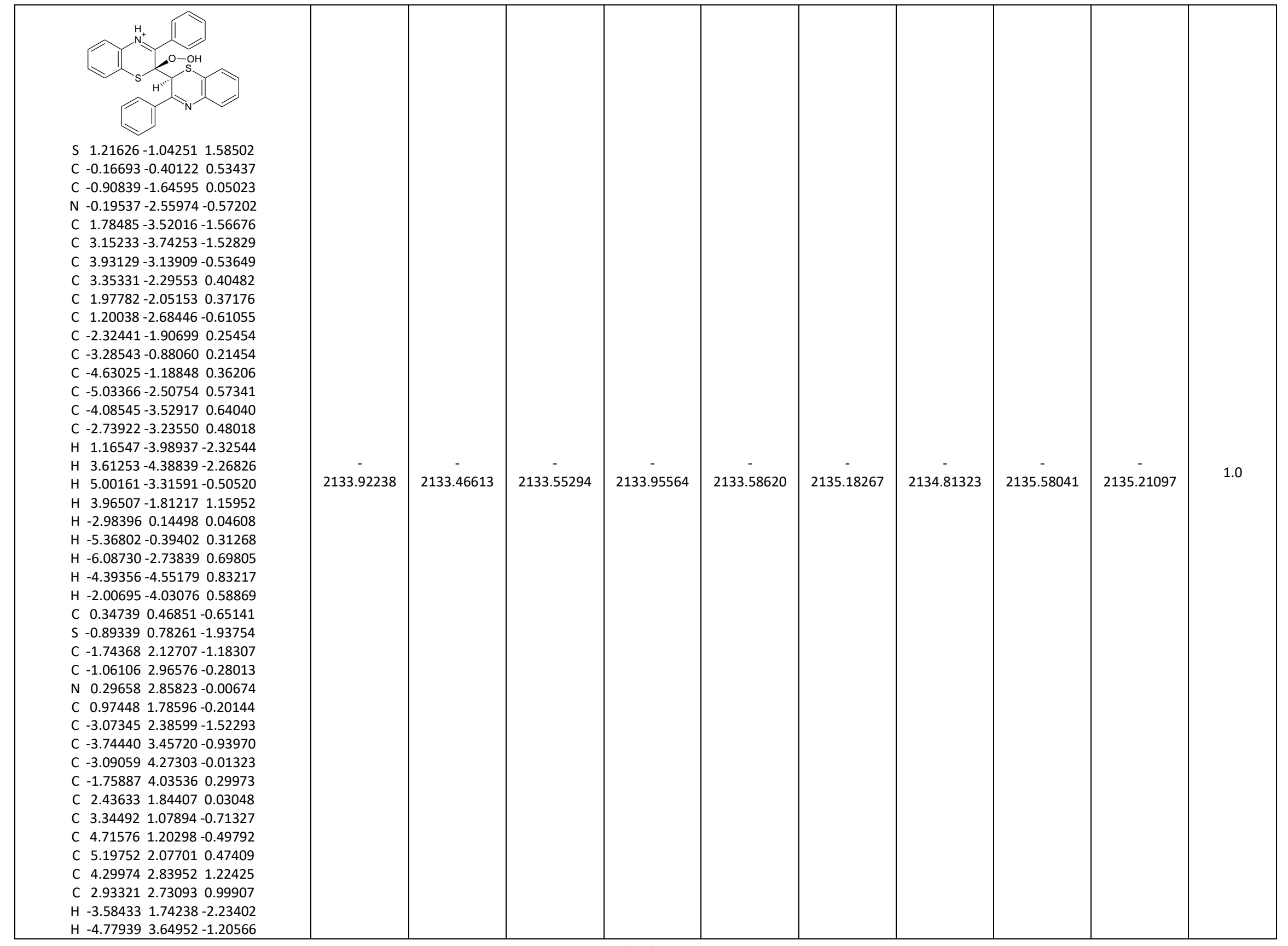




\begin{tabular}{|c|c|c|c|c|c|c|c|c|c|c|}
\hline $\begin{array}{rrrr}H & -3.61567 & 5.10291 & 0.44960 \\
H & -1.22270 & 4.67608 & 0.99369 \\
H & 3.00227 & 0.40438 & -1.49235 \\
H & 5.40651 & 0.61442 & -1.09443 \\
H & 6.26621 & 2.16480 & 0.64683 \\
H & 4.66725 & 3.51922 & 1.98758 \\
H & 2.23002 & 3.32249 & 1.57624 \\
\text { O } & -0.95486 & 0.44403 & 1.30881 \\
O & -1.36083 & -0.22674 & 2.50592 \\
H & -1.03122 & 0.39451 & 3.17609 \\
H & 1.10095 & -0.13196 & -1.16509 \\
H & -0.71497 & -3.31355 & -1.01554 \\
\end{array}$ & & & & & & & & & & \\
\hline 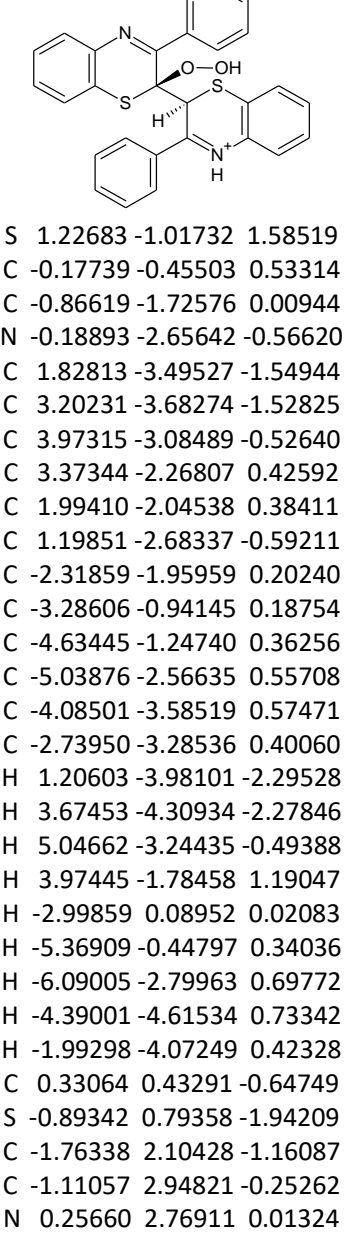 & $\begin{array}{c}- \\
2133.92422\end{array}$ & $\begin{array}{c}- \\
2133.46797\end{array}$ & $\begin{array}{c}- \\
2133.55456\end{array}$ & - & $\begin{array}{c}- \\
2133.58718\end{array}$ & $\begin{array}{c}- \\
2135.18428\end{array}$ & $\begin{array}{c}- \\
2134.81461\end{array}$ & $\begin{array}{c}- \\
2135.58224\end{array}$ & $\stackrel{-}{-}$ & 0.0 \\
\hline
\end{tabular}




\begin{tabular}{|c|c|c|c|c|c|c|c|c|c|c|}
\hline $\begin{array}{lrrrr}C & 0.99106 & 1.70623 & -0.19041 \\
\text { C } & -3.09073 & 2.37453 & -1.49814 \\
\text { C } & -3.74890 & 3.45765 & -0.92340 \\
\text { C } & -3.09498 & 4.28087 & -0.00458 \\
\text { C } & -1.77118 & 4.03150 & 0.32864 \\
\text { C } & 2.42650 & 1.78952 & 0.04944 \\
\text { C } & 3.32350 & 1.08205 & -0.76851 \\
\text { C } & 4.69259 & 1.22069 & -0.57846 \\
\text { C } & 5.18062 & 2.03580 & 0.44149 \\
\text { C } & 4.29502 & 2.72454 & 1.27291 \\
\text { C } & 2.92705 & 2.61296 & 1.07588 \\
\text { H } & -3.60533 & 1.73336 & -2.20727 \\
\text { H } & -4.78195 & 3.65514 & -1.19118 \\
\text { H } & -3.61466 & 5.11805 & 0.44873 \\
\text { H } & -1.24765 & 4.66708 & 1.03689 \\
\text { H } & 2.96513 & 0.46131 & -1.58274 \\
\text { H } & 5.37897 & 0.68896 & -1.22935 \\
\text { H } & 6.25130 & 2.13022 & 0.59424 \\
\text { H } & 4.67172 & 3.34136 & 2.08229 \\
\text { H } & 2.25189 & 3.11945 & 1.75978 \\
\text { O } & -0.98289 & 0.40404 & 1.29843 \\
\text { O } & -1.36577 & -0.21236 & 2.52243 \\
\text { H } & -2.23966 & -0.58212 & 2.29740 \\
\text { H } & 1.07981 & -0.17293 & -1.16391 \\
\text { H } & 0.74506 & 3.60751 & 0.31972 \\
\end{array}$ & & & & & & & & & & \\
\hline 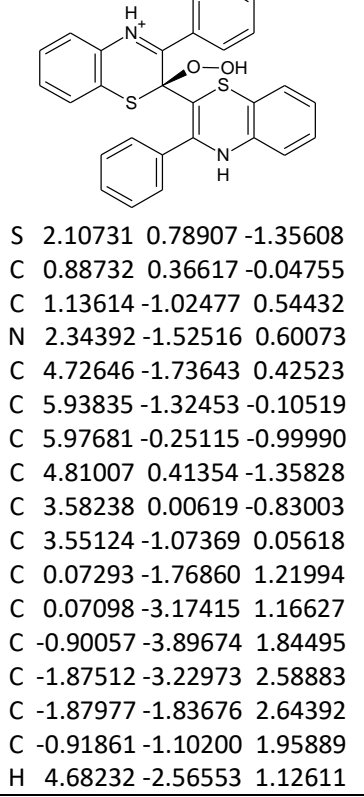 & $\begin{array}{c}- \\
2133.92388\end{array}$ & $\stackrel{-}{-}$ & $\begin{array}{c}- \\
2133.55477\end{array}$ & $\begin{array}{c}- \\
- \\
2133.95699\end{array}$ & $\begin{array}{c}- \\
- \\
- \\
2133.58788\end{array}$ & $\begin{array}{c}- \\
- \\
2135.18208\end{array}$ & $\begin{array}{c}- \\
2134.81297\end{array}$ & $\begin{array}{c}- \\
2135.58059\end{array}$ & $\begin{array}{c}- \\
2135.21148\end{array}$ & 0.7 \\
\hline
\end{tabular}




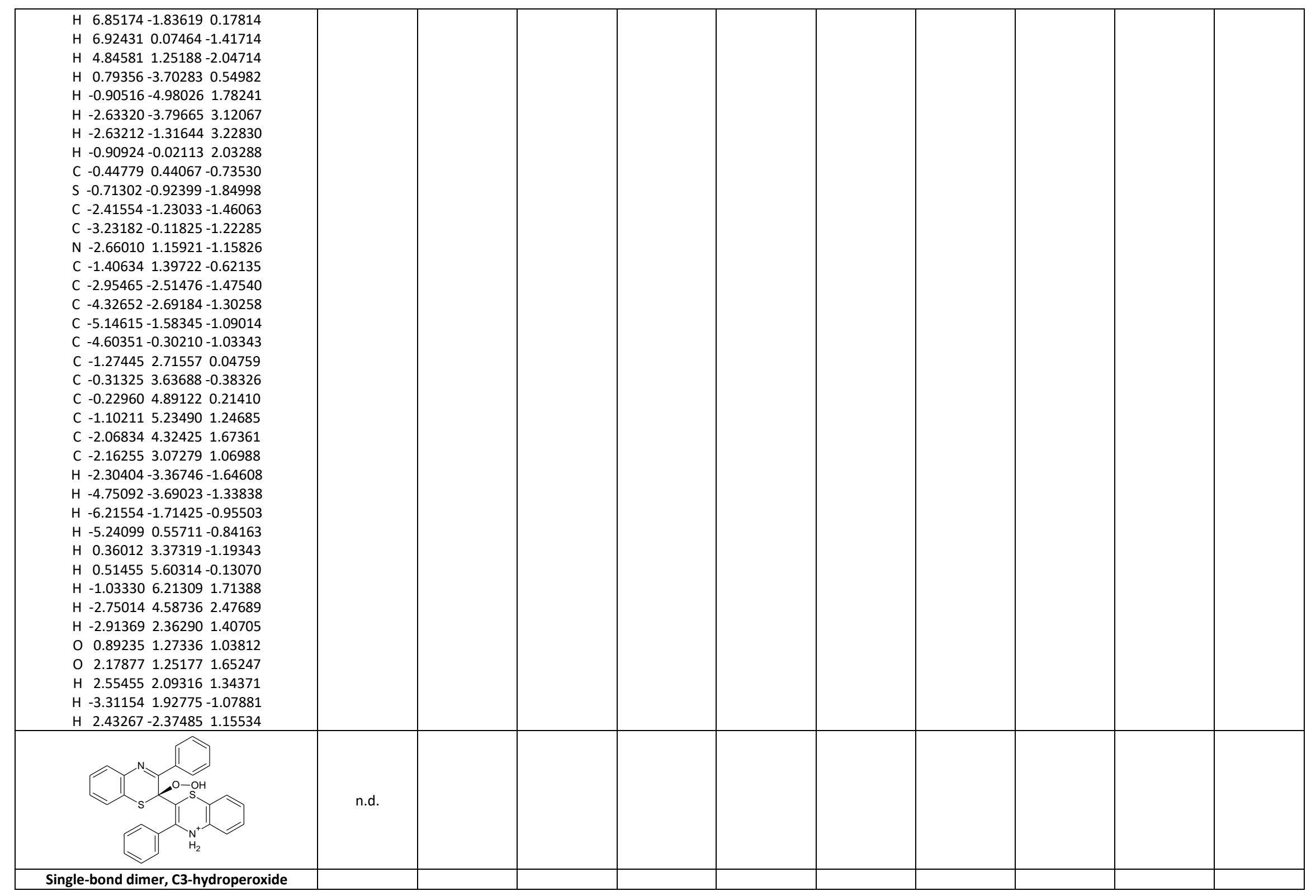




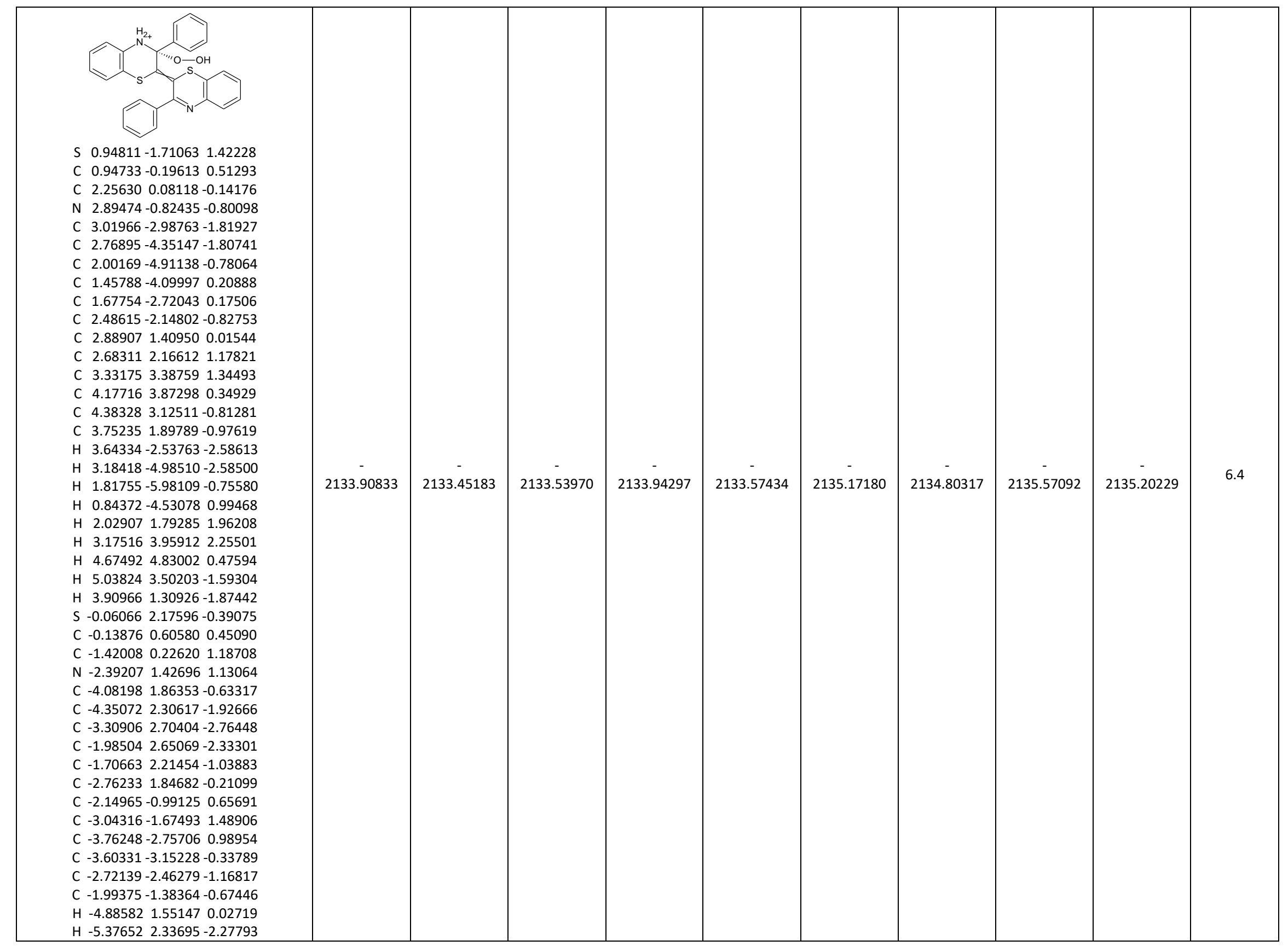




\begin{tabular}{|c|c|c|c|c|c|c|c|c|c|c|}
\hline $\begin{array}{llll}H & -3.52649 & 3.04571 & -3.77145 \\
H & -1.17367 & 2.93806 & -2.99380 \\
H & -3.16819 & -1.37959 & 2.52620 \\
H & -4.44656 & -3.29154 & 1.64136 \\
H & -4.16619 & -3.99652 & -0.72465 \\
H & -2.59426 & -2.76517 & -2.20300 \\
H & -1.30024 & -0.85852 & -1.32401 \\
\text { H } & -3.22119 & 1.16914 & 1.67465 \\
O & -1.19888 & 0.01072 & 2.55147 \\
O & -0.56433 & 1.19747 & 3.06177 \\
\text { H } & -0.96778 & 1.24129 & 3.94459 \\
H & -1.92461 & 2.19403 & 1.63993 \\
\end{array}$ & & & & & & & & & & \\
\hline 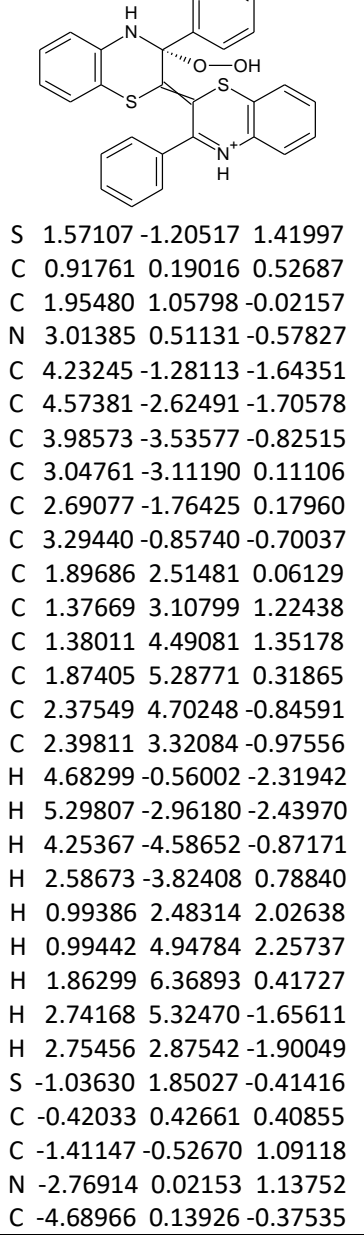 & $\begin{array}{c}- \\
2133.92514\end{array}$ & $\begin{array}{c}- \\
2133.46843\end{array}$ & $\begin{array}{c}- \\
2133.55589\end{array}$ & $\begin{array}{c}- \\
2133.95743\end{array}$ & $\begin{array}{c}- \\
\\
2133.58817\end{array}$ & . & $\begin{array}{c}- \\
2134.81314\end{array}$ & $\begin{array}{c}- \\
2135.58174\end{array}$ & $\begin{array}{c}- \\
2135.21248\end{array}$ & 0.0 \\
\hline
\end{tabular}




\begin{tabular}{|c|c|c|c|c|c|c|c|c|c|c|}
\hline $\begin{array}{llll}\text { C } & -5.27096 & 0.66726 & -1.52413 \\
\text { C } & -4.54175 & 1.50829 & -2.36606 \\
\text { C } & -3.22393 & 1.82750 & -2.05037 \\
\text { C } & -2.65349 & 1.32098 & -0.88267 \\
\text { C } & -3.37533 & 0.47492 & -0.03829 \\
\text { C } & -1.39881 & -1.92684 & 0.49048 \\
\text { C } & -1.75237 & -3.03089 & 1.27353 \\
\text { C } & -1.83964 & -4.29500 & 0.69556 \\
\text { C } & -1.58922 & -4.46607 & -0.66586 \\
\text { C } & -1.25198 & -3.36435 & -1.44946 \\
\text { C } & -1.15761 & -2.09830 & -0.87489 \\
\text { H } & -5.25444 & -0.52757 & 0.27106 \\
\text { H } & -6.29629 & 0.40749 & -1.76934 \\
\text { H } & -4.99190 & 1.90755 & -3.26926 \\
\text { H } & -2.64074 & 2.47387 & -2.69998 \\
\text { H } & -1.94743 & -2.90783 & 2.33420 \\
\text { H } & -2.10319 & -5.14864 & 1.31323 \\
\text { H } & -1.65860 & -5.45319 & -1.11339 \\
\text { H } & -1.05883 & -3.48660 & -2.51116 \\
\text { H } & -0.88605 & -1.24906 & -1.49601 \\
\text { H } & -3.35897 & -0.65092 & 1.62026 \\
\text { O } & -1.03695 & -0.67198 & 2.45810 \\
\text { O } & -1.03327 & 0.61387 & 3.07544 \\
\text { H } & -1.95123 & 0.91302 & 2.92030 \\
\text { H } & 3.73937 & 1.14309 & -0.90577 \\
\end{array}$ & & & & & & & & & & \\
\hline Single-bond dimer, dioxolane & & & & & & & & & & \multirow[b]{2}{*}{11.2} \\
\hline $\begin{array}{lrrr}S & -1.69362 & -1.64736 & -0.73646 \\
C & -1.12437 & -0.13311 & 0.10596 \\
C & -2.15390 & 0.98468 & -0.10907 \\
\text { N } & -3.41376 & 0.77343 & 0.00563 \\
C & -5.27731 & -0.52624 & 0.73788 \\
C & -5.91814 & -1.74090 & 0.93500 \\
C & -5.26411 & -2.93456 & 0.61625 \\
C & -3.96315 & -2.91176 & 0.12485 \\
C & -3.30788 & -1.69048 & -0.04517 \\
C & -3.96438 & -0.47996 & 0.24584 \\
C & -1.70448 & 2.36296 & -0.42801 \\
C & -2.19035 & 2.98572 & -1.58349 \\
C & -1.81402 & 4.29161 & -1.88663 \\
C & -0.96169 & 4.99069 & -1.03246 \\
C & -0.48817 & 4.38139 & 0.12922 \\
C & -0.85212 & 3.07106 & 0.43035 \\
\end{array}$ & 2133.91789 & 2133.46025 & 2133.54566 & 2133.95136 & 2133.57913 & $\begin{array}{c}- \\
2135.17601\end{array}$ & 2134.80378 & $\begin{array}{c}- \\
2135.57288\end{array}$ & $\begin{array}{c}- \\
2135.20065\end{array}$ & \\
\hline
\end{tabular}




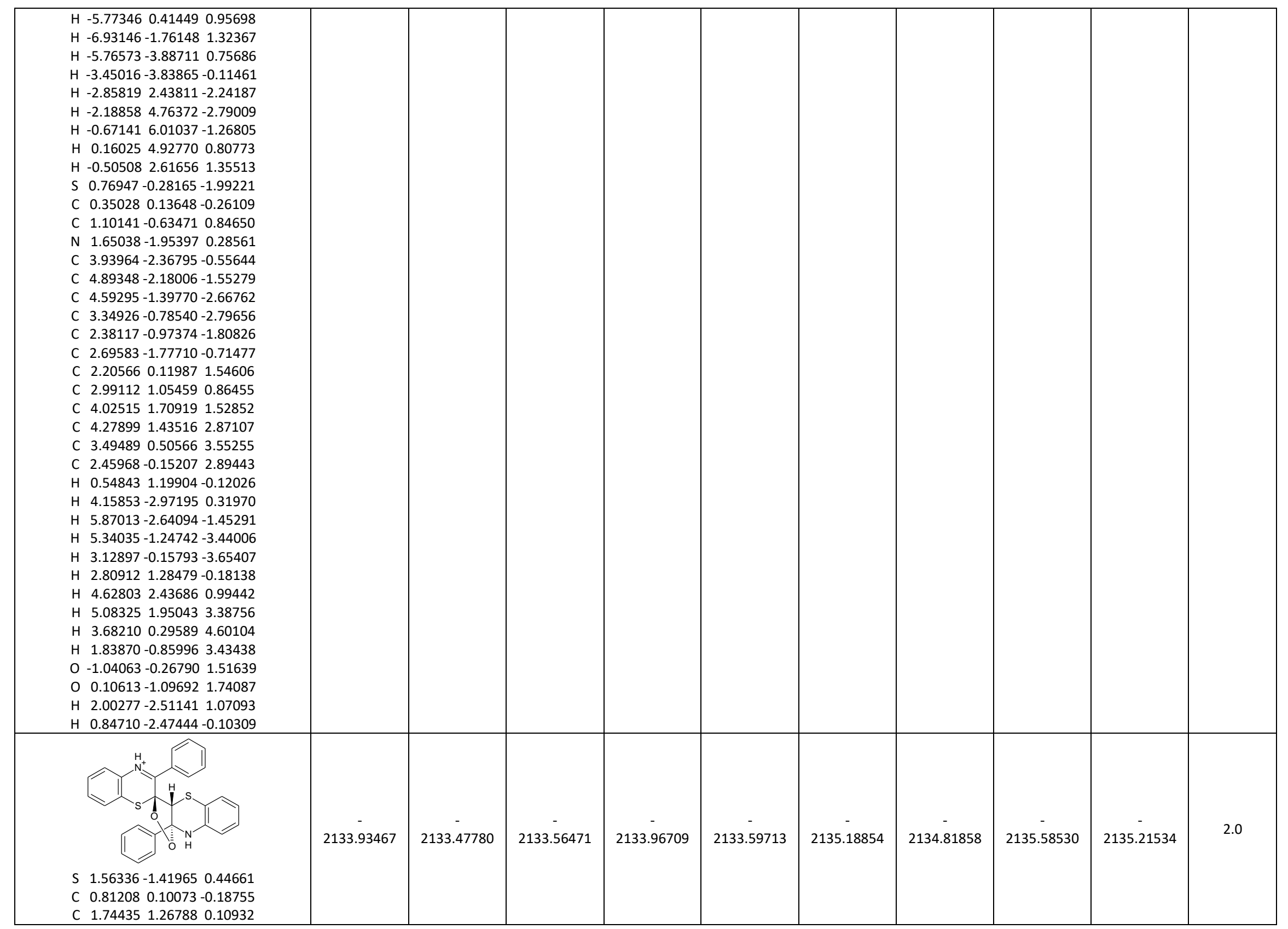

S146 
N $3.026391 .12648-0.12283$

C $5.038450 .10007-0.96186$

C $5.76450-1.04361-1.24916$

$5.19449-2.30481-1.04306$

C $3.89788-2.42835-0.56001$

C $3.15192-1.28199-0.27348$

C $3.73476-0.02555-0.47094$

C 1.307722 .582110 .58756

C $0.293533 .28946-0.07784$

$\begin{array}{llll}C & -0.06020 & 4.55961 & 0.35795\end{array}$

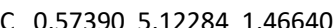

C 1.578544 .422082 .13170

C 1.957403 .158621 .69093

H $5.467221 .08597-1.11546$

H $6.77490-0.95669-1.63368$

H $5.76461-3.19993-1.27001$

H $3.45689-3.40873-0.41071$

H $-0.180042 .86649-0.95809$

H -0.83049 $5.11169-0.17092$

H $0.283506 .11048 \quad 1.81120$

H 2.067174 .855972 .99813

H 2.724762 .604382 .22411

S -1.12274-0.61965 1.85540

$\begin{array}{llll}C & -0.64194 & 0.23958 & 0.35543\end{array}$

C $-1.48346-0.09562-0.89544$

N $-1.58735-1.50738-1.10060$

C $-2.02028-3.79795-0.51851$

C - $2.11534-4.822730 .41193$

C $-1.88230-4.57170176595$

C $-1.55208-3.283332 .16760$

C $-1.46625-2.243261 .23711$

C $-1.69861-2.48783-0.12589$

C $-2.813160 .64351-0.95598$

C $-3.925460 .06305-0.33880$

C $-5.153300 .72037-0.34251$

C $-5.286151 .95470-0.97645$

C -4.18346 2.52914-1.60551

C -2.94909 $1.88185-1.59189$

H -0.823371 .288740 .59239$

H -2.19659-3.99972 -1.57213

H - $2.37011-5.823420 .07584$

H $-1.95118-5.368732 .49914$

H -1.35928-3.07181 3.21622

H -3.83944 -0.906260 .14278$

H -6.008040 .261360 .14542$

H $-6.245872 .46296-0.98572$

H $-4.279993 .48448-2.11293$

H -2.10395 $2.33651-2.09688$

O $0.66863 \quad 0.02115-1.60704$

$\begin{array}{llll}0 & -0.66654 & 0.45925 & -1.92444\end{array}$

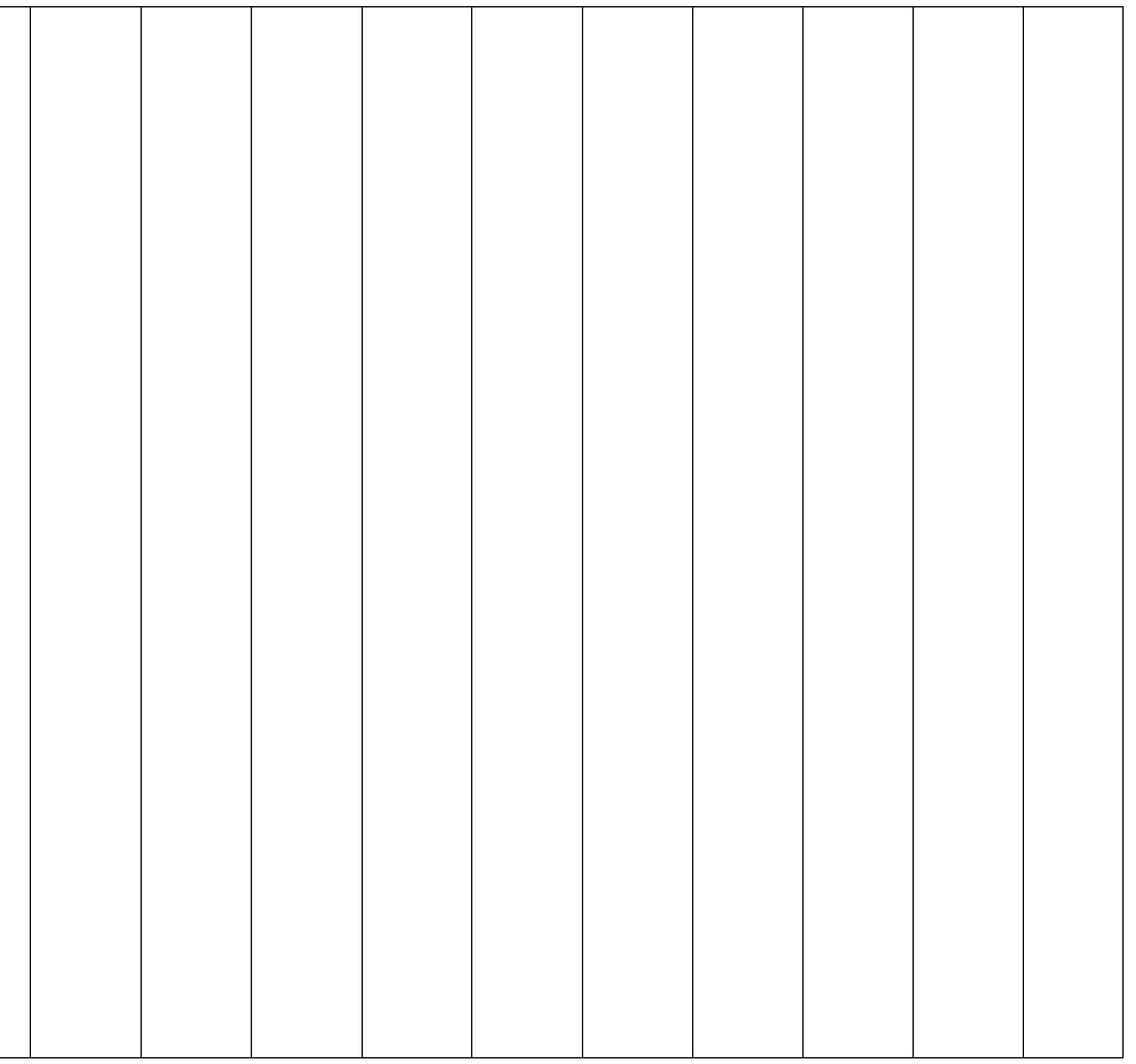




\begin{tabular}{|c|c|c|c|c|c|c|c|c|c|c|}
\hline $\begin{array}{lrrr}H & -1.98751 & -1.74797 & -1.99790 \\
\text { H } & 3.58616 & 1.97295 & -0.03921 \\
\end{array}$ & & & & & & & & & & \\
\hline $\begin{array}{llll} & \end{array}$ & $\begin{array}{c}- \\
2133.90068\end{array}$ & $\begin{array}{c}- \\
2133.44291\end{array}$ & $\begin{array}{c}- \\
2133.52768\end{array}$ & $\begin{array}{c}- \\
2133.93597\end{array}$ & $\begin{array}{c}- \\
2133.56297\end{array}$ & $\stackrel{-}{-}$ & $\begin{array}{c}- \\
- \\
2134.78816\end{array}$ & $\begin{array}{c}- \\
- \\
- \\
2135.55787\end{array}$ & $\begin{array}{c}- \\
- \\
2135.18487\end{array}$ & 21.1 \\
\hline
\end{tabular}




\begin{tabular}{|c|c|c|c|c|c|c|c|c|c|c|}
\hline $\begin{array}{lrrr}C & 2.17576 & -2.73019 & -0.30142 \\
H & 0.80359 & 1.22106 & 0.49744 \\
H & 5.01422 & 0.21906 & 1.64704 \\
H & 6.62647 & 1.00022 & -0.05439 \\
H & 5.84468 & 1.43546 & -2.38536 \\
H & 3.47455 & 1.10952 & -2.97325 \\
H & -0.31143 & -2.85721 & 2.01245 \\
H & -0.15653 & -5.25620 & 1.48026 \\
H & 1.49152 & -6.05995 & -0.20146 \\
H & 2.99042 & -4.42711 & -1.32718 \\
H & 2.85327 & -2.05212 & -0.80612 \\
O & -0.98668 & -0.10942 & 1.72615 \\
O & 0.30252 & -0.65631 & 2.16070 \\
H & 2.30437 & 0.51910 & 2.06047 \\
H & 2.98271 & -0.96939 & 1.92770 \\
\end{array}$ & & & & & & & & & & \\
\hline $\begin{array}{llll}\text { S } & -1.52438 & -1.10262 & -0.93183 \\
\mathrm{C} & -0.83624 & 0.12856 & 0.21349 \\
\mathrm{C} & -1.59891 & 1.43913 & 0.13583 \\
\mathrm{~N} & -2.89675 & 1.42710 & -0.03100 \\
\mathrm{C} & -5.13549 & 0.55468 & -0.15304 \\
\mathrm{C} & -5.99943 & -0.49101 & -0.43145 \\
\mathrm{C} & -5.49074 & -1.72915 & -0.83856 \\
\mathrm{C} & -4.12146 & -1.92863 & -0.95913 \\
\mathrm{C} & -3.23921 & -0.88154 & -0.67961 \\
\mathrm{C} & -3.75679 & 0.35534 & -0.28408 \\
\mathrm{C} & -0.97074 & 2.74791 & 0.33229 \\
\mathrm{C} & -0.12462 & 2.98493 & 1.42945 \\
\mathrm{C} & 0.40296 & 4.25472 & 1.62541 \\
\mathrm{C} & 0.11159 & 5.28470 & 0.72964 \\
\mathrm{C} & -0.72286 & 5.05044 & -0.36199 \\
\mathrm{C} & -1.27375 & 3.78934 & -0.55978 \\
\mathrm{H} & -5.51577 & 1.52028 & 0.16855 \\
\mathrm{H} & -7.06944 & -0.34565 & -0.33048 \\
\mathrm{H} & -6.16857 & -2.54836 & -1.05546 \\
\mathrm{H} & -3.72932 & -2.89311 & -1.26601 \\
\mathrm{H} & 0.08325 & 2.19623 & 2.14664 \\
\mathrm{H} & 1.04007 & 4.44146 & 2.48393 \\
\mathrm{H} & 0.53702 & 6.27156 & 0.88387 \\
\mathrm{H} & -0.94247 & 5.84750 & -1.06492 \\
\mathrm{H} & -1.90138 & 3.60173 & -1.42663 \\
\mathrm{~S} & 1.35319 & 0.08557 & -1.66302 \\
\mathrm{C} & 0.66564 & 0.22783 & -0.01594 \\
\end{array}$ & $\begin{array}{c}- \\
- \\
2133.92271\end{array}$ & $\begin{array}{c}- \\
- \\
- \\
2133.46534\end{array}$ & $\begin{array}{c}- \\
- \\
- \\
2133.54978\end{array}$ & $\begin{array}{c}- \\
- \\
2133.95448\end{array}$ & $\begin{array}{c}- \\
- \\
- \\
2133.58155\end{array}$ & $\begin{array}{l}- \\
2135.17638\end{array}$ & $\begin{array}{c}- \\
- \\
\\
\\
\\
\\
\end{array}$ & $\begin{array}{l} \\
\\
\\
\end{array}$ & $\begin{array}{c}-2 \\
- \\
- \\
2135.20033\end{array}$ & 11.4 \\
\hline
\end{tabular}




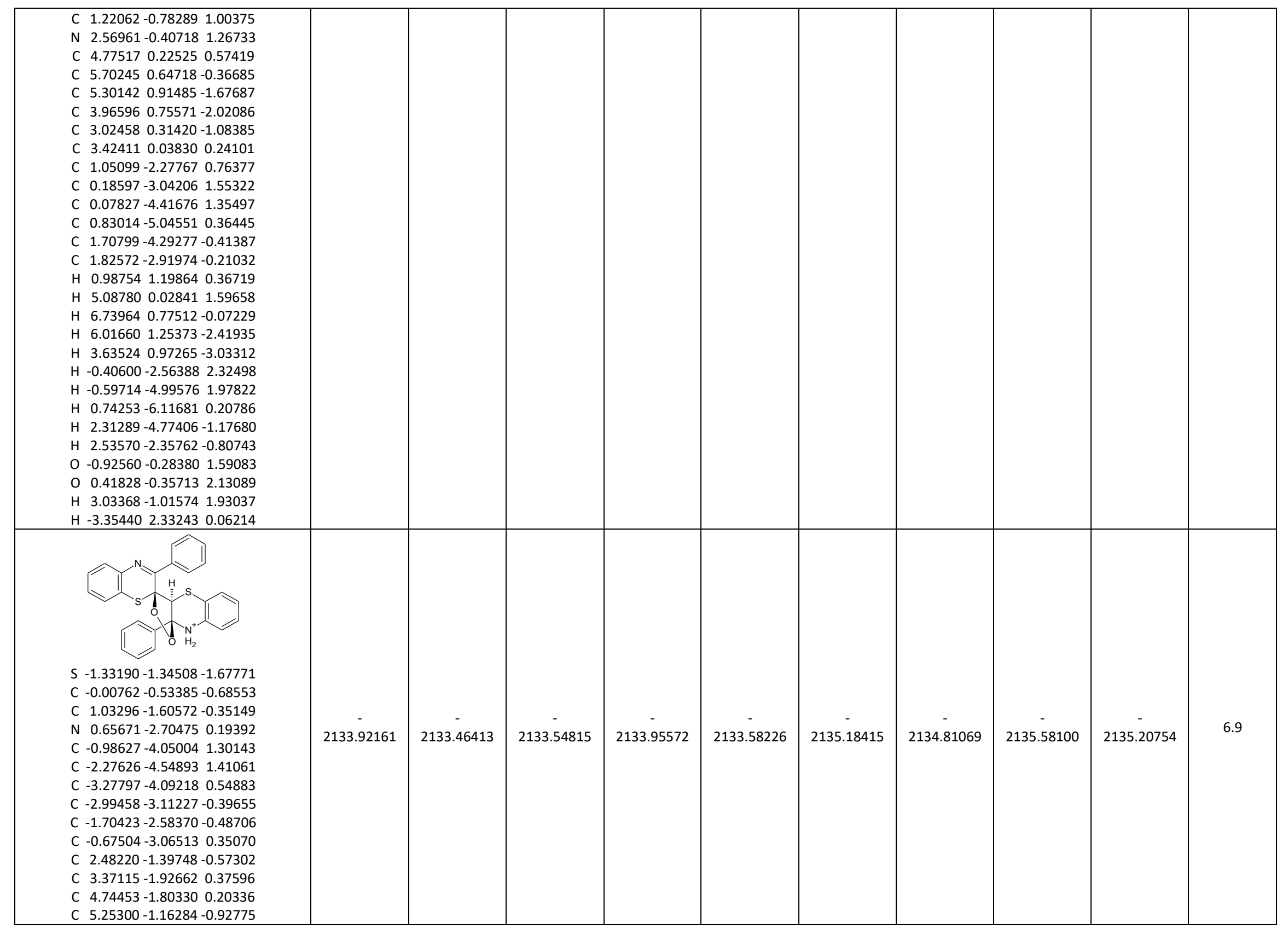




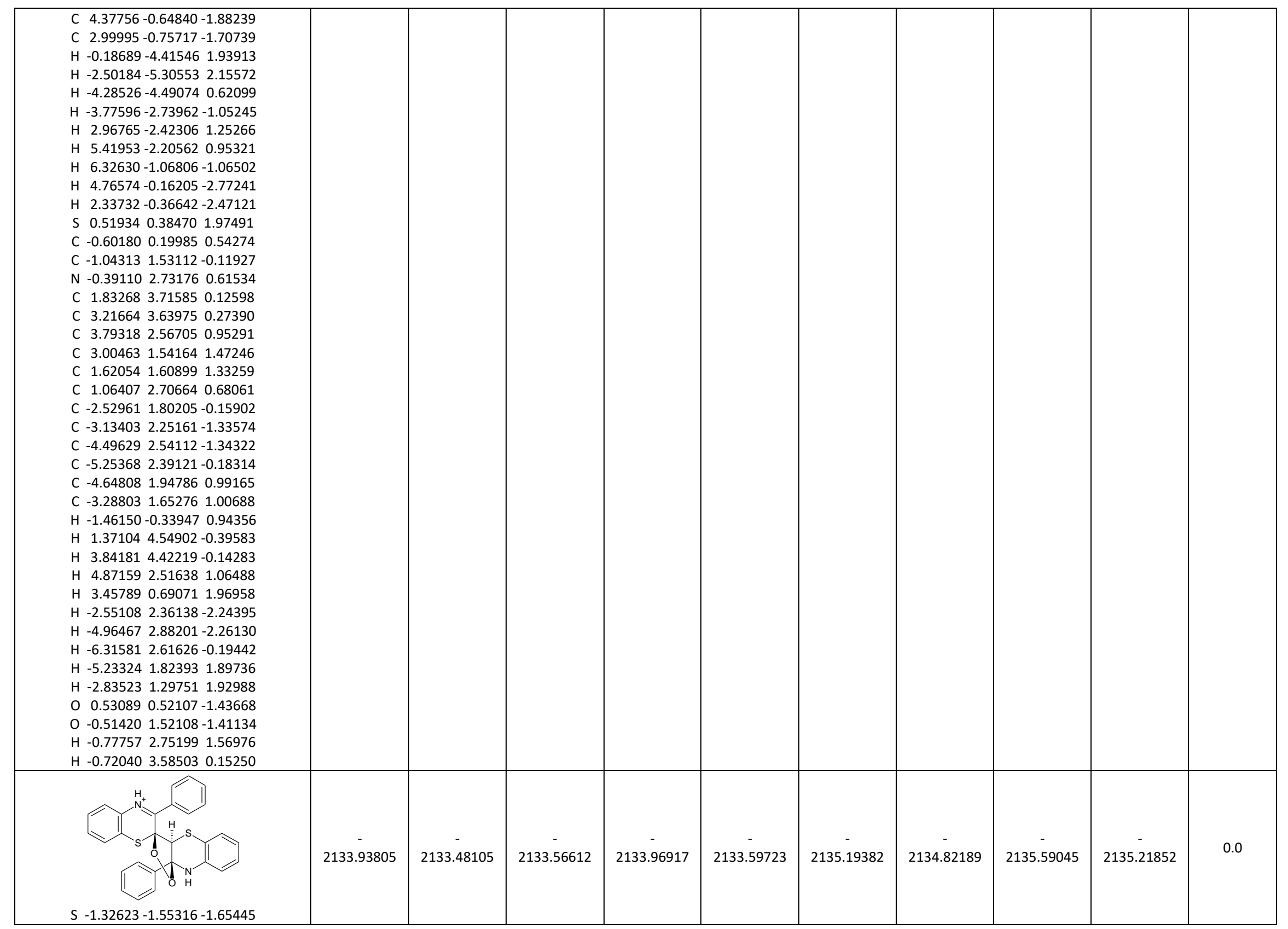

S151 
C $-0.06110-0.55241-0.74866$

C $1.04243-1.50940-0.34802$

N $0.66831-2.580260 .30684$

C - $0.87093-3.940631 .58093$

C - $2.13842-4.481911 .73740$

C $-3.16490-4.125530 .85908$

C $-2.93614-3.21619-0.16810$

C $-1.66817-2.65494-0.33330$

C $-0.64009-3.032980 .54472$

C $2.46415-1.30921-0.60569$

C $3.39718-1.721590 .36580$

C $4.75676-1.597570 .12266$

C $5.20462-1.07743-1.09250$

C $4.28678-0.67110-2.05992$

C $2.92191-0.77150-1.82139$

H - $-0.06292-4.212592 .25357$

H -2.32682 -5.182282 .54391$

H $-4.15607-4.549890 .98254$

H -3.73941-2.93024-0.83935

H $3.06549-2.084461 .33499$

H $5.46721-1.895730 .88679$

H $6.26959-0.98433-1.28208$

H $4.63323-0.27377-3.00850$

H $2.22076-0.47041-2.58949$

$\begin{array}{llllllll}\text { S } & 0.40007 & 0.42927 & 1.88928\end{array}$

$\begin{array}{llll}\text { C }-0.66667 & 0.26266 & 0.42725\end{array}$

C $-1.02542 \quad 1.61702-0.27072$

$\begin{array}{lllll}\text { N }-0.31537 & 2.73468 & 0.26746\end{array}$

C 1.924853 .703390 .12870

C 3.263913 .670500 .51048

C 3.757352 .613991 .27598

C 2.902381 .583641 .66335

C $1.55717 \quad 1.62853 \quad 1.30384$

C $1.05623 \quad 2.68747 \quad 0.53541$

C -2.51749 $1.91857-0.26205$

C $-3.35027 \quad 1.54475-1.32049$

C - 4.71643 1.81596-1.26229

C -5.26295 $2.44860-0.14747$

C $-4.43463 \quad 2.81647 \quad 0.91188$

C - $-3.06715 \quad 2.557850 .85439$

H $-1.57153-0.217070 .80304$

H $1.54448 \quad 4.52392-0.47439$

H 3.926634 .471920 .19761

H 4.803042 .585901 .56588

H 3.273440 .753432 .25698

H -2.93593 $1.04684-2.18946$

H $-5.353301 .53012-2.09437$

H -6.32837 $2.65538-0.10476$

H -4.85062 3.309331 .78571

H -2.42169 2.850241 .67670

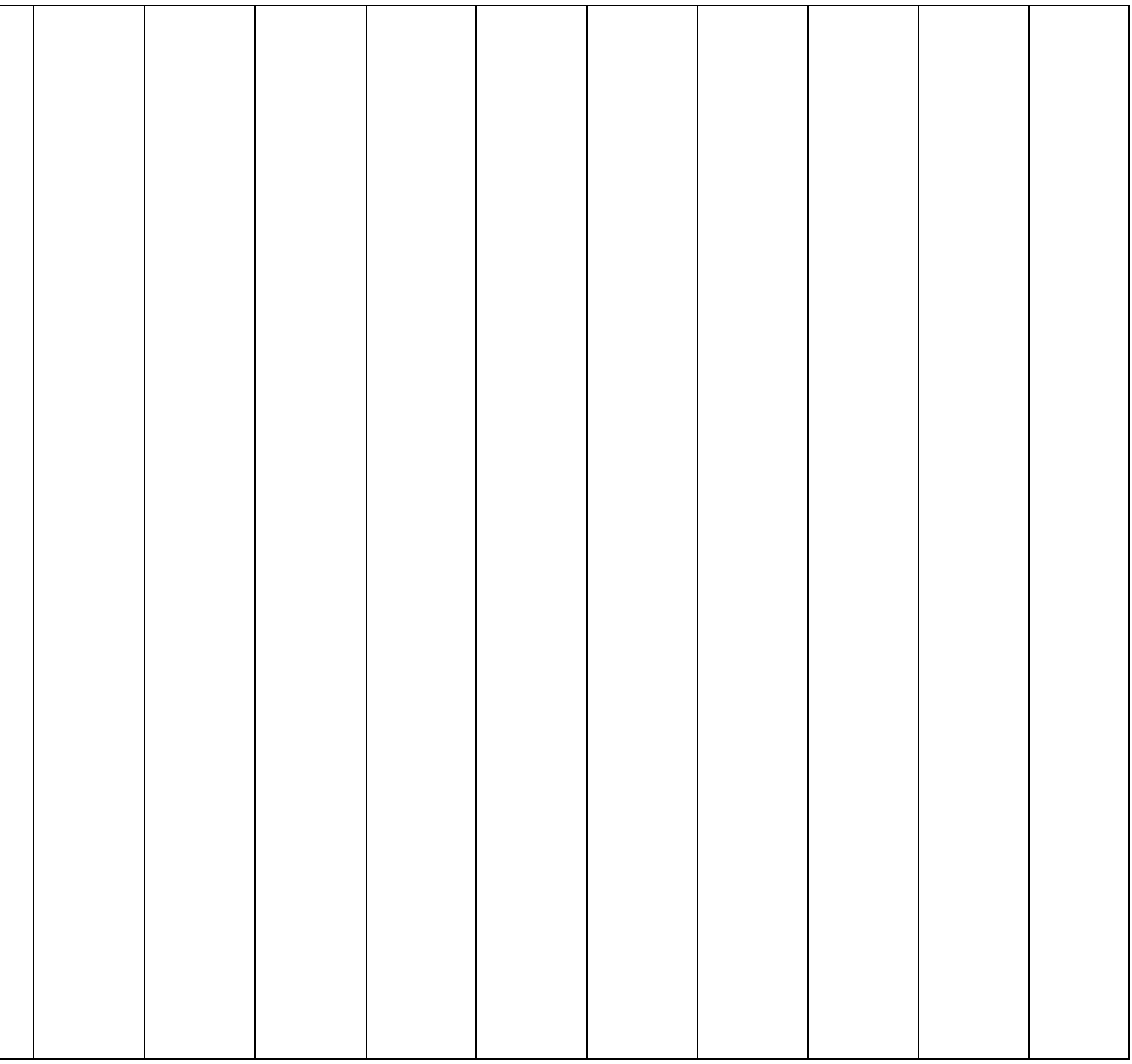




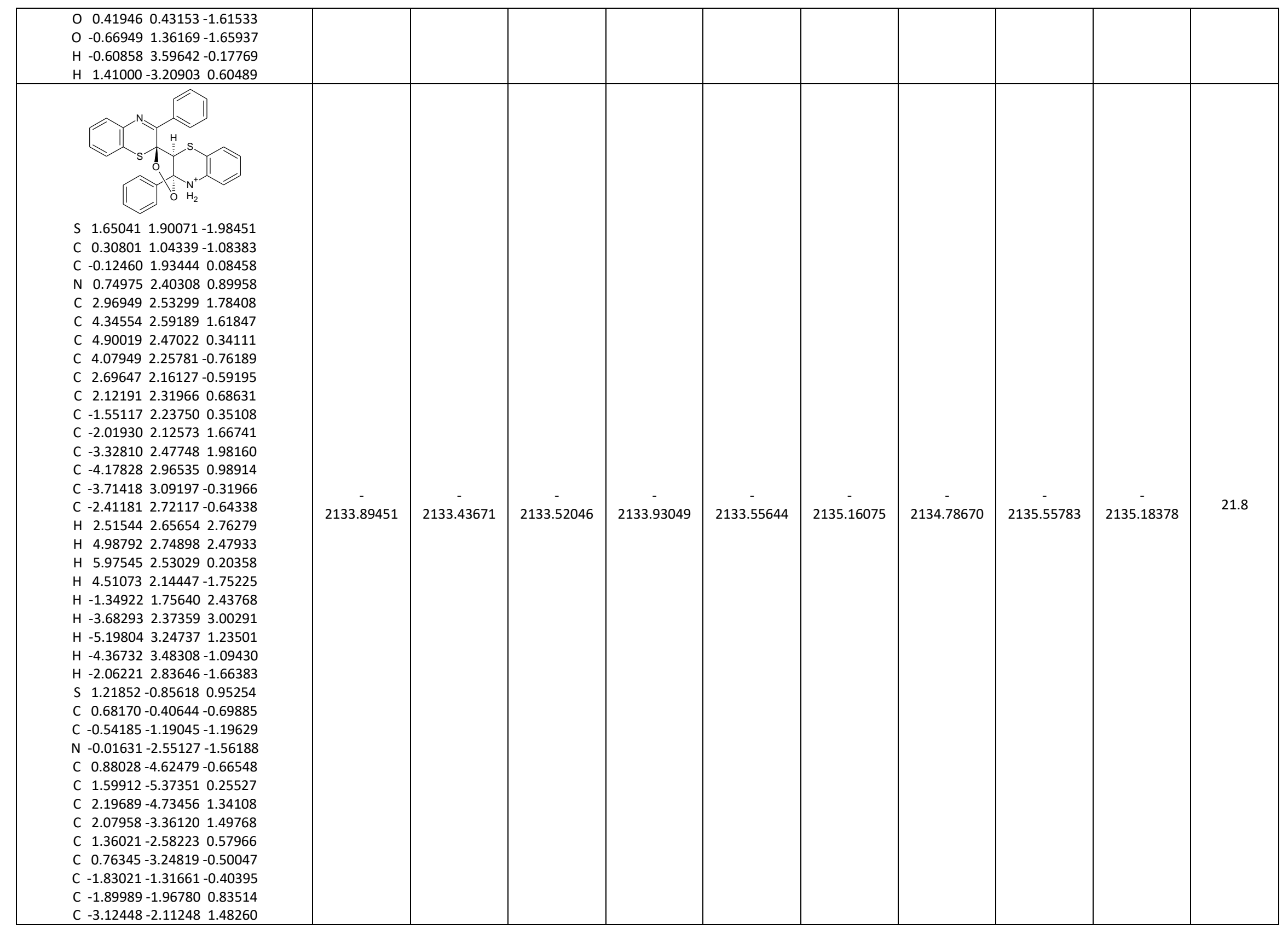




\begin{tabular}{|c|c|c|c|c|c|c|c|c|c|c|}
\hline 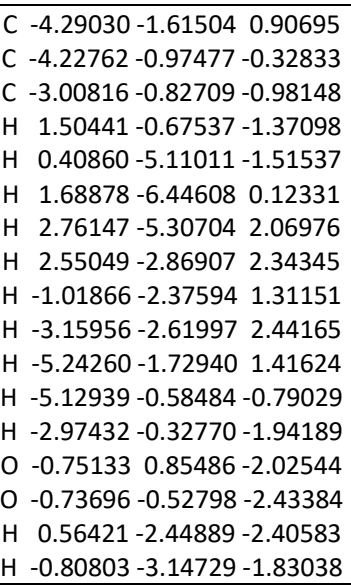 & & & & & & & & & & \\
\hline $\begin{array}{llll}\text { S } & -0.57343 & -2.34359 & -2.00941 \\
C & 0.21125 & -0.94321 & -1.11223 \\
C & 1.05533 & -1.54371 & -0.00407 \\
\mathrm{~N} & 0.46236 & -2.39408 & 0.79622 \\
\mathrm{C} & -1.40327 & -3.59323 & 1.75372 \\
\mathrm{C} & -2.59756 & -4.27793 & 1.58616 \\
\mathrm{C} & -3.18080 & -4.36524 & 0.31962 \\
\mathrm{C} & -2.58371 & -3.75979 & -0.78125 \\
\mathrm{C} & -1.38826 & -3.05614 & -0.62580 \\
\mathrm{C} & -0.80351 & -2.98833 & 0.64763 \\
\mathrm{C} & 2.45240 & -1.21131 & 0.25975 \\
\mathrm{C} & 2.86005 & -1.00063 & 1.58949 \\
\mathrm{C} & 4.19911 & -0.77012 & 1.87461 \\
\mathrm{C} & 5.14220 & -0.77426 & 0.84685 \\
\mathrm{C} & 4.74187 & -0.99100 & -0.47156 \\
\mathrm{C} & 3.40199 & -1.19268 & -0.77543 \\
\mathrm{H} & -0.93689 & -3.51734 & 2.73159 \\
\mathrm{H} & -3.07387 & -4.74452 & 2.44165 \\
\mathrm{H} & -4.11554 & -4.90092 & 0.18899 \\
\mathrm{H} & -3.04782 & -3.82058 & -1.76035 \\
\mathrm{H} & 2.12636 & -0.96479 & 2.39067 \\
\mathrm{H} & 4.50449 & -0.58192 & 2.89884 \\
\mathrm{H} & 6.19019 & -0.60314 & 1.07335 \\
\mathrm{H} & 5.47656 & -1.00136 & -1.27033 \\
\mathrm{H} & 3.10261 & -1.36994 & -1.80130\end{array}$ & $\begin{array}{c}2 \\
- \\
2133.91502\end{array}$ & $\begin{array}{c}- \\
- \\
- \\
2133.45798\end{array}$ & $\begin{array}{c}- \\
- \\
2133.54199\end{array}$ & $\begin{array}{c}- \\
- \\
2133.94649\end{array}$ & $\begin{array}{c}-2 \\
\\
\\
\\
- \\
2133.57346\end{array}$ & $\begin{array}{l}- \\
\\
\\
\\
\\
\\
\\
\\
\\
\end{array}$ & $\begin{array}{c}- \\
- \\
2134.80075\end{array}$ & $\begin{array}{c}2 \\
- \\
2135.57067\end{array}$ & $\begin{array}{c}- \\
- \\
- \\
2135.19764\end{array}$ & 13.1 \\
\hline
\end{tabular}




\begin{tabular}{|c|c|c|c|c|c|c|c|c|c|c|}
\hline $\begin{array}{llll}\text { S } & -1.49421 & 0.23434 & 0.98136 \\
\text { C } & -0.79439 & 0.14007 & -0.66962 \\
\text { C } & -0.10571 & 1.43942 & -1.12912 \\
\text { N } & -1.14532 & 2.38544 & -1.37668 \\
\text { C } & -3.08003 & 3.61883 & -0.67907 \\
\text { C } & -4.17148 & 3.84336 & 0.14640 \\
\text { C } & -4.45472 & 2.96197 & 1.19129 \\
\text { C } & -3.63207 & 1.86169 & 1.38924 \\
\text { C } & -2.51713 & 1.63526 & 0.57343 \\
\text { C } & -2.22570 & 2.52162 & -0.48496 \\
\text { C } & 1.05628 & 2.03137 & -0.33860 \\
\text { C } & 0.83174 & 2.61864 & 0.91410 \\
\text { C } & 1.87274 & 3.23739 & 1.60220 \\
\text { C } & 3.14794 & 3.29751 & 1.04327 \\
\text { C } & 3.37243 & 2.73772 & -0.21238 \\
\text { C } & 2.33584 & 2.10768 & -0.89772 \\
\text { H } & -1.63878 & 0.02712 & -1.35721 \\
\text { H } & -2.86757 & 4.30127 & -1.49825 \\
\text { H } & -4.80618 & 4.70587 & -0.03277 \\
\text { H } & -5.31081 & 3.12302 & 1.83841 \\
\text { H } & -3.84539 & 1.15982 & 2.19114 \\
\text { H } & -0.15779 & 2.61519 & 1.35642 \\
\text { H } & 1.67944 & 3.68400 & 2.57327 \\
\text { H } & 3.95743 & 3.78599 & 1.57812 \\
\text { H } & 4.35863 & 2.78747 & -0.66484 \\
\text { H } & 2.52517 & 1.67929 & -1.87507 \\
\text { O } & 1.03046 & -0.27208 & -2.04911 \\
\text { O } & 0.35110 & 0.94988 & -2.40962 \\
\text { H } & -0.79917 & 3.27691 & -1.71044 \\
\text { H } & 1.03229 & -2.76517 & 1.55418\end{array}$ & & & & & & & & & & \\
\hline Double-bond dimers & \multirow[b]{2}{*}{$\begin{array}{c}- \\
1982.50999\end{array}$} & \multirow[b]{2}{*}{$\begin{array}{c}- \\
1982.08785\end{array}$} & \multirow[b]{2}{*}{$\begin{array}{c}- \\
1982.17029\end{array}$} & \multirow[b]{2}{*}{$\begin{array}{c}- \\
1982.53709\end{array}$} & \multirow[b]{2}{*}{$\begin{array}{c}- \\
1982.19739\end{array}$} & \multirow[b]{2}{*}{$\begin{array}{c}- \\
1983.66106\end{array}$} & \multirow[b]{2}{*}{$\begin{array}{c}- \\
1983.32136\end{array}$} & \multirow[b]{2}{*}{$\begin{array}{c}- \\
1984.01083\end{array}$} & \multirow[b]{2}{*}{$\begin{array}{c}- \\
1983.67113\end{array}$} & \multirow[b]{2}{*}{0.0} \\
\hline $\begin{array}{lrrr}\text { S } & -1.30306 & 2.41219 & -0.91938 \\
C & -0.57651 & 0.84003 & -0.48839 \\
\text { C } & -1.36681 & -0.31109 & -0.82649 \\
\text { N } & -2.68895 & -0.23152 & -0.77386 \\
C & -4.75303 & 0.59928 & 0.17334 \\
C & -5.51996 & 1.66300 & 0.63387 \\
C & -4.98551 & 2.95141 & 0.65872 \\
C & -3.68195 & 3.18359 & 0.22605 \\
C & -2.90963 & 2.12318 & -0.24393 \\
C & -3.44996 & 0.83347 & -0.26628 \\
C & -0.80041 & -1.55367 & -1.35954\end{array}$ & & & & & & & & & & \\
\hline
\end{tabular}




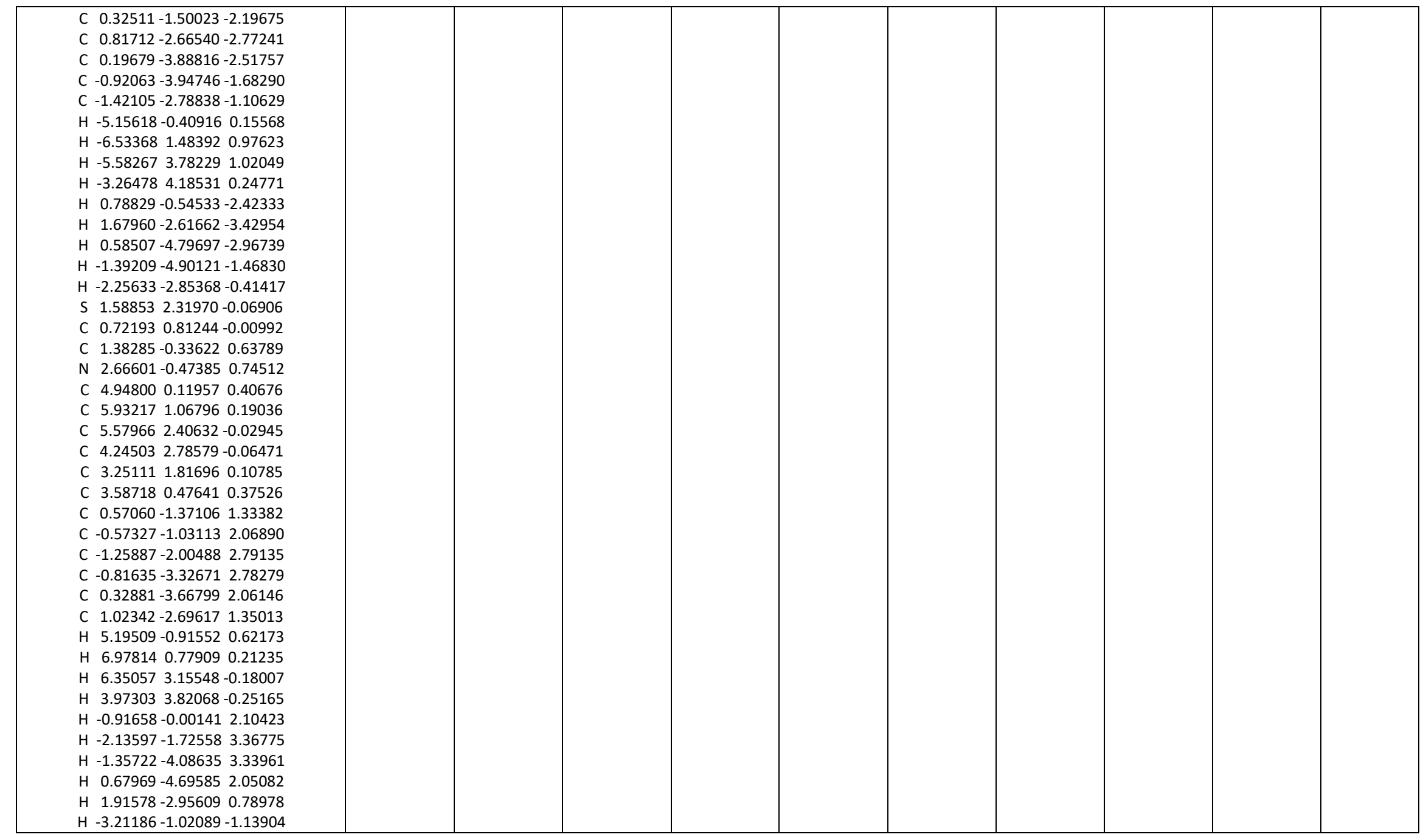


Table S6. Diprotonated forms in methanol. For each chemical species, values are reported for the most stable conformer / geometric isomer identified. $G_{\text {RRHO,calc }}[M 062 X / 6-311++G(2 d, 2 p) / S M D]=G_{\text {el }}[M 062 X / 6-311++G(2 d, 2 p) / S M D / / P B E 0 / 6-31+G(d, p) / P C M]-G_{\text {el }}[P B E 0 / 6-31+G(d, p) / P C M]+$

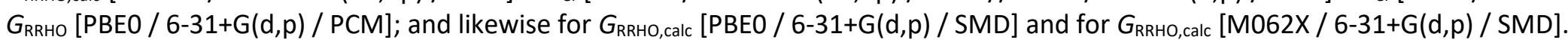

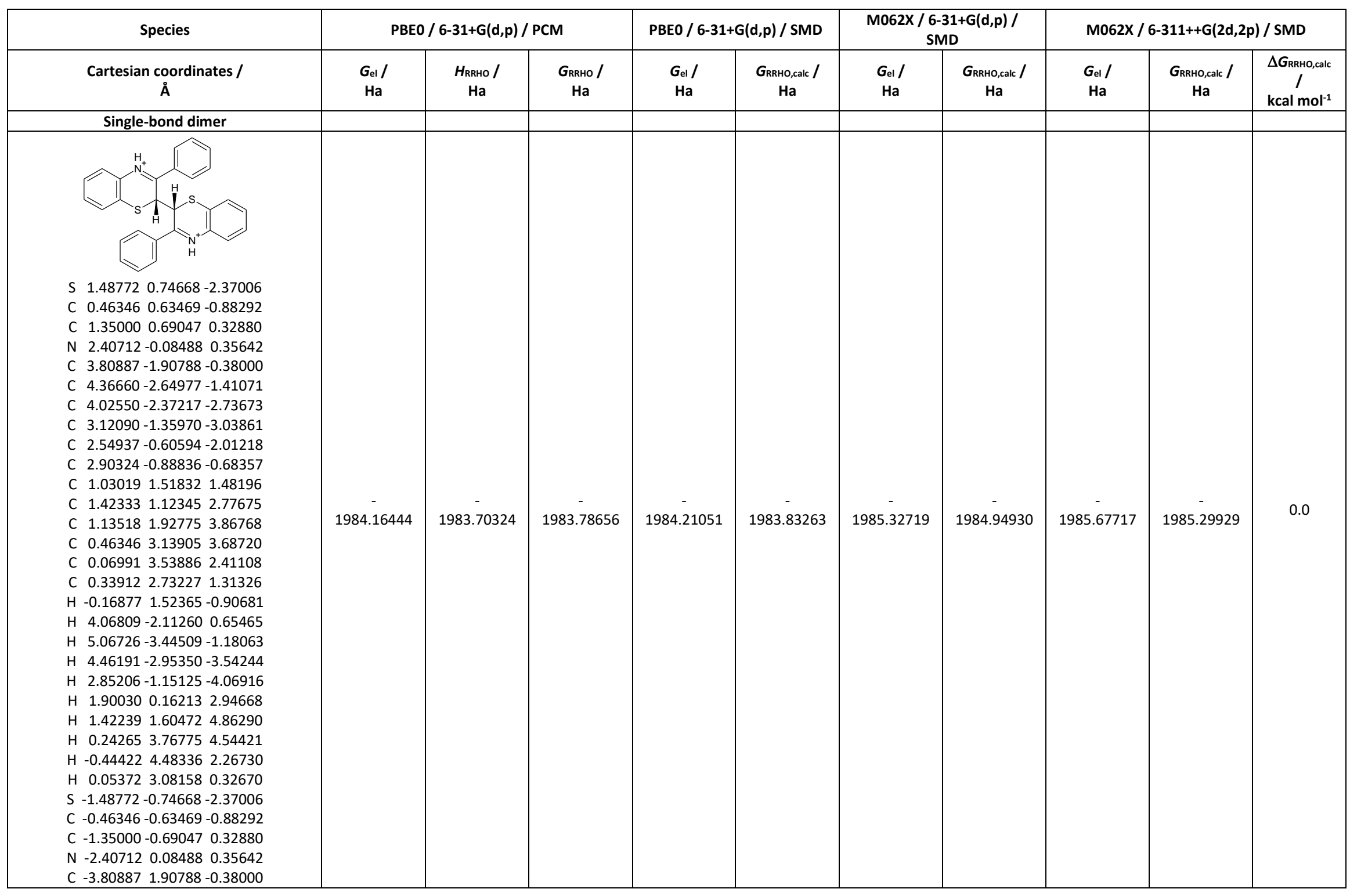




\begin{tabular}{|c|c|c|c|c|c|c|c|c|c|c|}
\hline $\begin{array}{llll}\text { C } & -4.36660 & 2.64977 & -1.41071 \\
\text { C } & -4.02550 & 2.37217 & -2.73673 \\
\text { C } & -3.12090 & 1.35970 & -3.03861 \\
\text { C } & -2.54937 & 0.60594 & -2.01218 \\
\text { C } & -2.90324 & 0.88836 & -0.68357 \\
\text { C } & -1.03019 & -1.51832 & 1.48196 \\
\text { C } & -1.42333 & -1.12345 & 2.77675 \\
\text { C } & -1.13518 & -1.92775 & 3.86768 \\
\text { C } & -0.46346 & -3.13905 & 3.68720 \\
\text { C } & -0.06991 & -3.53886 & 2.41108 \\
\text { C } & -0.33912 & -2.73227 & 1.31326 \\
\text { H } & 0.16877 & -1.52365 & -0.90681 \\
\text { H } & -4.06809 & 2.11260 & 0.65465 \\
\text { H } & -5.06726 & 3.44509 & -1.18063 \\
\text { H } & -4.46191 & 2.95350 & -3.54244 \\
\text { H } & -2.85206 & 1.15125 & -4.06916 \\
\text { H } & -1.90030 & -0.16213 & 2.94668 \\
\text { H } & -1.42239 & -1.60472 & 4.86290 \\
\text { H } & -0.24265 & -3.76775 & 4.54421 \\
\text { H } & 0.44422 & -4.48336 & 2.26730 \\
\text { H } & -0.05372 & -3.08158 & 0.32670 \\
\text { H } & 2.98748 & -0.04771 & 1.19062 \\
\text { H } & -2.98748 & 0.04771 & 1.19062 \\
\end{array}$ & & & & & & & & & & \\
\hline 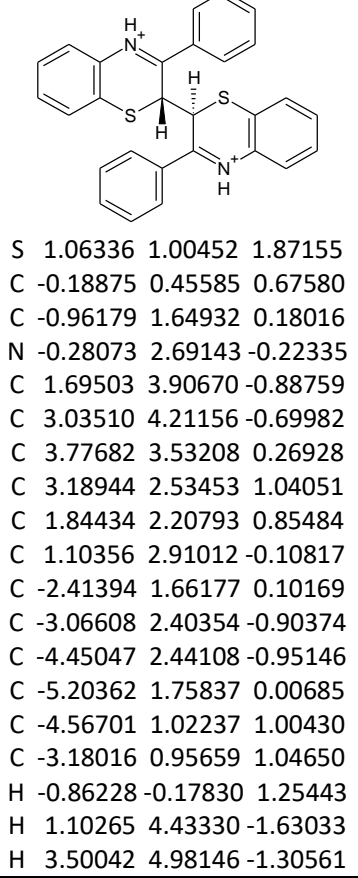 & $\begin{array}{c}- \\
1984.16481\end{array}$ & $\begin{array}{c}- \\
- \\
- \\
1983.70346\end{array}$ & $\begin{array}{c}- \\
- \\
- \\
1983.78586\end{array}$ & - & $\begin{array}{c}-2.00 \\
- \\
- \\
1983.82950\end{array}$ & $\begin{array}{c}2.0 . \\
- \\
1985.32702\end{array}$ & $\begin{array}{c}- \\
1984.94806\end{array}$ & $\begin{array}{c}- \\
1985.67748\end{array}$ & $\begin{array}{c}- \\
1985.29853\end{array}$ & 0.5 \\
\hline
\end{tabular}




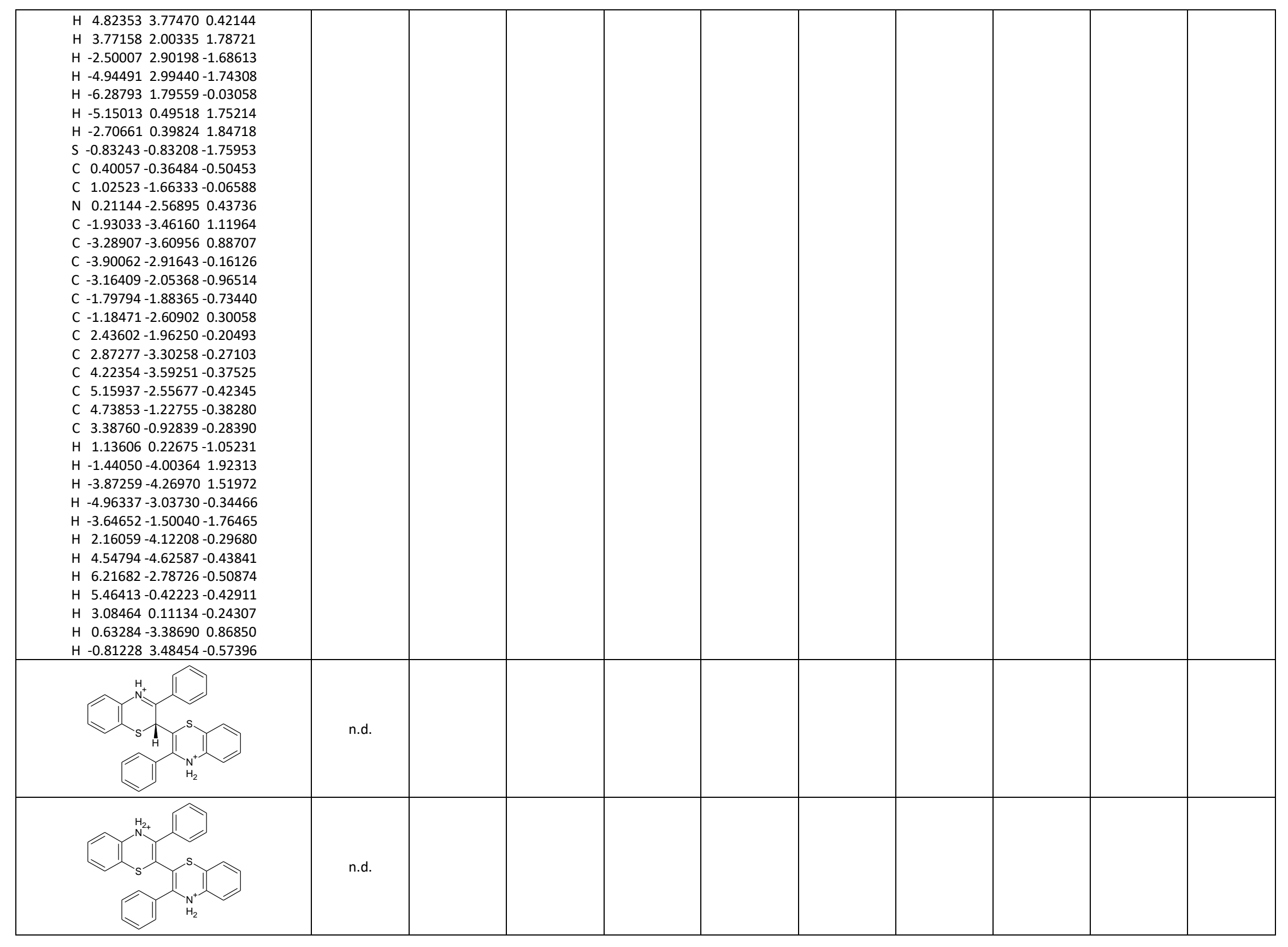




\begin{tabular}{|c|c|c|c|c|c|c|c|c|c|c|}
\hline Single-bond dimer, radical & & & & & & & & & & \\
\hline $\begin{array}{llll} & \end{array}$ & $\begin{array}{c}- \\
- \\
1983.54274\end{array}$ & $\begin{array}{c}- \\
- \\
1983.09441\end{array}$ & $\begin{array}{c}- \\
1983.17911\end{array}$ & $\begin{array}{c}- \\
- \\
1983.58334\end{array}$ & $\stackrel{-}{-}$ & $\begin{array}{c}- \\
- \\
- \\
1984.69628\end{array}$ & $\begin{array}{c}- \\
- \\
- \\
1984.33264\end{array}$ & $\begin{array}{c}- \\
- \\
1985.04719\end{array}$ & 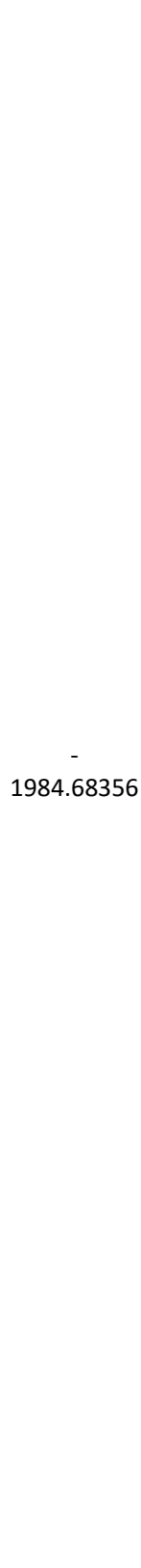 & 0.0 \\
\hline
\end{tabular}




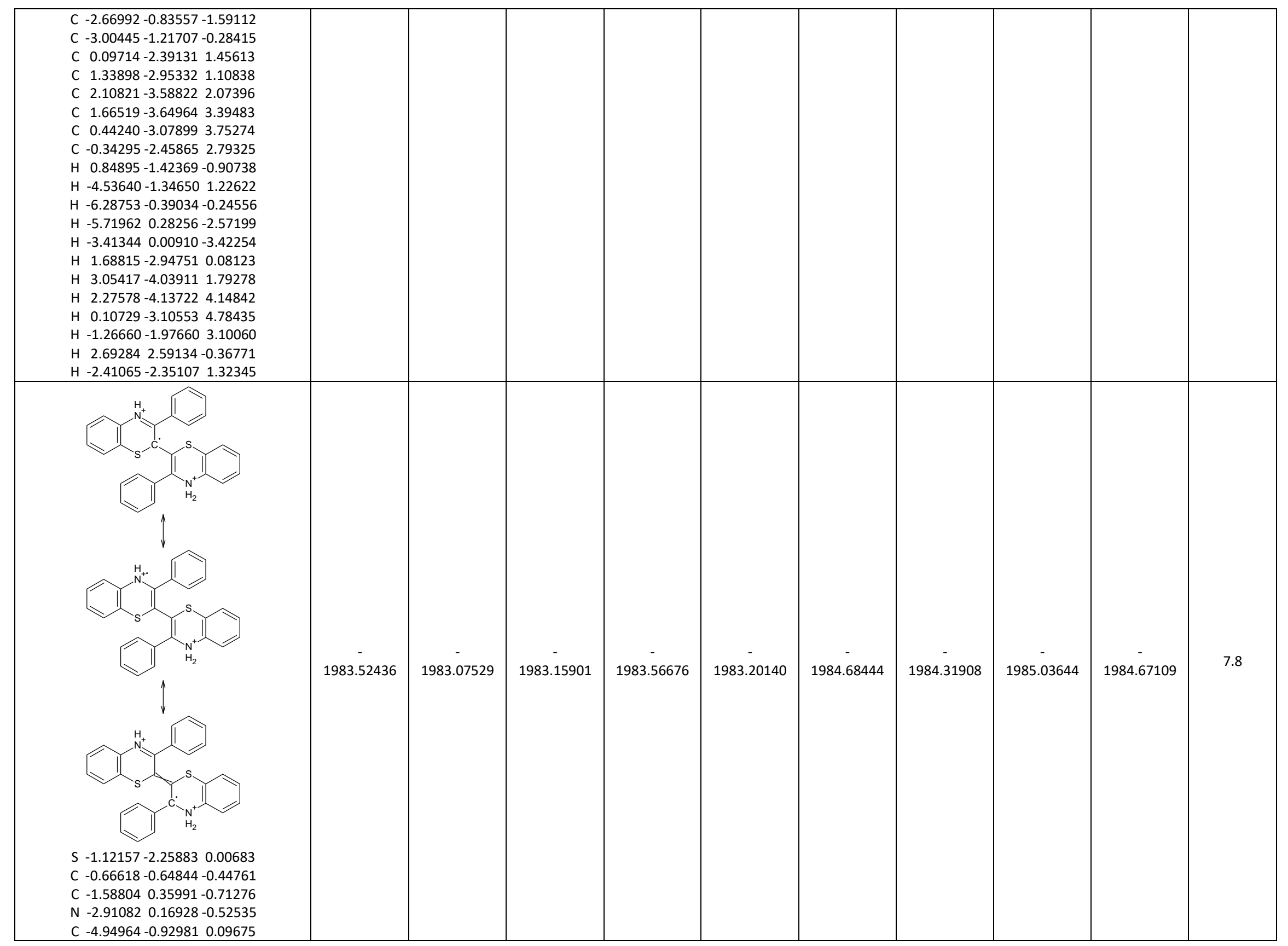




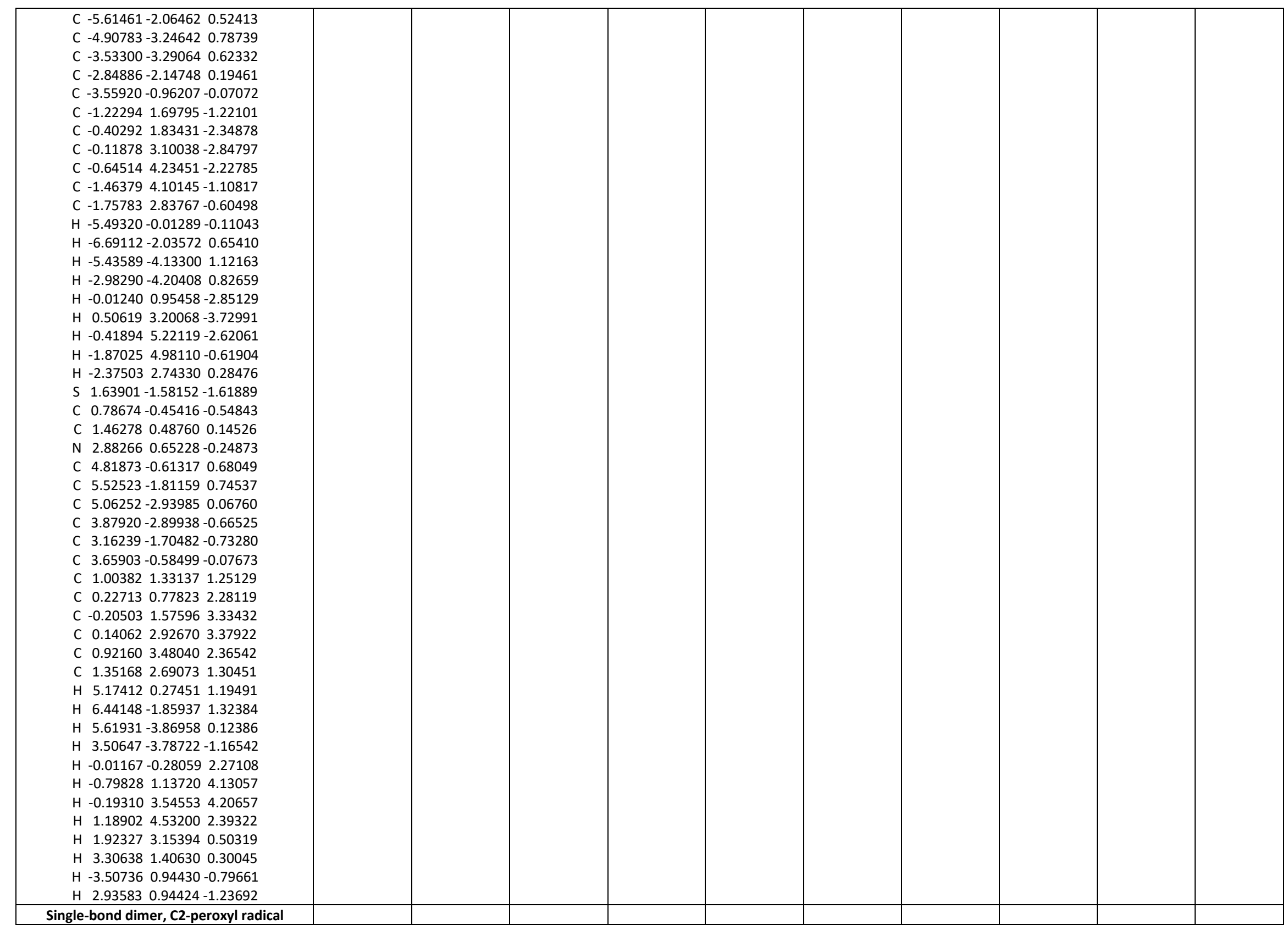




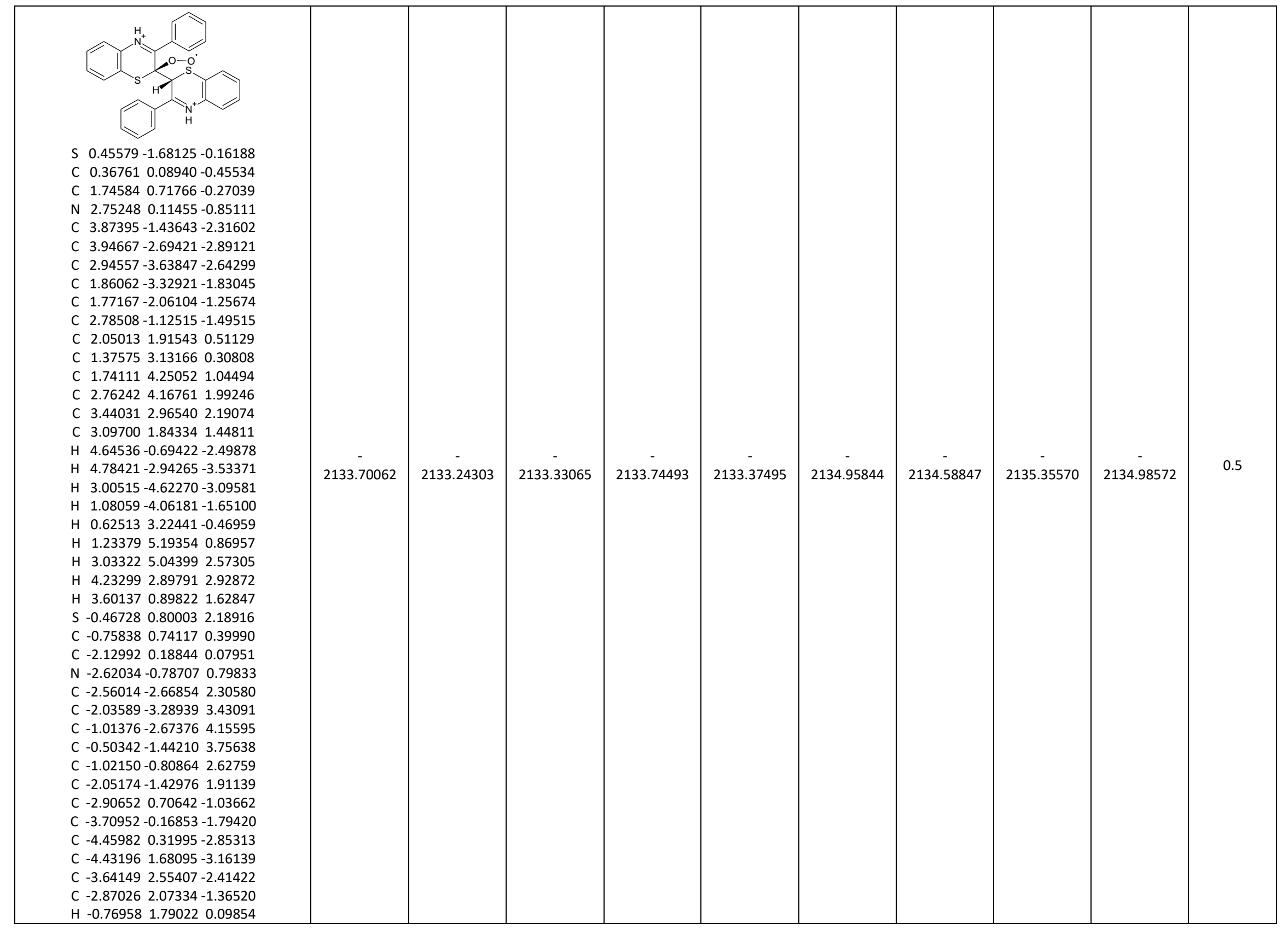




\begin{tabular}{|c|c|c|c|c|c|c|c|c|c|c|}
\hline $\begin{array}{rrrr}H & -3.35003 & -3.13926 & 1.72799 \\
\text { H } & -2.42360 & -4.25366 & 3.74099 \\
\text { H } & -0.60379 & -3.15893 & 5.03578 \\
\text { H } & 0.29614 & -0.96955 & 4.31798 \\
\text { H } & -3.70190 & -1.23714 & -1.59922 \\
\text { H } & -5.05630 & -0.36384 & -3.44789 \\
\text { H } & -5.02271 & 2.06019 & -3.98934 \\
\text { H } & -3.62699 & 3.61367 & -2.64674 \\
\text { H } & -2.28993 & 2.77712 & -0.77775 \\
\text { O } & -0.00522 & 0.24622 & -1.87979 \\
\text { O } & 0.18813 & 1.47431 & -2.28232 \\
\text { H } & -3.55631 & -1.10337 & 0.55082 \\
\text { H } & 3.64222 & 0.60977 & -0.81207 \\
\end{array}$ & & & & & & & & & & \\
\hline $\begin{array}{llll}\text { S } & 1.15006 & -1.03758 & 1.58687 \\
\mathrm{C} & -0.17830 & -0.43824 & 0.48298 \\
\mathrm{C} & -0.90855 & -1.68987 & 0.01151 \\
\mathrm{~N} & -0.17646 & -2.58714 & -0.61309 \\
\mathrm{C} & 1.85220 & -3.48894 & -1.57613 \\
\mathrm{C} & 3.22584 & -3.66125 & -1.50729 \\
\mathrm{C} & 3.96396 & -3.02764 & -0.50348 \\
\mathrm{C} & 3.33881 & -2.20239 & 0.42459 \\
\mathrm{C} & 1.95721 & -2.01131 & 0.36217 \\
\mathrm{C} & 1.22155 & -2.67234 & -0.63331 \\
\mathrm{C} & -2.30366 & -1.99042 & 0.27594 \\
\mathrm{C} & -2.64673 & -3.32708 & 0.57183 \\
\mathrm{C} & -3.96984 & -3.67322 & 0.79822 \\
\mathrm{C} & -4.966699 & -2.69914 & 0.72633 \\
\mathrm{C} & -4.63624 & -1.37355 & 0.44107 \\
\mathrm{C} & -3.31467 & -1.01130 & 0.22856 \\
\mathrm{H} & 1.26591 & -3.98073 & -2.34621 \\
\mathrm{H} & 3.72379 & -4.29274 & -2.23498 \\
\mathrm{H} & 5.03890 & -3.16623 & -0.45134 \\
\mathrm{H} & 3.91794 & -1.69713 & 1.19067 \\
\mathrm{H} & -1.87254 & -4.08098 & 0.68172 \\
\mathrm{H} & -4.22202 & -4.69905 & 1.04552 \\
\mathrm{H} & -6.00258 & -2.97173 & 0.90327 \\
\mathrm{H} & -5.41259 & -0.61776 & 0.38359 \\
\mathrm{H} & -3.07931 & 0.01886 & -0.00466 \\
\mathrm{~S} & -0.94338 & 0.78495 & -1.97046 \\
\mathrm{C} & 0.31300 & 0.45154 & -0.70297 \\
\mathrm{C} & 0.95747 & 1.72839 & -0.22880 \\
\mathrm{~N} & 0.20502 & 2.77745 & -0.01804\end{array}$ & $\begin{array}{c} \\
- \\
\\
\\
\\
\end{array}$ & - & $\begin{array}{l}- \\
- \\
2133.33259\end{array}$ & $\begin{array}{c}- \\
- \\
- \\
2133.74530\end{array}$ & $\begin{array}{l}- \\
2133.37564\end{array}$ & $\begin{array}{l}- \\
2134.95889\end{array}$ & $\begin{array}{l}- \\
\\
\\
\\
\end{array}$ & $\begin{array}{c}- \\
\\
\\
\\
- \\
2135.35612\end{array}$ & $\begin{array}{c}- \\
2134.98646\end{array}$ & 0.0 \\
\hline
\end{tabular}




\begin{tabular}{|c|c|c|c|c|c|c|c|c|c|c|}
\hline $\begin{array}{lrrr}\text { C } & -1.83349 & 4.01865 & 0.30656 \\
\text { C } & -3.16574 & 4.24490 & -0.00846 \\
\text { C } & -3.82178 & 3.40494 & -0.90982 \\
\text { C } & -3.15669 & 2.32734 & -1.48808 \\
\text { C } & -1.82079 & 2.08221 & -1.16970 \\
\text { C } & -1.16549 & 2.94133 & -0.27767 \\
\text { C } & 2.38587 & 1.80980 & 0.03389 \\
\text { C } & 2.86823 & 2.61527 & 1.08435 \\
\text { C } & 4.23225 & 2.72087 & 1.30651 \\
\text { C } & 5.13128 & 2.04447 & 0.47902 \\
\text { C } & 4.66184 & 1.24803 & -0.56443 \\
\text { C } & 3.29681 & 1.11455 & -0.78115 \\
\text { H } & 1.07035 & -0.13423 & -1.23063 \\
\text { H } & -1.31021 & 4.66658 & 1.00358 \\
\text { H } & -3.69074 & 5.07759 & 0.44665 \\
\text { H } & -4.86171 & 3.58459 & -1.16237 \\
\text { H } & -3.67235 & 1.67558 & -2.18664 \\
\text { H } & 2.18171 & 3.11009 & 1.76530 \\
\text { H } & 4.59574 & 3.32250 & 2.13298 \\
\text { H } & 6.19903 & 2.13483 & 0.65264 \\
\text { H } & 5.35948 & 0.72920 & -1.21352 \\
\text { H } & 2.95410 & 0.51755 & -1.61974 \\
\text { O } & -1.08145 & 0.36590 & 1.27026 \\
\text { O } & -0.76845 & 0.42661 & 2.53624 \\
\text { H } & 0.68189 & 3.61917 & 0.29991 \\
\text { H } & -0.67826 & -3.35981 & -1.04569 \\
\end{array}$ & & & & & & & & & & \\
\hline \multicolumn{11}{|l|}{ Single-bond dimer, C3-peroxyl radical } \\
\hline $\begin{array}{llll}\text { S } & -0.96547 & 1.68992 & 1.48735 \\
\text { C } & -0.94642 & 0.16157 & 0.59295 \\
\text { C } & -2.23631 & -0.16623 & -0.02936 \\
\text { N } & -2.88591 & 0.79658 & -0.64214 \\
\text { C } & -3.10648 & 2.94767 & -1.72020 \\
\text { C } & -2.81181 & 4.30274 & -1.75451 \\
\text { C } & -1.94440 & 4.85568 & -0.80949 \\
C & -1.35703 & 4.05789 & 0.16715\end{array}$ & $\begin{array}{c}- \\
2133.68733\end{array}$ & $\begin{array}{c}- \\
2133.22926\end{array}$ & $\begin{array}{c}- \\
2133.31674\end{array}$ & $\begin{array}{c}- \\
2133.73224\end{array}$ & $\begin{array}{c}- \\
2133.36165\end{array}$ & $\begin{array}{c}- \\
2134.94842\end{array}$ & $\begin{array}{c}- \\
2134.57783\end{array}$ & $\begin{array}{c}- \\
2135.34718\end{array}$ & $\begin{array}{c}- \\
2134.97659\end{array}$ & 0.0 \\
\hline
\end{tabular}




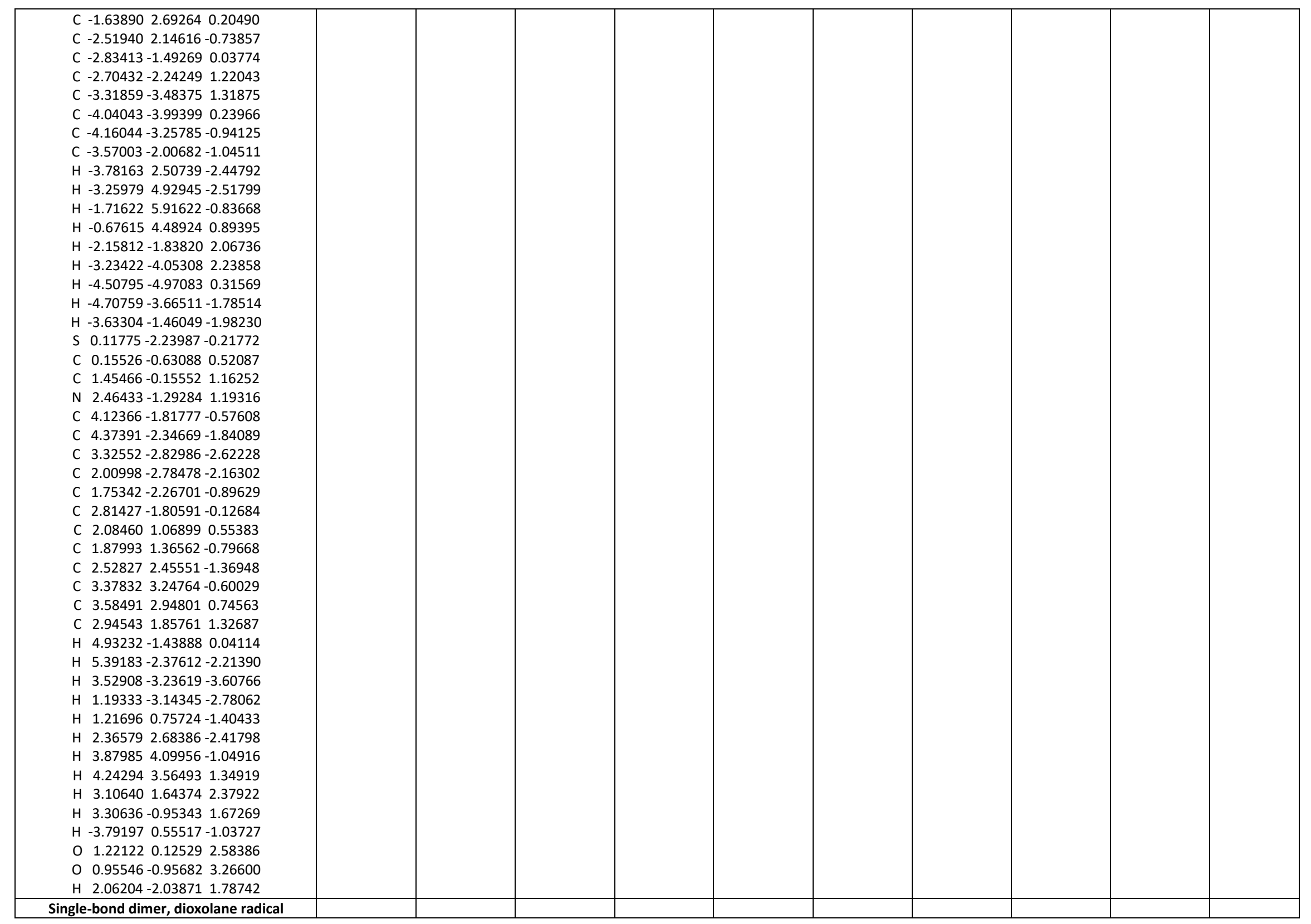




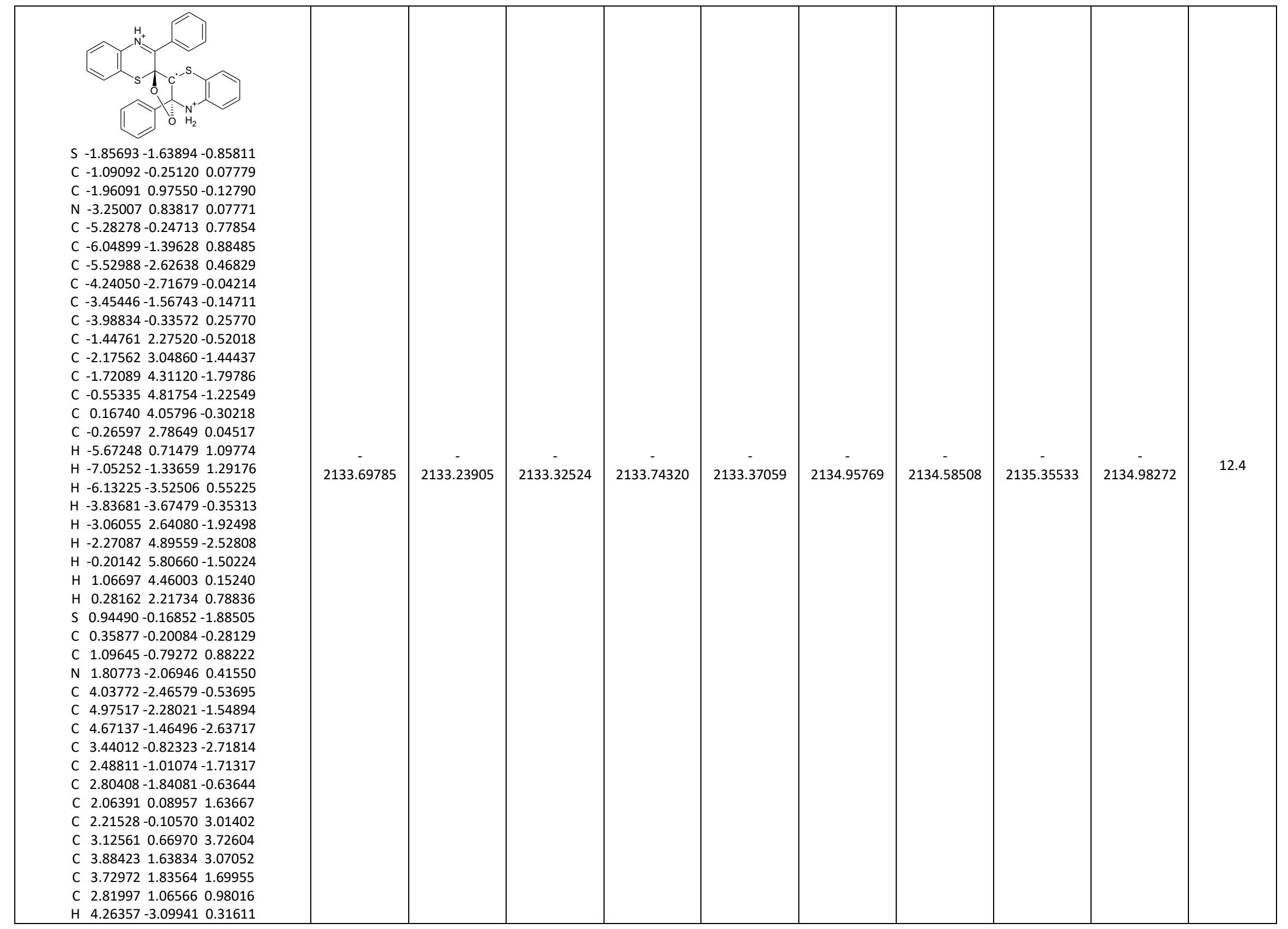




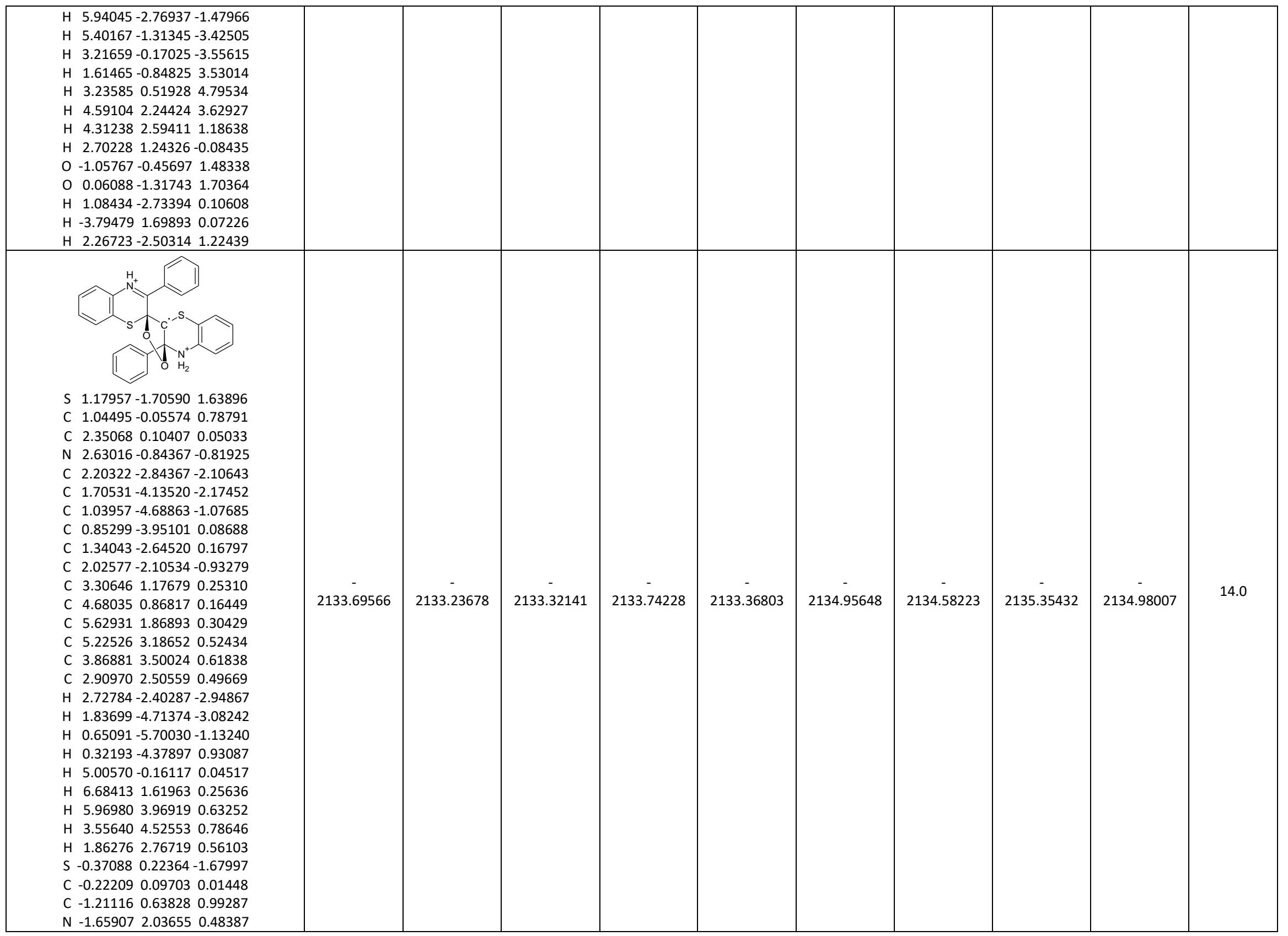




\begin{tabular}{|c|c|c|c|c|c|c|c|c|c|c|}
\hline $\begin{array}{llll}\text { C } & -3.52099 & 2.71456 & -0.97264 \\
\text { C } & -4.17970 & 2.67429 & -2.19837 \\
\text { C } & -3.67756 & 1.88805 & -3.23294 \\
\text { C } & -2.52404 & 1.13337 & -3.04941 \\
\text { C } & -1.85015 & 1.17523 & -1.82647 \\
\text { C } & -2.36006 & 1.97582 & -0.80196 \\
\text { C } & -2.44527 & -0.16262 & 1.32893 \\
\text { C } & -2.77320 & -1.30784 & 0.60311 \\
\text { C } & -3.91378 & -2.03360 & 0.93886 \\
\text { C } & -4.71757 & -1.62348 & 1.99987 \\
\text { C } & -4.38147 & -0.48523 & 2.73290 \\
\text { C } & -3.24762 & 0.24841 & 2.40035 \\
\text { H } & -3.90650 & 3.32391 & -0.16057 \\
\text { H } & -5.08575 & 3.25376 & -2.33712 \\
\text { H } & -4.19088 & 1.84994 & -4.18797 \\
\text { H } & -2.14562 & 0.50772 & -3.85165 \\
\text { H } & -2.14502 & -1.64132 & -0.21697 \\
\text { H } & -4.16959 & -2.92181 & 0.36991 \\
\text { H } & -5.60496 & -2.19242 & 2.26000 \\
\text { H } & -4.99909 & -0.16858 & 3.56727 \\
\text { H } & -2.98760 & 1.12105 & 2.99499 \\
\text { O } & 0.92075 & 0.92537 & 1.79518 \\
\text { O } & -0.46883 & 0.82141 & 2.17559 \\
\text { H } & -2.27697 & 2.45587 & 1.18723 \\
\text { H } & 3.43221 & -0.67849 & -1.42374 \\
\text { H } & -0.83761 & 2.65083 & 0.42708\end{array}$ & & & & & & & & & & \\
\hline $\begin{array}{lllll} & & & & \end{array}$ & $\begin{array}{c}- \\
2133.72169\end{array}$ & $\begin{array}{c}- \\
2133.26445\end{array}$ & $\begin{array}{c}- \\
2133.35065\end{array}$ & $\begin{array}{c}- \\
2133.76427\end{array}$ & $\begin{array}{c}- \\
2133.39322\end{array}$ & $\begin{array}{c}- \\
2134.97674\end{array}$ & $\begin{array}{c}- \\
2134.60570\end{array}$ & $\begin{array}{c}- \\
2135.37329\end{array}$ & $\begin{array}{c}- \\
2135.00224\end{array}$ & 0.1 \\
\hline
\end{tabular}




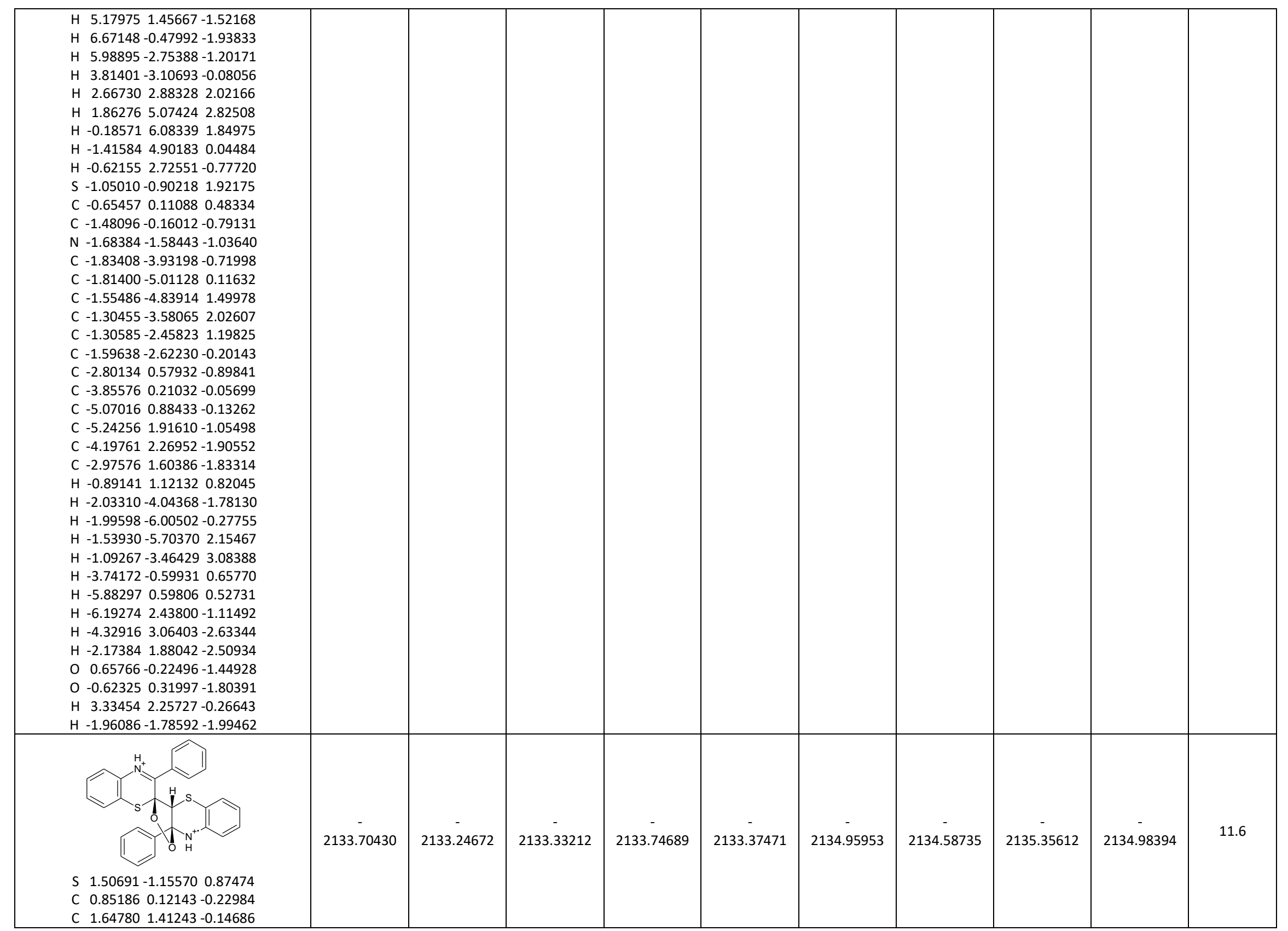


N 2.940971 .367320 .04008

$\begin{array}{llll}\text { C } 5.15991 & 0.45333 & 0.19715\end{array}$

C $5.99812-0.613030 .47485$

C $5.45922-1.848520 .84900$

$\begin{array}{llll}C & 4.08422-2.02614 & 0.93773\end{array}$

C $3.22965-0.957370 .65957$

$\begin{array}{llll}\text { C } 3.77536 & 0.27741 & 0.29713\end{array}$

C $1.044872 .73265-0.34175$

C $0.228812 .99669-1.45587$

C $-0.280494 .27499-1.64313$

$0.000515 .28671-0.72338$

C 0.805965 .025720 .38373

C 1.337603 .755450 .57508

H $5.564221 .41610-0.10214$

H $7.07234-0.485940 .39807$

H $6.11750-2.683581 .06520$

H 3.66917-2.98859 1.21926

H $0.036282 .22603-2.19635$

H $-0.891784 .48374-2.51515$

H - $-0.40918 \quad 6.28090-0.87235$

H 1.018465 .809281 .10369

H 1.942353 .546541 .45303

S - $1.338860 .18152 \quad 1.66287$

C -0.645630 .263060 .01109$

C $-1.22363-0.74717-0.98925$

$\mathrm{N}-2.59983-0.35321-1.20361$

C $-4.790010 .30291-0.57915$

C -5.689170 .691140 .37263$

C $-5.267620 .90741 \quad 1.70845$

$\begin{array}{llll}C & -3.93981 & 0.74841 & 2.06647\end{array}$

C -2.984500 .371541 .11888$

C $-3.417720 .11116-0.24064$

C $-1.12650-2.24778-0.75815$

C $-0.30973-3.02247-1.58888$

C $-0.22535-4.39865-1.39862$

C $-0.95569-5.01406-0.38431$

C $-1.78742-4.248390 .43145$

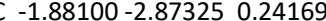

H $-0.937991 .23990-0.38439$

H $-5.100710 .12558-1.60394$

H -6.730890 .831920 .10651$

H $-5.98891 \quad 1.216532 .45726$

$\begin{array}{llll}-3.62612 & 0.93648 & 3.08817\end{array}$

H $0.26416-2.55291-2.37893$

H $0.41387-4.98889-2.04796$

H $-0.88772-6.08772-0.23695$

H $-2.37426-4.720431 .21316$

H $-2.55492-2.308800 .87648$

O $0.88778-0.26298-1.62771$

O $-0.47028-0.34043-2.12946$

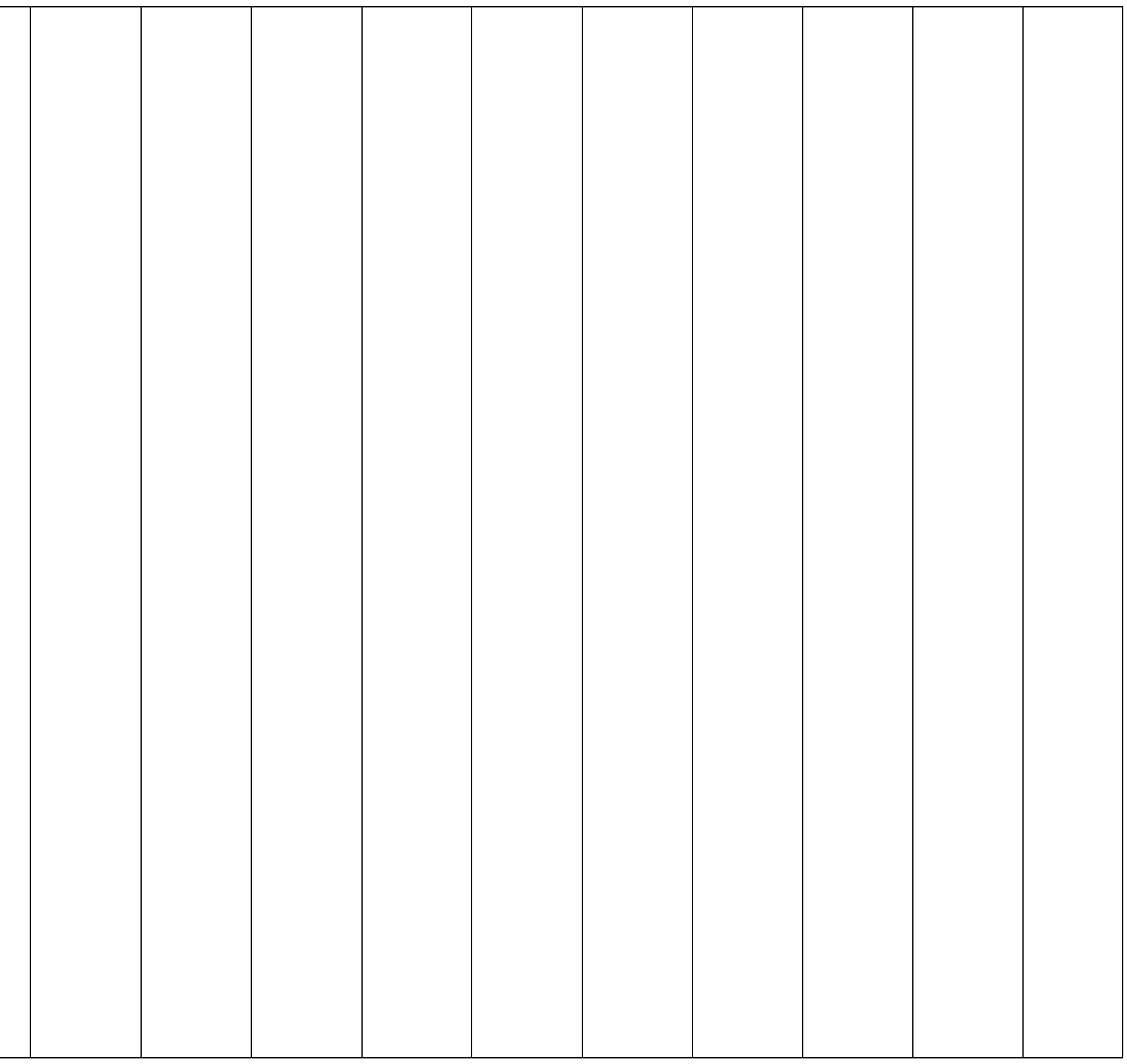




\begin{tabular}{|c|c|c|c|c|c|c|c|c|c|c|}
\hline $\begin{array}{lrrr}H & 3.41938 & 2.26391 & -0.03932 \\
H & -3.02952 & -0.65846 & -2.07341 \\
\end{array}$ & & & & & & & & & & \\
\hline $\begin{array}{llll} & \end{array}$ & $\begin{array}{c}- \\
- \\
2133.72133\end{array}$ & $\begin{array}{c}- \\
2133.26362\end{array}$ & $\begin{array}{c}- \\
2133.35026\end{array}$ & $\begin{array}{c}- \\
2133.76319\end{array}$ & - & $\begin{array}{c}- \\
2134.97707\end{array}$ & 2134.60601 & $\begin{array}{c}- \\
- \\
2135.37348\end{array}$ & $\begin{array}{c}- \\
2135.00242\end{array}$ & 0.0 \\
\hline
\end{tabular}




\begin{tabular}{|c|c|c|c|c|c|c|c|c|c|c|}
\hline $\begin{array}{lrrr}\text { C } & -3.08379 & 2.72089 & 0.41077 \\
\text { H } & -1.72559 & -0.53609 & 0.43473 \\
\text { H } & 1.25430 & 4.45980 & -0.52036 \\
\text { H } & 3.11911 & 4.88806 & 1.04437 \\
\text { H } & 3.59415 & 3.28485 & 2.89200 \\
\text { H } & 2.22877 & 1.25038 & 3.17695 \\
\text { H } & -3.39719 & 0.11247 & -1.76171 \\
\text { H } & -5.78188 & 0.67366 & -1.47285 \\
\text { H } & -6.45584 & 2.53650 & 0.02657 \\
\text { H } & -4.72049 & 3.84015 & 1.23482 \\
\text { H } & -2.33415 & 3.29668 & 0.94481 \\
\text { O } & 0.18489 & 0.19576 & -1.92087 \\
\text { O } & -1.05616 & 0.90992 & -1.94246 \\
\text { H } & 2.09878 & -2.78576 & 0.64730 \\
\text { H } & -0.56074 & 3.19599 & -1.03514 \\
\end{array}$ & & & & & & & & & & \\
\hline $\begin{array}{llll}\text { S } & -0.45036 & -2.37990 & -2.00733 \\
\mathrm{C} & 0.25081 & -0.94049 & -1.11667 \\
\mathrm{C} & 1.14314 & -1.48233 & -0.01352 \\
\mathrm{~N} & 0.59765 & -2.35461 & 0.79755 \\
\mathrm{C} & -1.21173 & -3.62502 & 1.77546 \\
\mathrm{C} & -2.37568 & -4.36139 & 1.61736 \\
\mathrm{C} & -2.95949 & -4.48486 & 0.35391 \\
\mathrm{C} & -2.39382 & -3.86483 & -0.75523 \\
\mathrm{C} & -1.22876 & -3.11015 & -0.61005 \\
\mathrm{C} & -0.64196 & -3.00441 & 0.66119 \\
\mathrm{C} & 2.52575 & -1.08368 & 0.22276 \\
\mathrm{C} & 2.95153 & -0.87429 & 1.54809 \\
\mathrm{C} & 4.28257 & -0.58100 & 1.80875 \\
\mathrm{C} & 5.20224 & -0.52063 & 0.76151 \\
\mathrm{C} & 4.78575 & -0.73751 & -0.55183 \\
\mathrm{C} & 3.45206 & -1.00249 & -0.83176 \\
\mathrm{H} & -0.74460 & -3.52321 & 2.75061 \\
\mathrm{H} & -2.82818 & -4.84114 & 2.47835 \\
\mathrm{H} & -3.87075 & -5.06132 & 0.23211 \\
\mathrm{H} & -2.85836 & -3.95517 & -1.73175 \\
\mathrm{H} & 2.23575 & -0.88692 & 2.36587 \\
\mathrm{H} & 4.60018 & -0.39384 & 2.82933 \\
\mathrm{H} & 6.24478 & -0.30001 & 0.96925 \\
\mathrm{H} & 5.50309 & -0.70036 & -1.36528 \\
\mathrm{H} & 3.14520 & -1.18592 & -1.85430 \\
\mathrm{~S} & -1.62784 & 0.10772 & 0.92016 \\
\mathrm{C} & -0.81781 & 0.08726 & -0.68465\end{array}$ & . & $\begin{array}{l}- \\
- \\
2133.23934\end{array}$ & $\begin{array}{c}- \\
2133.32534\end{array}$ & $\begin{array}{l}- \\
- \\
\\
\\
\\
\\
\end{array}$ & $\begin{array}{c}- \\
2133.36739\end{array}$ & $\begin{array}{c}- \\
2134.95665\end{array}$ & $\begin{array}{c}- \\
\\
\\
\\
\\
\\
\\
\end{array}$ & $\begin{array}{l}- \\
2135.35326\end{array}$ & $\begin{array}{c}- \\
2134.98180\end{array}$ & 12.9 \\
\hline
\end{tabular}




\begin{tabular}{|c|c|c|c|c|c|c|c|c|c|c|}
\hline $\begin{array}{llll}\text { C } & -0.16032 & 1.41475 & -1.08320 \\
\text { N } & -1.24036 & 2.35605 & -1.28391 \\
\text { C } & -3.22943 & 3.52116 & -0.72515 \\
\text { C } & -4.31408 & 3.69827 & 0.08572 \\
\text { C } & -4.56791 & 2.79651 & 1.14974 \\
\text { C } & -3.73770 & 1.71269 & 1.37211 \\
\text { C } & -2.62635 & 1.48622 & 0.55390 \\
\text { C } & -2.33949 & 2.42824 & -0.51006 \\
\text { C } & 0.95418 & 2.05835 & -0.27025 \\
\text { C } & 0.72509 & 2.54633 & 1.02280 \\
\text { C } & 1.74289 & 3.19067 & 1.71850 \\
\text { C } & 2.99286 & 3.37150 & 1.12793 \\
\text { C } & 3.21762 & 2.90846 & -0.16590 \\
\text { C } & 2.20465 & 2.25835 & -0.86494 \\
\text { H } & -1.62319 & -0.04883 & -1.41563 \\
H & -3.02008 & 4.20779 & -1.53954 \\
\text { H } & -4.98390 & 4.53419 & -0.08304 \\
\text { H } & -5.43215 & 2.94680 & 1.78780 \\
\text { H } & -3.95551 & 1.01611 & 2.17539 \\
H & -0.24156 & 2.44614 & 1.50244 \\
H & 1.55204 & 3.56024 & 2.72119 \\
\text { H } & 3.78373 & 3.87976 & 1.67141 \\
H & 4.18371 & 3.05320 & -0.63946 \\
\text { H } & 2.38921 & 1.90969 & -1.87402 \\
\text { O } & 1.01972 & -0.20473 & -2.05802 \\
\text { O } & 0.29990 & 1.00433 & -2.37059 \\
\text { H } & 1.19190 & -2.69625 & 1.55106 \\
\text { H } & -1.03771 & 3.15276 & -1.88278\end{array}$ & & & & & & & & & & \\
\hline Single-bond dimer, dioxane radic & & & & & & & & & & \multirow[b]{2}{*}{6.3} \\
\hline $\begin{array}{llll}\text { S } & -1.50857 & -0.47385 & -1.86598 \\
\text { C } & -0.47297 & 0.07425 & -0.57228 \\
\text { C } & -1.09572 & 0.18613 & 0.80263 \\
\text { N } & -2.22601 & 1.09188 & 0.72661 \\
\text { C } & -4.40982 & 1.71288 & -0.00870 \\
\text { C } & -5.44210 & 1.53601 & -0.88777 \\
\text { C } & -5.30689 & 0.66123 & -1.99745 \\
\text { C } & -4.11827 & 0.00231 & -2.23531 \\
\text { C } & -3.03009 & 0.18588 & -1.37038 \\
\text { C } & -3.19237 & 1.00389 & -0.19778 \\
\text { C } & -1.46399 & -1.16423 & 1.40392 \\
\text { C } & -2.44343 & -1.22654 & 2.40084 \\
\text { C } & -2.73352 & -2.43949 & 3.01688\end{array}$ & $\begin{array}{c}- \\
2133.69369\end{array}$ & $\begin{array}{c}- \\
2133.23512\end{array}$ & $\begin{array}{c}- \\
2133.32059\end{array}$ & $\begin{array}{c}- \\
2133.73956\end{array}$ & $\begin{array}{c}- \\
2133.36646\end{array}$ & $\begin{array}{c}- \\
2134.95833\end{array}$ & $\begin{array}{c}- \\
2134.58523\end{array}$ & $\begin{array}{c}- \\
2135.35649\end{array}$ & $\begin{array}{c}- \\
2134.98339\end{array}$ & \\
\hline
\end{tabular}




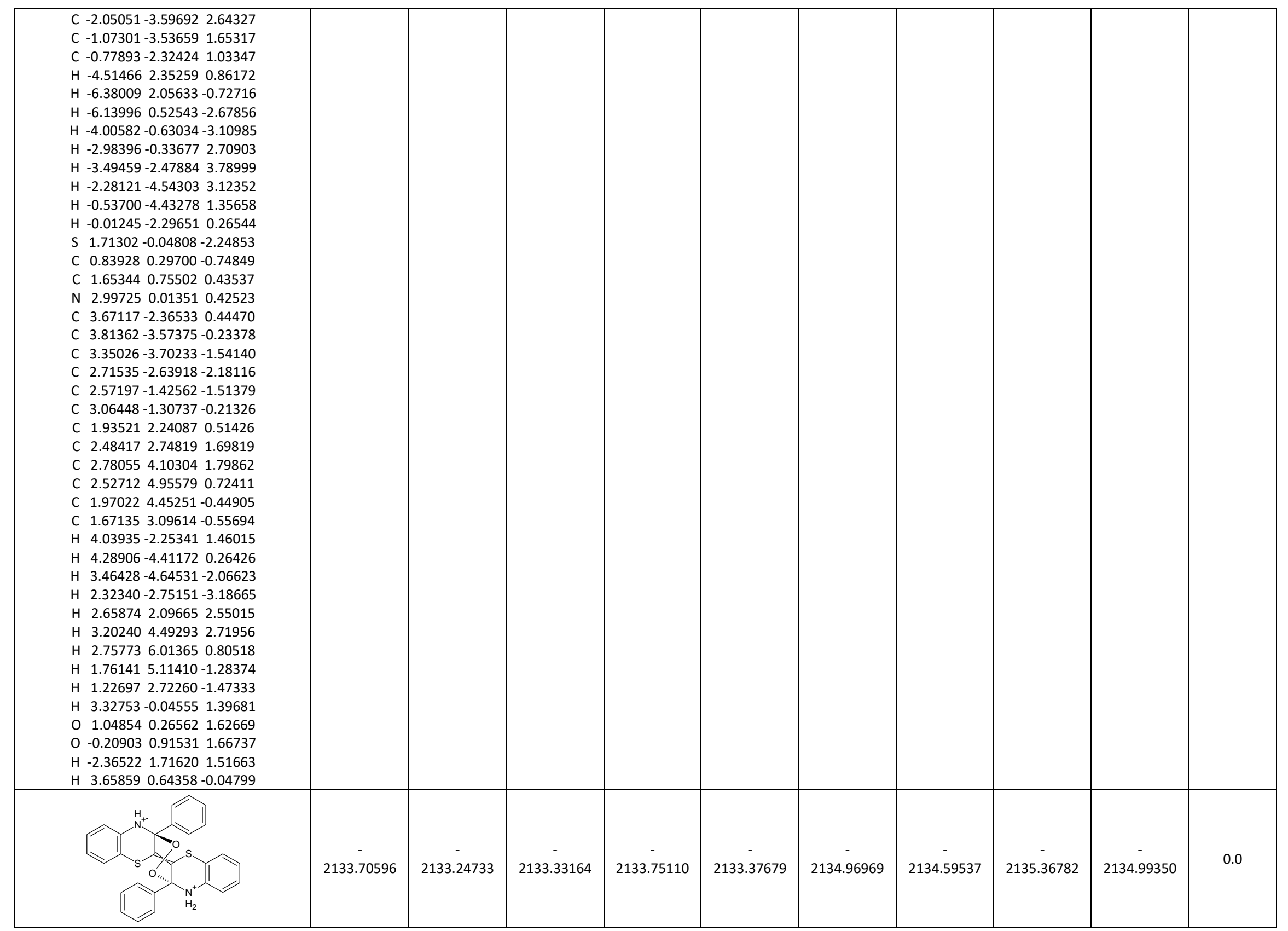

S175 
S $-1.77636-1.31075-1.45934$ C $-0.75023-0.46843-0.32302$

C -1.46065 0.479680 .61363

N $-2.36345-0.334111 .42249$

C $-4.27825-1.731711 .73121$

C - $5.24606-2.526081 .17947$

C $-5.21306-2.85652-0.19954$

C $-4.18308-2.41630-1.00519$

C $-3.15713-1.62974-0.46135$

C $-3.23586-1.217120 .91353$

C - $-2.168821 .63243-0.08633$

C $-1.531192 .26015-1.16108$

C $-2.131293 .34835-1.78451$

C $-3.367613 .81516-1.33735$

C $-3.999353 .19394-0.26260$

$\begin{array}{llll}C & -3.40256 & 2.10287 & 0.36619\end{array}$

H - $-4.31471-1.441112 .77619$

H -6.05698-2.89366 1.79899

- $-5.99228-3.48238-0.62082$

H $-4.13777-2.71078-2.04878$

H $-0.568401 .90174-1.51370$

H - $-1.634693 .82876-2.62175$

H -3.83735 $4.66245-1.82777$

H -4.959933 .555390 .09078$

H -3.90932 1.632201 .20228

S $1.37755-1.86365-1.27001$

C $0.57789-0.70347-0.23869$

C 1.326330 .002740 .87871

N 2.26269-1.01306 1.51607

C $4.65825-1.421461 .10035$

C $5.67711-1.834550 .24635$

C $5.37734-2.22849-1.05605$

C $4.06587-2.20743-1.52158$

C $3.03582-1.80650-0.66957$

C $3.35458-1.427270 .63152$

C 2.085801 .254140 .50942

C 2.501172 .106531 .53912

C 3.223603 .254911 .23533

C $3.529893 .55880-0.09150$

C $3.109432 .71407-1.11584$

C $2.386731 .56091-0.81852$

H $4.87920-1.106392 .11611$

H $6.70190-1.840450 .60078$

H $6.17174-2.54392-1.72434$

H $3.84049-2.49692-2.54299$

H 2.242641 .891892 .57250

H 3.539903 .916692 .03536

H $4.091464 .45801-0.32585$

H $3.339482 .95010-2.14992$

H $2.062390 .91395-1.62822$

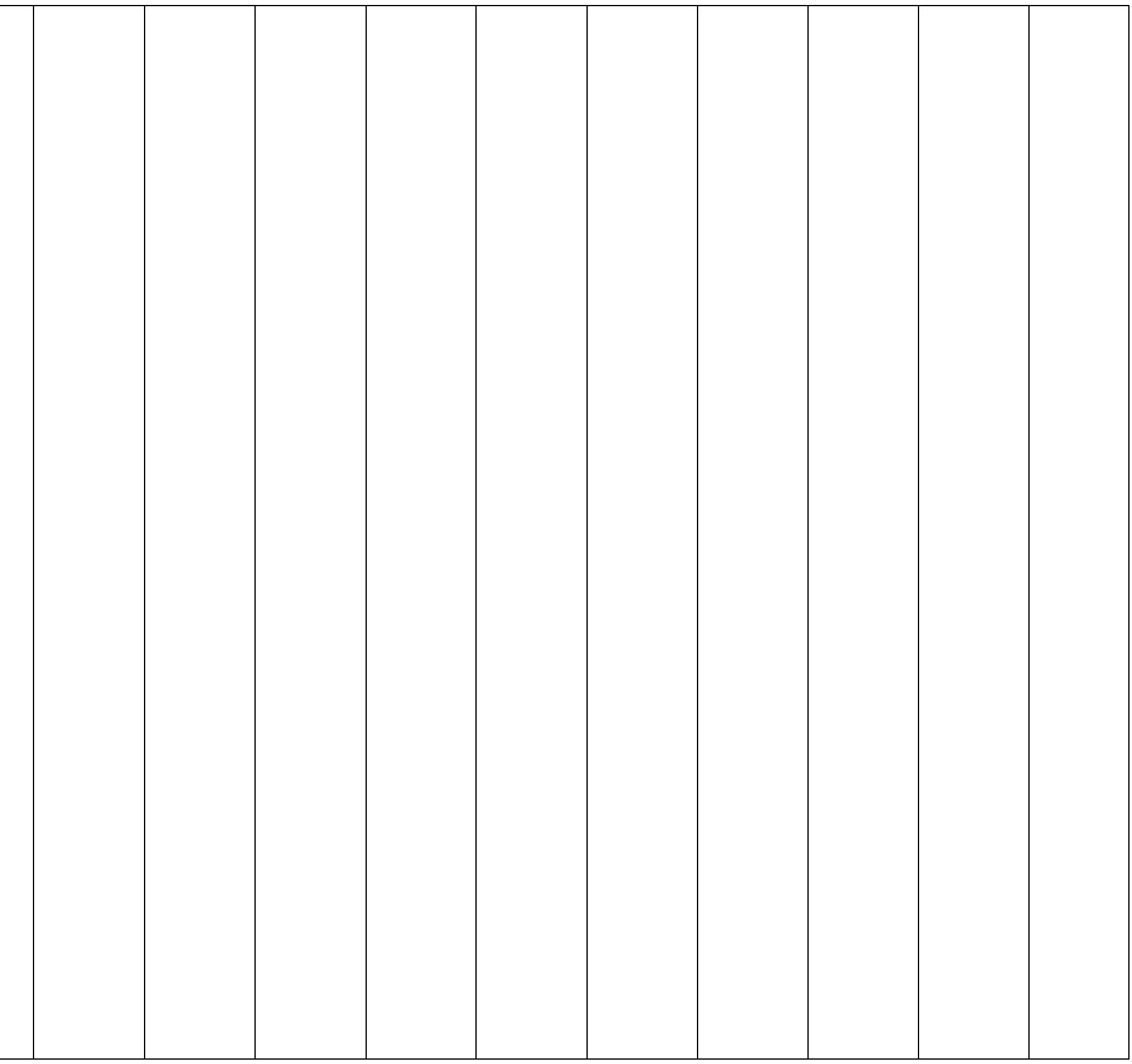




\begin{tabular}{|c|c|c|c|c|c|c|c|c|c|c|}
\hline $\begin{array}{rrrr}H & 2.65266 & -0.58710 & 2.36540 \\
\mathrm{O} & 0.42324 & 0.19911 & 1.95488 \\
\mathrm{O} & -0.54954 & 1.13380 & 1.47470 \\
\mathrm{H} & -2.43350 & -0.08414 & 2.40496 \\
\mathrm{H} & 1.71178 & -1.82461 & 1.83129 \\
\end{array}$ & & & & & & & & & & \\
\hline $\begin{array}{c}\text { Single-bond dimer, C2-hydroperoxy } \\
\text { radical }\end{array}$ & & & & & & & & & & \\
\hline \begin{tabular}{lrrr}
\multicolumn{4}{c}{ H } \\
S & -1.15520 & -2.09766 & -0.59066 \\
C & -0.48420 & -0.88824 & 0.60781 \\
C & -1.33645 & 0.35888 & 0.80659 \\
N & -2.62769 & 0.30117 & 0.60328 \\
C & -4.82230 & -0.50169 & 0.07815 \\
C & -5.65635 & -1.46835 & -0.45789 \\
C & -5.11010 & -2.63088 & -1.00950 \\
C & -3.73488 & -2.83199 & -1.02158 \\
C & -2.88605 & -1.86329 & -0.48243 \\
C & -3.43678 & -0.69886 & 0.05844 \\
C & -0.79537 & 1.57527 & 1.40375 \\
C & 0.29489 & 1.53046 & 2.29180 \\
C & 0.75205 & 2.69919 & 2.88569 \\
C & 0.13985 & 3.92007 & 2.60243 \\
C & -0.93683 & 3.97426 & 1.71652 \\
C & -1.40280 & 2.81321 & 1.11840 \\
H & -5.23290 & 0.40479 & 0.51434 \\
H & -6.73037 & -1.31923 & -0.44542 \\
H & -5.76114 & -3.39089 & -1.42906 \\
H & -3.31724 & -3.74030 & -1.44457 \\
H & 0.75138 & 0.58549 & 2.56038 \\
H & 1.58211 & 2.65324 & 3.58316 \\
H & 0.50435 & 4.83028 & 3.06854 \\
H & -1.40174 & 4.92476 & 1.47626 \\
H & -2.19725 & 2.88536 & 0.37988 \\
C & 0.95589 & -0.67133 & 0.15558
\end{tabular} & $\begin{array}{c}- \\
- \\
2133.72927\end{array}$ & $\begin{array}{c}- \\
2133.27153\end{array}$ & $\begin{array}{c}- \\
2133.35890\end{array}$ & 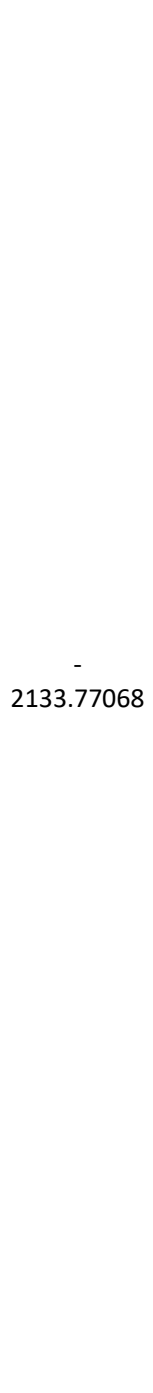 & $\begin{array}{l}- \\
- \\
- \\
2133.40031\end{array}$ & 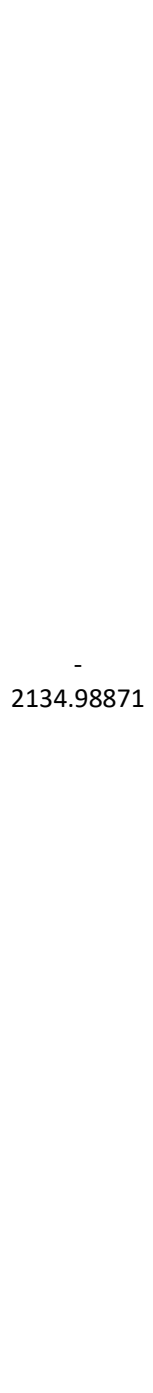 & $\begin{array}{c}- \\
2134.61834\end{array}$ & $\begin{array}{c}- \\
2135.38753\end{array}$ & $\begin{array}{c}- \\
2135.01716\end{array}$ & 0.0 \\
\hline
\end{tabular}




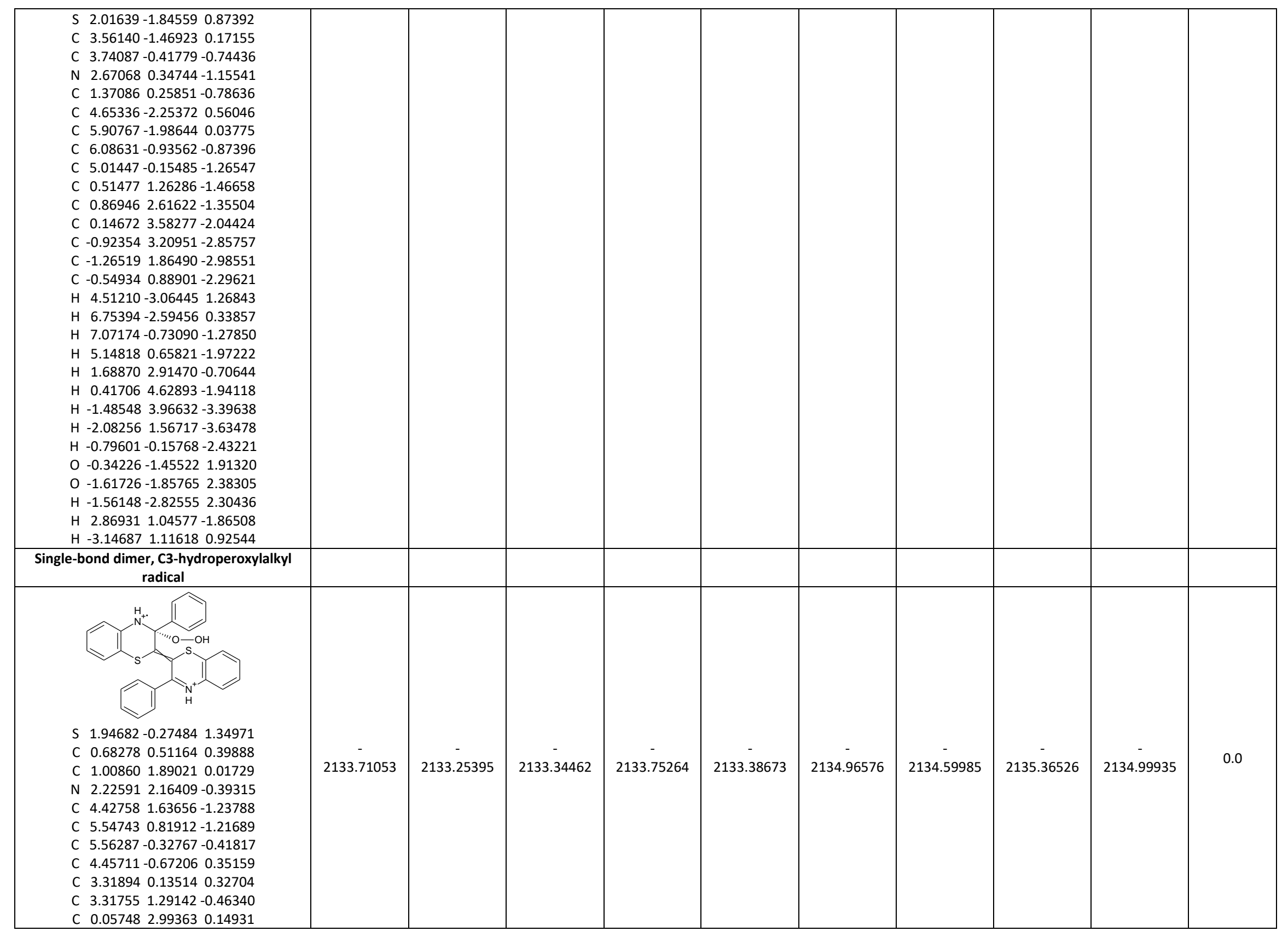




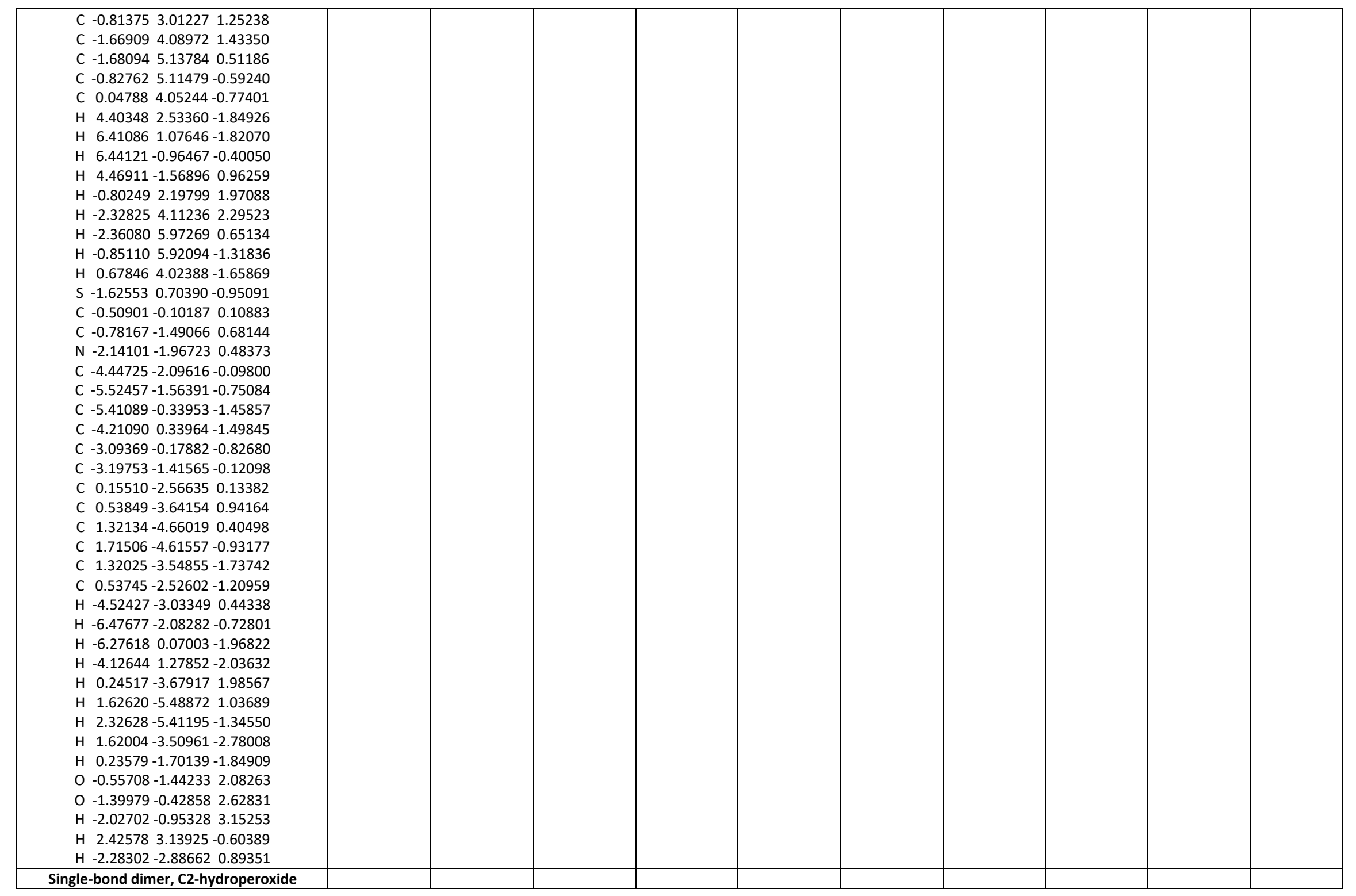




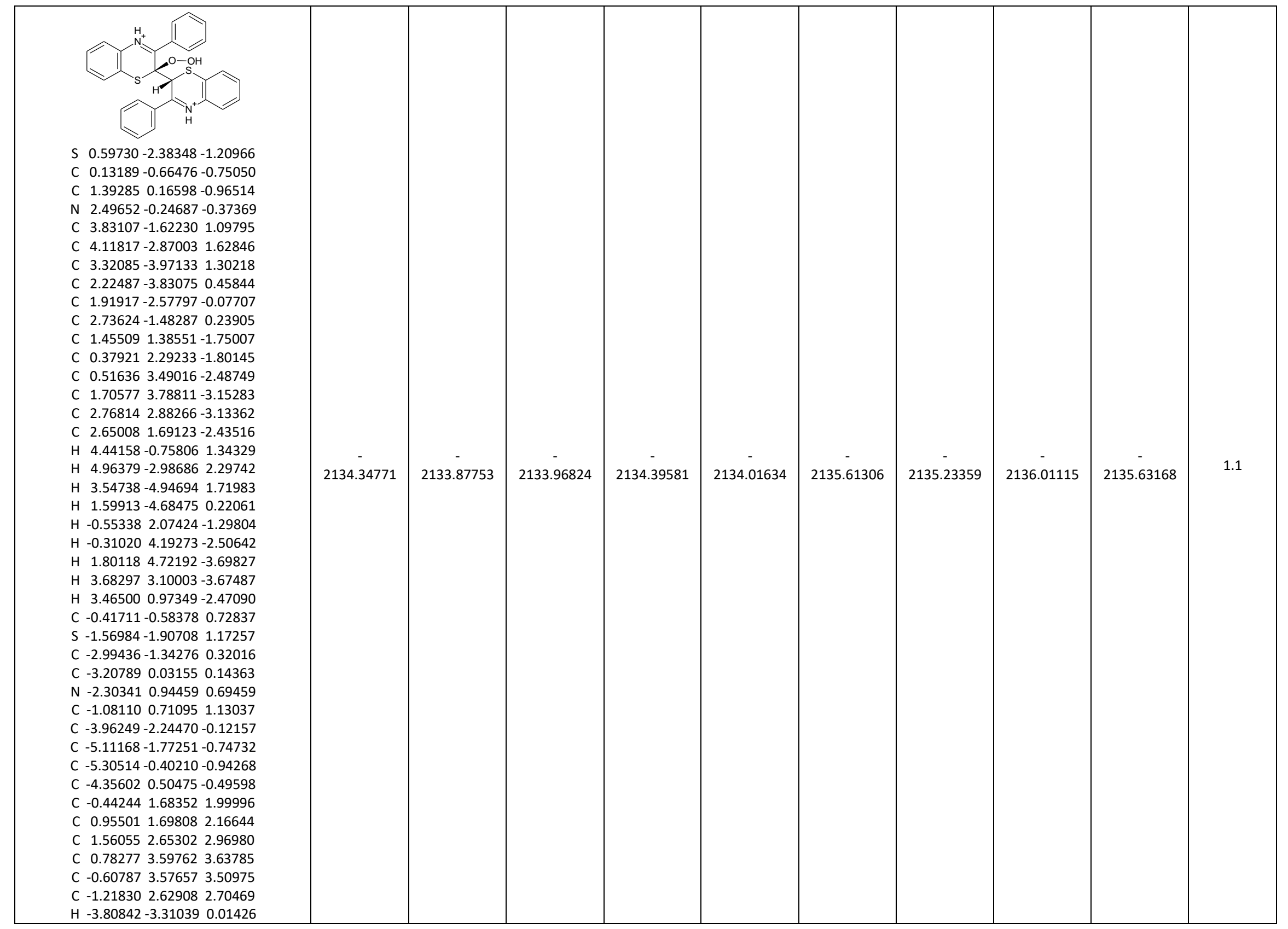




\begin{tabular}{|c|c|c|c|c|c|c|c|c|c|c|}
\hline $\begin{array}{lrrr}H & -5.85667 & -2.48063 & -1.09541 \\
H & -6.19842 & -0.04186 & -1.44142 \\
H & -4.49688 & 1.57237 & -0.63697 \\
H & 1.58534 & 0.97611 & 1.66280 \\
H & 2.63991 & 2.65670 & 3.07742 \\
H & 1.25689 & 4.33900 & 4.27334 \\
H & -1.21772 & 4.28897 & 4.05517 \\
H & -2.30329 & 2.60143 & 2.67691 \\
O & -0.93100 & -0.26081 & -1.55154 \\
O & -0.59405 & -0.43003 & -2.92973 \\
H & -1.33402 & -0.98619 & -3.22697 \\
H & 0.43019 & -0.75502 & 1.39572 \\
H & 3.29464 & 0.38306 & -0.42046 \\
H & -2.68205 & 1.87059 & 0.87290 \\
\end{array}$ & & & & & & & & & & \\
\hline $\begin{array}{lrlr}\text { S } & 1.21893 & -1.03079 & 1.58143 \\
C & -0.16606 & -0.41619 & 0.52683 \\
C & -0.88599 & -1.67355 & 0.04158 \\
\mathrm{~N} & -0.15254 & -2.56777 & -0.58715 \\
\mathrm{C} & 1.85176 & -3.47996 & -1.58220 \\
\mathrm{C} & 3.22402 & -3.66889 & -1.54055 \\
\mathrm{C} & 3.98682 & -3.04947 & -0.54618 \\
\mathrm{C} & 3.38756 & -2.22248 & 0.39679 \\
\mathrm{C} & 2.00688 & -2.01350 & 0.36090 \\
\mathrm{C} & 1.24531 & -2.66129 & -0.62460 \\
\mathrm{C} & -2.29129 & -1.96531 & 0.25993 \\
\mathrm{C} & -3.27780 & -0.96159 & 0.24085 \\
\mathrm{C} & -4.61308 & -1.30259 & 0.39666 \\
\mathrm{C} & -4.98257 & -2.63334 & 0.59657 \\
\mathrm{C} & -4.00977 & -3.63306 & 0.64487 \\
\mathrm{C} & -2.67298 & -3.30630 & 0.47632 \\
\mathrm{H} & 1.24621 & -3.96153 & -2.34405 \\
\mathrm{H} & 3.70113 & -4.30176 & -2.28091 \\
\mathrm{H} & 5.06088 & -3.20079 & -0.51344 \\
\mathrm{H} & 3.98588 & -1.72847 & 1.15536 \\
\mathrm{H} & -3.00568 & 0.07370 & 0.08471 \\
\mathrm{H} & -5.36990 & -0.52563 & 0.36318 \\
\mathrm{H} & -6.02921 & -2.89040 & 0.72800 \\
\mathrm{H} & -4.29185 & -4.66435 & 0.82950 \\
\mathrm{H} & -1.92143 & -4.08479 & 0.57288 \\
\mathrm{C} & 0.32770 & 0.45904 & -0.67242 \\
\mathrm{~S} & -0.92083 & 0.78264 & -1.95127 \\
\mathrm{C} & -1.79814 & 2.09197 & -1.17180 \\
\end{array}$ & $\stackrel{-}{-}$ & 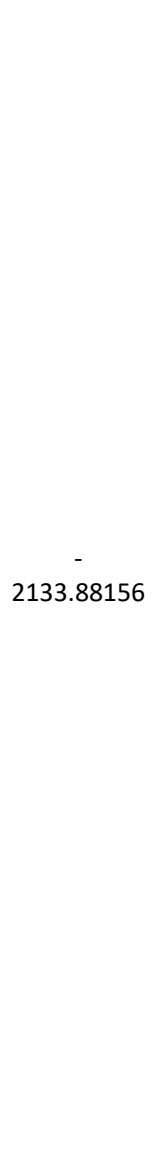 & $\begin{array}{c}- \\
2133.96934\end{array}$ & $\begin{array}{c}- \\
2134.39741\end{array}$ & $\begin{array}{c}- \\
- \\
2134.01483\end{array}$ & $\begin{array}{c}- \\
- \\
2135.61800\end{array}$ & $\begin{array}{c}- \\
2135.23542\end{array}$ & $\begin{array}{c}- \\
2136.01606\end{array}$ & $\begin{array}{c}- \\
- \\
2135.63348\end{array}$ & 0.0 \\
\hline
\end{tabular}




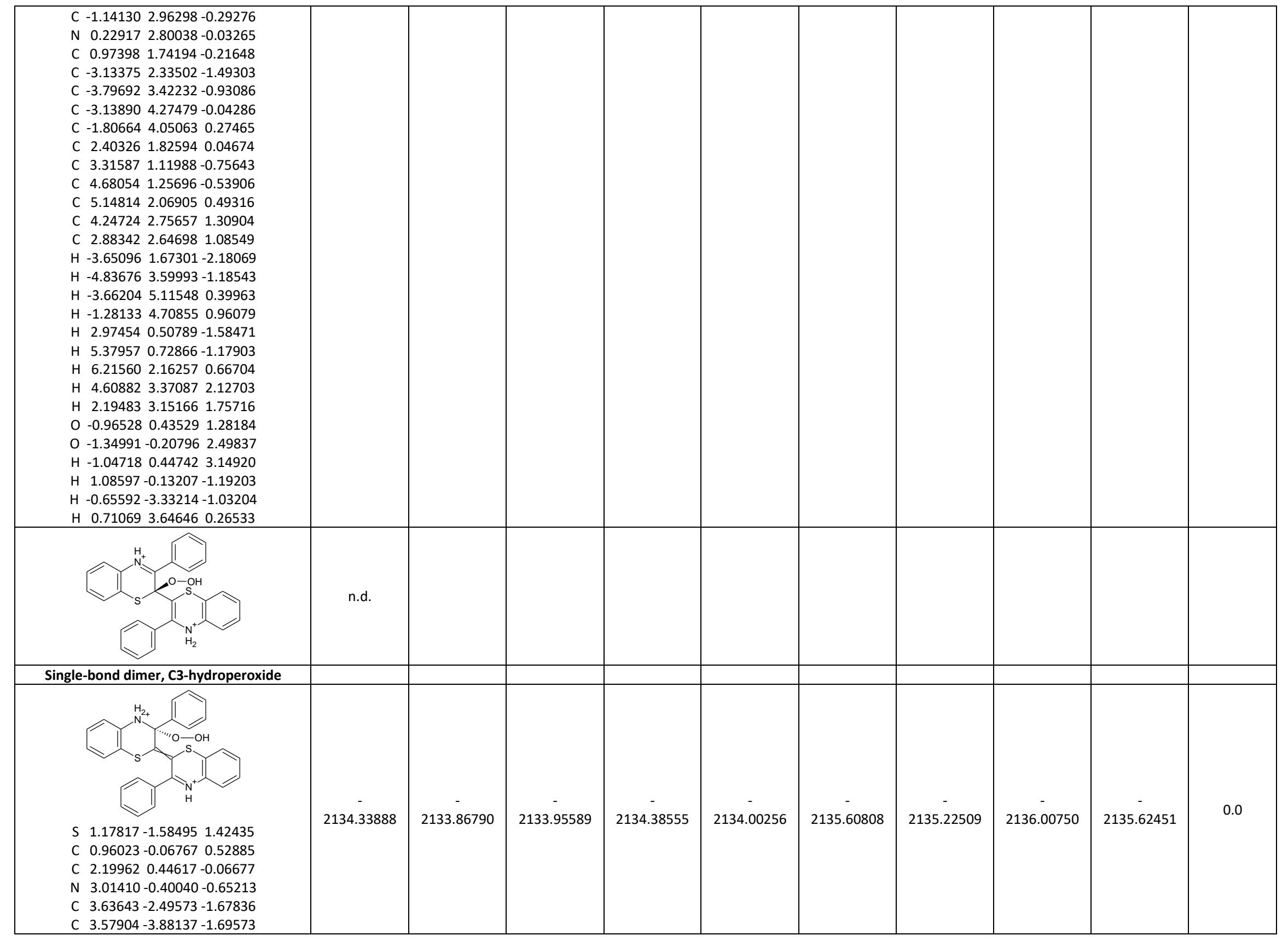


C $2.77933-4.55948-0.77258$

C $2.02514-3.858610 .16285$

C $2.06763-2.464070 .18418$

C $2.88110-1.79246-0.73723$

$\begin{array}{lllll}C & 2.58513 & 1.84976 & 0.01374\end{array}$

$\begin{array}{llll}C & 2.29073 & 2.57087 & 1.18502\end{array}$

C 2.712353 .888451 .30563

C 3.402414 .500930 .25969

$3.683813 .79260-0.91089$

C $3.288472 .46892-1.03539$

H $4.25772-1.95591-2.38670$

H $4.15903-4.43349-2.42725$

H $2.73633-5.64375-0.78465$

H $1.39938-4.389380 .87333$

H 1.763432 .090502 .00428

H 2.501234 .438172 .21708

H 3.718065 .535500 .35290

H $4.204514 .27698-1.73044$

H $3.477651 .93990-1.96570$

S $-0.418552 .13292-0.36926$

$\begin{array}{llll}C & -0.23507 & 0.57210 & 0.44892\end{array}$

C $-1.44375-0.037821 .16809$

N -2.57899 1.005791 .20325

C $-4.373351 .28695-0.48649$

C $-4.74043 \quad 1.72766-1.75647$

C $-3.787892 .27806-2.61287$

C $-2.45408 \quad 2.38666-2.22330$

C - $2.082031 .95688-0.95149$

C $-3.049551 .42913-0.10502$

C $-1.97765-1.316970 .56127$

C $-2.75258-2.176581 .34857$

C $-3.29438-3.326810 .78278$

C $-3.07573-3.61686-0.56330$

C - $2.31164-2.75450-1.34707$

C $-1.76068-1.60478-0.78846$

H -5.105630 .852990 .18766$

H -5.77309 $1.63518-2.07510$

H $-4.080662 .61506-3.60201$

H -1.71010 $2.79580-2.89888$

H -2.92125 -1.96563 2.40009

H -3.88645 -3.99714 1.39783

H $-3.50088-4.51502-1.00092$

H -2.13979-2.97474-2.39590

H $-1.15999-0.94467-1.40689$

H -3.34312 0.594391 .74874

O $-1.15656-0.296802 .50929$

O $-\begin{array}{lllll}0.70547 & 0.94402 & 3.08297\end{array}$

H $-1.072280 .86383 \quad 3.97973$

H $3.87887-0.01571-1.02458$

H -2.222261 .805651 .75092$

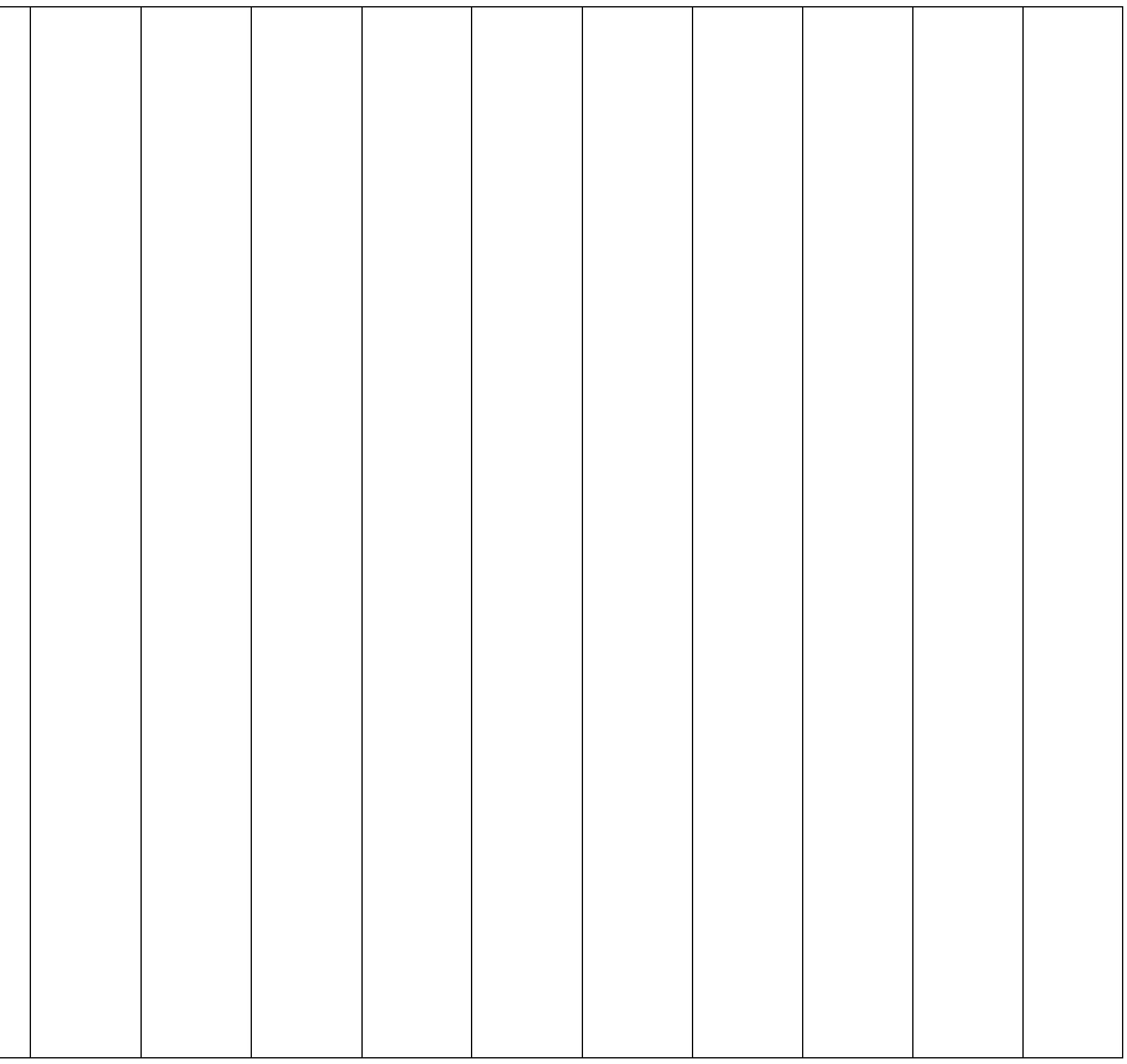




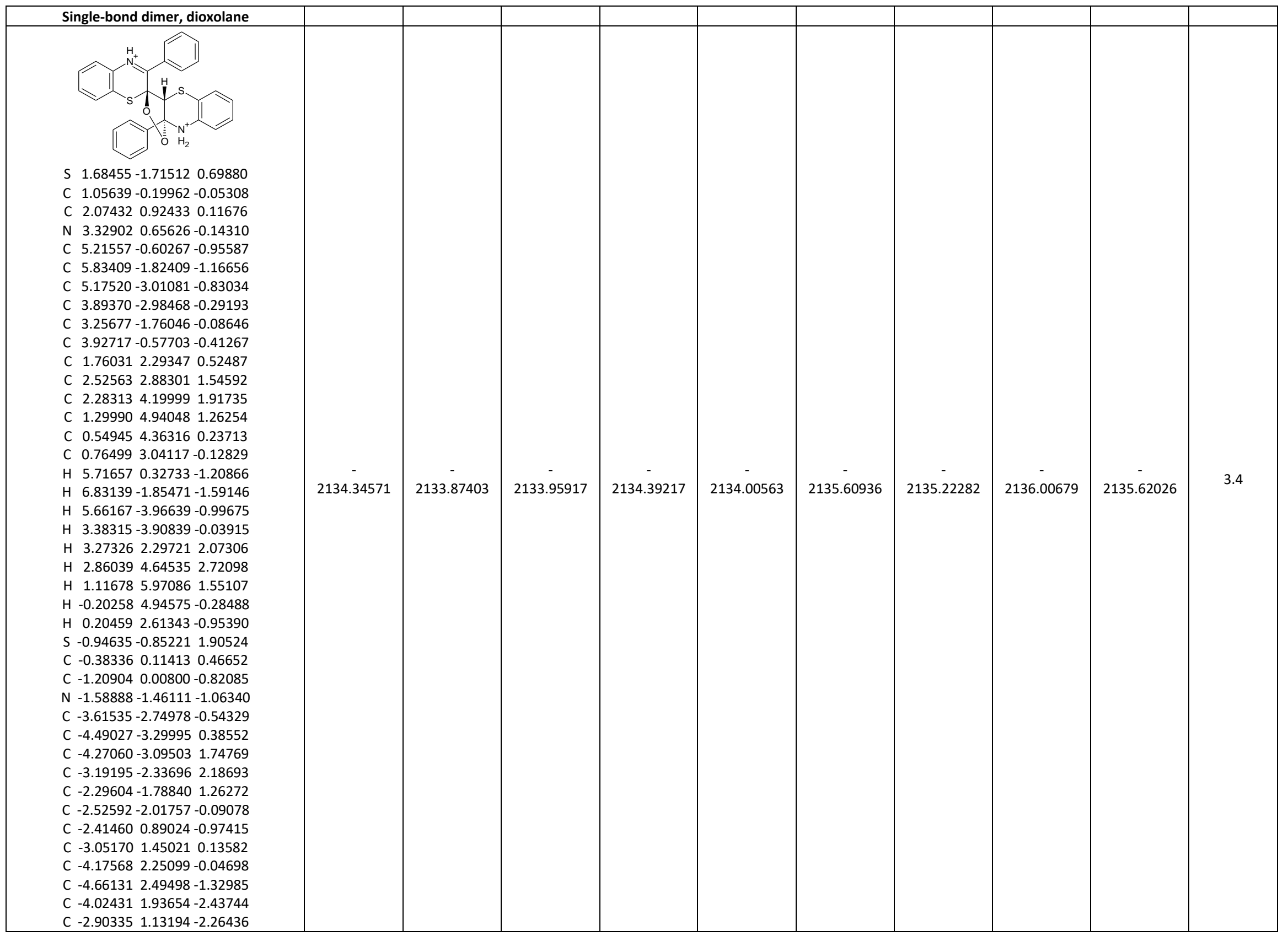




\begin{tabular}{|c|c|c|c|c|c|c|c|c|c|c|}
\hline $\begin{array}{llll}H & -0.42027 & 1.15727 & 0.78510 \\
H & -3.77326 & -2.89504 & -1.60823 \\
H & -5.34132 & -3.87933 & 0.04505 \\
H & -4.95372 & -3.51640 & 2.47804 \\
H & -3.04386 & -2.16134 & 3.24796 \\
H & -2.68975 & 1.26626 & 1.14321 \\
H & -4.66989 & 2.68336 & 0.81704 \\
H & -5.53714 & 3.12165 & -1.46752 \\
H & -4.39645 & 2.12882 & -3.43857 \\
H & -2.40591 & 0.71223 & -3.13431 \\
O & 0.87502 & -0.44906 & -1.45929 \\
O & -0.28396 & 0.32988 & -1.82235 \\
\text { H } & -1.99538 & -1.51857 & -2.00356 \\
H & -0.71862 & -2.01328 & -1.10319 \\
\text { H } & 3.96087 & 1.45520 & -0.13861 \\
\end{array}$ & & & & & & & & & & \\
\hline $\begin{array}{llll} & \end{array}$ & $\begin{array}{c}- \\
2134.32683\end{array}$ & $\begin{array}{c}2 \\
- \\
- \\
2133.85473\end{array}$ & $\begin{array}{c}- \\
2133.94015\end{array}$ & 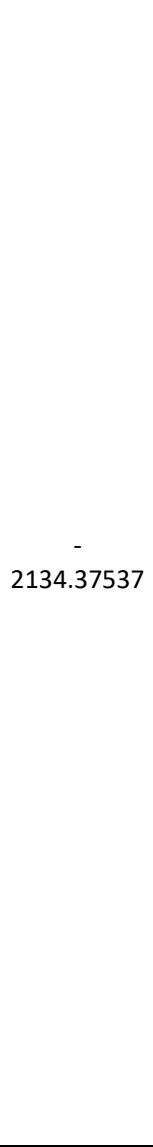 & $\begin{array}{c}- \\
2133.98869\end{array}$ & $\begin{array}{l}- \\
2135.59397\end{array}$ & $\begin{array}{c}- \\
- \\
\\
\\
\\
\\
\end{array}$ & $\begin{array}{c}- \\
2135.99130\end{array}$ & $\begin{array}{c}- \\
\\
\\
\\
\\
\\
\end{array}$ & 13.2 \\
\hline
\end{tabular}




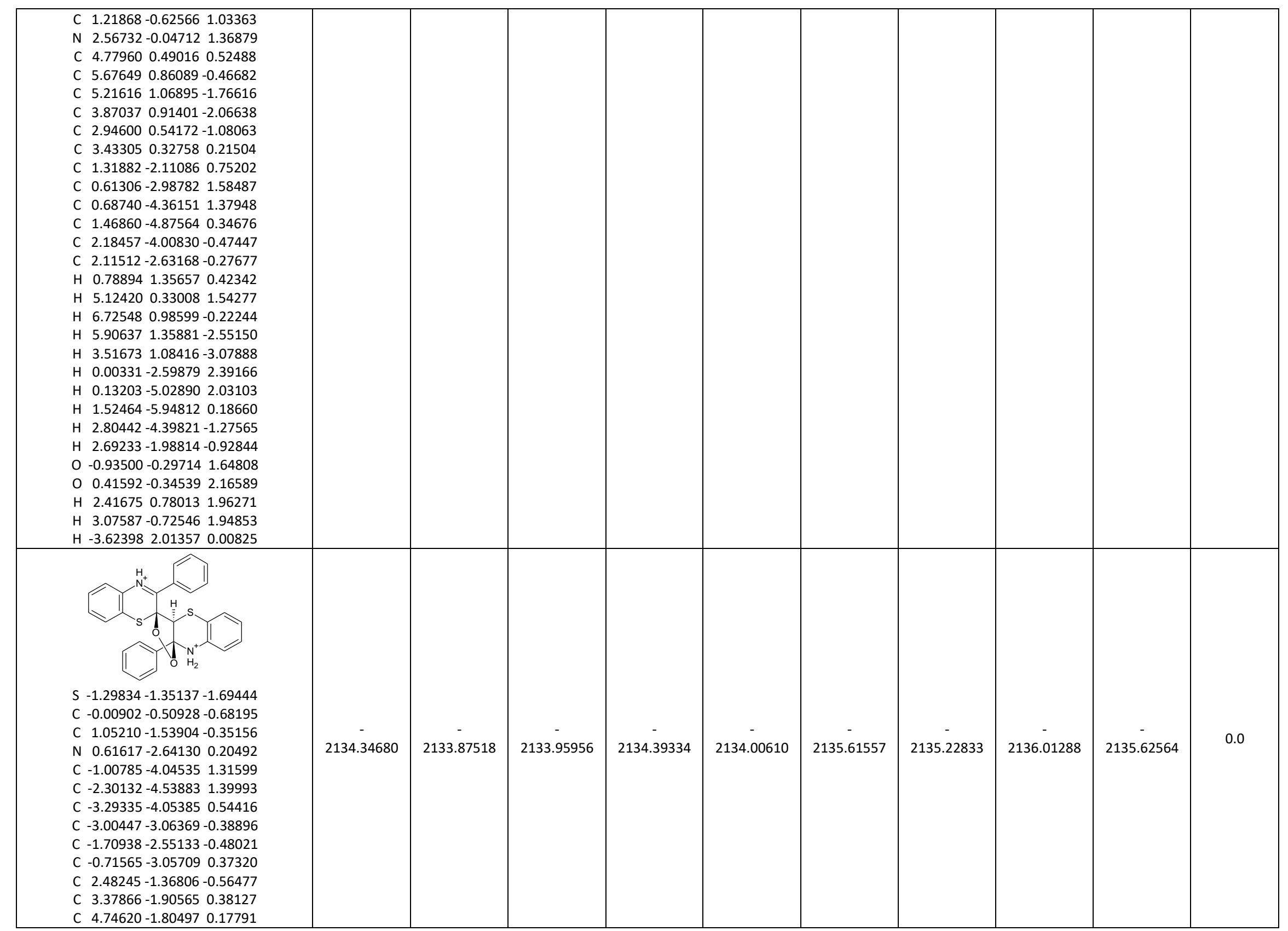




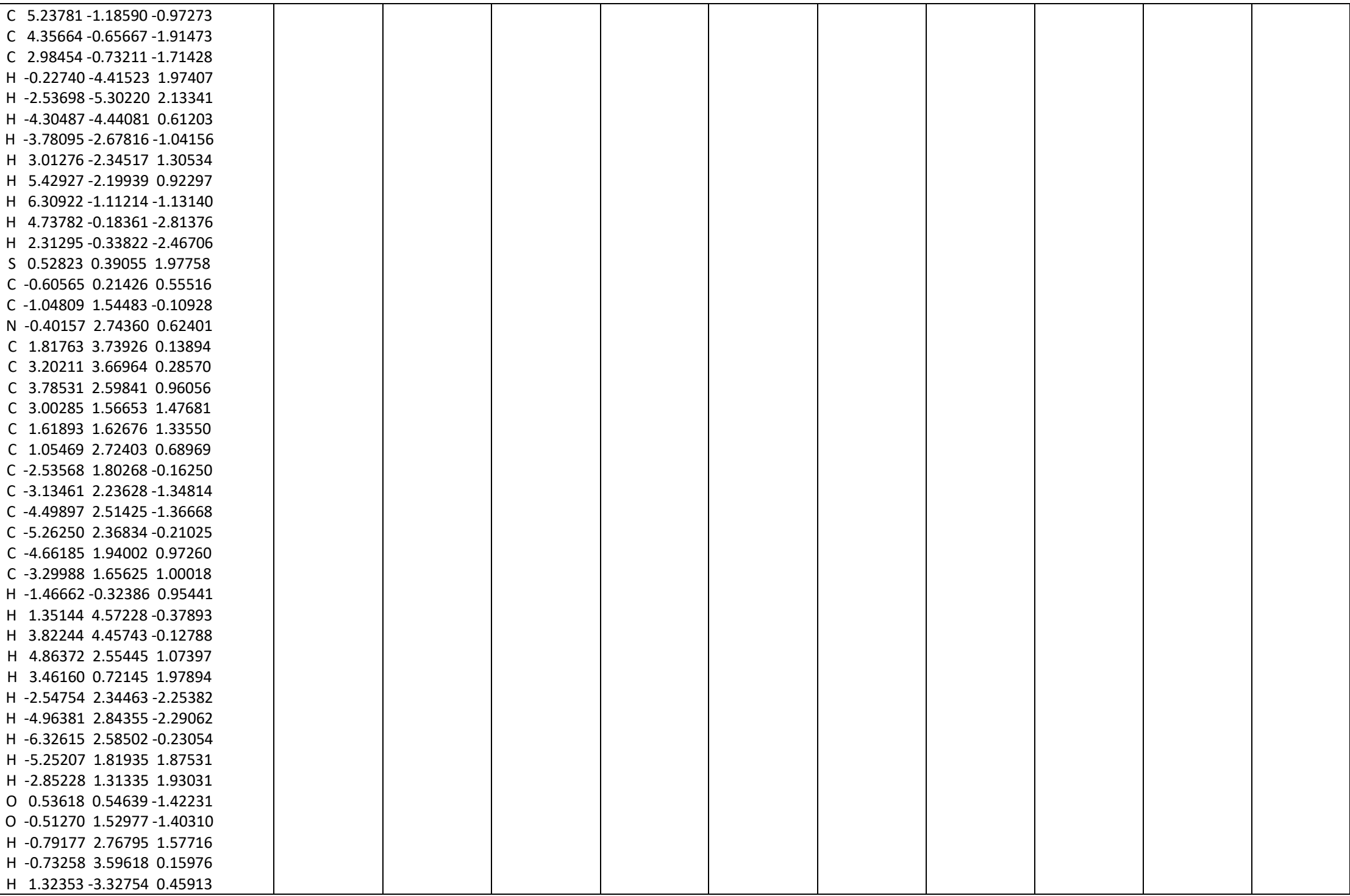




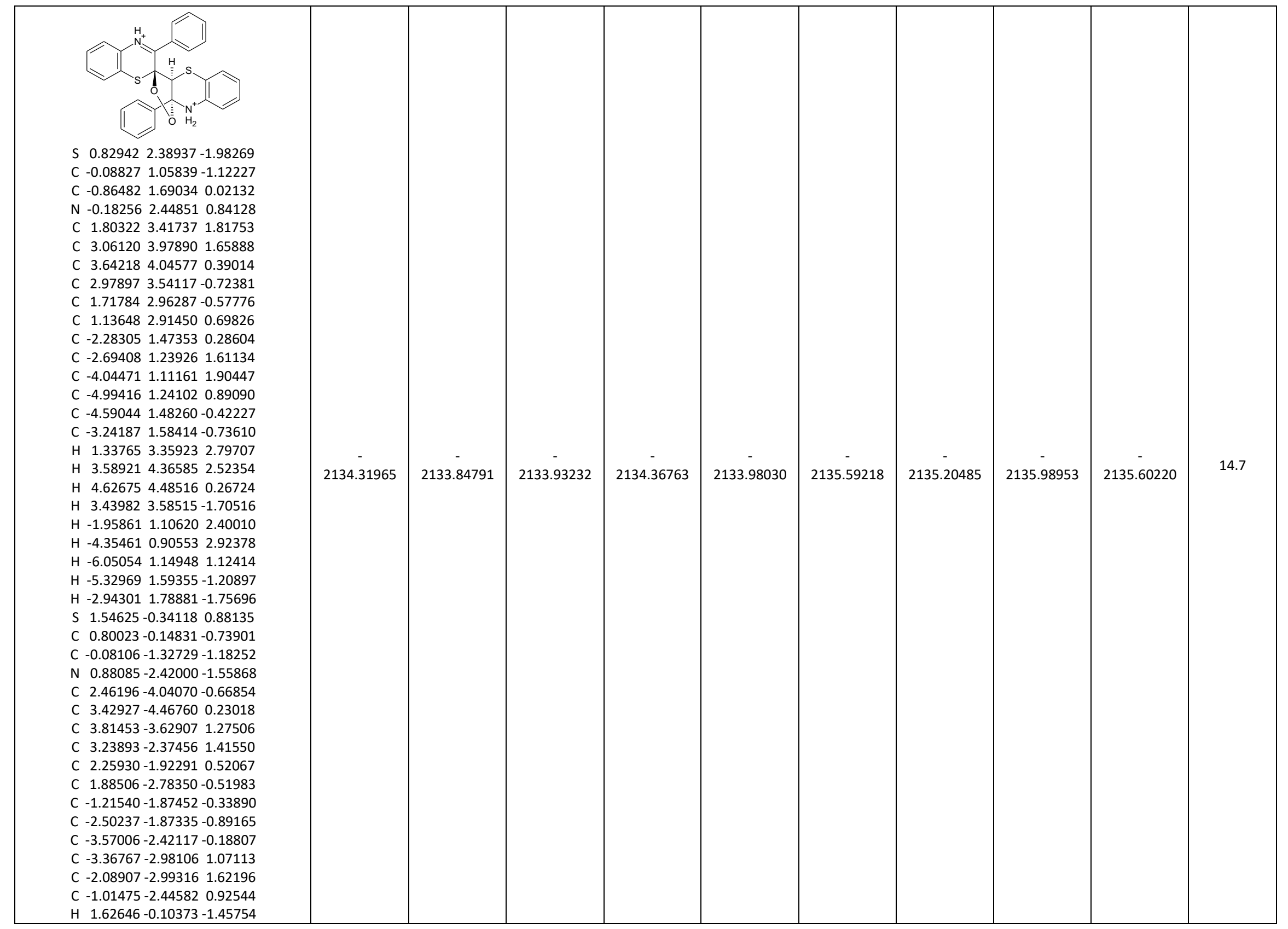




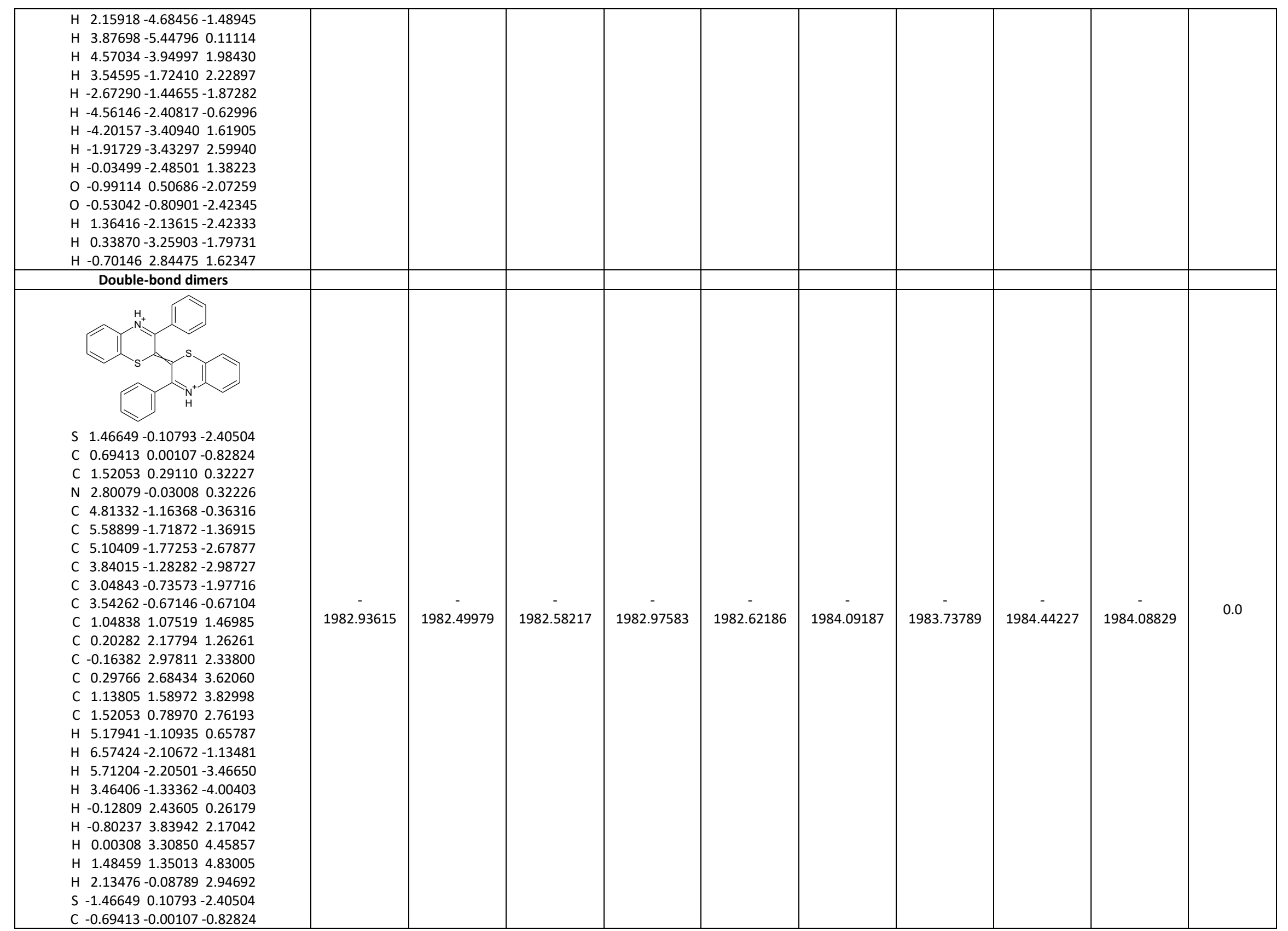




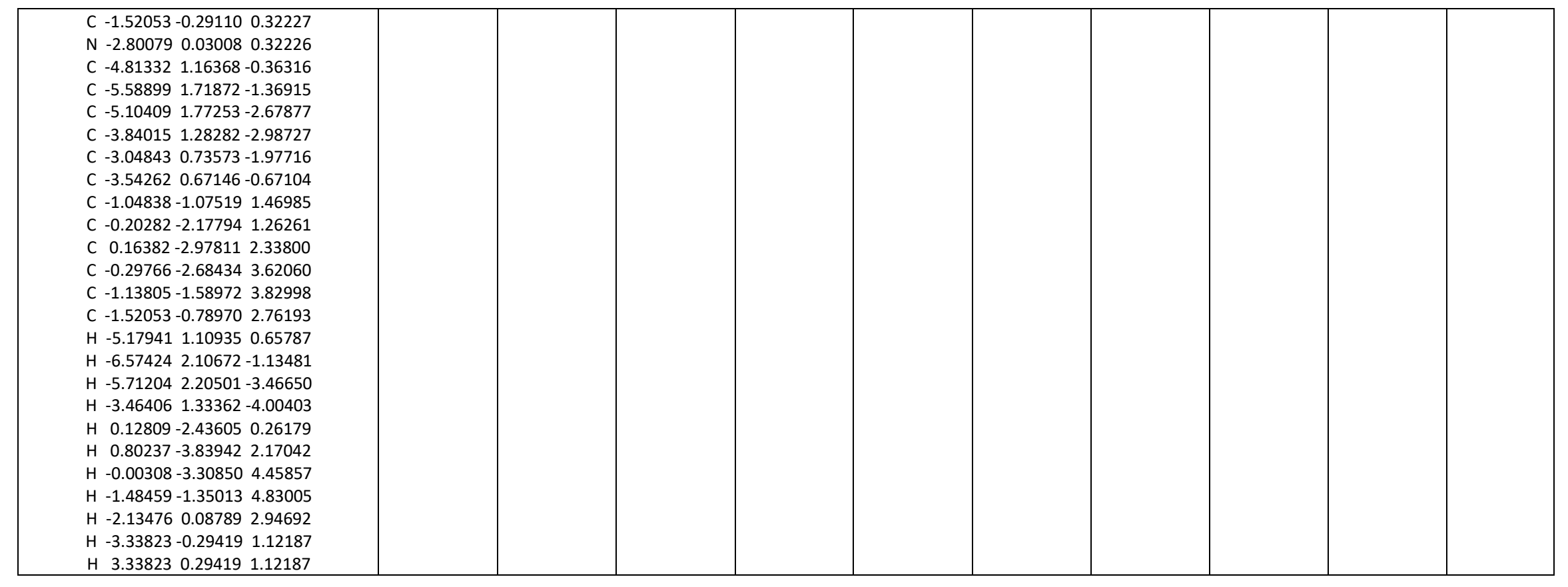


Table S7. $\mathrm{p} K_{\mathrm{a}}$ values computed in methanol at different theory levels for all bibenzothiazine derivatives examined. For each chemical species and for each protonation state, the $G_{R R H O}$,calc value (see Tables S4-S6) of the most stable stereoisomer identified was used in the calculation.

\begin{tabular}{|c|c|c|c|c|c|c|}
\hline & \multicolumn{2}{|c|}{ PBE0 / 6-31+G(d,p) / SMD } & \multicolumn{2}{|c|}{ M062X / 6-31+G(d,p) / SMD } & \multicolumn{2}{|c|}{ M062X / 6-311++G(2d,2p) / SMD } \\
\hline & $\mathrm{p} K_{\mathrm{a} 1}$ & $\mathrm{p} K_{\mathrm{a} 2}$ & $\mathrm{p} K_{\mathrm{a} 1}$ & $\mathrm{p} K_{\mathrm{a} 2}$ & $\mathrm{p} K_{\mathrm{a} 1}$ & $\mathrm{p} K_{\mathrm{a} 2}$ \\
\hline Single-bond dimer & 4.3 & 3.2 & 3.6 & 2.3 & 3.6 & 2.4 \\
\hline Single-bond dimer, radical & 6.6 & 1.6 & 6.6 & 0.3 & 6.6 & 0.4 \\
\hline Single-bond dimer, C2-peroxyl radical & 3.7 & 1.9 & 3.0 & 1.2 & 3.0 & 1.3 \\
\hline Single-bond dimer, C3-peroxyl radical & 4.2 & -2.0 & 3.4 & -2.4 & 3.5 & -2.3 \\
\hline Single-bond dimer, dioxolane radical & 4.9 & 2.0 & 4.8 & 0.7 & 4.9 & 0.8 \\
\hline Single-bond dimer, dioxane radical & 4.7 & -1.0 & 4.2 & -1.1 & 4.4 & -1.2 \\
\hline Single-bond dimer, C2-hydroperoxylalkyl radical & 5.4 & 2.4 & 5.4 & 1.5 & 5.6 & 1.6 \\
\hline Single-bond dimer, C3-hydroperoxylalkyl radical & 5.0 & 2.7 & 4.8 & 2.1 & 5.2 & 1.9 \\
\hline Single-bond dimer, C2-hydroperoxide & 3.8 & 3.1 & 3.5 & 2.2 & 3.5 & 2.2 \\
\hline Single-bond dimer, C3-hydroperoxide & 4.3 & 0.6 & 3.7 & 0.3 & 3.8 & 0.3 \\
\hline Single-bond dimer, dioxolane & 3.1 & -0.4 & 2.7 & -0.9 & 2.8 & -0.7 \\
\hline Double-bond dimers & 4.6 & 2.4 & 4.2 & 1.3 & 4.3 & 1.4 \\
\hline
\end{tabular}


Table S8. $\mathrm{p} K_{\mathrm{a}}$ values computed in methanol at different theory levels for some reference nitrogen bases and corresponding experimental values. For each chemical species and for each protonation state, the $G_{R R H O}$,calc value of the most stable stereoisomer identified was used in the calculation. At each theory level, a linear fitting with respect to the experimental values was used to correct the raw $\mathrm{p} K_{\mathrm{a}}$ values.

\begin{tabular}{|c|c|c|c|c|}
\hline & PBE0 / 6-31+G(d,p) / SMD & M062X / 6-31+G(d,p) / SMD & M062X / 6-311++G(2d,2p) / SMD & Experimental $^{a}$ \\
\hline pyridine & 5.87 & 5.90 & 5.85 & 5.44 \\
\hline aniline & 4.23 & 4.42 & 4.30 & 6.05 \\
\hline 2-chloroaniline & 2.95 & 2.88 & 2.81 & 3.71 \\
\hline 4-chloroaniline & 3.77 & 3.87 & 3.76 & 4.95 \\
\hline 2-nitroaniline & 0.73 & 0.39 & 0.51 & 0.20 \\
\hline 4-nitroaniline & 1.30 & 1.46 & 1.46 & 1.55 \\
\hline 4-chloro-2-nitroaniline & 0.69 & 0.73 & 0.86 & -0.67 \\
\hline 2-methylpyridine & 6.39 & 6.42 & 6.43 & 6.18 \\
\hline quinoline & 5.84 & 5.73 & 5.74 & 5.16 \\
\hline 2-methylquinoline & 6.86 & 6.83 & 6.90 & 6.06 \\
\hline
\end{tabular}

[a] Rived, F.; Rosés, M.; Bosch, E. Anal. Chim. Acta 1998, 374, 309-324. 